NPL影

National Physical Laboratory

NFL REPORT IR 59

ENVIRONMENTAL RADIOACTIVITY PROFICIENCY TEST EXERCISE 2020

VAN ES, E M; COLLINS, S M; MOHAMUD, H AND SHEARMAN, R

NOVEMBER 2021

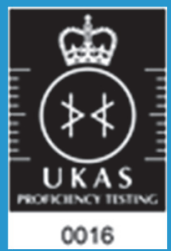





\title{
Environmental Radioactivity Proficiency Test Exercise 2020
}

\author{
Van Es, E M+' Collins, S M; Mohamud, $\mathrm{H}$ and \\ Shearman, $\mathrm{R}$ \\ Medical, Marine and Nuclear Department
}

\begin{abstract}
The results of NPL's twenty-sixth Environmental Radioactivity Proficiency Test Exercise are reported. Five different sample types were offered: an aqueous mixture of one alpha emitting radionuclide and two beta emitting radionuclides (designated ' $A B$ '), an aqueous mixture of three alpha-emitting radionuclides ('A1'), an aqueous mixture of three beta-emitting radionuclides ('B1'), an aqueous mixture of three gamma-emitting radionuclides ('GH'), and a second aqueous mixture of five gamma-emitting radionuclides ('GL'). In total, 392 results were submitted. A total of 377 were submitted for individual radionuclide analysis, of these results 299 (79 \%) were in agreement with the proficiency test exercise assigned values, $46(12 \%)$ were questionable and $32(8 \%)$ were discrepant.
\end{abstract}


NPL REPORT IR 59

(C) NPL Management Limited, 2021

ISSN 1754-2952

https://doi.org/10.47120/npl.IR59

National Physical Laboratory

Hampton Road, Teddington, Middlesex, TW11 OLW

Extracts from this report may be reproduced provided the source is acknowledged and the extract is not taken out of context.

Approved on behalf of NPLML by Ben Russell,

Science Area Leader, Nuclear Metrology Group, Medical, Marine and Nuclear Department 
Assigned Values (reference time 2020-06-01 12:00:00 UTC)

\begin{tabular}{|c|c|}
\hline Radionuclide (AB) & Assigned Value $\left(\mathrm{Bq} \mathrm{g}^{-1}\right)$ \\
\hline${ }^{3} \mathrm{H}$ & $6.38 \pm 0.14$ \\
\hline${ }^{90} \mathrm{Sr}$ & $3.865 \pm 0.020$ \\
\hline${ }^{238} \mathrm{Pu}$ & $13.933 \pm 0.063$ \\
\hline Radionuclide (A1) & Assigned Value $\left(\mathrm{Bq} \mathrm{kg}^{-1}\right)$ \\
\hline $233 U$ & $2.0723 \pm 0.015$ \\
\hline${ }^{238} \mathrm{Pu}$ & $6.485 \pm 0.040$ \\
\hline${ }^{243} \mathrm{Am}$ & $4.476 \pm 0.090$ \\
\hline Radionuclide (B1) & Assigned Value $\left(\mathrm{Bq} \mathrm{g}^{-1}\right)$ \\
\hline${ }^{3} \mathrm{H}$ & $0.582 \pm 0.013$ \\
\hline${ }^{14} \mathrm{C}$ & $0.3253 \pm 0.0031$ \\
\hline 129 & $0.1941 \pm 0.0020$ \\
\hline Radionuclide (GH) & Assigned Value $\left(\mathrm{Bq} \mathrm{g}^{-1}\right)$ \\
\hline${ }^{60} \mathrm{Co}$ & $2.822 \pm 0.012$ \\
\hline${ }^{133} \mathrm{Ba}$ & $19.24 \pm 0.28$ \\
\hline${ }^{154} \mathrm{Eu}$ & $2.504 \pm 0.040$ \\
\hline Radionuclide (GL) & Assigned Value $\left(\mathrm{Bq} \mathrm{kg}^{-1}\right)$ \\
\hline${ }^{134} \mathrm{Cs}$ & $10.23 \pm 0.22$ \\
\hline${ }^{137} \mathrm{Cs}$ & $4.547 \pm 0.082$ \\
\hline${ }^{155} \mathrm{Eu}$ & $24.4 \pm 1.1$ \\
\hline${ }^{210} \mathrm{~Pb}$ & $26.74 \pm 0.57$ \\
\hline${ }^{241} \mathrm{Am}$ & $2.964 \pm 0.032$ \\
\hline
\end{tabular}

\section{UNCERTAINTIES}

The reported expanded uncertainty is based on a standard uncertainty multiplied by a coverage factor $k=2$, providing a coverage probability of approximately $95 \%$. The uncertainty evaluation has been carried out in accordance with UKAS requirements. 
NPL REPORT IR 59 


\section{CONTENTS}

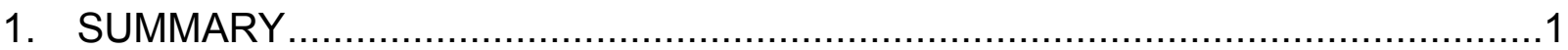

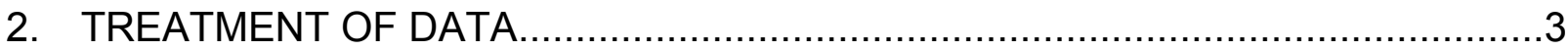

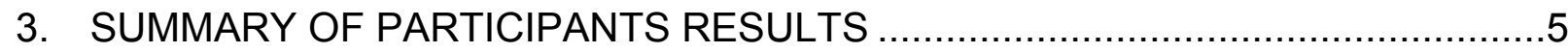

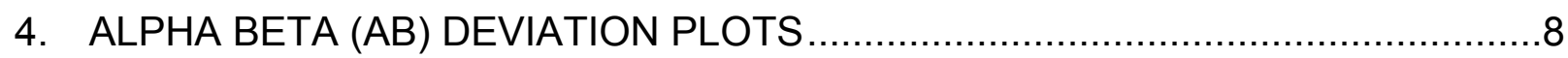

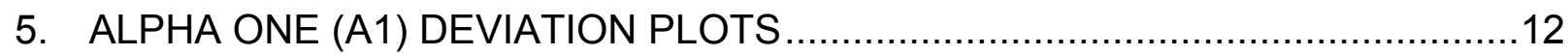

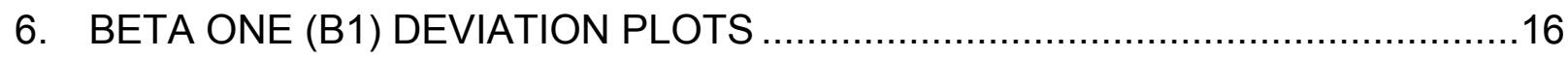

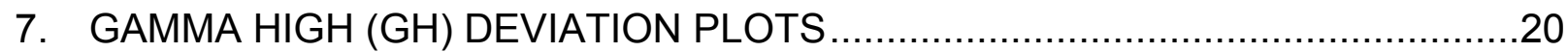

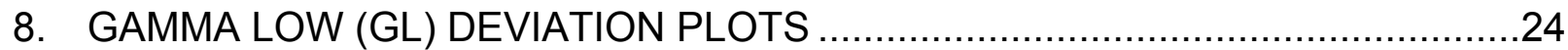

9. DEVIATION PLOTS AND TABULATED RESULTS ARRANGED

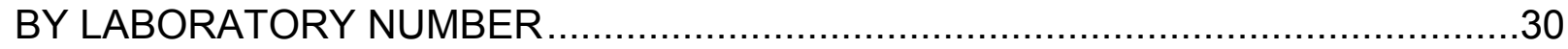

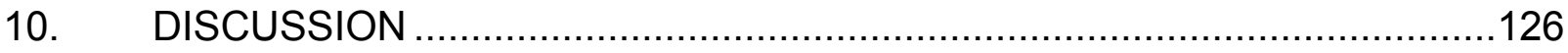

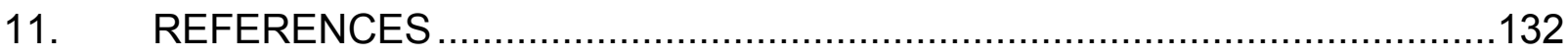

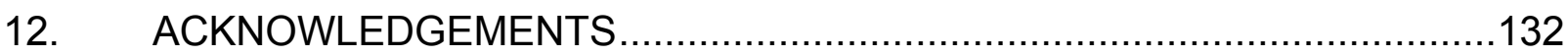

APPENDIX I GROSS MEASUREMENT RESULTS SUMMARY .........................133 
NPL REPORT IR 59 


\section{SUMMARY}

This Environmental Radioactivity Proficiency Test Exercise (PTE) was the twenty-sixth in a series of annual exercises run by NPL over the last 30 years. These exercises help analysts to identify metrology challenges and support UKAS accreditations in the quantification of radionuclides. A range of sample types were made available during previous exercises. These have been mostly aqueous but have on occasion included solid materials, which have been introduced subject to availability. This exercise consisted of aqueous solutions only with five sample types made available to the participants, summarised in Table 1.

Table 1 Summary of samples available to the participants for this proficiency test exercise.

\begin{tabular}{|c|c|c|c|c|}
\hline $\begin{array}{l}\text { Sample } \\
\text { type }\end{array}$ & $\begin{array}{c}\text { Sample } \\
\text { type } \\
\text { code }\end{array}$ & Contents & $\begin{array}{c}\text { Nominal } \\
\text { Mass } \\
\text { supplied }\end{array}$ & $\begin{array}{c}\text { Activity } \\
\text { range }\end{array}$ \\
\hline Alpha Beta & $A B$ & $\begin{array}{l}\text { one alpha- and two beta-emitting } \\
\text { radionuclides in dilute nitric acid }\end{array}$ & $20 \mathrm{~g}$ & $1-20 \mathrm{~Bq} \mathrm{~g}^{-1}$ \\
\hline Alpha One & $\mathrm{A} 1$ & $\begin{array}{l}\text { Three alpha-emitting radionuclides } \\
\text { in dilute nitric acid }\end{array}$ & $500 \mathrm{~g}$ & $1-20 \mathrm{~Bq} \mathrm{~kg}^{-1}$ \\
\hline Beta One & B1 & $\begin{array}{l}\text { Three beta-emitting radionuclides } \\
\text { in } 0.01 \mathrm{M} \mathrm{NaOH} \text { solution }\end{array}$ & $500 \mathrm{~g}$ & $0.1-2 \mathrm{~Bq} \mathrm{~g}^{-1}$ \\
\hline $\begin{array}{l}\text { Gamma } \\
\text { High }\end{array}$ & $\mathrm{GH}$ & $\begin{array}{l}\text { Three gamma-ray emitting } \\
\text { radionuclides in dilute nitric acid }\end{array}$ & $100 \mathrm{~g}$ & $1-25 \mathrm{~Bq} \mathrm{~g}^{-1}$ \\
\hline Gamma Low & $\mathrm{GL}$ & $\begin{array}{l}\text { Five gamma-ray emitting } \\
\text { radionuclides in dilute nitric acid }\end{array}$ & $500 \mathrm{~g}$ & $1-50 \mathrm{~Bq} \mathrm{~kg}{ }^{-1}$ \\
\hline
\end{tabular}

As in previous years, the main objective was to assess the performance of the participating laboratories. NPL acted as the exercise coordinator, preparing, and distributing the samples to participants who identified and quantified the activity per unit mass of the radionuclides present in the samples. NPL then collected, analysed, and interpreted the results which were compiled into a report.

Each participant was allocated by NPL a unique laboratory code number (if not already allocated in a previous PTE in this series). This was done in confidence so that no third parties could identify the participants by their allocated code number. The participants were asked to add their code numbers to their Reporting Forms, and the code numbers would be used by NPL to label the results in the final PTE report.

Each sample type was prepared in bulk by combining weighed aliquots of radioactive standards with a weighed amount of carrier solution and then diluting the mixture further to achieve the target activity per unit mass. Dilution factors were measured gravimetrically and were verified radiometrically through either liquid scintillation counting or high-purity germanium (HPGe) gamma spectrometry. The Assigned Value for each radionuclide was calculated from the division of the standardised activity per unit mass of the original standard solution by the dilution factor(s). The activities per unit mass of the radionuclides in the aqueous sample types were traceable to national standards of radioactivity, and therefore to the international measurement system.

The standard uncertainty of the Assigned Values for each radionuclide was derived from the uncertainty components attributed to the activity of the standardised parent solution, the 
gravimetric dilution and the decay correction to the reference time. These uncertainties have been evaluated and validated in accordance with the requirements of UKAS.

Throughout this report, unless otherwise stated, all uncertainties quoted in this report are combined standard uncertainties with no coverage factor applied; the corresponding confidence interval is approximately $68 \%$.

The bulk solution was subdivided into (typically) 50 bottles and homogeneity was checked by gamma spectrometry where applicable. Solution stability was checked by counting one or more bottles of each sample type at NPL at regular intervals throughout the course of the PTE; all solutions were found to be stable.

Participants' data were analysed to provide the deviation, and the associated stand uncertainty, from the assigned value. The participants' performance was then assessed using the method described in section 2.

After receipt of the results from the participants, the Power-Moderated Mean (PMM, Pommé, 2012) was calculated for each radionuclide/radionuclide type. This provides a more robust estimate than the weighted mean in the event of discrepant data sets. For mutually consistent data, the method approaches the weighted mean, the weights being the reciprocals of the variances associated with the measured values. For data suspected of inconsistency, the weighting is moderated by augmenting laboratory variances by a common amount and/or by decreasing the power of weighting factors. For increasingly discrepant data sets, there is a smooth transition from the weighted mean to the arithmetic mean.

The PMM was also calculated for the following quantities:

- Sample Type AB gross beta

- Sample Type B1 gross beta

- Sample Type A1 gross alpha

There were no cases where the PMM was used as the Assigned Value. Note that consensus values based on the PMM are not traceable to national standards of radioactivity. The PMM of the gross measurements is provided as an indicator and has not been used for performance assessment. It is for this reason results for gross measurements do not appear in the main body of the report. The gross measurements are given in APPENDIX I.

The dispatch of the samples was subcontracted to the following organisations:

The Courier Company (UK) Limited

11 James Way

Marshall Court

Milton Keynes MK1 1SU

Circle Express

Unit 1

Polar Park

Bath Rd

West Drayton UB7 0EX 


\section{TREATMENT OF DATA}

The data were analysed using the same methods as in the 2019 exercise (van Es et al., 2021). The deviation ' $D$ ' from the assigned value from each laboratory value was calculated from:

$$
D=\frac{L-N}{N}=\left(\frac{L}{N}-1\right)
$$

The standard uncertainty ' $u_{\mathrm{D}}$ ' of the deviation was calculated from:

$$
u_{D}=\frac{L}{N} \sqrt{\left(\frac{u_{L}}{L}\right)^{2}+\left(\frac{u_{N}}{N}\right)^{2}}
$$

The quantities zeta $(\zeta)$, the relative standard uncertainty of a laboratory's value $\left(R_{\mathrm{L}}\right)$ and the z-score were calculated from:

$$
\begin{aligned}
& \zeta=\frac{L-N}{\sqrt{u_{L}^{2}+u_{N}^{2}}} \\
& R_{L}=\frac{u_{L}}{L} \\
& Z=\frac{L-N}{\sigma_{p}}=\frac{L-N}{0.05823 N}
\end{aligned}
$$

where:

$L$ is the participant's value;

$N$ is the Assigned Value;

$u_{L}$ is the standard uncertainty of the participants' value;

$u_{N}$ is the standard uncertainty of the Assigned Value;

$\sigma_{\mathrm{p}}$ is the standard uncertainty for proficiency assessment. 
The value of the standard uncertainty for proficiency assessment $\sigma_{p}$ is chosen by perception (viz. ISO 13528:2015 paragraph 6.3). It corresponds to a level of performance that NPL would wish laboratories to be able to achieve. It corresponds to a deviation $D$ of $15 \%$ (at a $99 \%$ confidence level). In other words, any result with a deviation $D$ smaller than $\pm 15 \%$ will pass the z-test.

Note that the z-score presented is as defined in ISO 13528:2015 rather than the commonly understood z-score and is used to reject results based on a maximum percentage deviation.

The zeta and z-scores were used to determine whether the difference between the participant's value and the Assigned Value was significantly different from zero. The Interquartile Range outlier test (Harms and Gilligan, 2011) was used to determine whether the relative uncertainty $R_{\mathrm{L}}$ was significantly larger than the other values in the data set. Note that this test is unable to identify outliers if the data set is smaller than seven.

Results for which the absolute values of the zeta score and the $z$-score are both $\leq 2.576$ and for which $R_{\mathrm{L}}$ is not significantly larger than the other values in the data set are taken to mean that the participant's value is 'in agreement' with the Assigned Value. These results are plotted in white in this report.

If (i) $R_{\mathrm{L}}$ is significantly larger than the other values in the data set, or (ii) the result passes the zeta test but not the z-test (i.e., there is a large deviation from the Assigned Value combined with a large uncertainty), or (iii) the result passes the z-test but not the zeta test (where the deviation is less than $15 \%$ from the Assigned Value but the standard uncertainty is insufficient result in agreement with the Assigned Value), the participant's value is classified as 'questionable' (plotted in yellow).

If the absolute values of both the zeta score and the z-score are greater than 2.576, then the participant's value is classified as 'discrepant' from the Assigned Value (plotted in red), regardless of the value of $R_{\mathrm{L}}$.

A result was only classified as 'in agreement' when the three tests (the zeta test, the relative uncertainty outlier test and the z-test) were passed. A failure to pass one of these tests resulted in a classification 'questionable'. Failure of both the zeta test and the z-test resulted in a classification 'discrepant'. The classification criteria used to assess the performance of participants' is summarised in Table 2.

Table 2 Summary of data classification criteria

\begin{tabular}{|c|c|c|c|}
\hline zeta test & $\boldsymbol{R}_{\mathbf{L}}$ test & $\boldsymbol{z}$ test & Classification \\
\hline pass & pass & pass & in agreement \\
\hline pass & fail & pass & questionable \\
\hline fail & pass & pass & questionable \\
\hline pass & - & fail & questionable \\
\hline fail & - & fail & discrepant \\
\hline
\end{tabular}




\section{SUMMARY OF PARTICIPANTS RESULTS}

The summary of classification results for each radionuclide in each sample type are provided in Table 3. The number of no results is for indication only and does not relate to a failed result as it is considered that all radionuclides in a sample may not be relevant for a particular participant and that they have only reported for those radionuclides relevant to their test regimes.

Table 3 Summary of classifications for each radionuclide in each sample type

\begin{tabular}{|c|c|c|c|c|c|}
\hline Radionuclide & Samples despatched & Pass & Questionable & Fail & No result \\
\hline \multicolumn{6}{|c|}{ AB } \\
\hline${ }^{3} \mathrm{H}$ & \multirow{3}{*}{27} & 16 & 3 & 3 & 3 \\
\hline${ }^{90} \mathrm{Sr}$ & & 19 & 1 & 1 & 2 \\
\hline${ }^{238} \mathrm{Pu}$ & & 18 & 0 & 1 & 4 \\
\hline \multicolumn{6}{|c|}{ A1 } \\
\hline${ }^{233} \mathrm{U}$ & \multirow{3}{*}{15} & 7 & 1 & 3 & 3 \\
\hline${ }^{238} \mathrm{Pu}$ & & 7 & 3 & 4 & 1 \\
\hline${ }^{243} \mathrm{Am}$ & & 7 & 2 & 5 & 3 \\
\hline \multicolumn{6}{|c|}{ B1 } \\
\hline${ }^{3} \mathrm{H}$ & \multirow{3}{*}{28} & 24 & 3 & 3 & 3 \\
\hline${ }^{14} \mathrm{C}$ & & 16 & 2 & 2 & 8 \\
\hline 129 & & 11 & 5 & 1 & 11 \\
\hline \multicolumn{6}{|c|}{ GH } \\
\hline${ }^{60} \mathrm{Co}$ & \multirow{3}{*}{28} & 23 & 3 & 1 & 0 \\
\hline${ }^{133} \mathrm{Ba}$ & & 22 & 3 & 1 & 1 \\
\hline${ }^{154} \mathrm{Eu}$ & & 23 & 2 & 2 & 0 \\
\hline \multicolumn{6}{|c|}{ GL } \\
\hline${ }^{134} \mathrm{Cs}$ & \multirow{5}{*}{30} & 24 & 3 & 0 & 1 \\
\hline${ }^{137} \mathrm{Cs}$ & & 26 & 1 & 0 & 1 \\
\hline${ }^{155} \mathrm{Eu}$ & & 26 & 0 & 1 & 1 \\
\hline${ }^{210} \mathrm{~Pb}$ & & 12 & 5 & 4 & 6 \\
\hline${ }^{241} \mathrm{Am}$ & & 18 & 9 & 0 & 2 \\
\hline
\end{tabular}


In addition to the analyses of individual participants' data as described in Section 2, the PMM of the reported results for each radionuclide was compared with the NPL Assigned Values. The results are given in Tables $4-8$. The tests as described in section 2 are used to assess the agreement between these values. The reference time is 2020-06-01 12:00:00 UTC.

Table 4 AB summary

\begin{tabular}{|c|c|c|c|c|c|}
\hline Radionuclide & $\begin{array}{l}\text { NPL Assigned Values } \\
\qquad\left(\mathrm{Bq} \mathrm{g}^{-1}\right)\end{array}$ & PMM (Bq g-1) & $\begin{array}{c}\text { Deviation } \\
\%\end{array}$ & Zeta & $\begin{array}{l}\text { Critical } \\
\text { Value }\end{array}$ \\
\hline${ }^{3} \mathrm{H}$ & $6.375 \pm 0.069$ & $6.383 \pm 0.095$ & 0.1 & 0.07 & 2.70 \\
\hline${ }^{90} \mathrm{Sr}$ & $3.865 \pm 0.010$ & $3.770 \pm 0.059$ & -2.5 & -1.60 & 2.86 \\
\hline${ }^{238} \mathrm{Pu}$ & $13.933 \pm 0.032$ & $14.18 \pm 0.15$ & 1.8 & 1.62 & 2.86 \\
\hline
\end{tabular}

Table 5 A 1 summary

\begin{tabular}{|c|c|c|c|c|c|}
\hline Radionuclide & $\begin{array}{c}\text { NPL Assigned Values } \\
\left(\mathrm{Bq} \mathrm{kg}^{-1}\right)\end{array}$ & PMM (Bq kg-1) & $\begin{array}{c}\text { Deviation } \\
\%\end{array}$ & Zeta & $\begin{array}{c}\text { Critical } \\
\text { Value }\end{array}$ \\
\hline${ }^{233} \mathrm{U}$ & $2.0723 \pm 0.0074$ & $2.03 \pm 0.10$ & -1.9 & -0.37 & 3.25 \\
\hline${ }^{238} \mathrm{Pu}$ & $6.485 \pm 0.020$ & $6.99 \pm 0.25$ & 7.8 & 1.98 & 3.06 \\
\hline${ }^{243} \mathrm{Am}$ & $4.476 \pm 0.046$ & $4.65 \pm 0.19$ & 3.8 & 0.87 & 3.01 \\
\hline
\end{tabular}

Table 6 B1 summary

\begin{tabular}{|c|c|c|c|c|c|}
\hline Radionuclide & $\begin{array}{c}\text { NPL Assigned Values } \\
\left(\mathbf{B q ~ 9}^{-1}\right)\end{array}$ & PMM (Bq 9 $\left.{ }^{-1}\right)$ & $\begin{array}{c}\text { Deviation } \\
\%\end{array}$ & Zeta & $\begin{array}{c}\text { Critical } \\
\text { Value }\end{array}$ \\
\hline${ }^{3} \mathrm{H}$ & $0.5815 \pm 0.0067$ & $0.5887 \pm 0.0043$ & 1.2 & 0.91 & 2.58 \\
\hline${ }^{14} \mathrm{C}$ & $0.3253 \pm 0.0015$ & $0.3161 \pm 0.0050$ & -2.8 & -1.76 & 2.88 \\
\hline${ }^{129}$ & $0.1941 \pm 0.0010$ & $0.1995 \pm 0.0050$ & 2.7 & 1.04 & 2.90 \\
\hline
\end{tabular}

Table $7 \mathrm{GH}$ summary

\begin{tabular}{|c|c|c|c|c|c|}
\hline Radionuclide & $\begin{array}{c}\text { NPL Assigned Values } \\
\left(\mathrm{Bq} \mathrm{g}^{-1}\right)\end{array}$ & $\mathrm{PMM}\left(\mathrm{Bq} \mathrm{g^{-1 }}\right)$ & $\begin{array}{c}\text { Deviation } \\
\%\end{array}$ & Zeta & $\begin{array}{l}\text { Critical } \\
\text { Value }\end{array}$ \\
\hline${ }^{60} \mathrm{Co}$ & $2.8224 \pm 0.0061$ & $2.818 \pm 0.022$ & -0.1 & -0.17 & 2.77 \\
\hline${ }^{133} \mathrm{Ba}$ & $19.24 \pm 0.14$ & $18.76 \pm 0.19$ & -2.5 & -2.05 & 2.67 \\
\hline${ }^{154} \mathrm{Eu}$ & $2.504 \pm 0.020$ & $2.495 \pm 0.020$ & -0.4 & -0.32 & 2.63 \\
\hline
\end{tabular}


NPL REPORT IR 59

Table 8 GL summary

\begin{tabular}{|c|c|c|c|c|c|}
\hline Radionuclide & $\begin{array}{c}\text { NPL Assigned Values } \\
\qquad\left(\mathrm{Bq} \mathrm{kg}^{-1}\right)\end{array}$ & PMM (Bq g $\left.{ }^{-1}\right)$ & $\begin{array}{l}\text { Deviation } \\
\%\end{array}$ & Zeta & $\begin{array}{l}\text { Critical } \\
\text { Value }\end{array}$ \\
\hline${ }^{134} \mathrm{Cs}$ & $10.23 \pm 0.11$ & $9.849 \pm 0.088$ & -3.7 & -2.71 & 2.58 \\
\hline${ }^{137} \mathrm{Cs}$ & $4.547 \pm 0.041$ & $4.592 \pm 0.054$ & 1.0 & 0.66 & 2.66 \\
\hline${ }^{155} \mathrm{Eu}$ & $24.43 \pm 0.57$ & $24.23 \pm 0.22$ & -0.8 & -0.33 & 2.58 \\
\hline${ }^{210} \mathrm{~Pb}$ & $26.74 \pm 0.28$ & $25.64 \pm 0.66$ & -4.1 & -1.55 & 2.85 \\
\hline${ }^{241} \mathrm{Am}$ & $2.964 \pm 0.016$ & $3.061 \pm 0.074$ & 3.3 & 1.28 & 2.76 \\
\hline
\end{tabular}




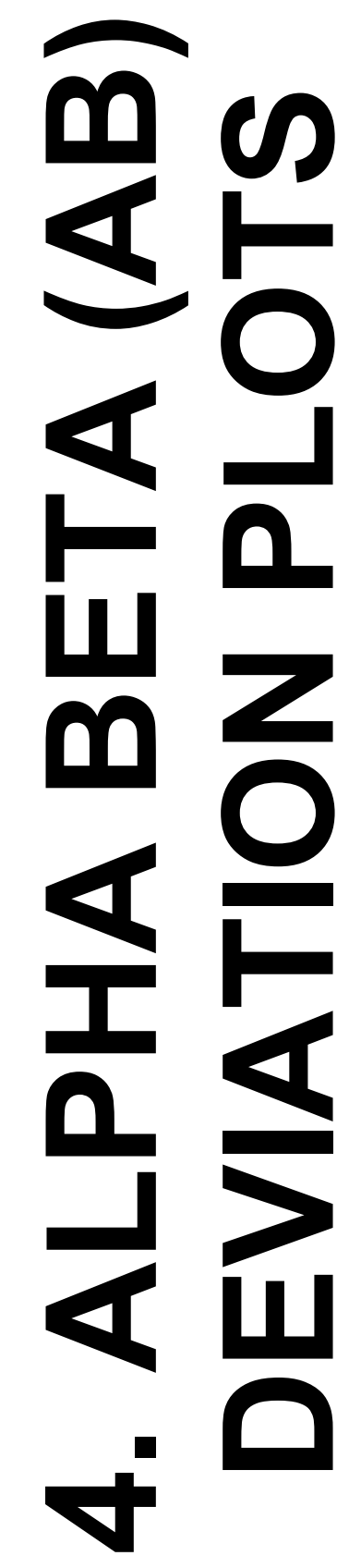

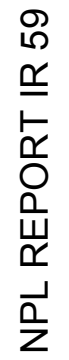




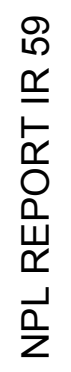

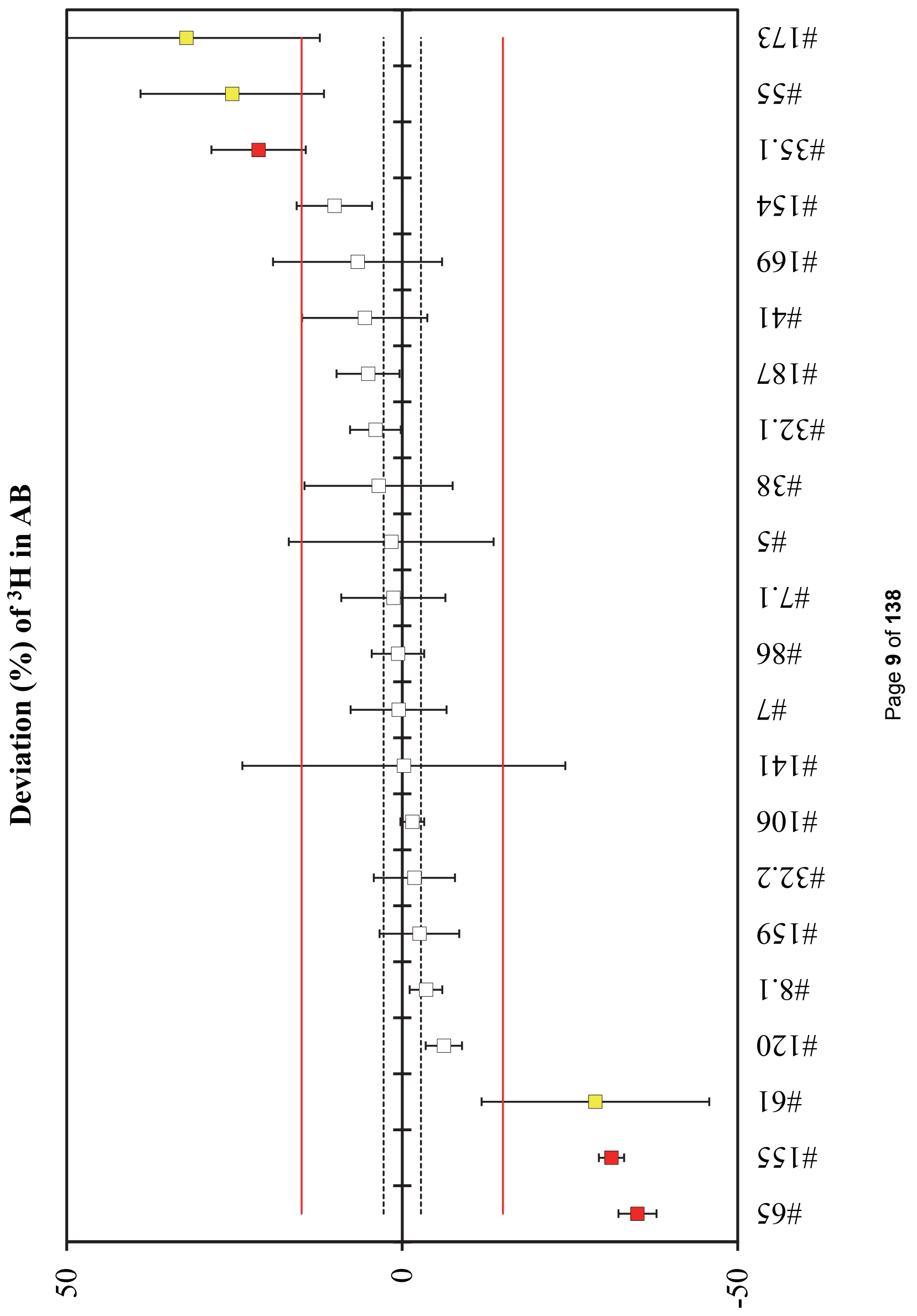




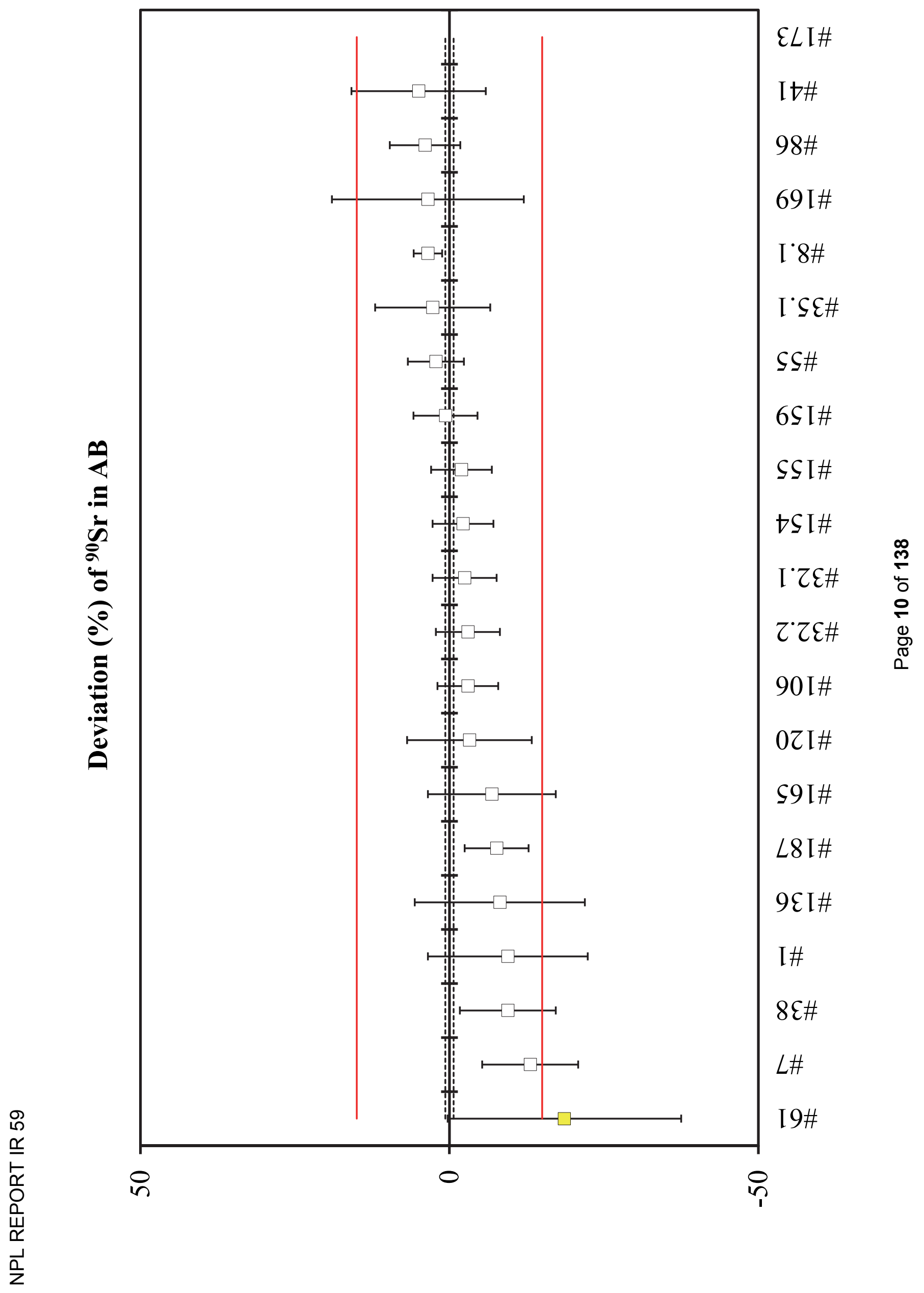




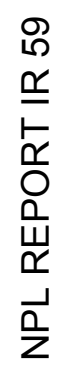

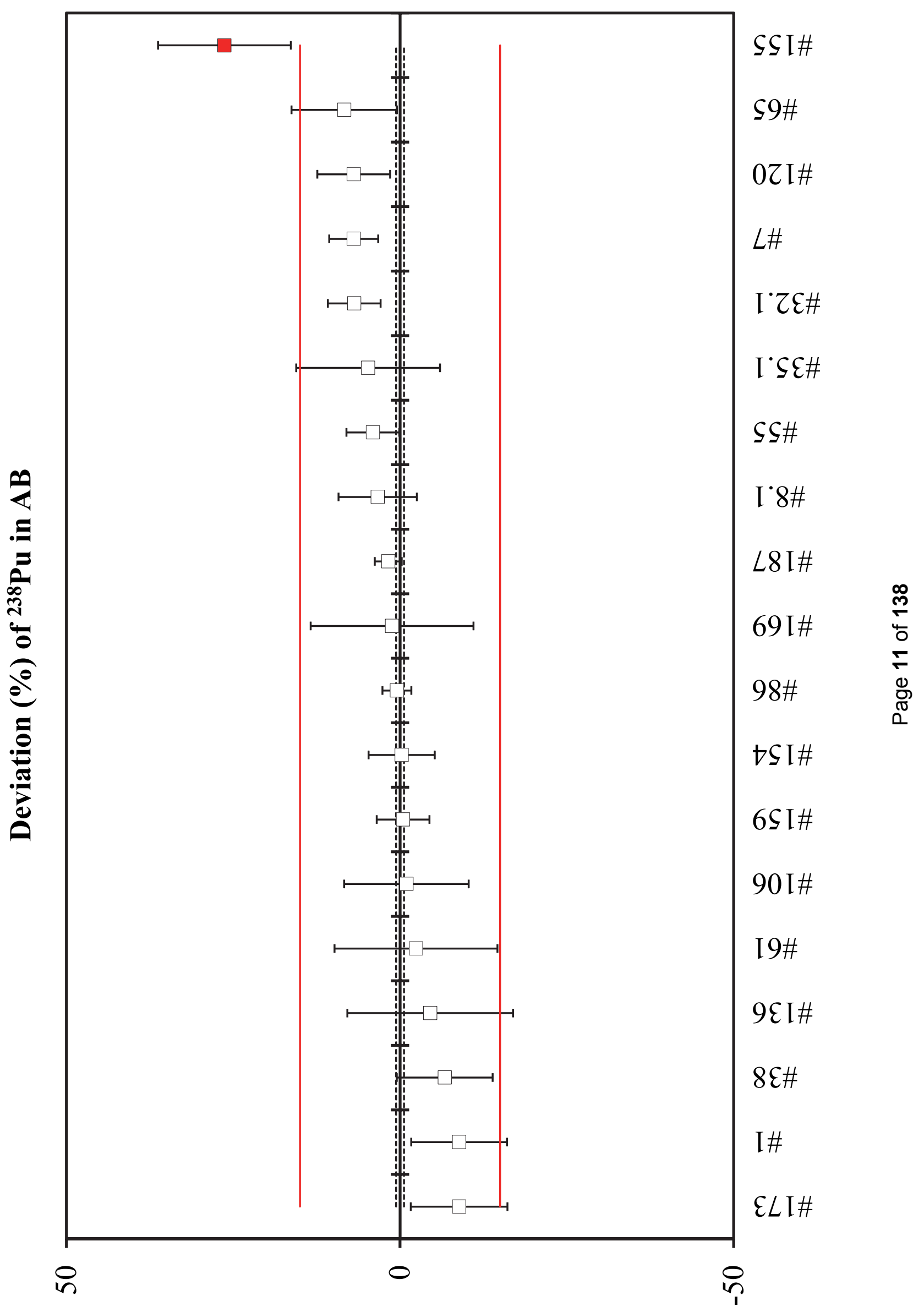




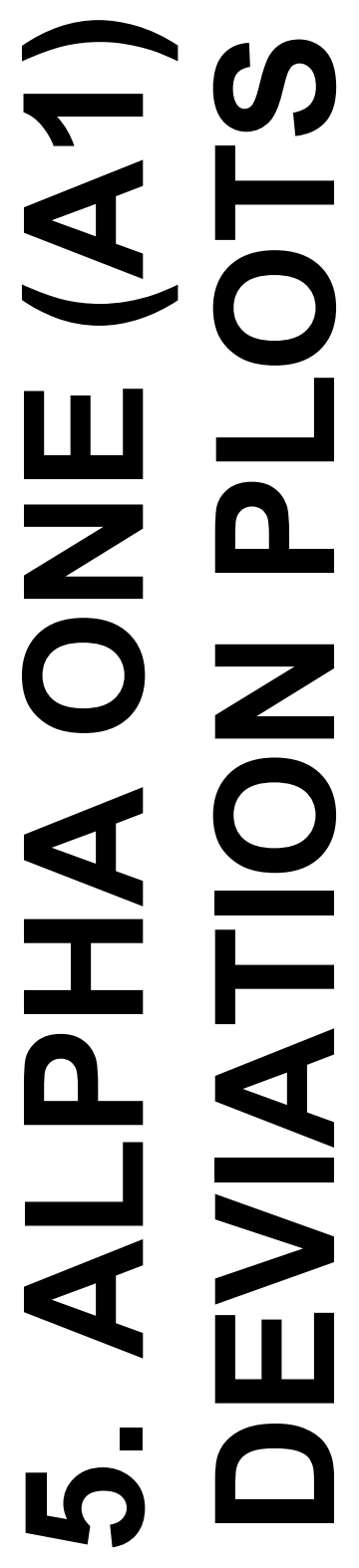

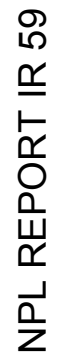




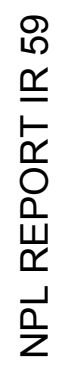

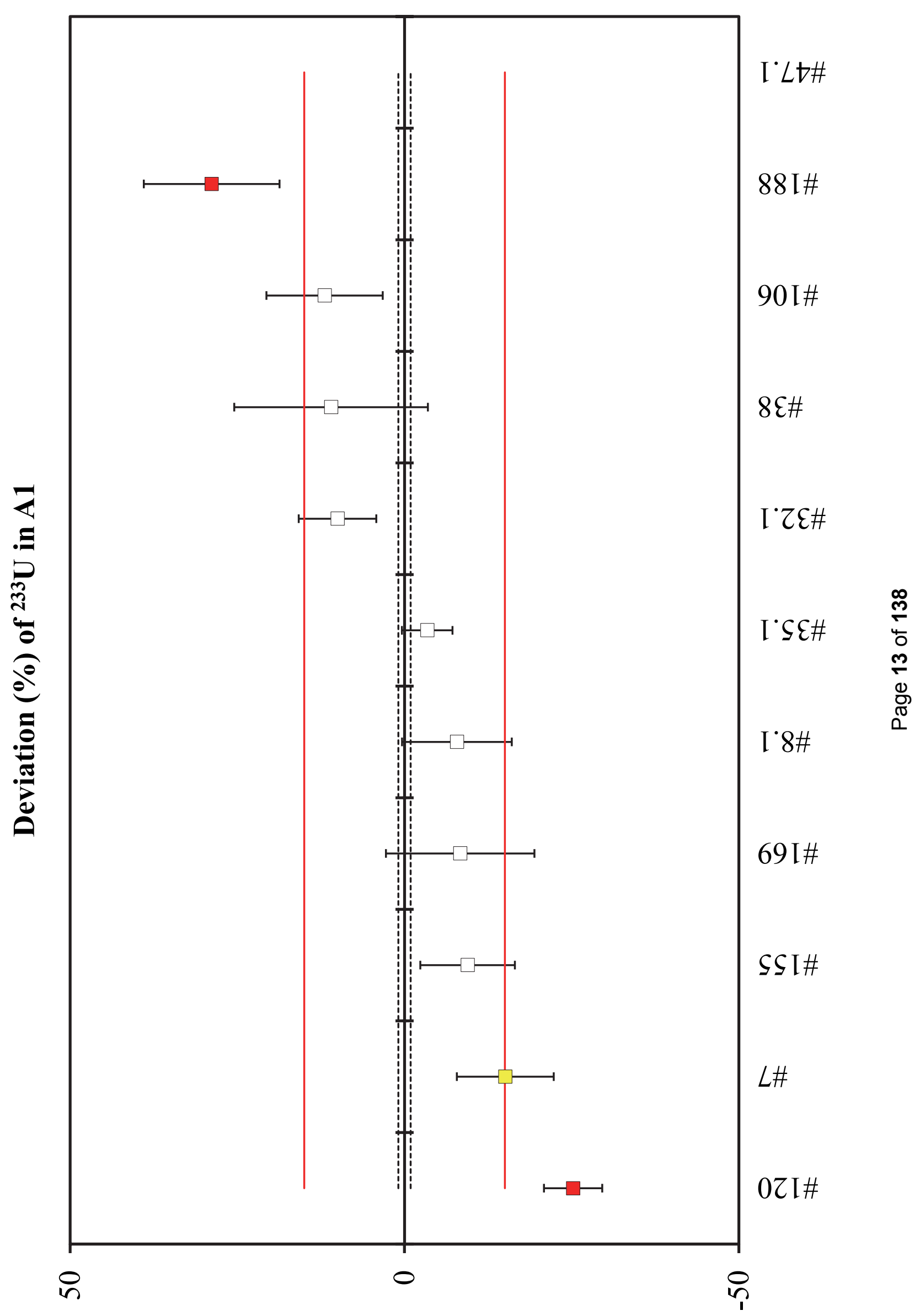




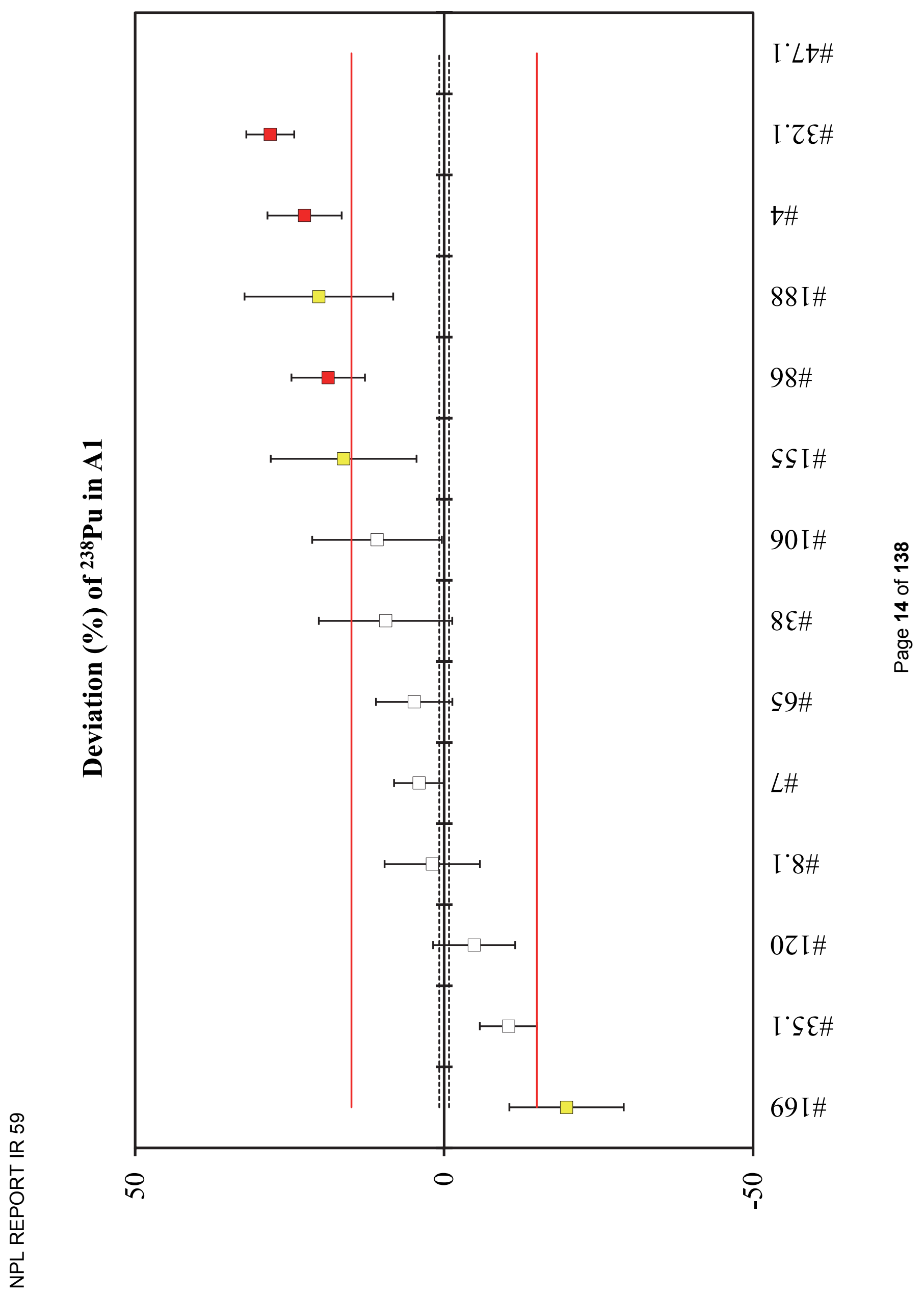




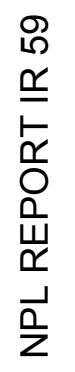

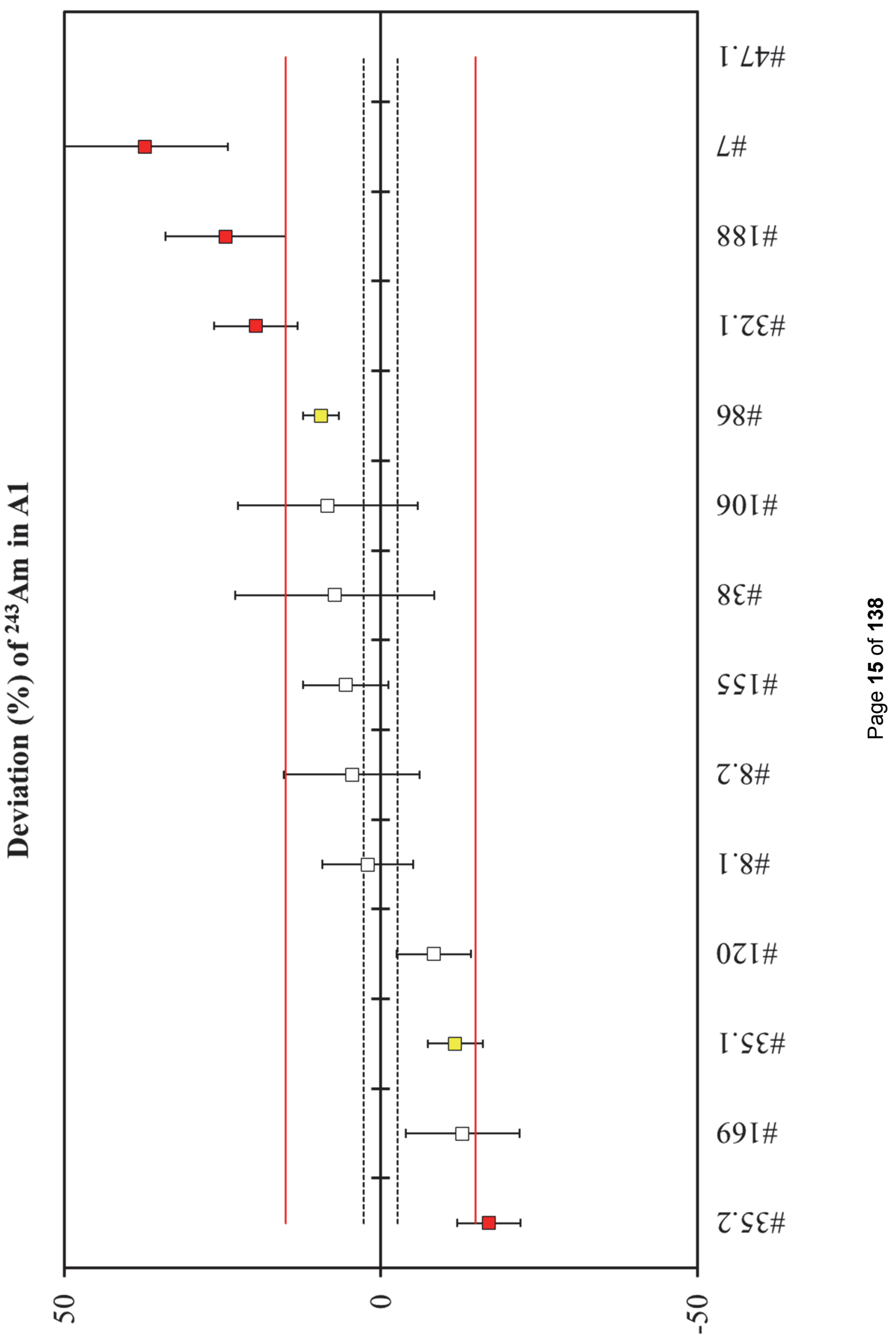




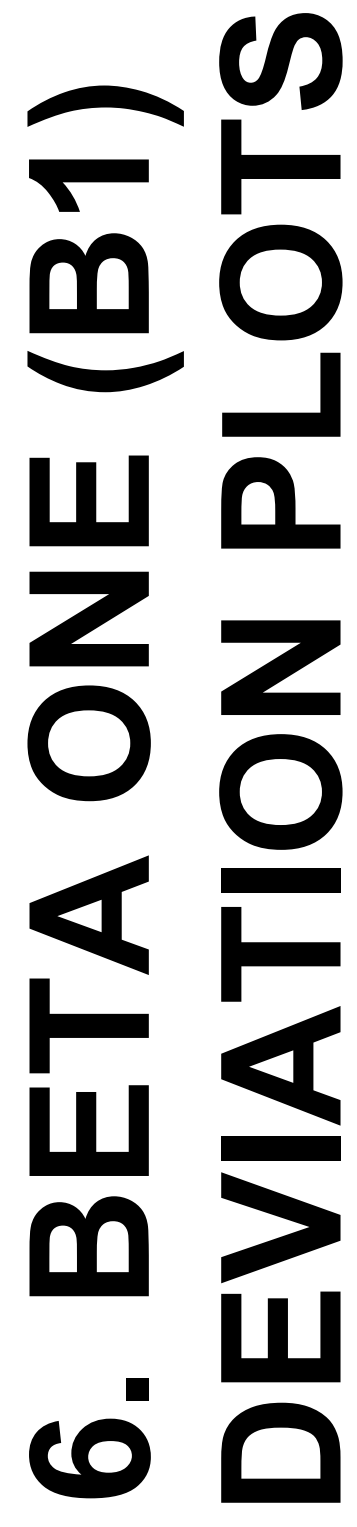

$\stackrel{0}{m}$
$\stackrel{2}{4}$
0
0
0
0
0
0

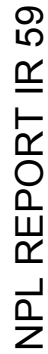




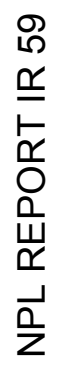

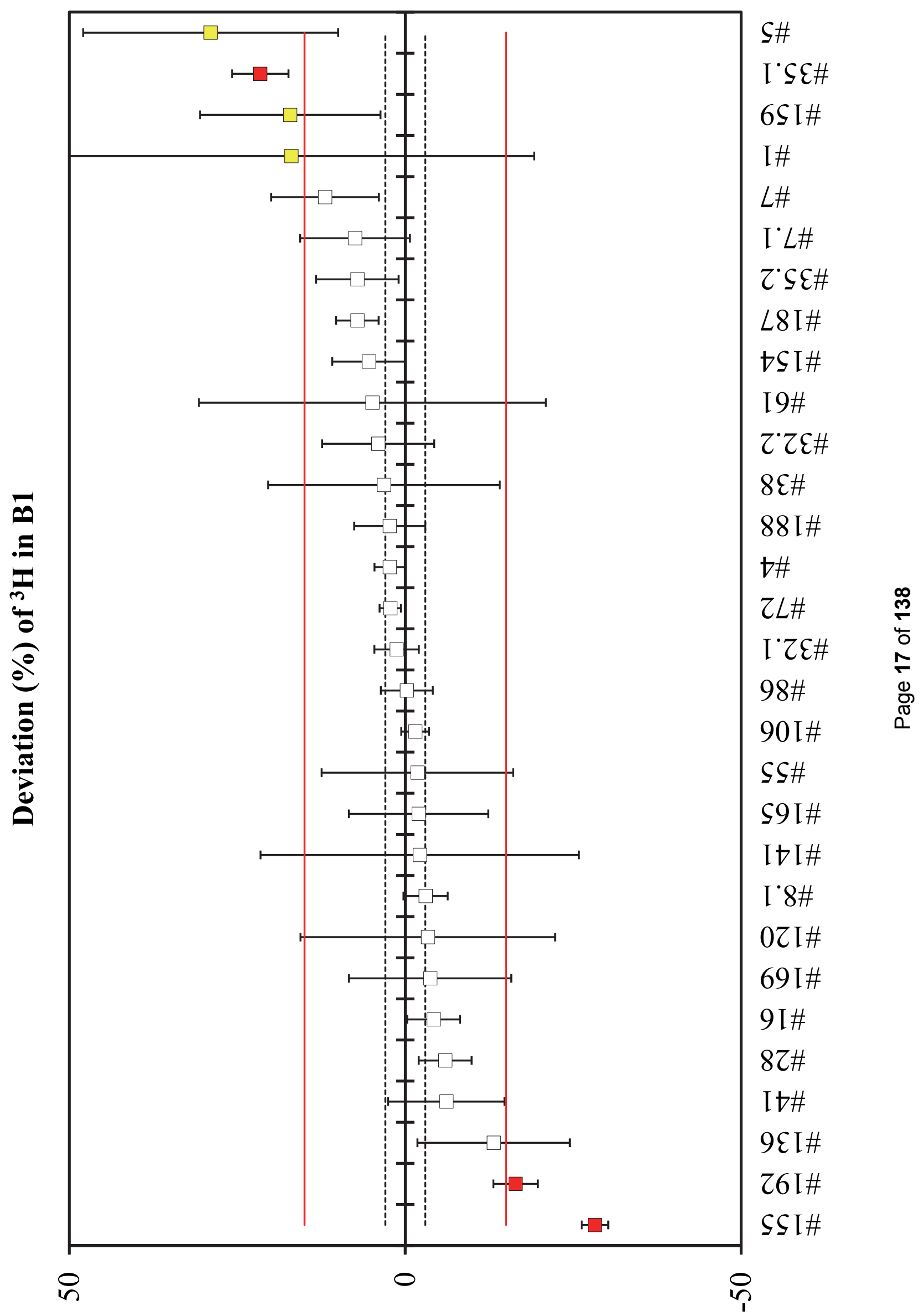




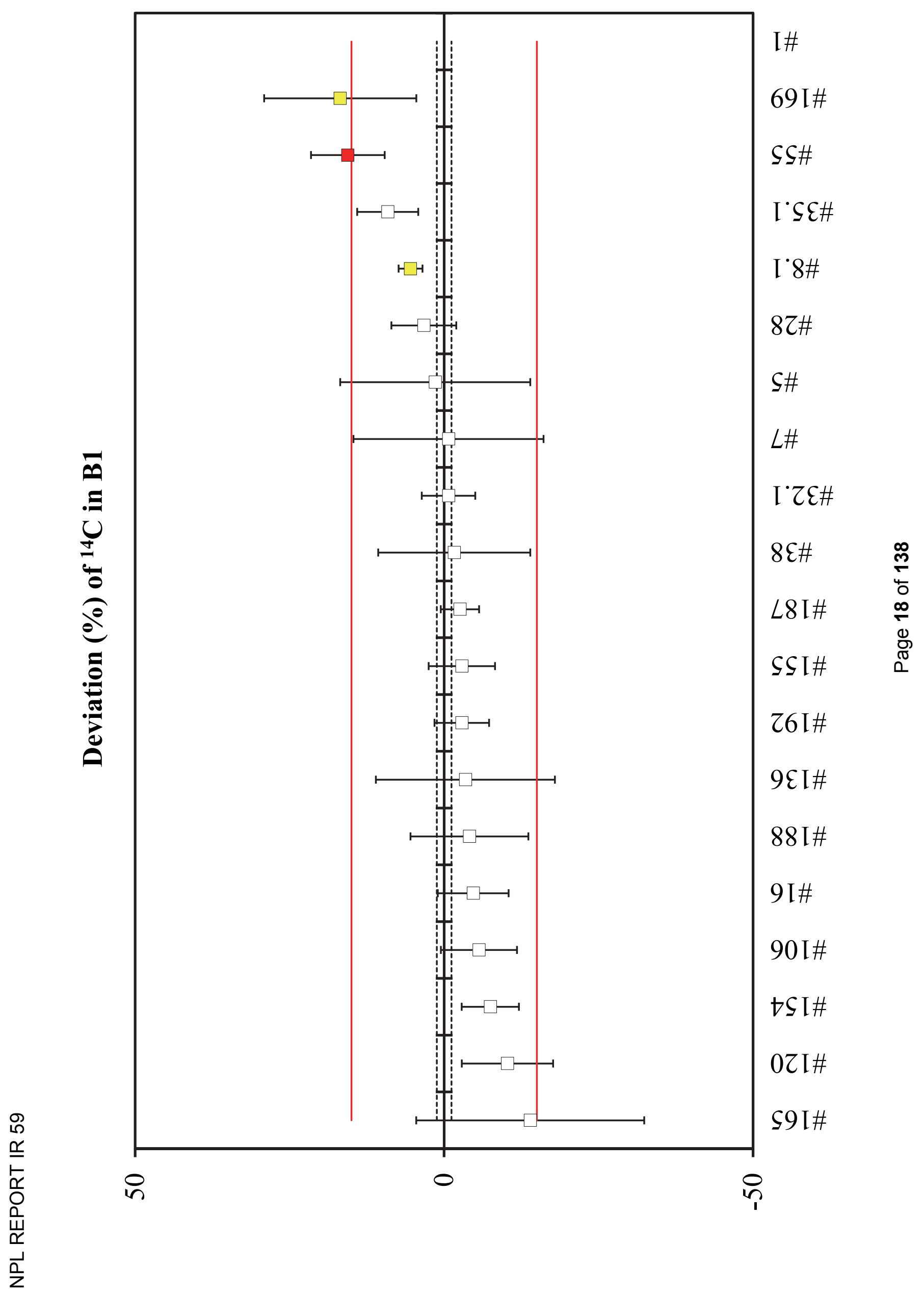




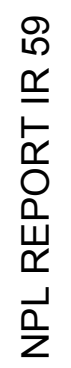

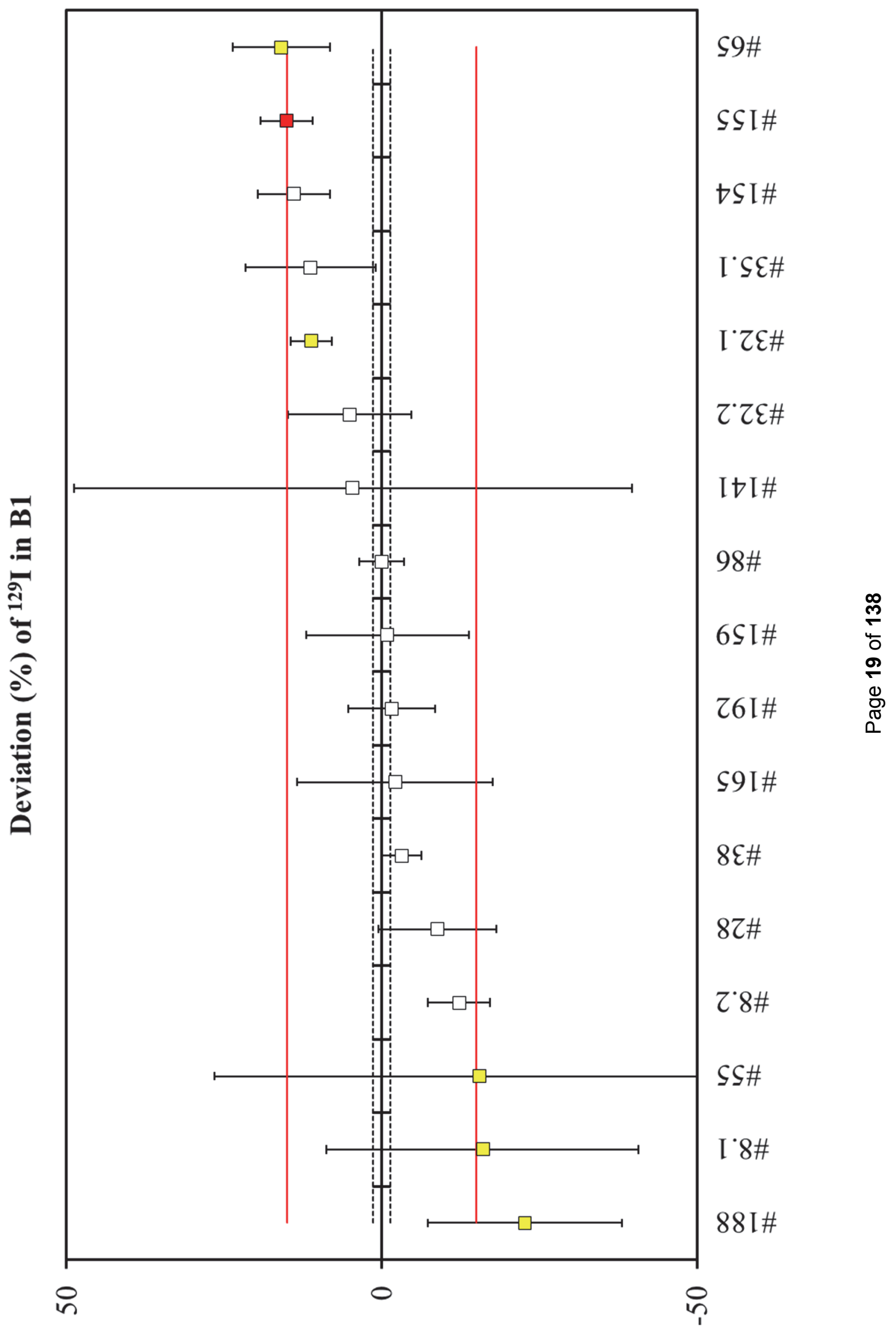




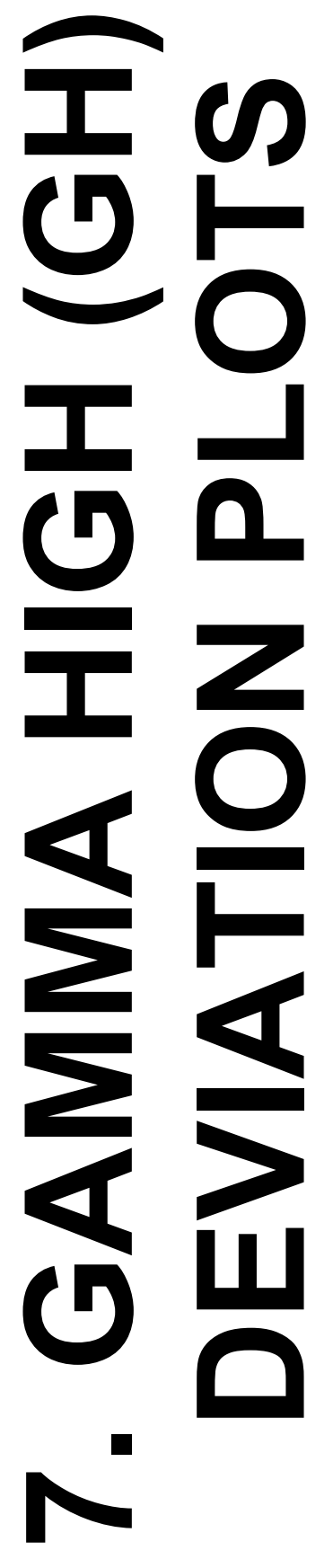

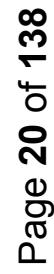

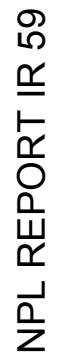




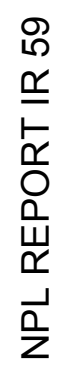

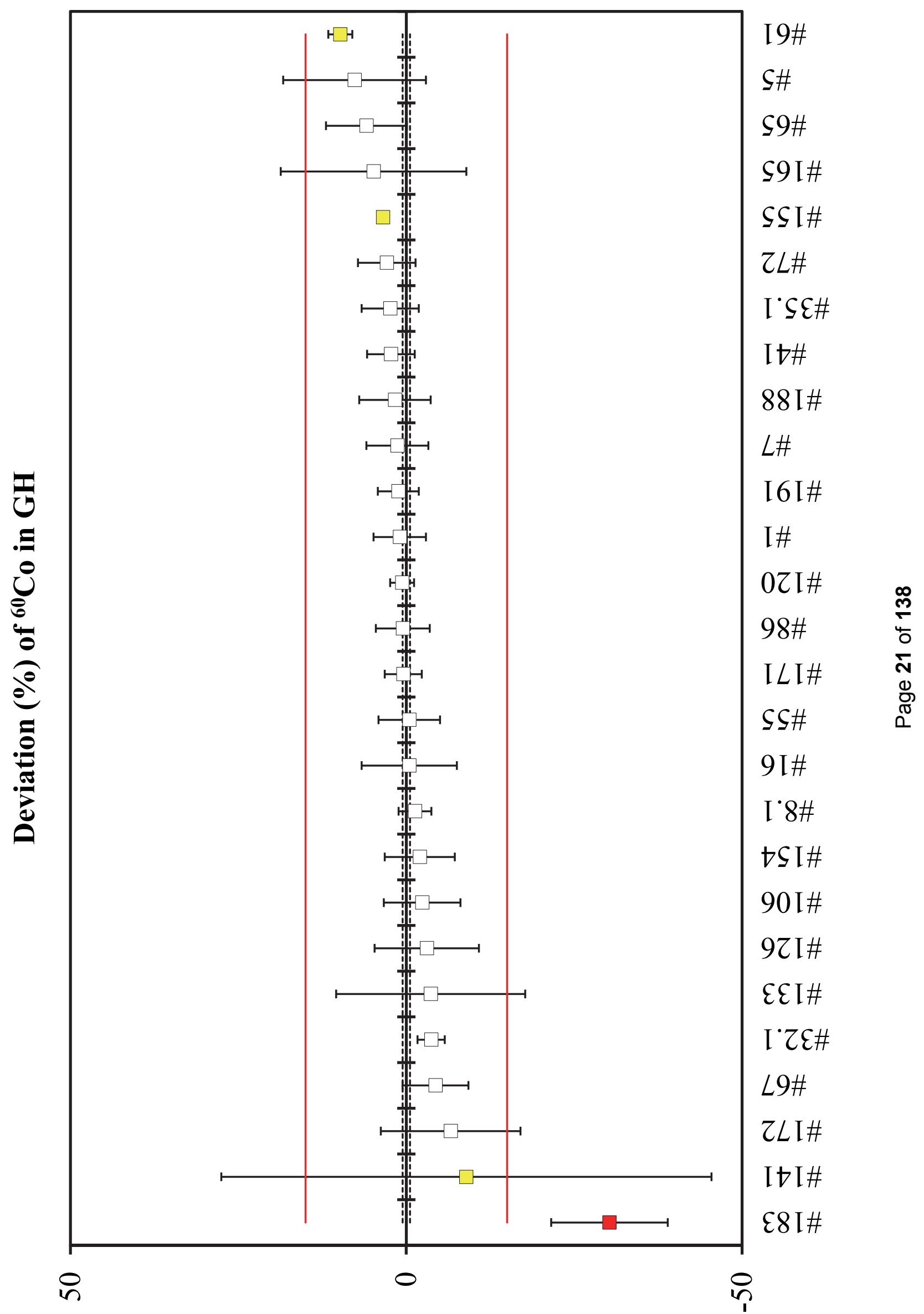




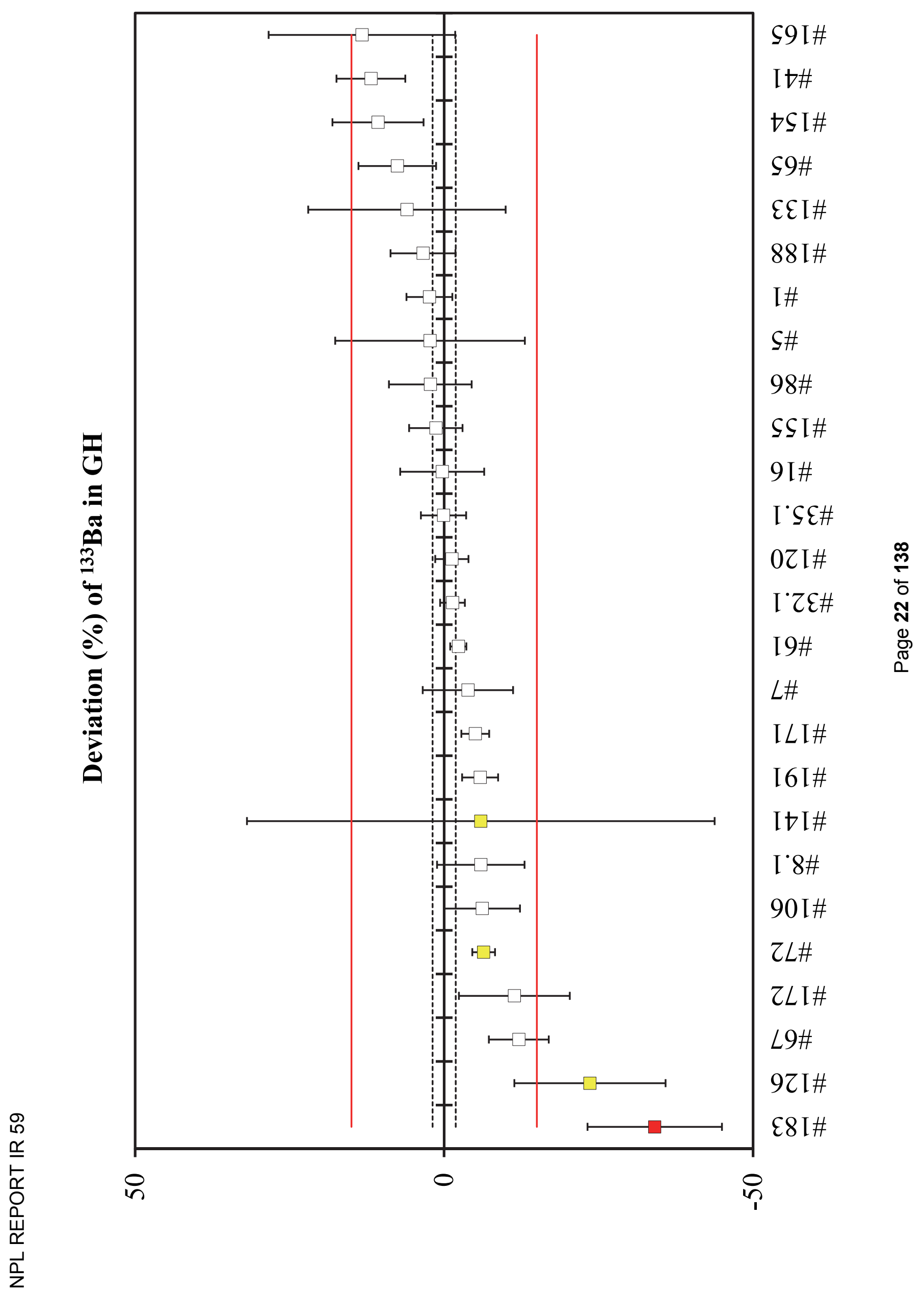




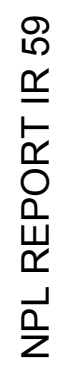

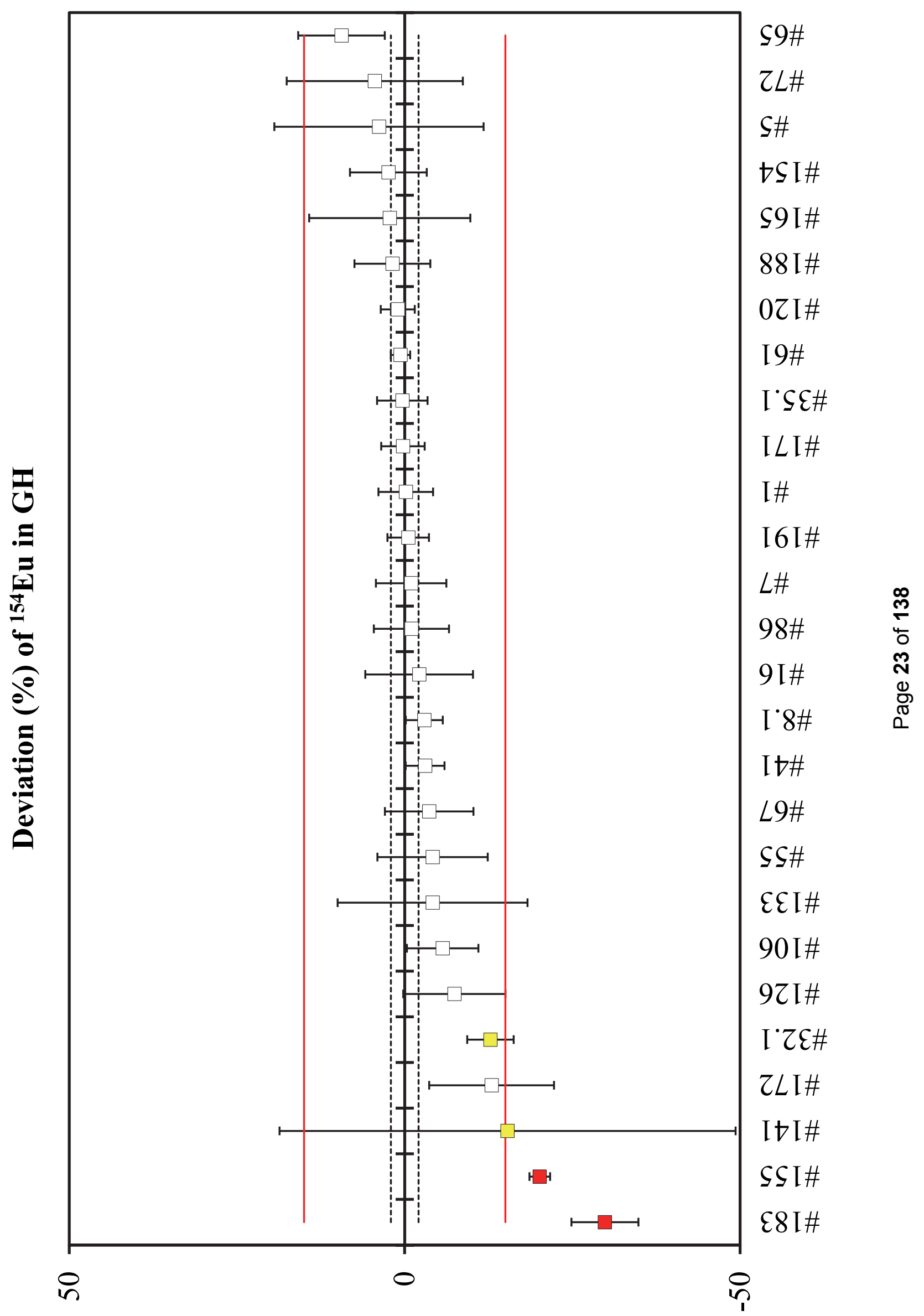




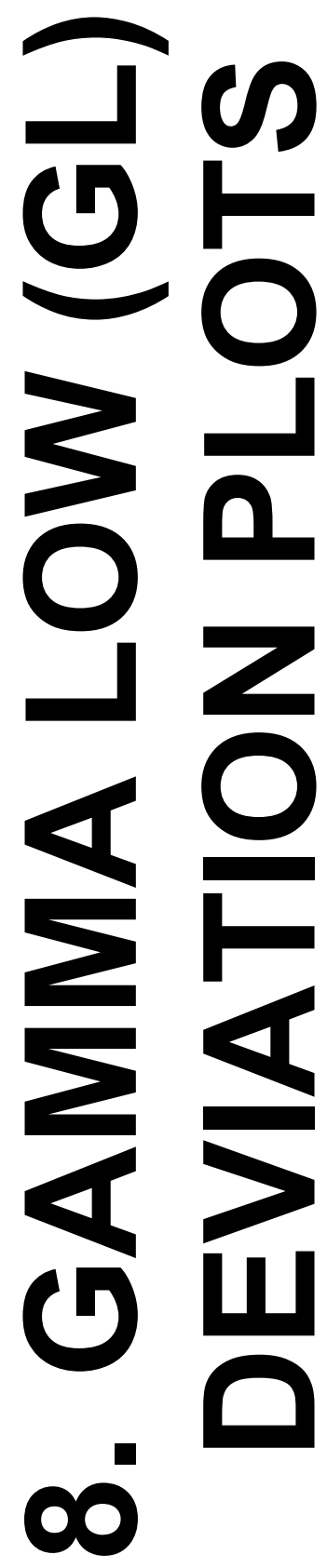

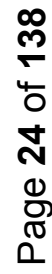

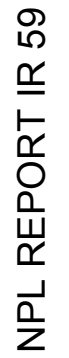




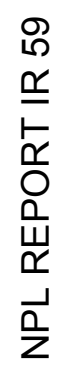

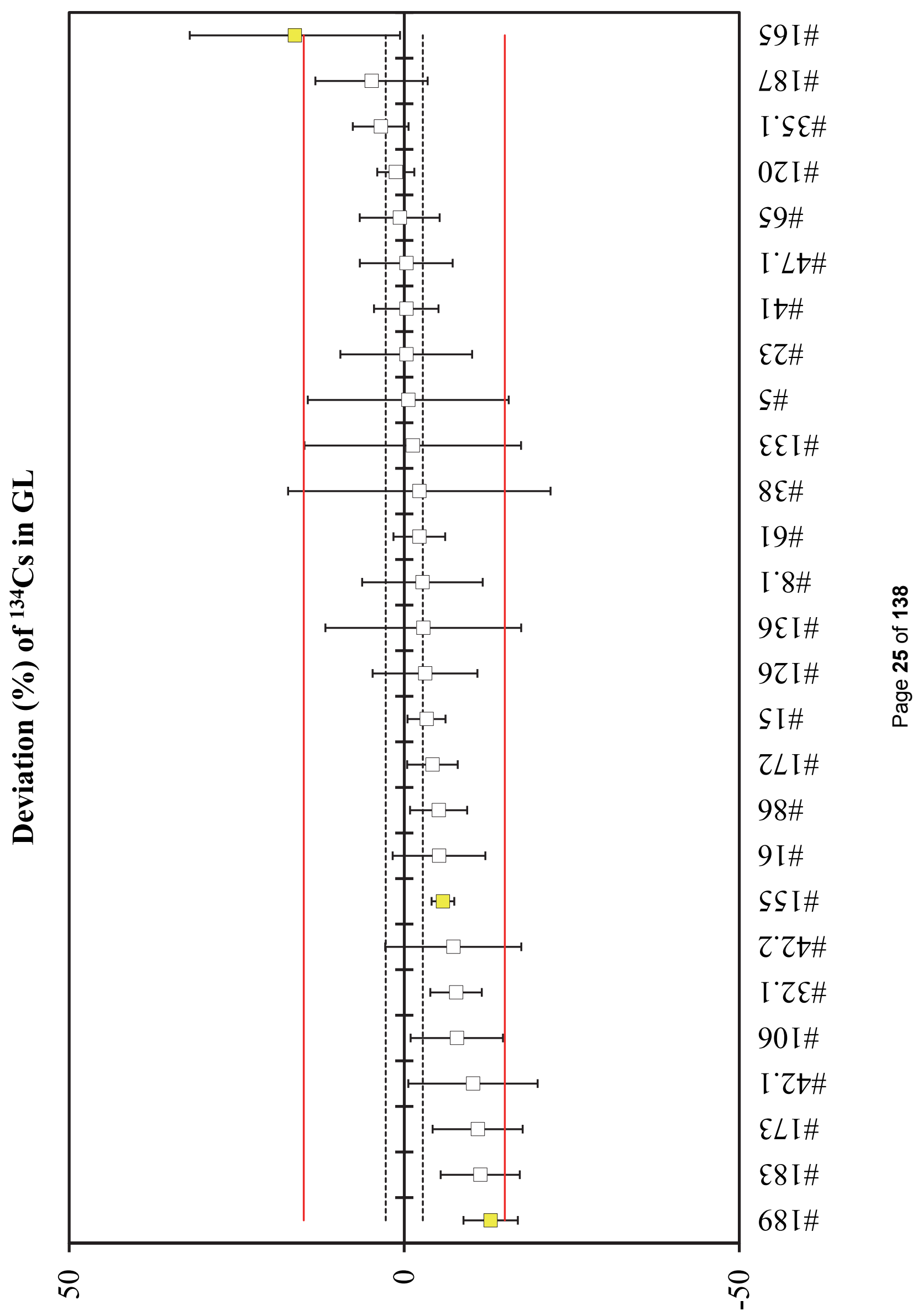




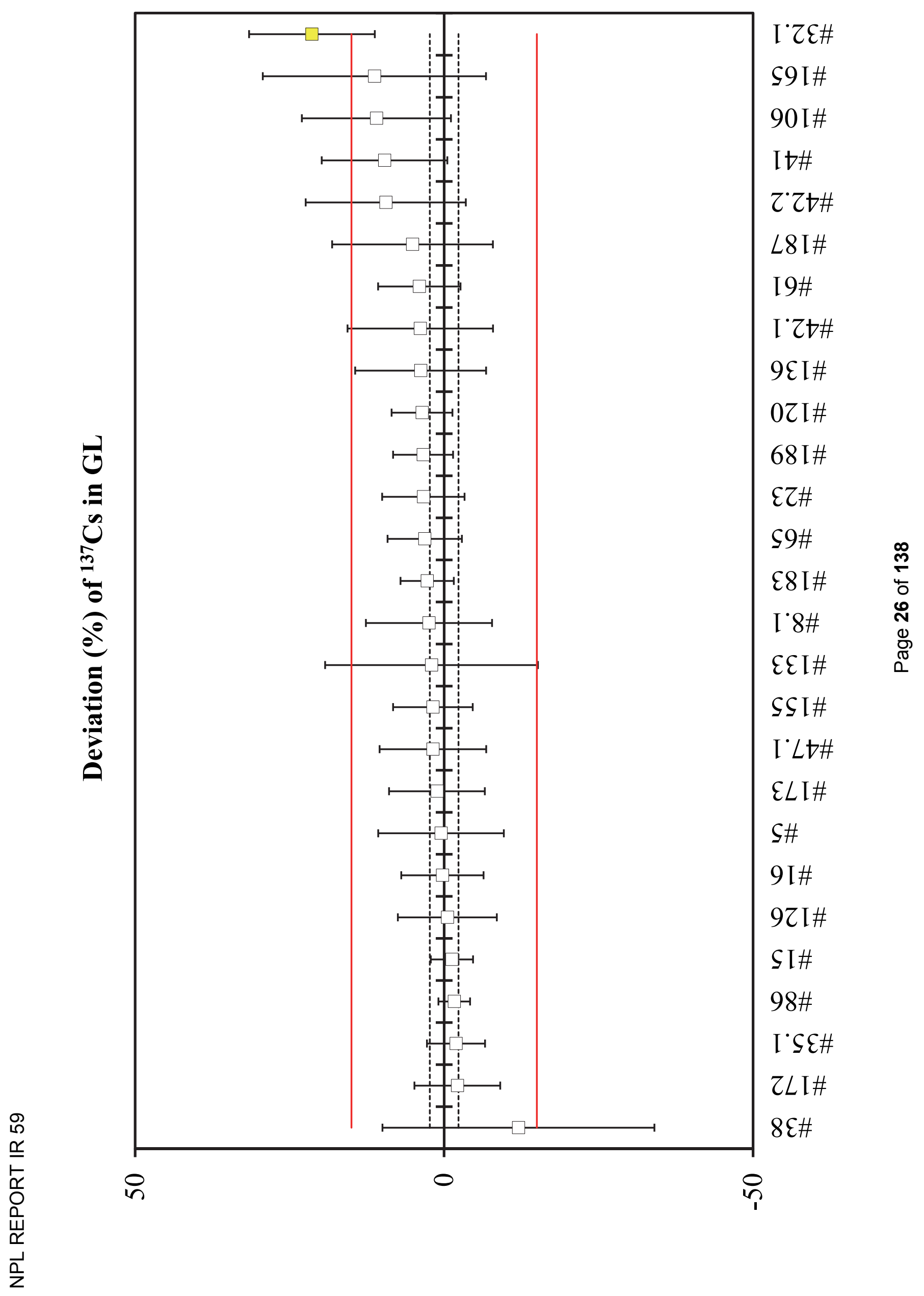




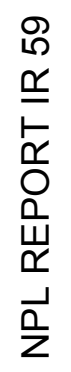

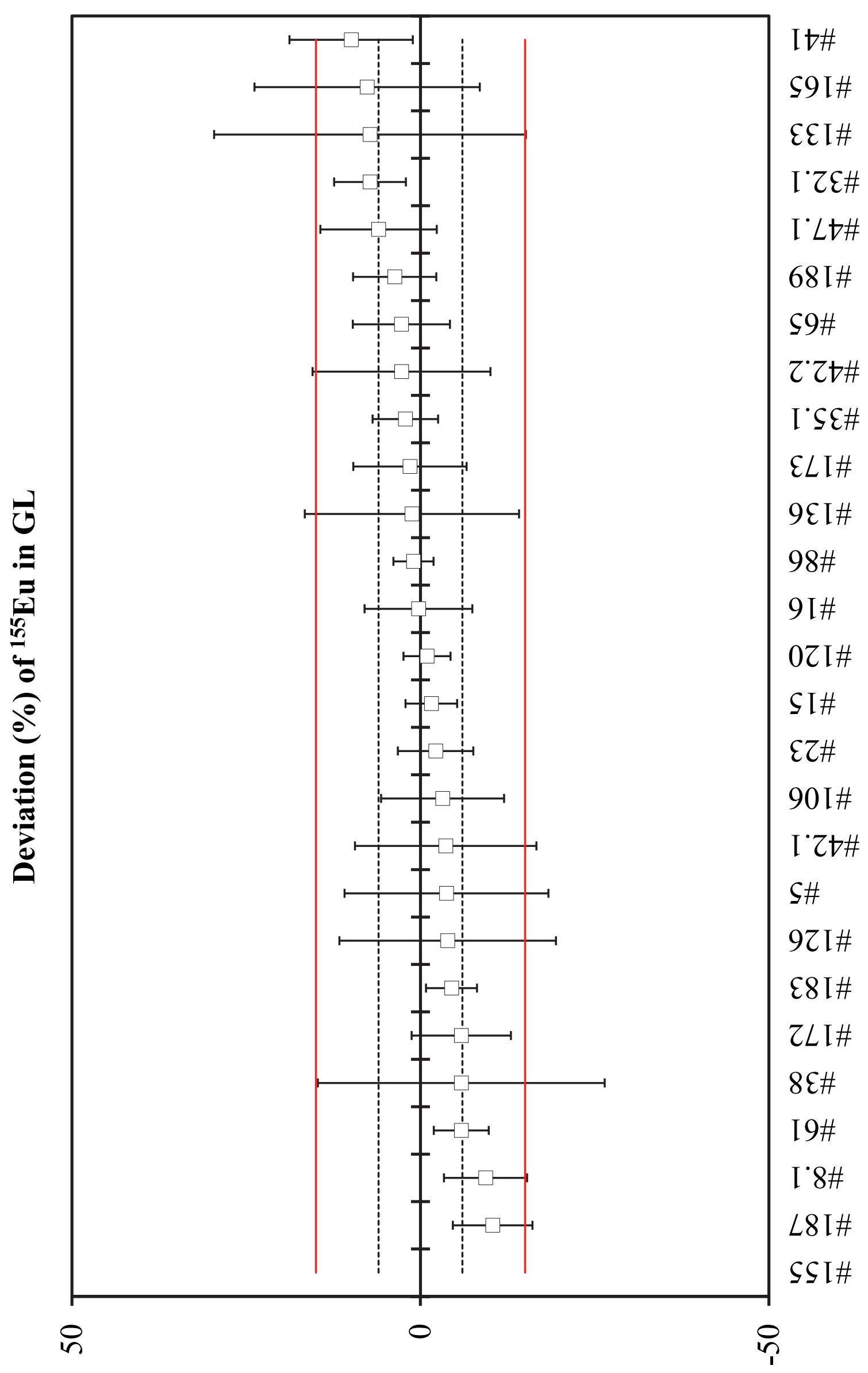

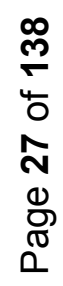




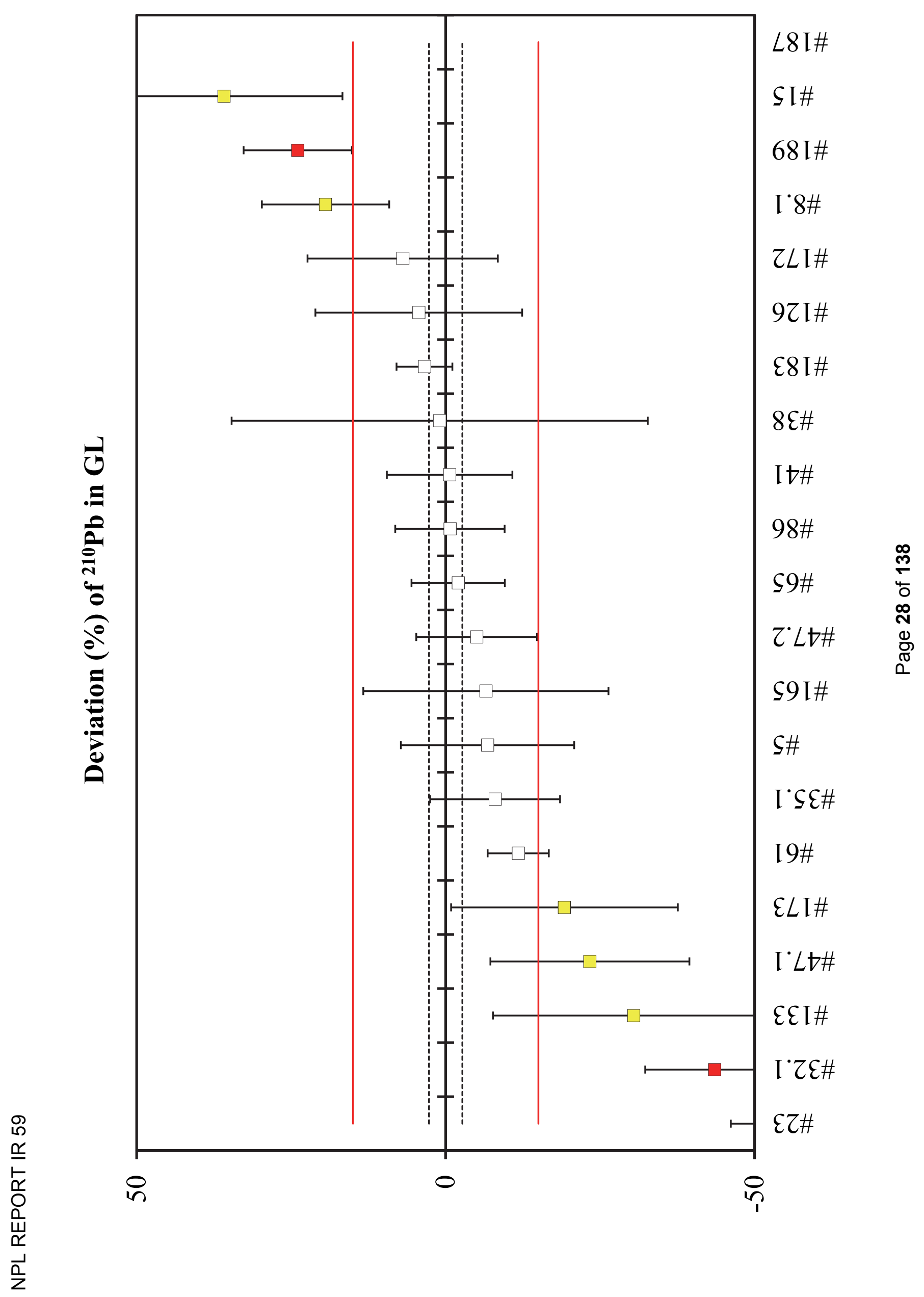




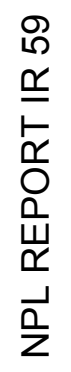

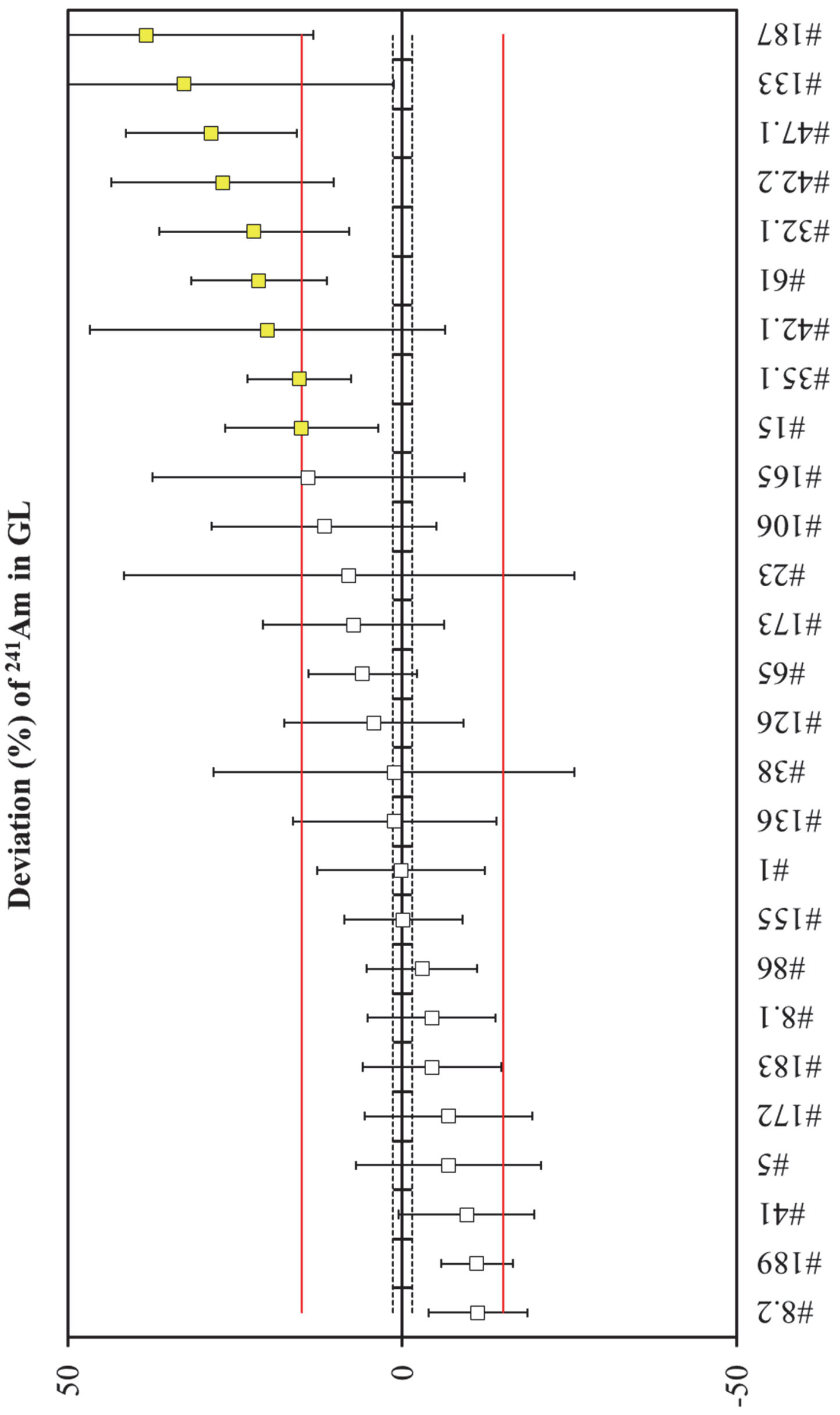




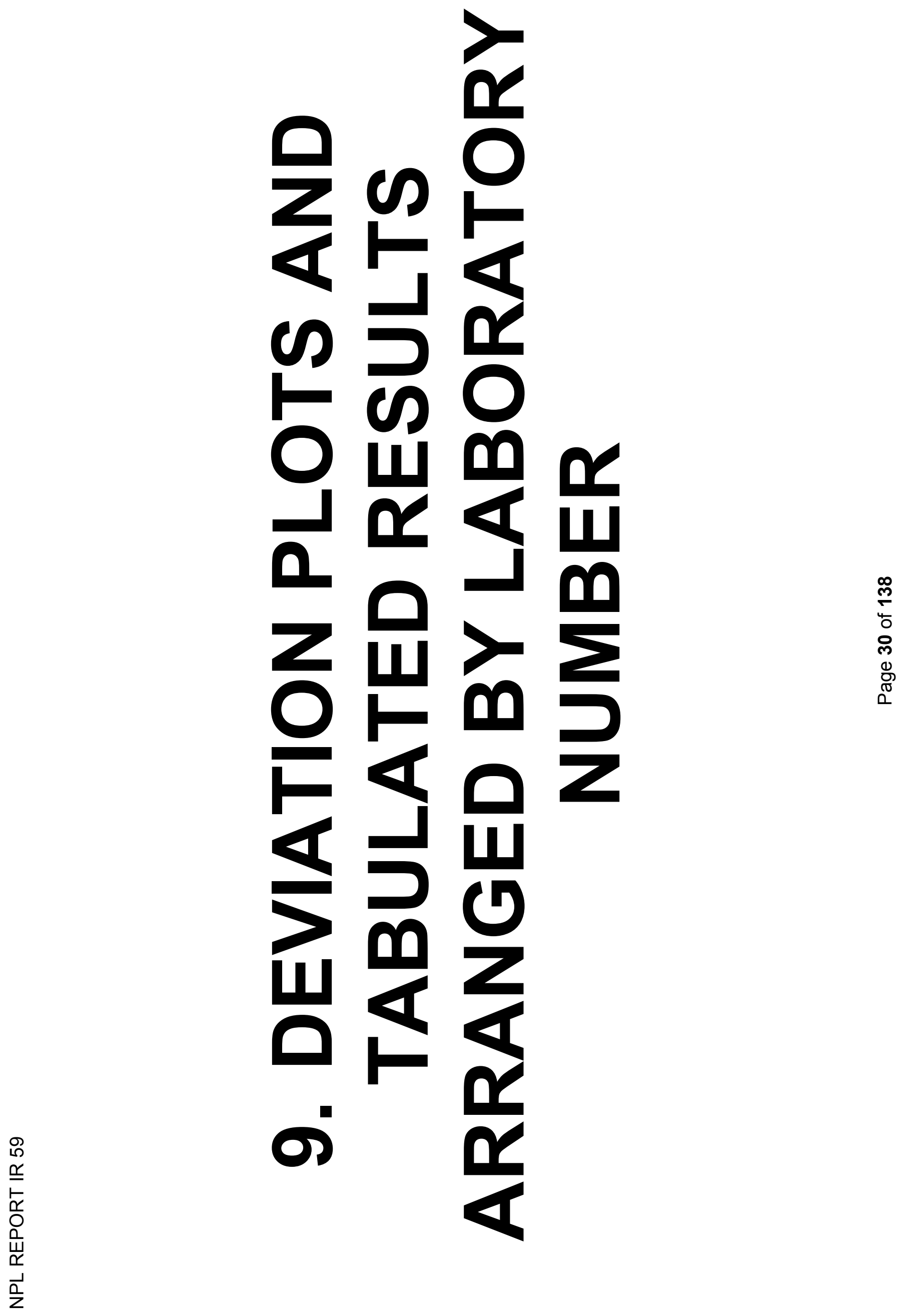


잉

응 ำ

잉. 잉

分

즁

중

ธ

늠 음

웡

을 응

층 응

क ㄷำ

30

$\frac{\pi}{2} \frac{\pi}{2}$

ते

든응 응

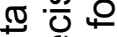

苋 $\frac{1}{2}$

文产

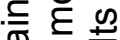

$\stackrel{t}{\square}$

Ð ळ

530

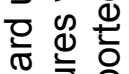

은

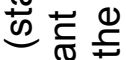

i응

$\checkmark$ 穴 厄

त

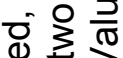

옹

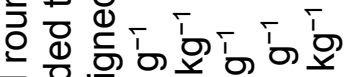

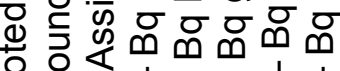

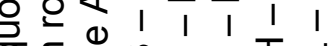

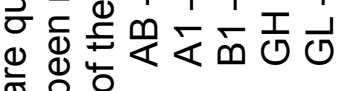

$\infty$
$\stackrel{\infty}{7}$
$\frac{4}{0}$
$\frac{m}{m}$
0
0
0
0 ๙ 00 


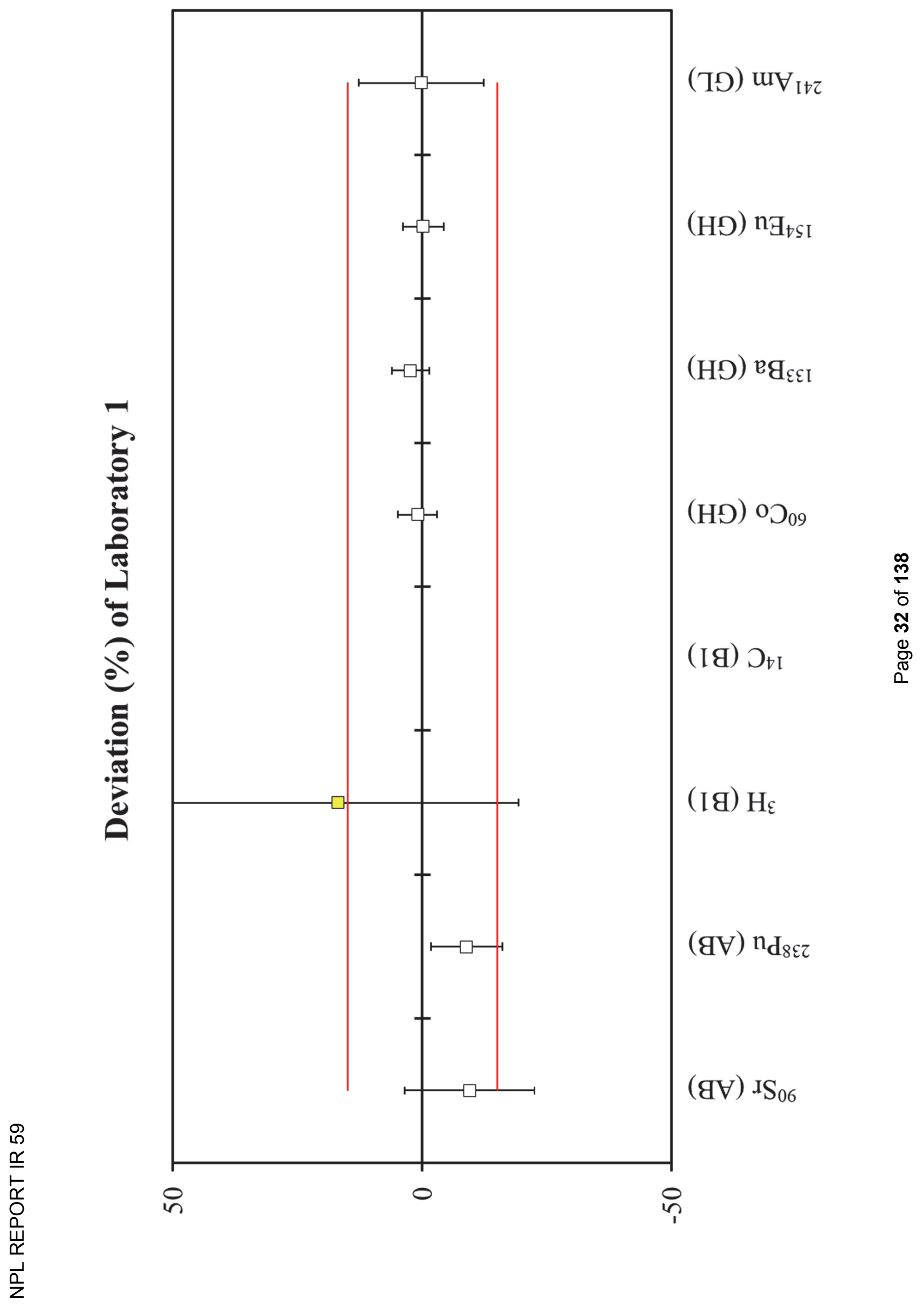




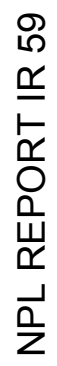

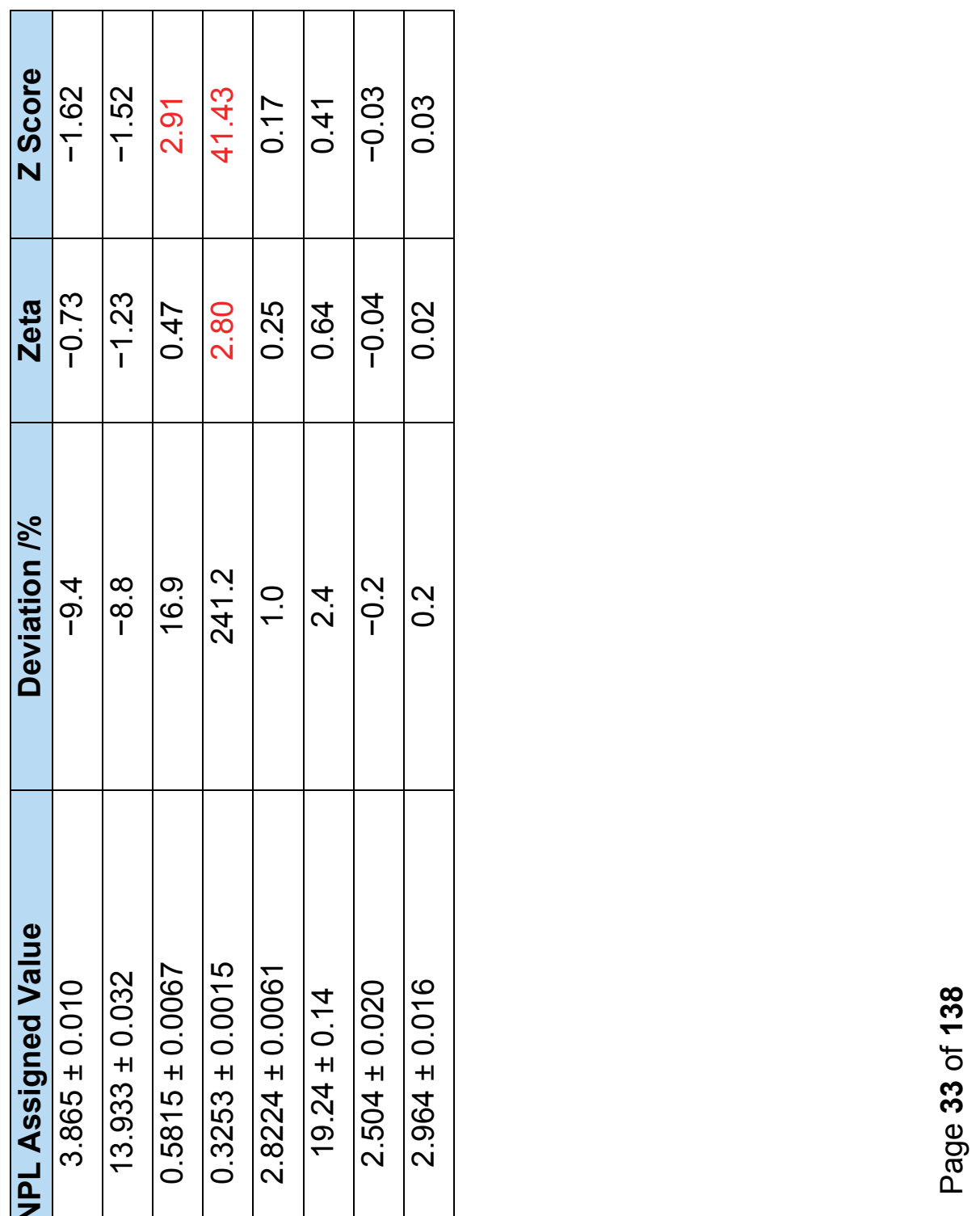

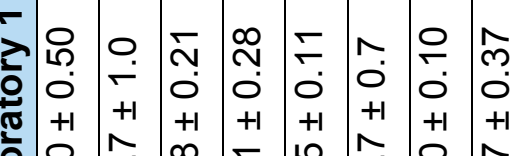

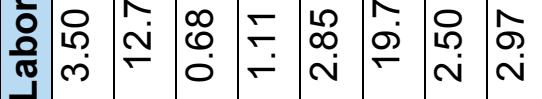

응

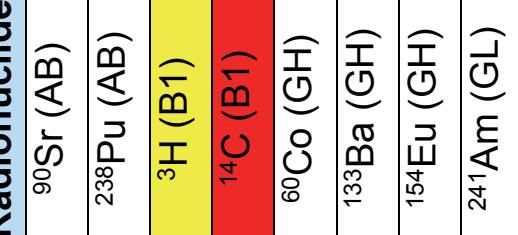




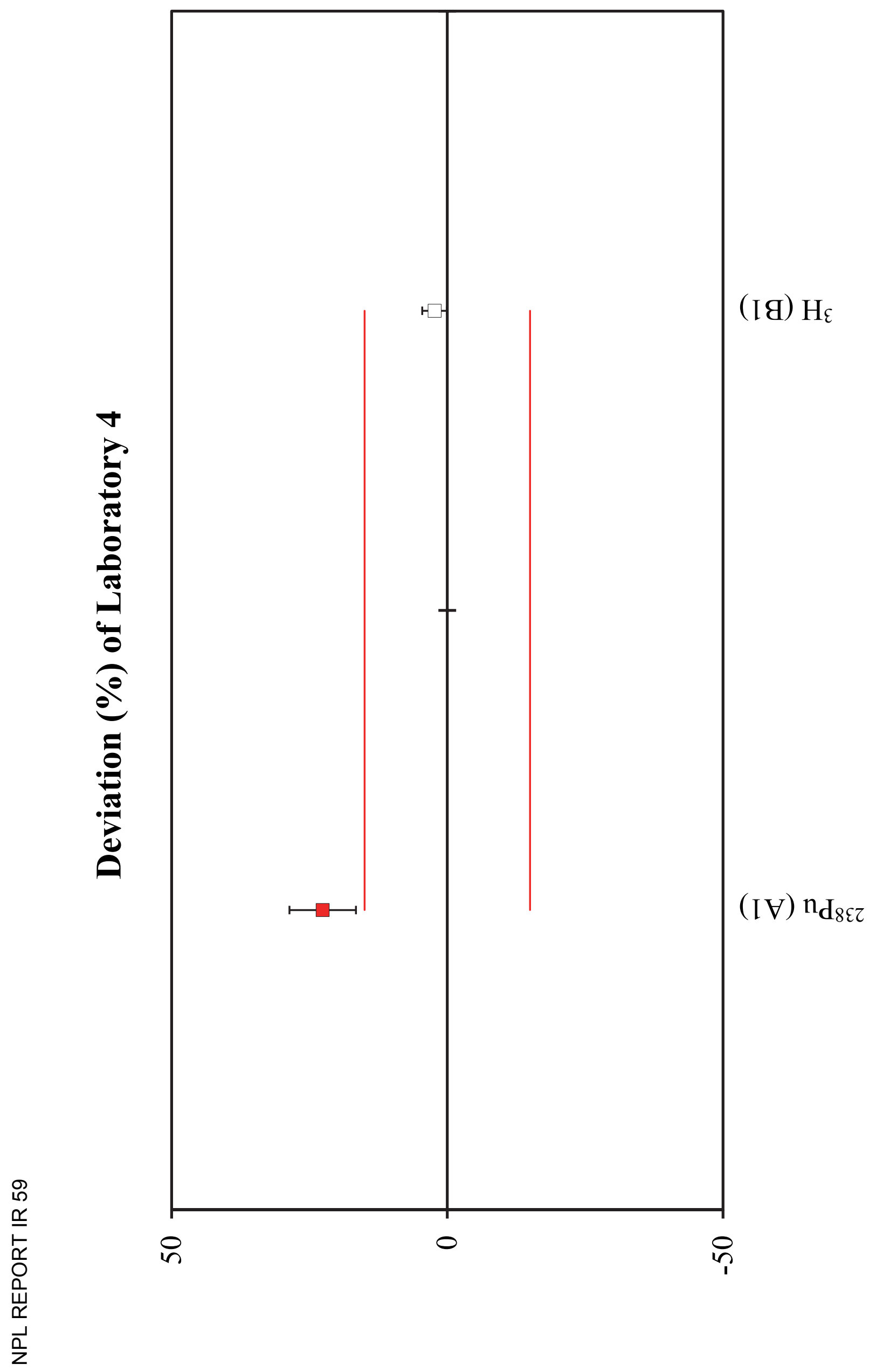

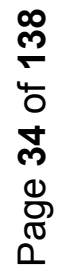




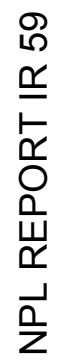

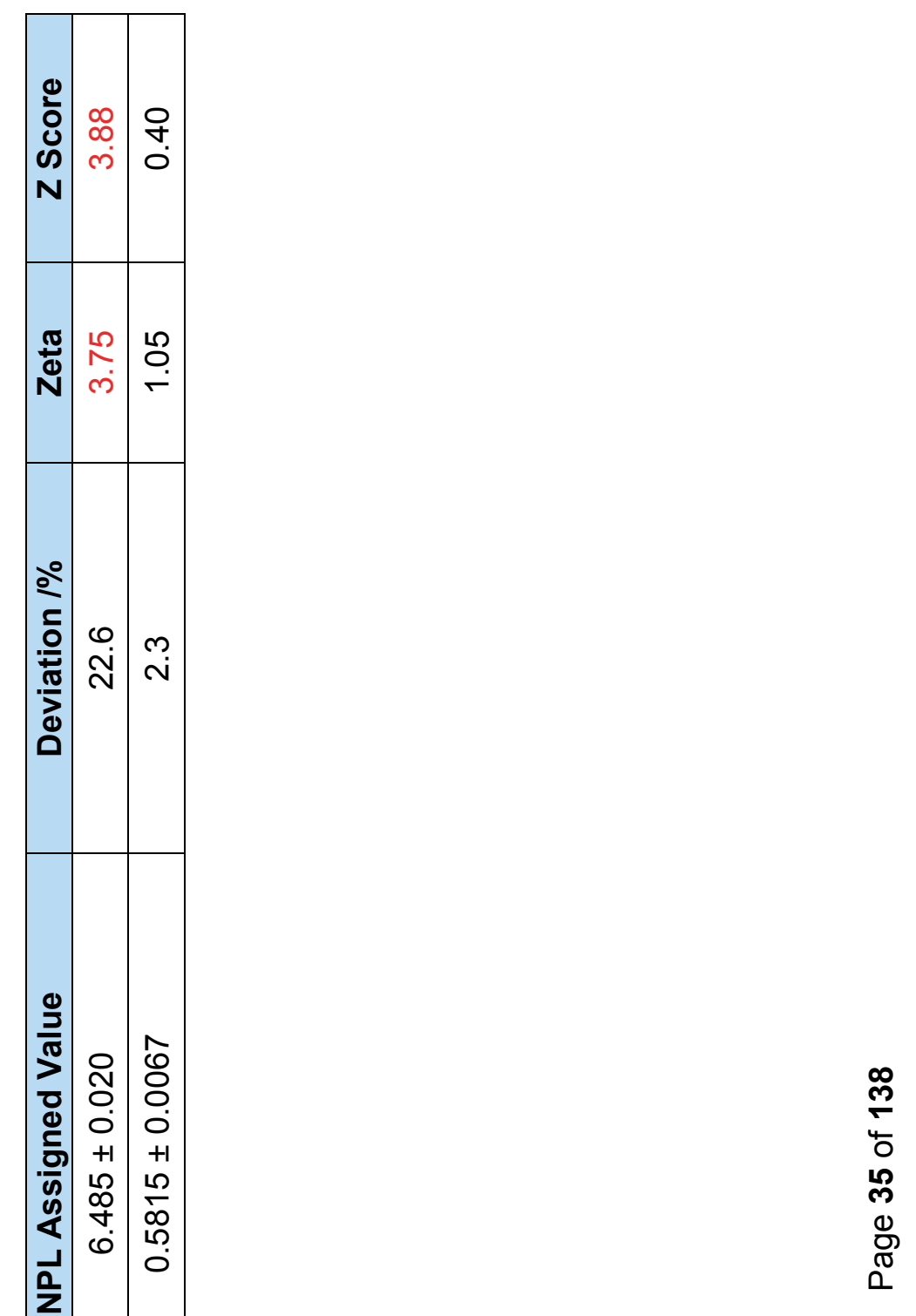

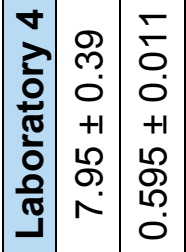

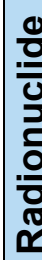

$\widehat{\tilde{\sigma}}$ 


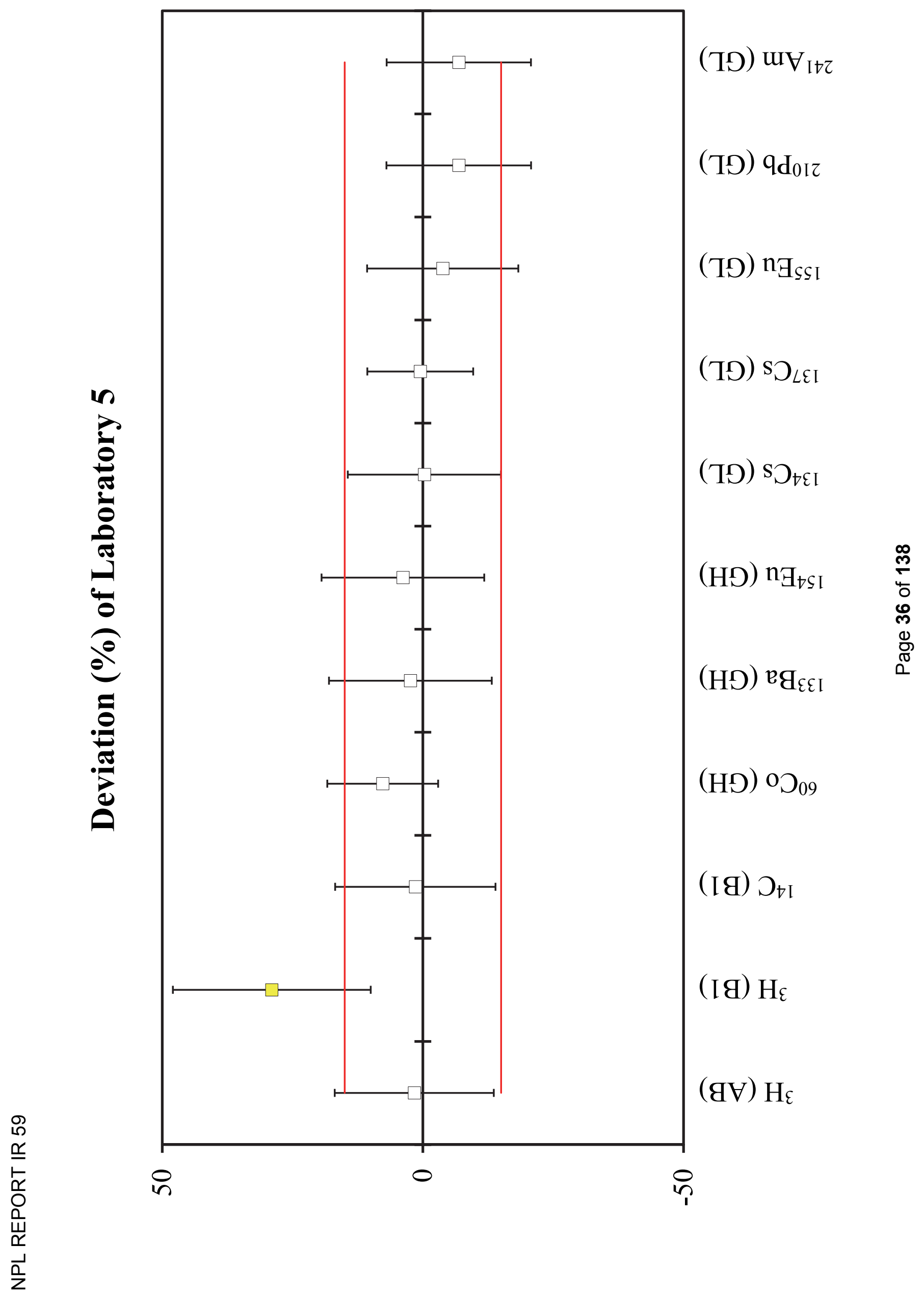




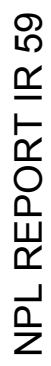

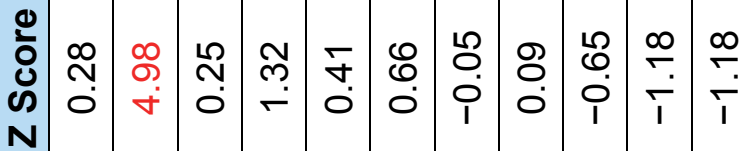

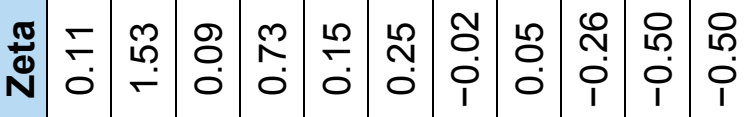

2

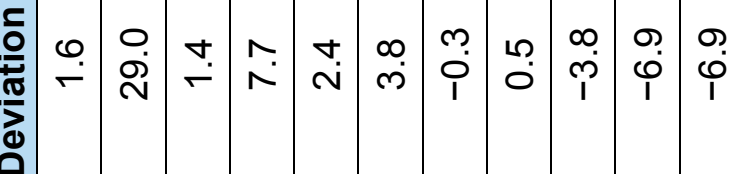

$\frac{\sqrt{2}}{\frac{2}{2}}$

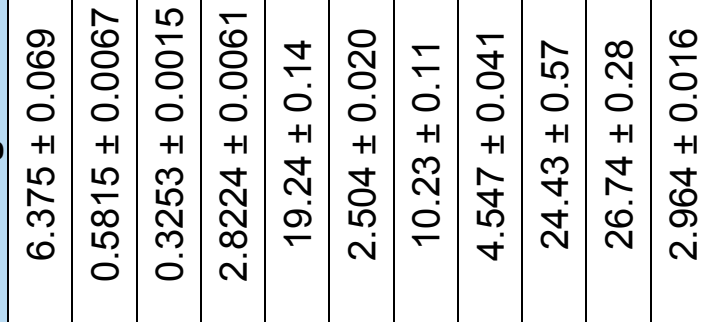

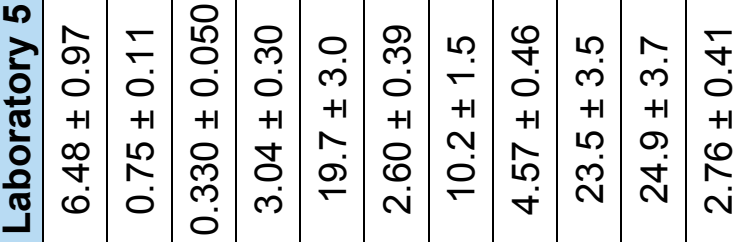

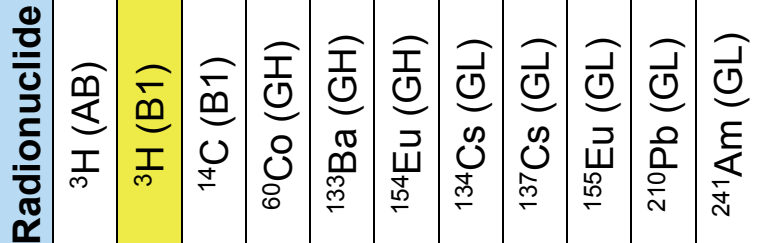




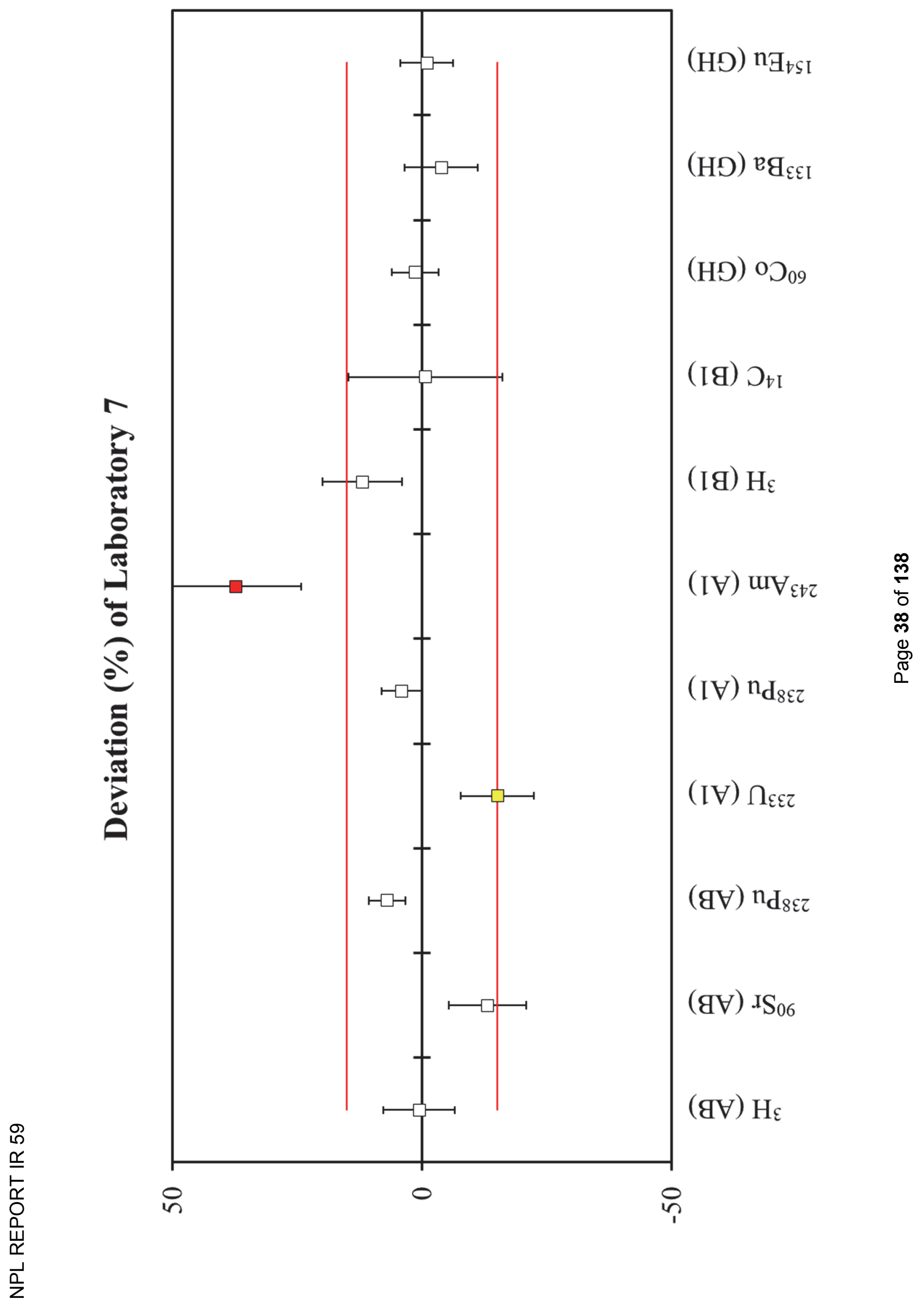




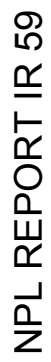

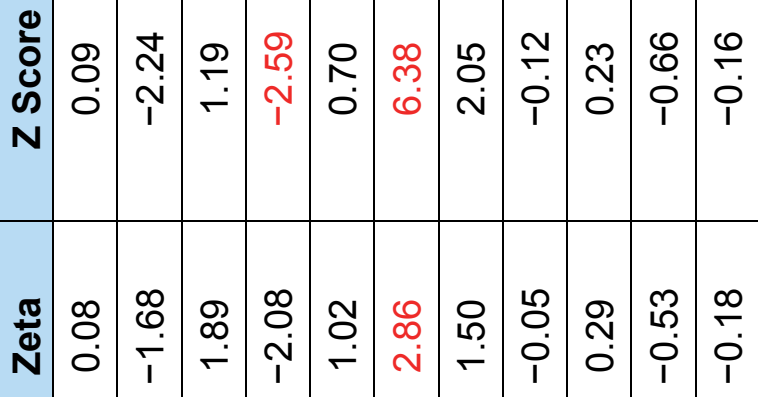

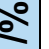

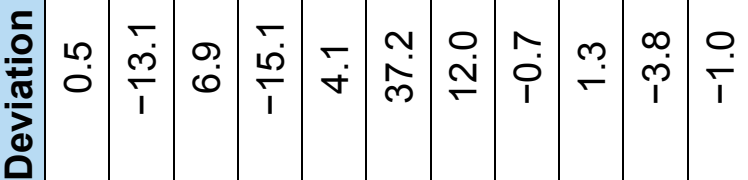

$\frac{0}{\frac{1}{2}}$

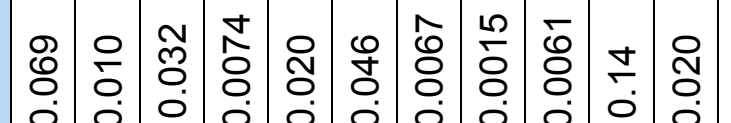

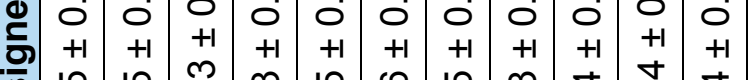

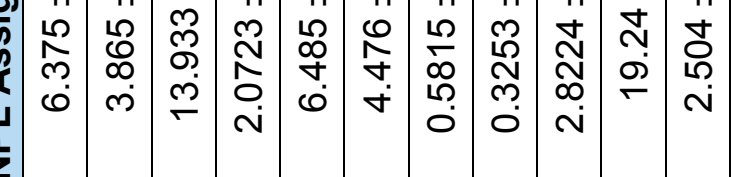

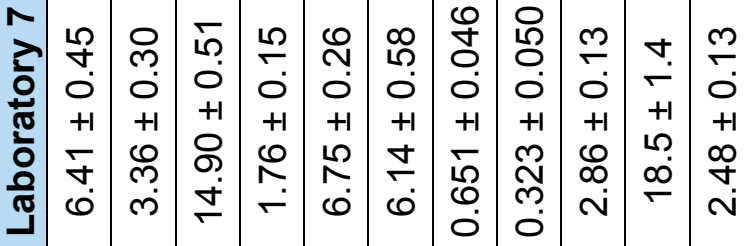

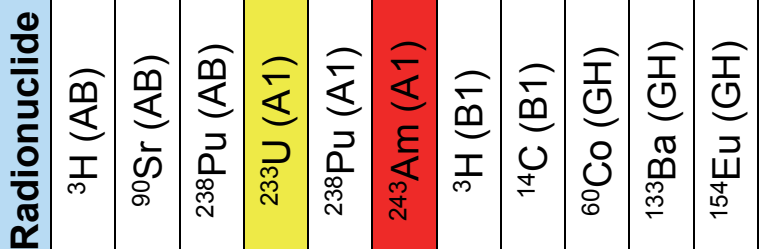

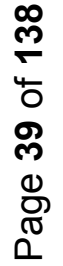




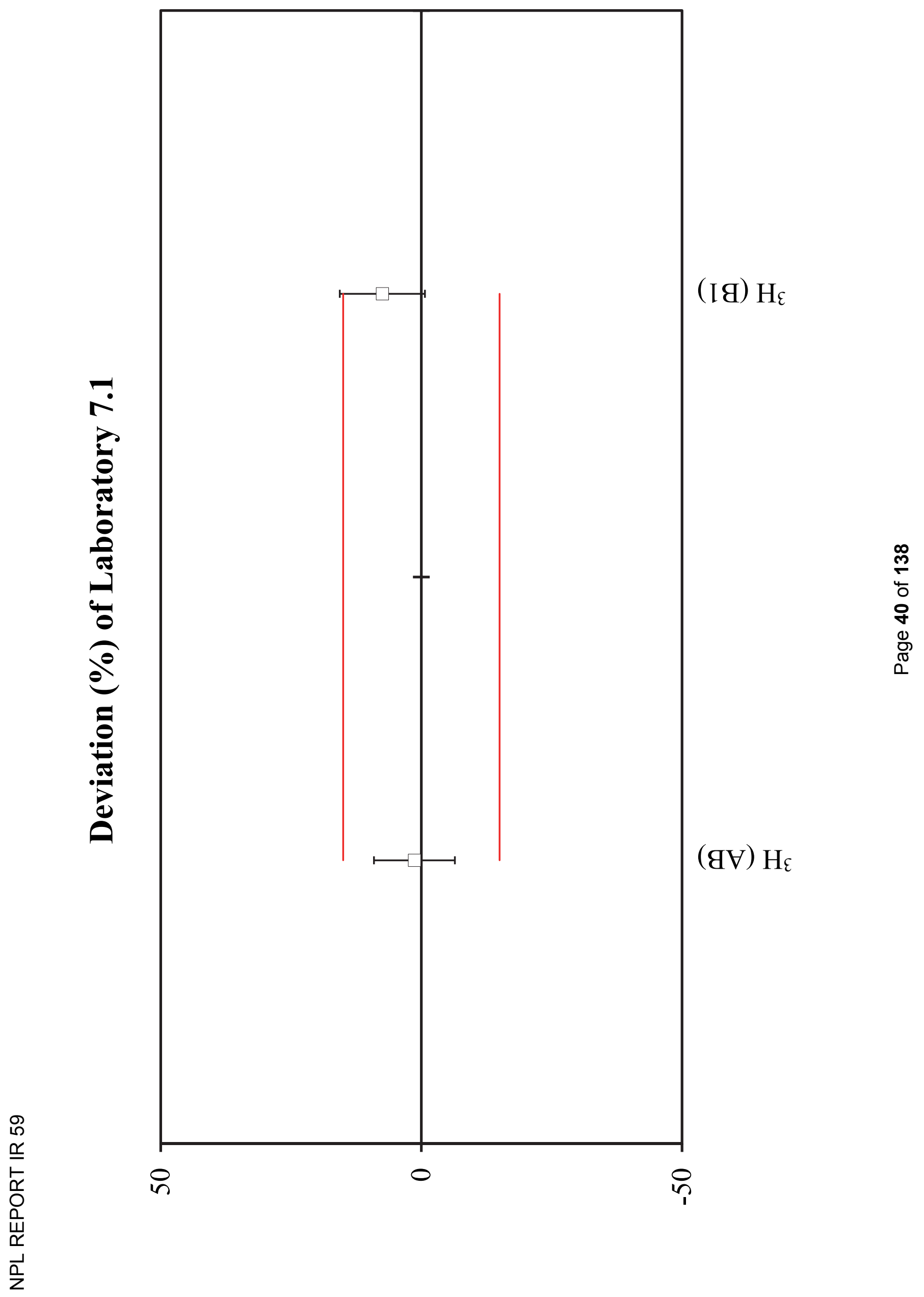




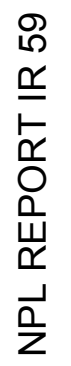

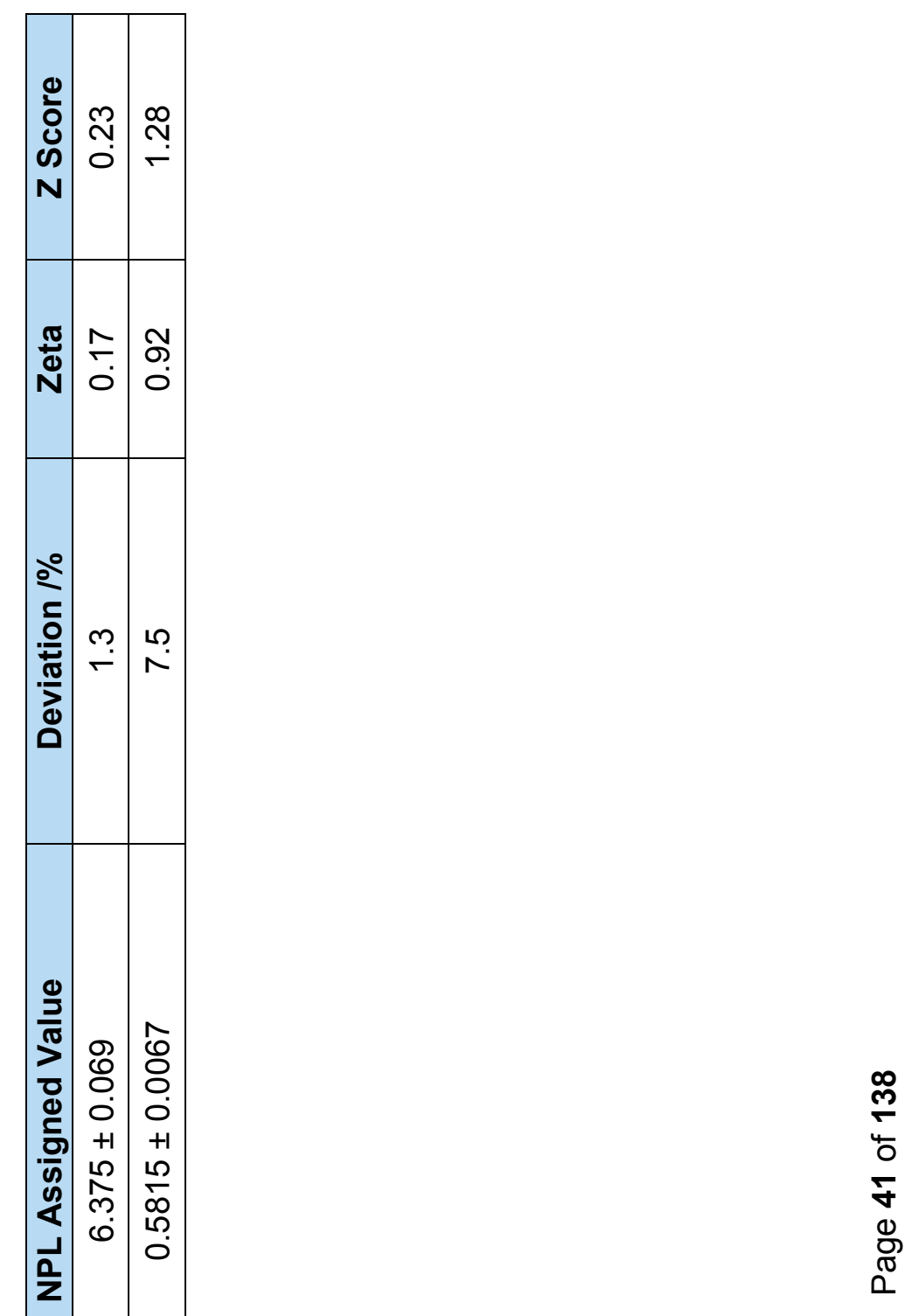

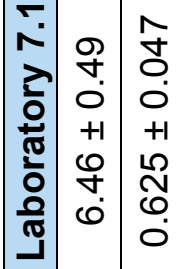

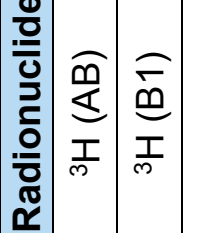




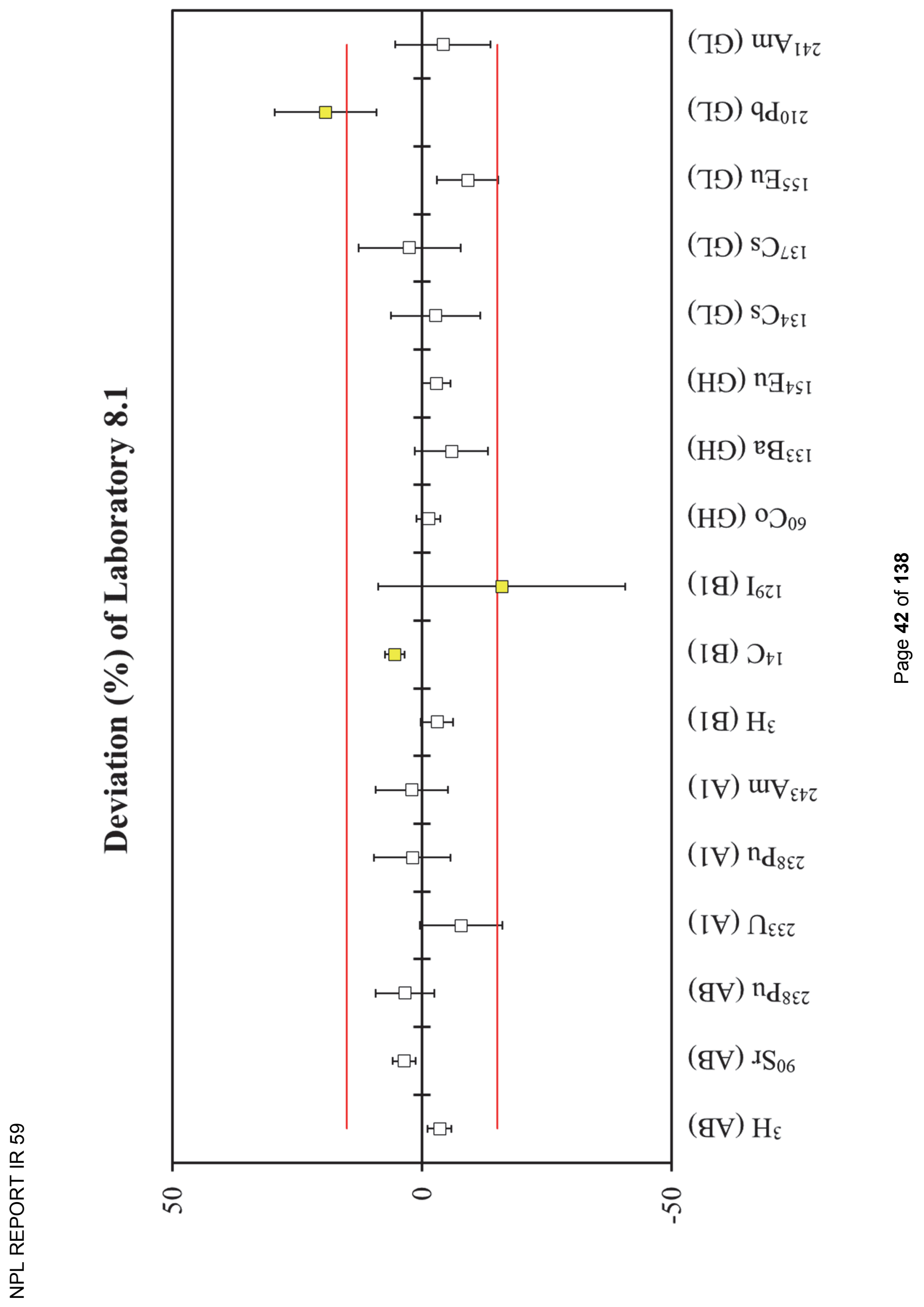




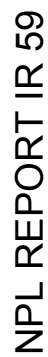

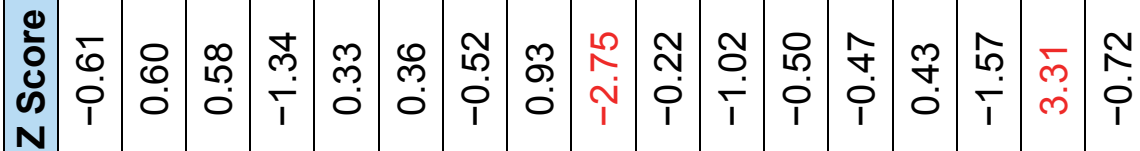

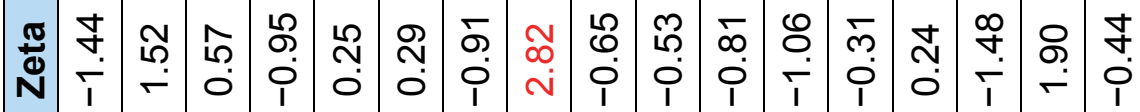

$\stackrel{0}{\circ}$

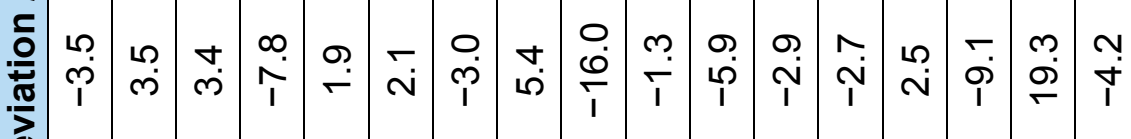

อั

$\frac{0}{\frac{\pi}{\pi}}$

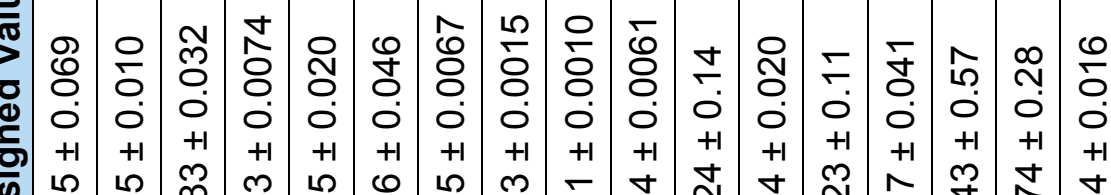

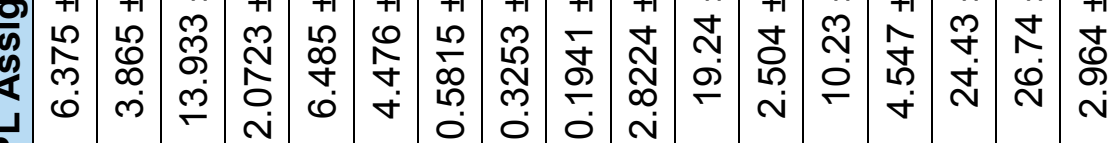

$\underline{\mathbf{n}}$

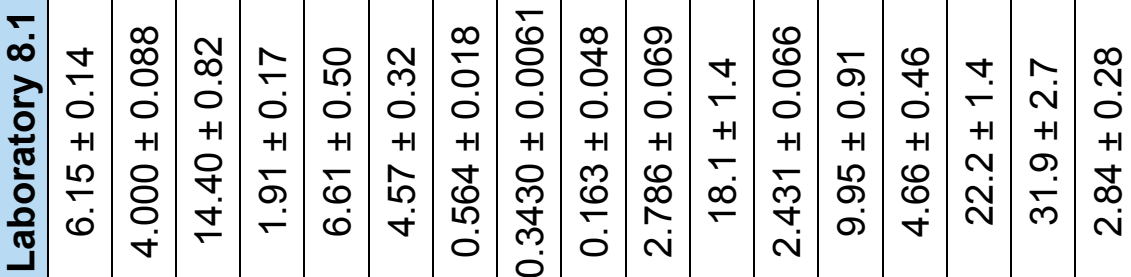

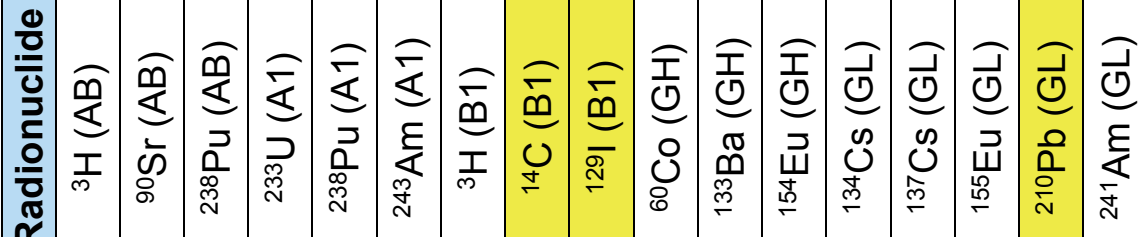

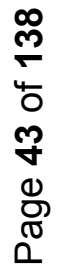




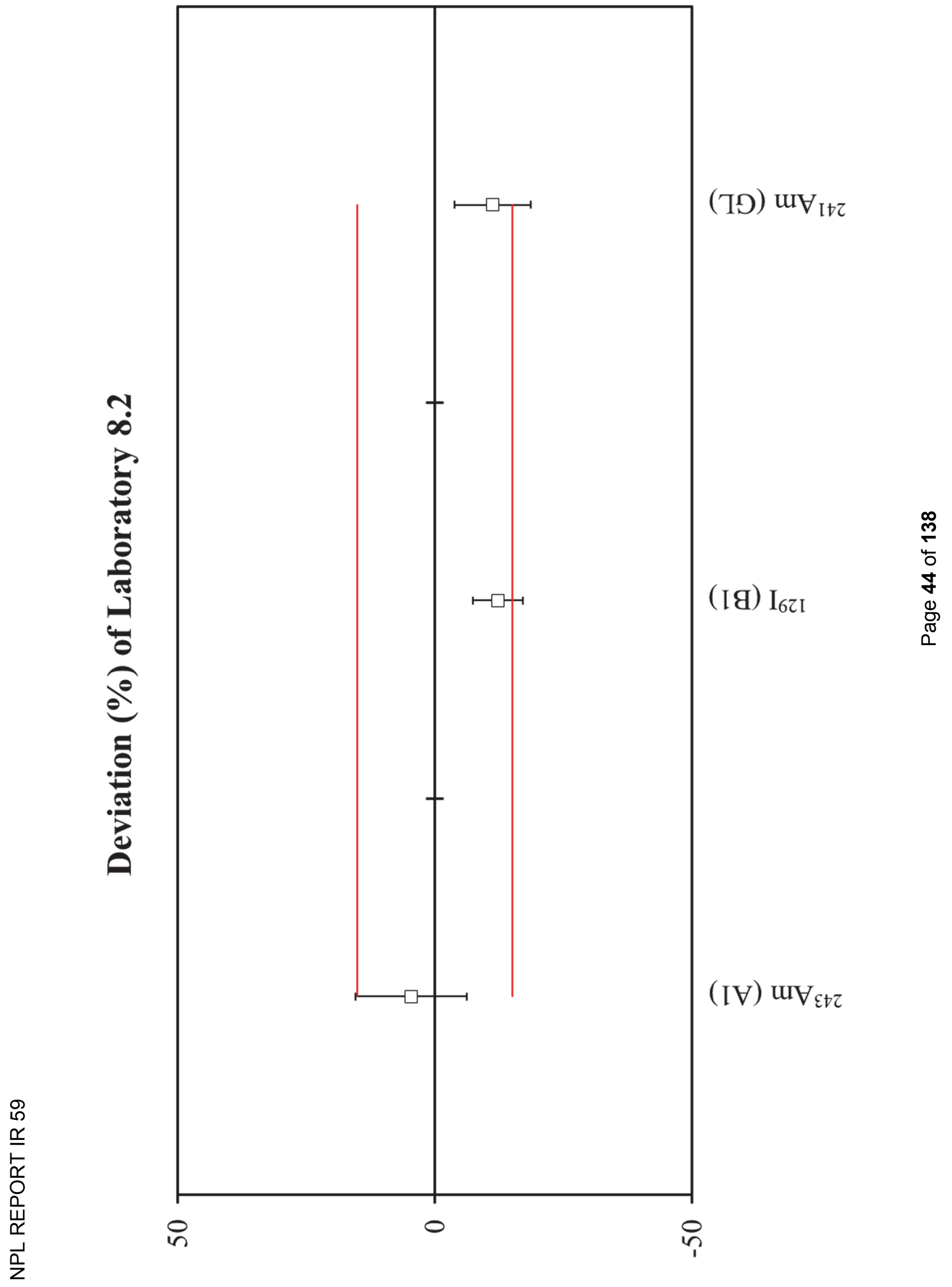




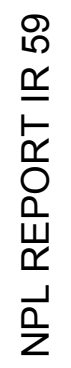

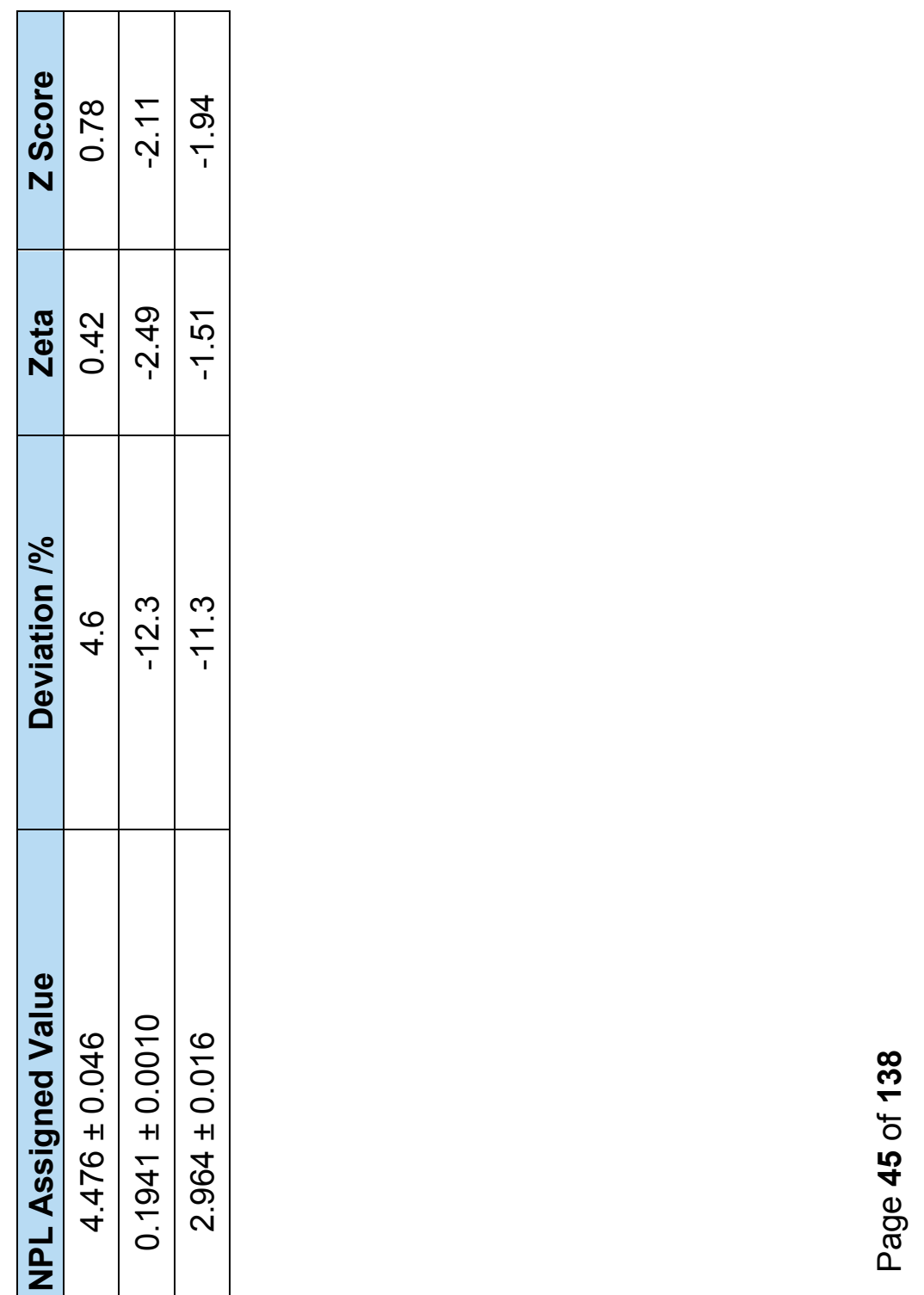

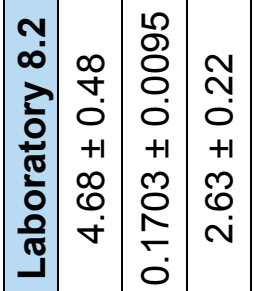

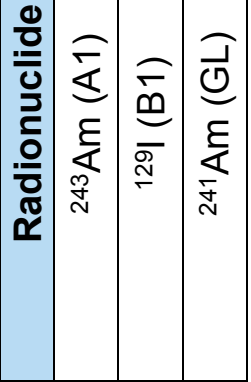




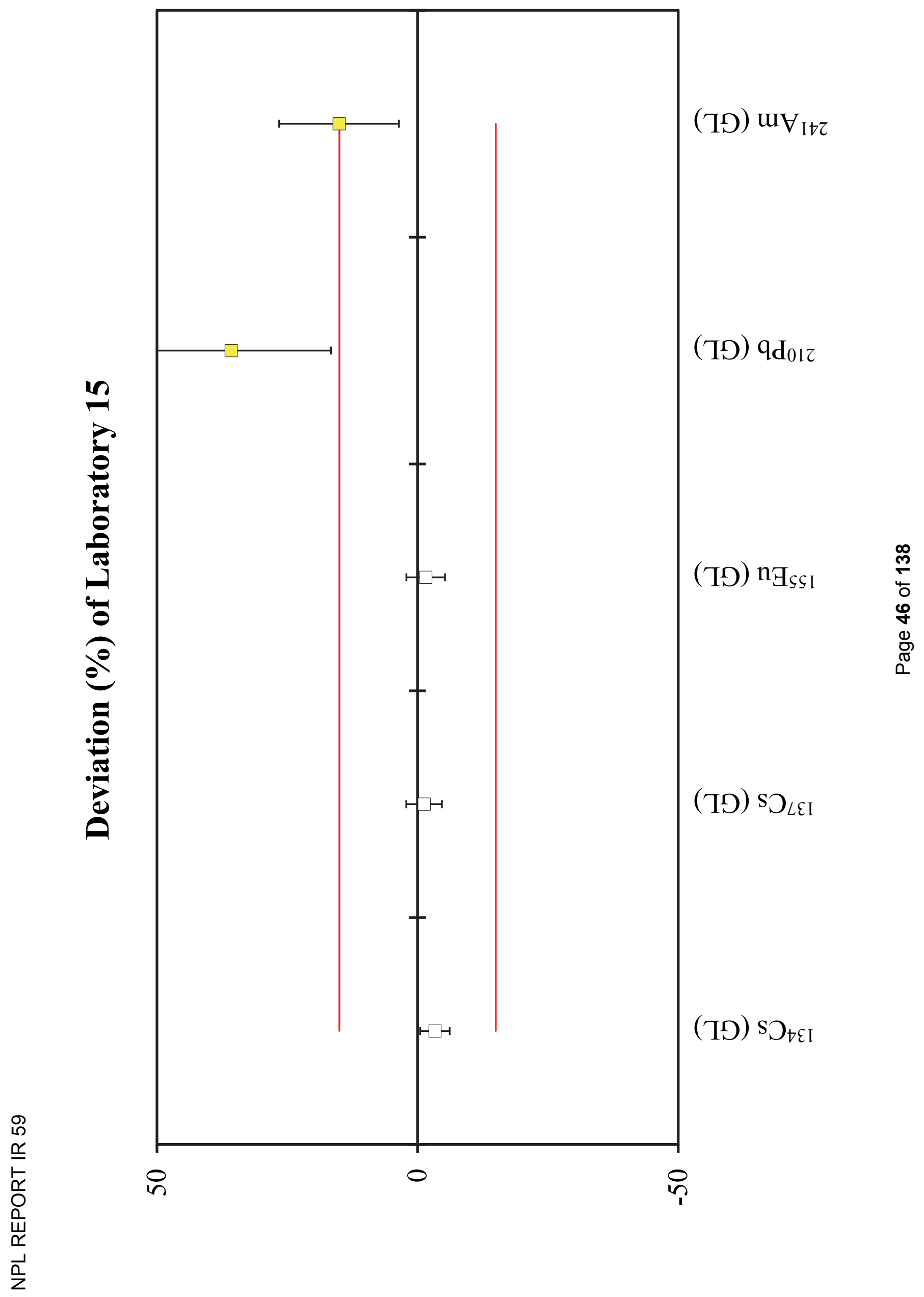




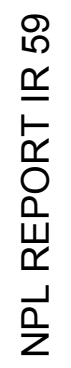

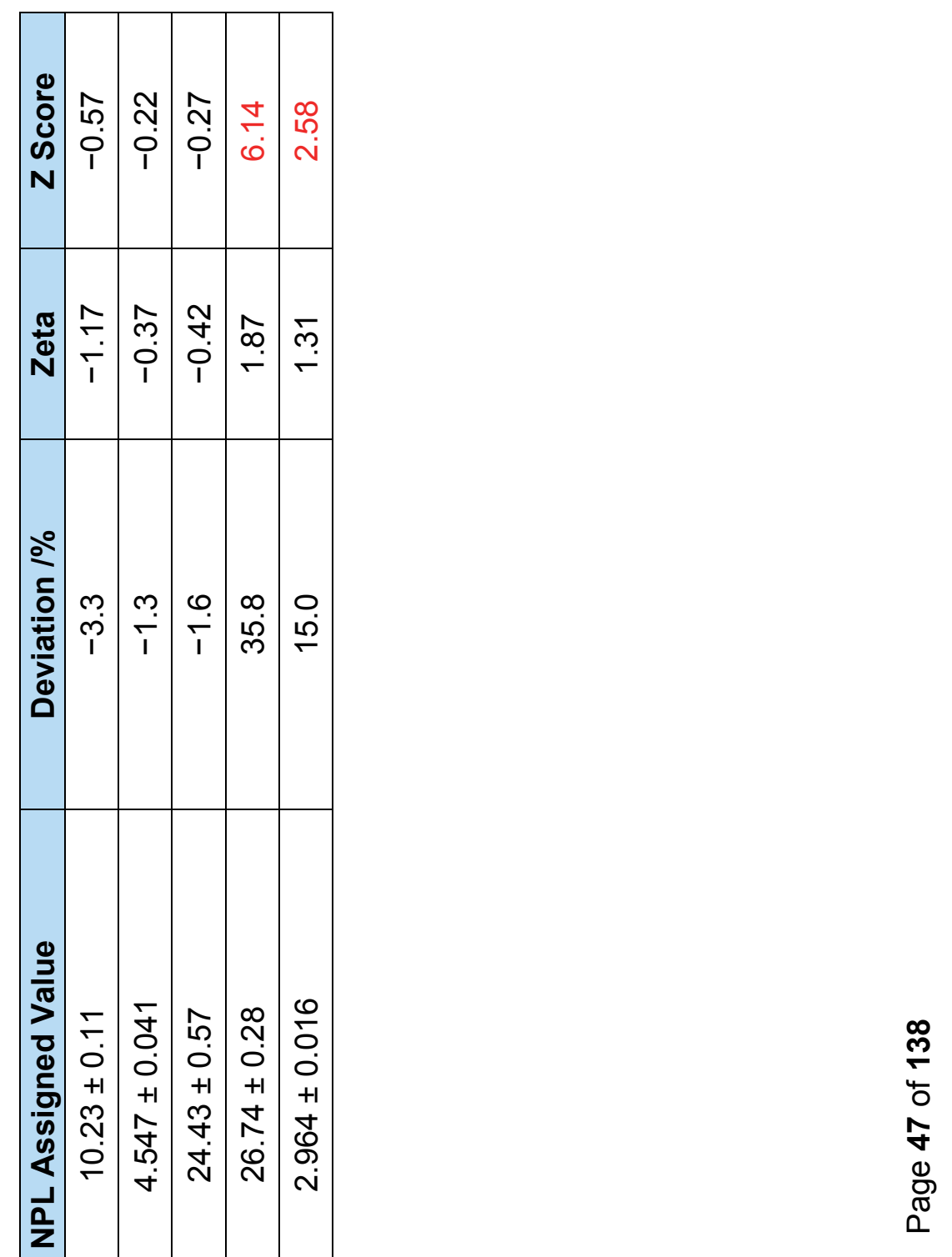

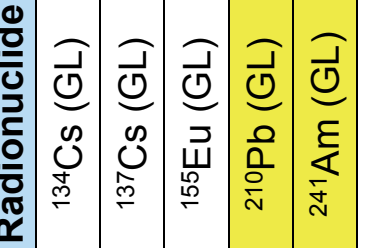




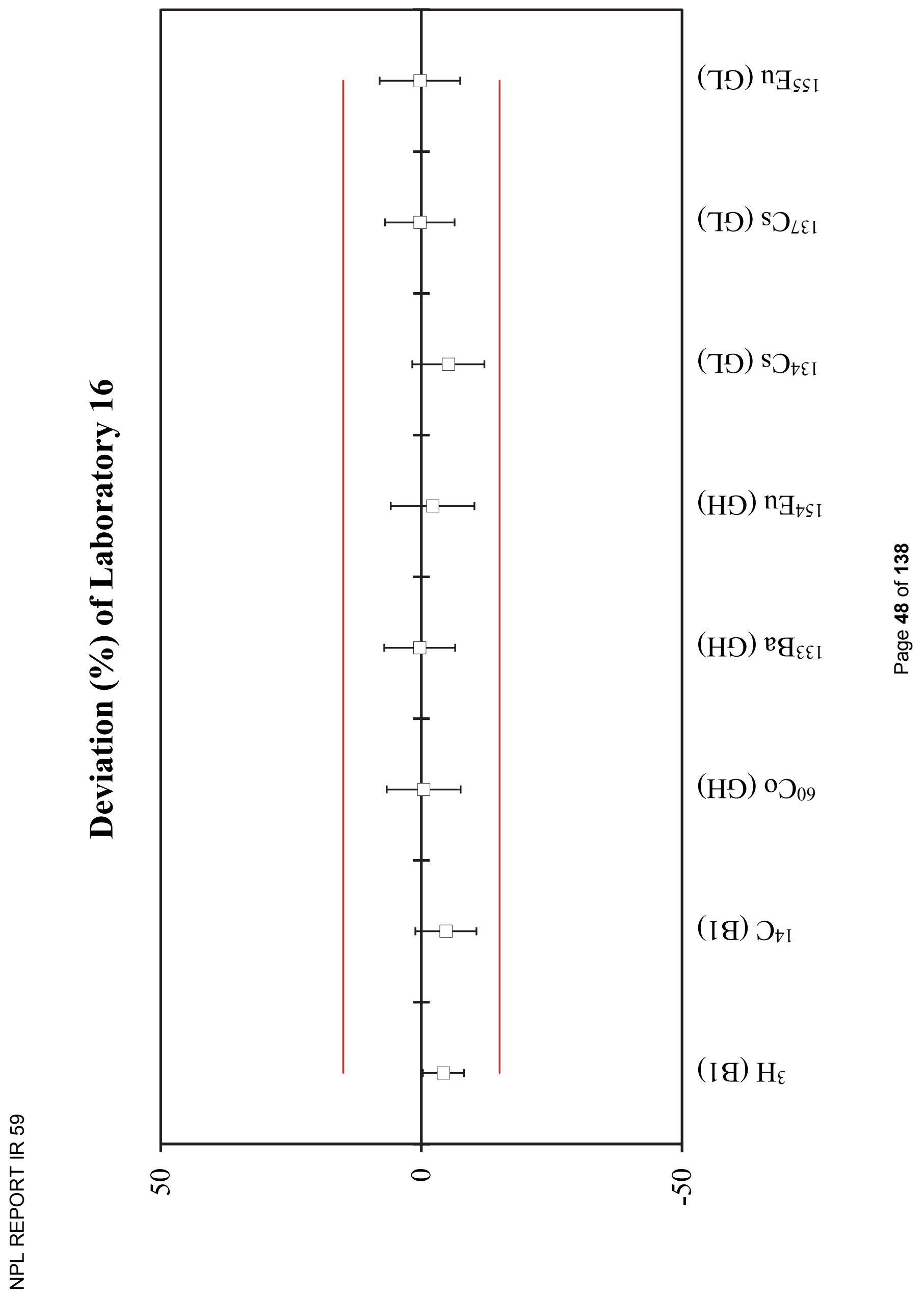




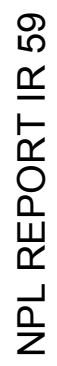

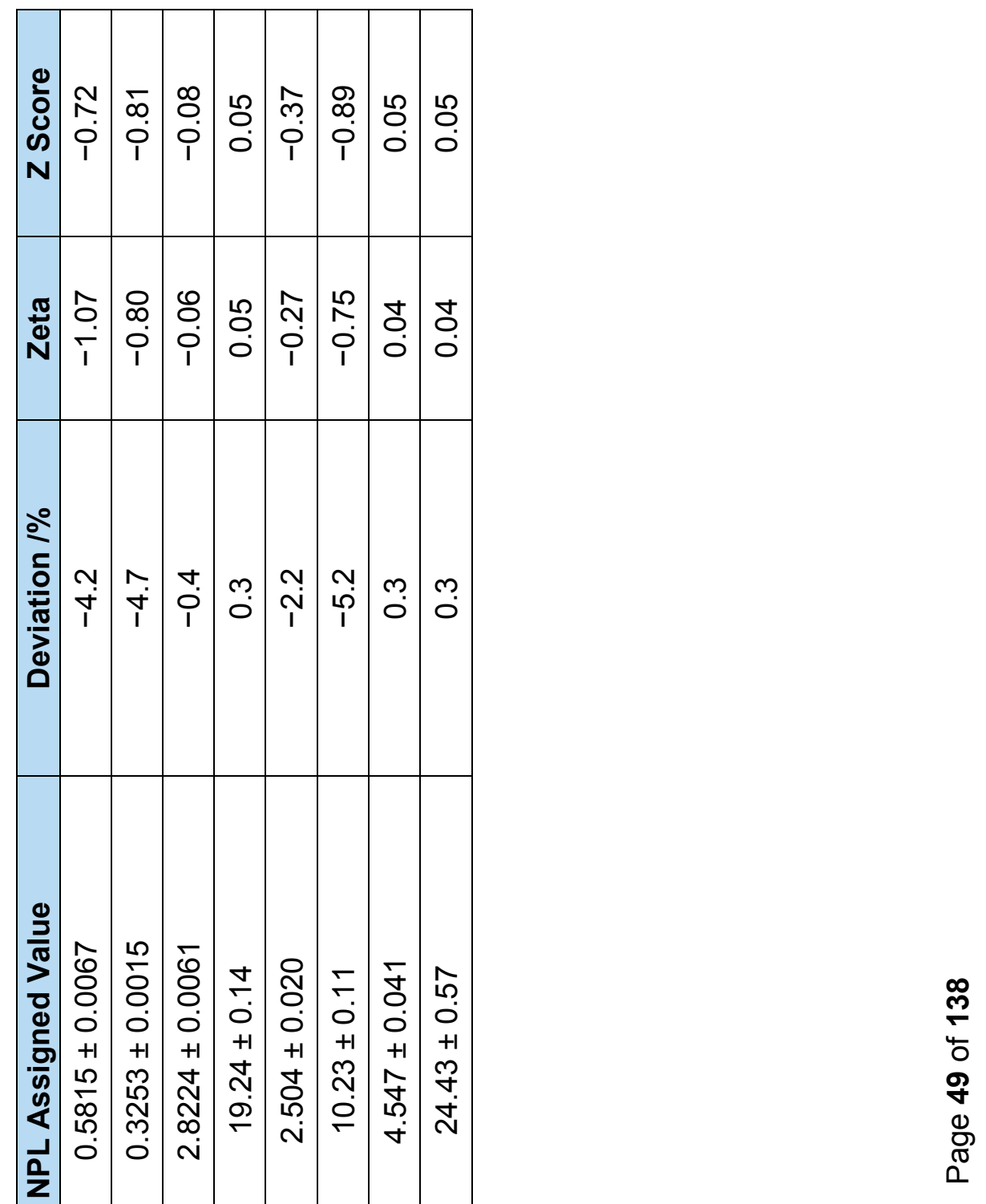

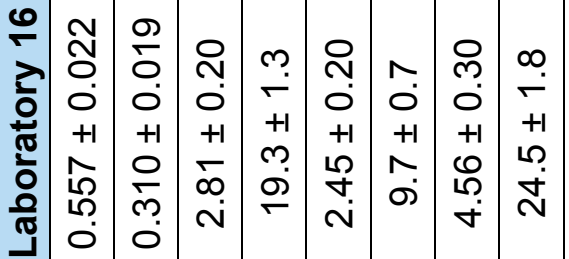

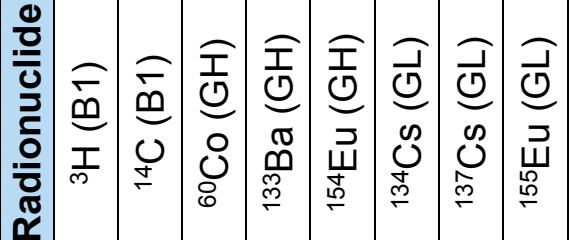




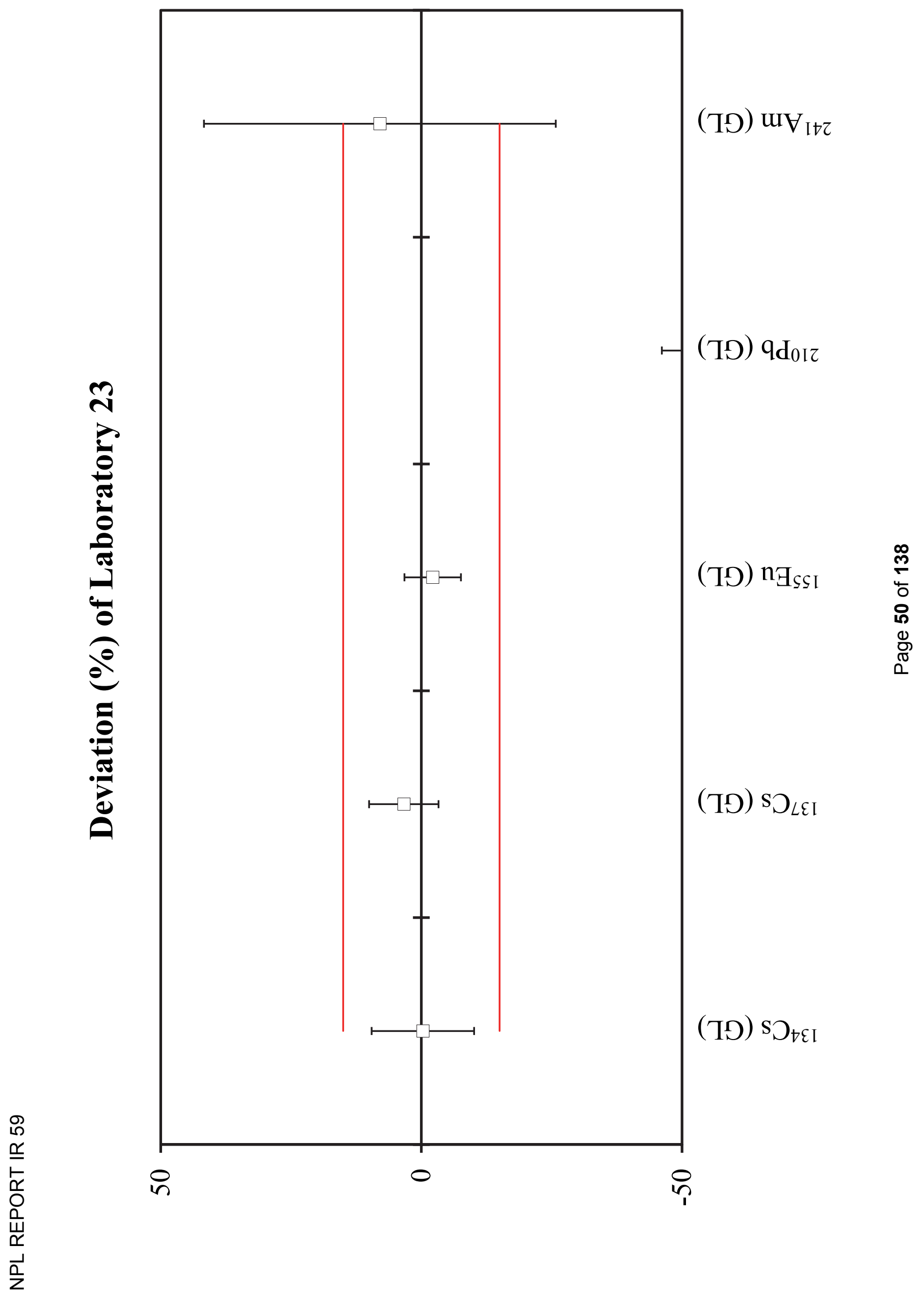




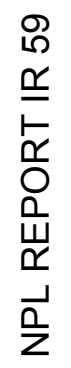

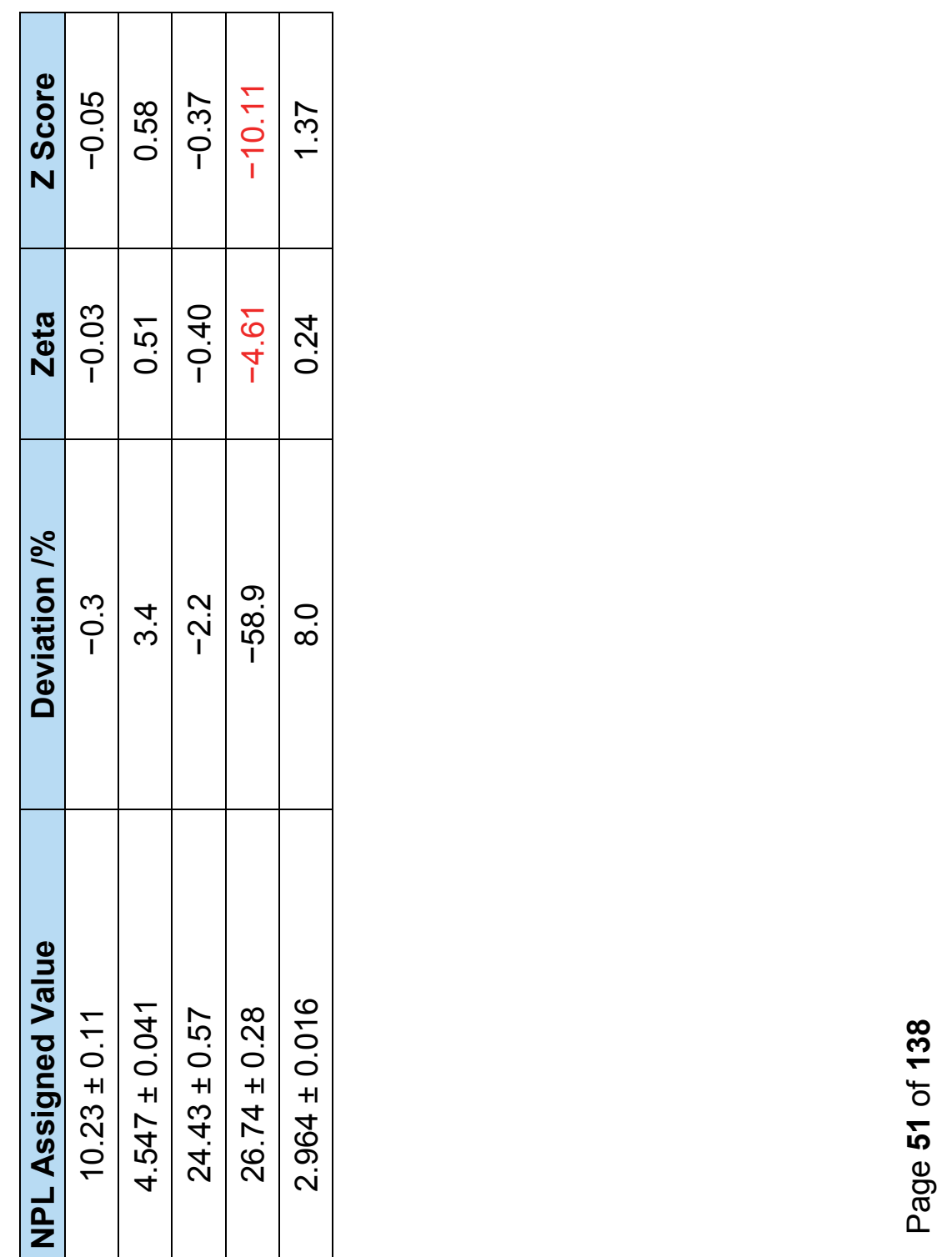

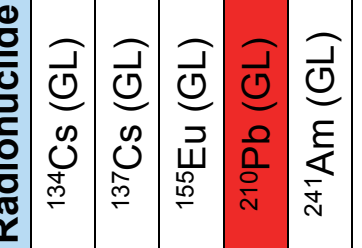




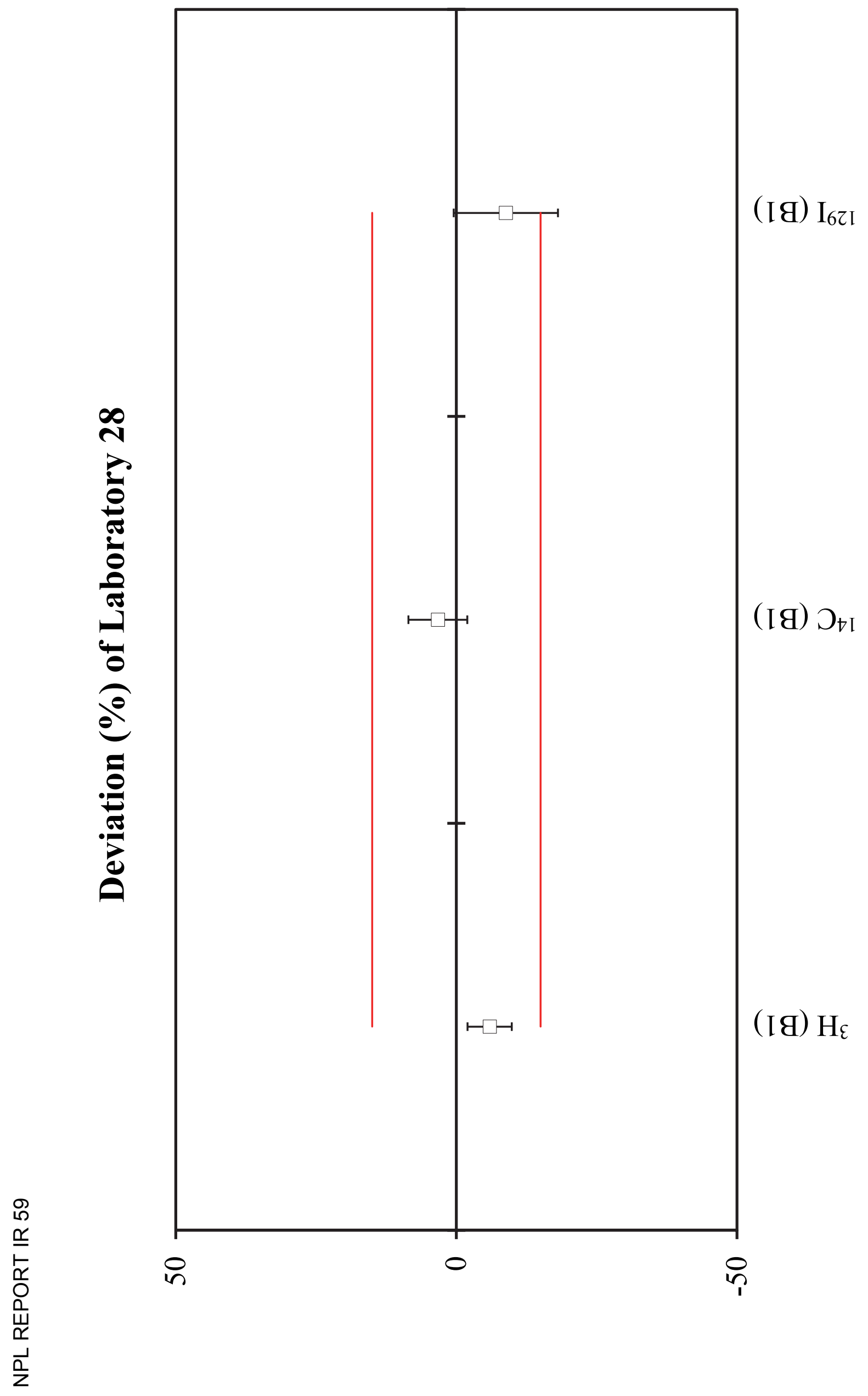

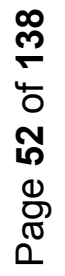




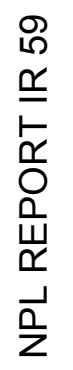

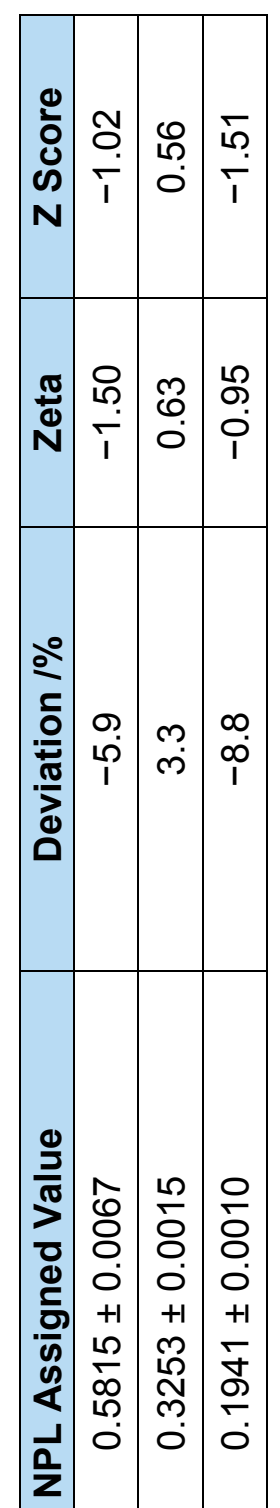

$\infty$
$\stackrel{9}{\leftarrow}$
0
0
0
0
0
0
0

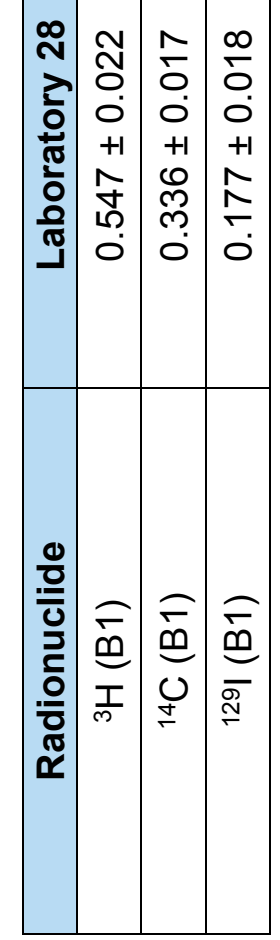




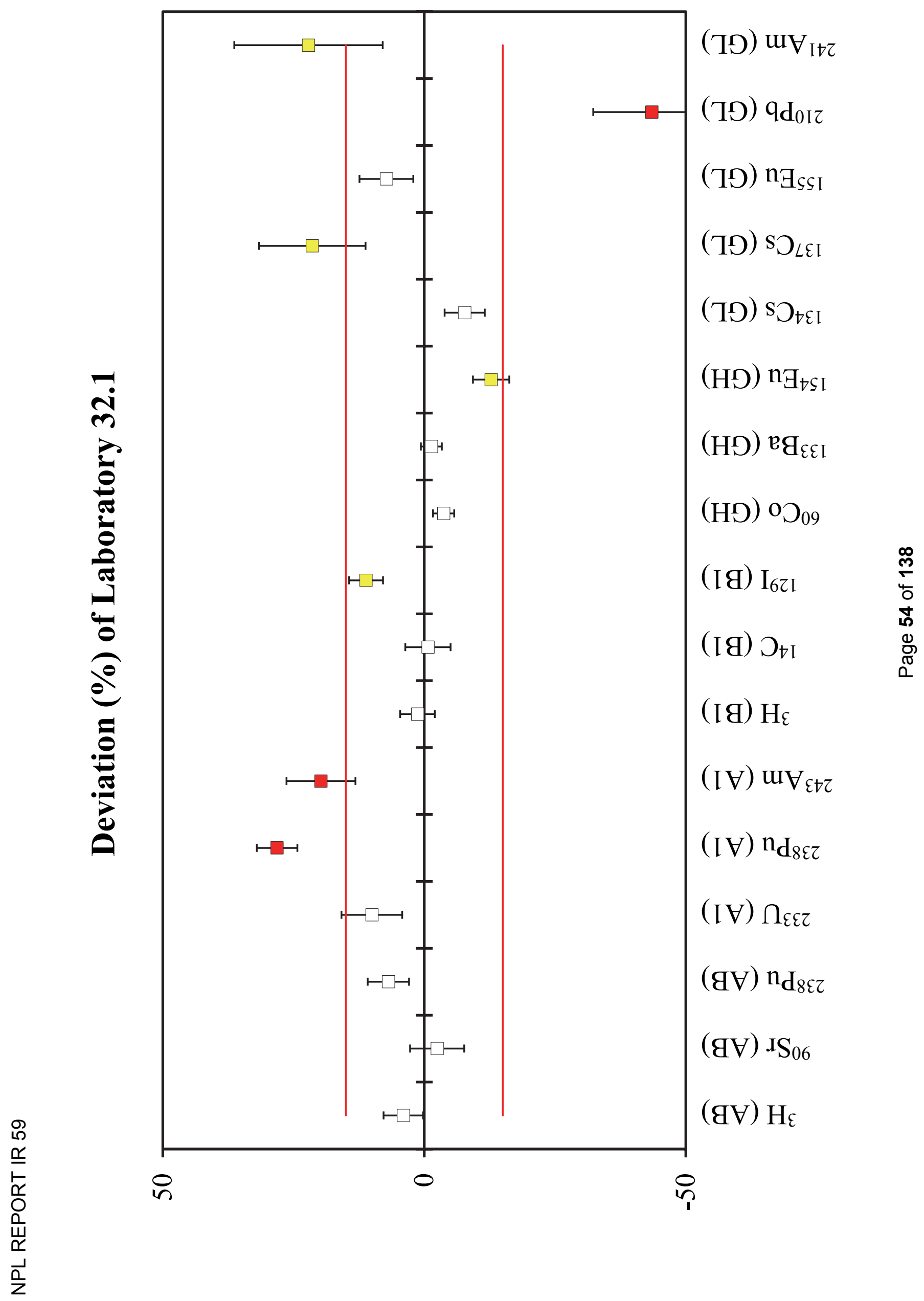




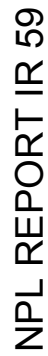

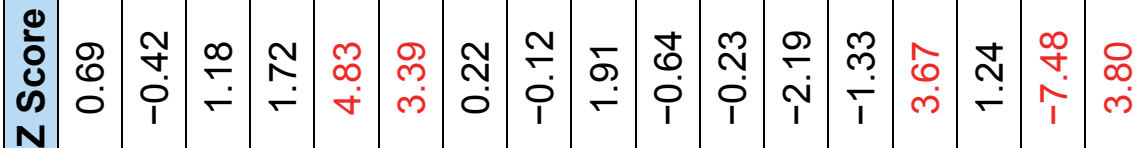

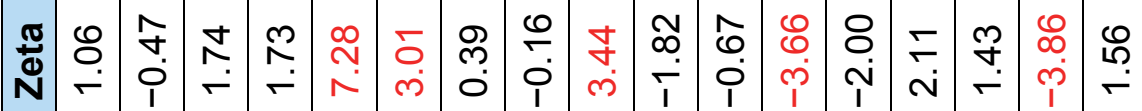

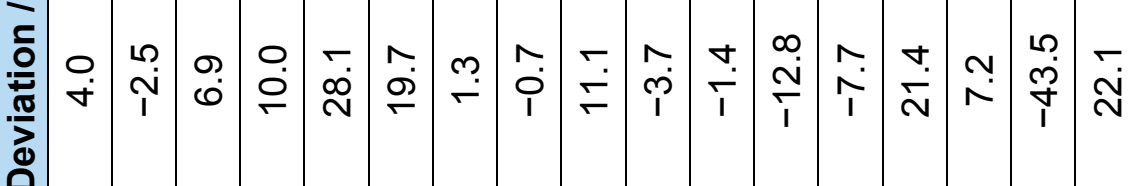

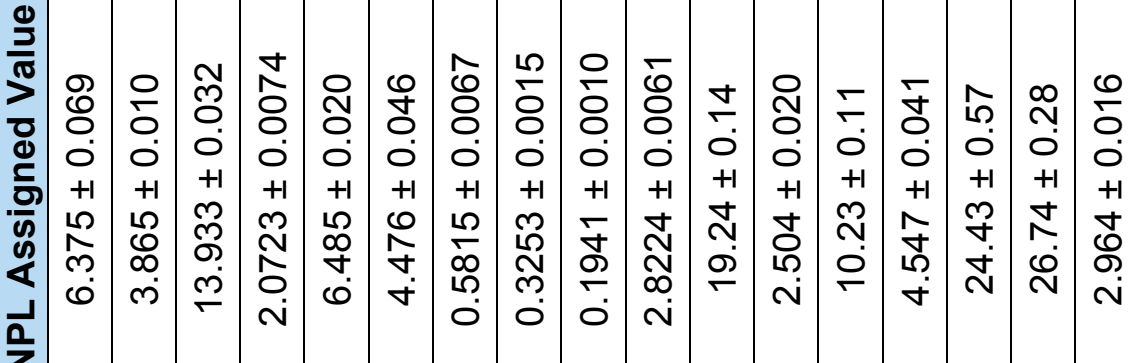

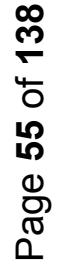

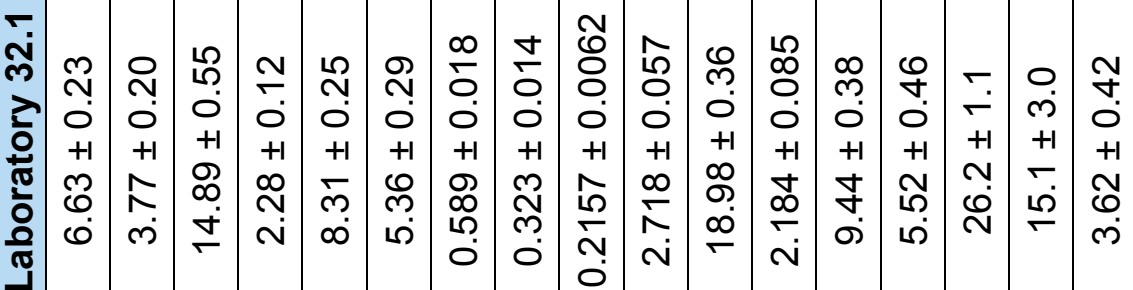

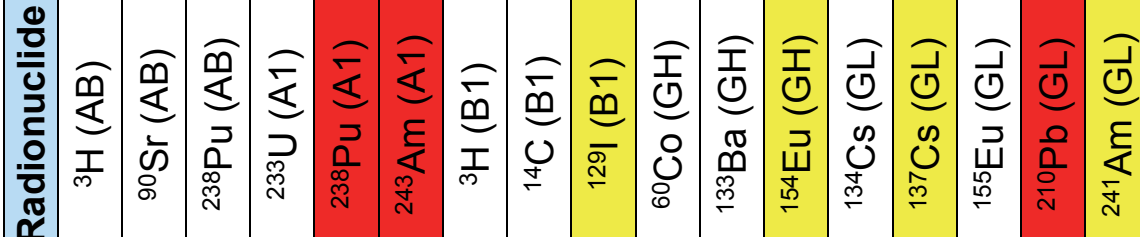




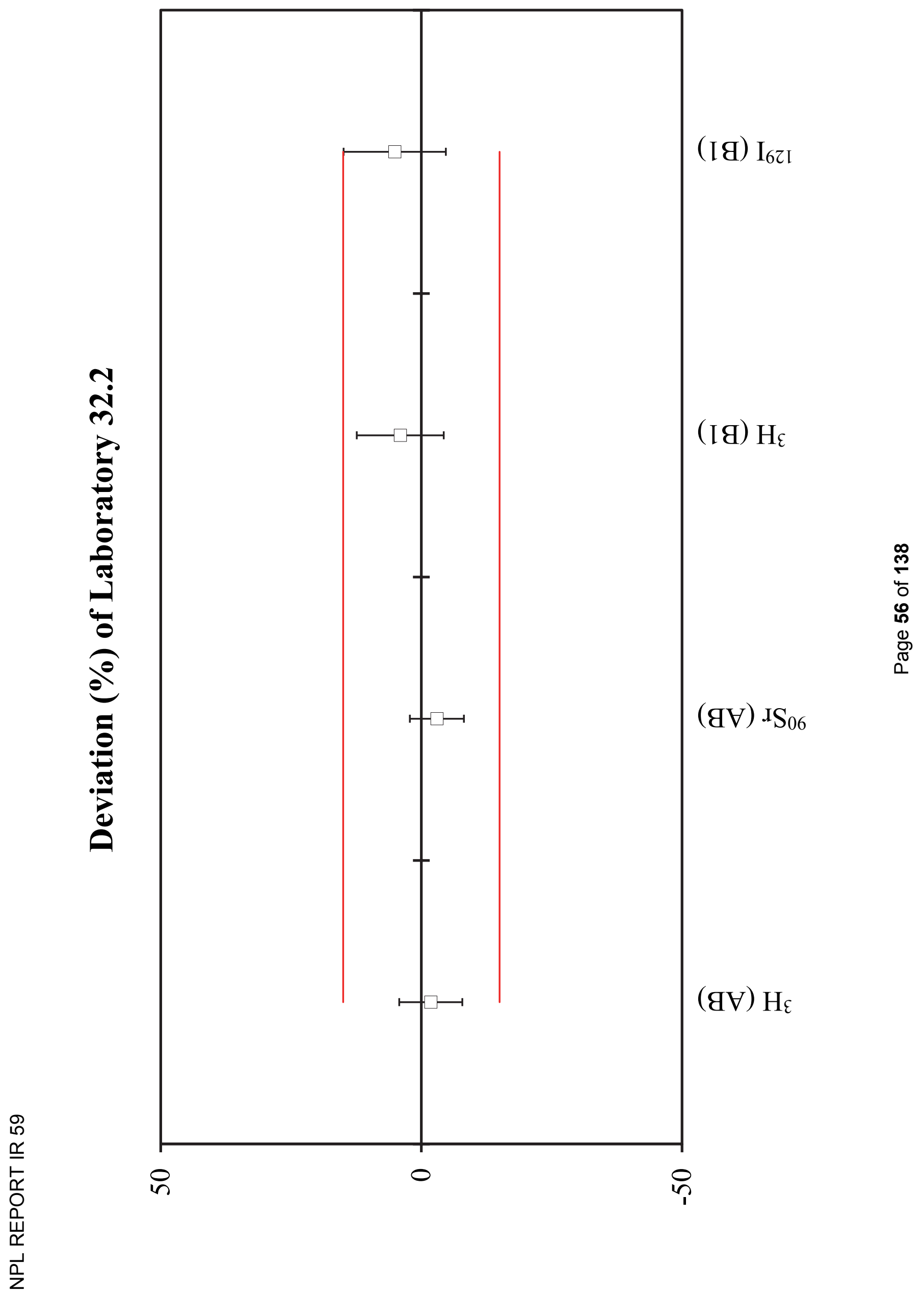




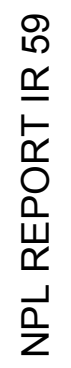

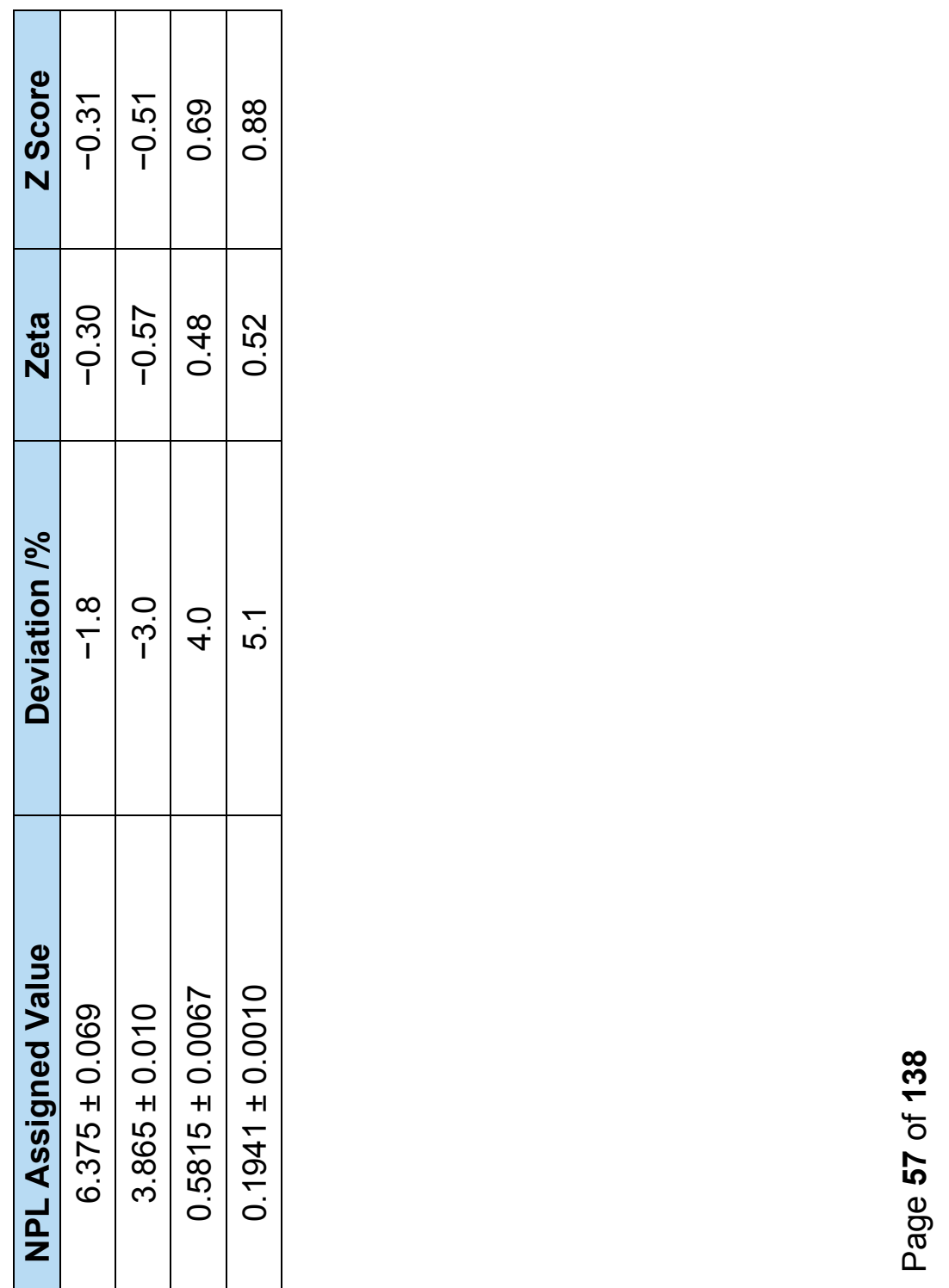

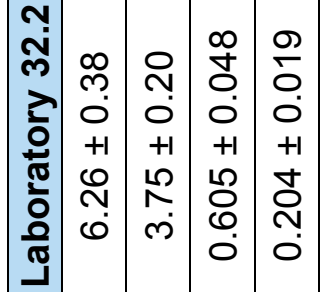

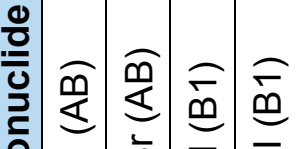

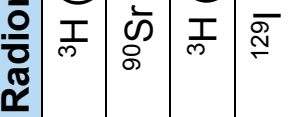




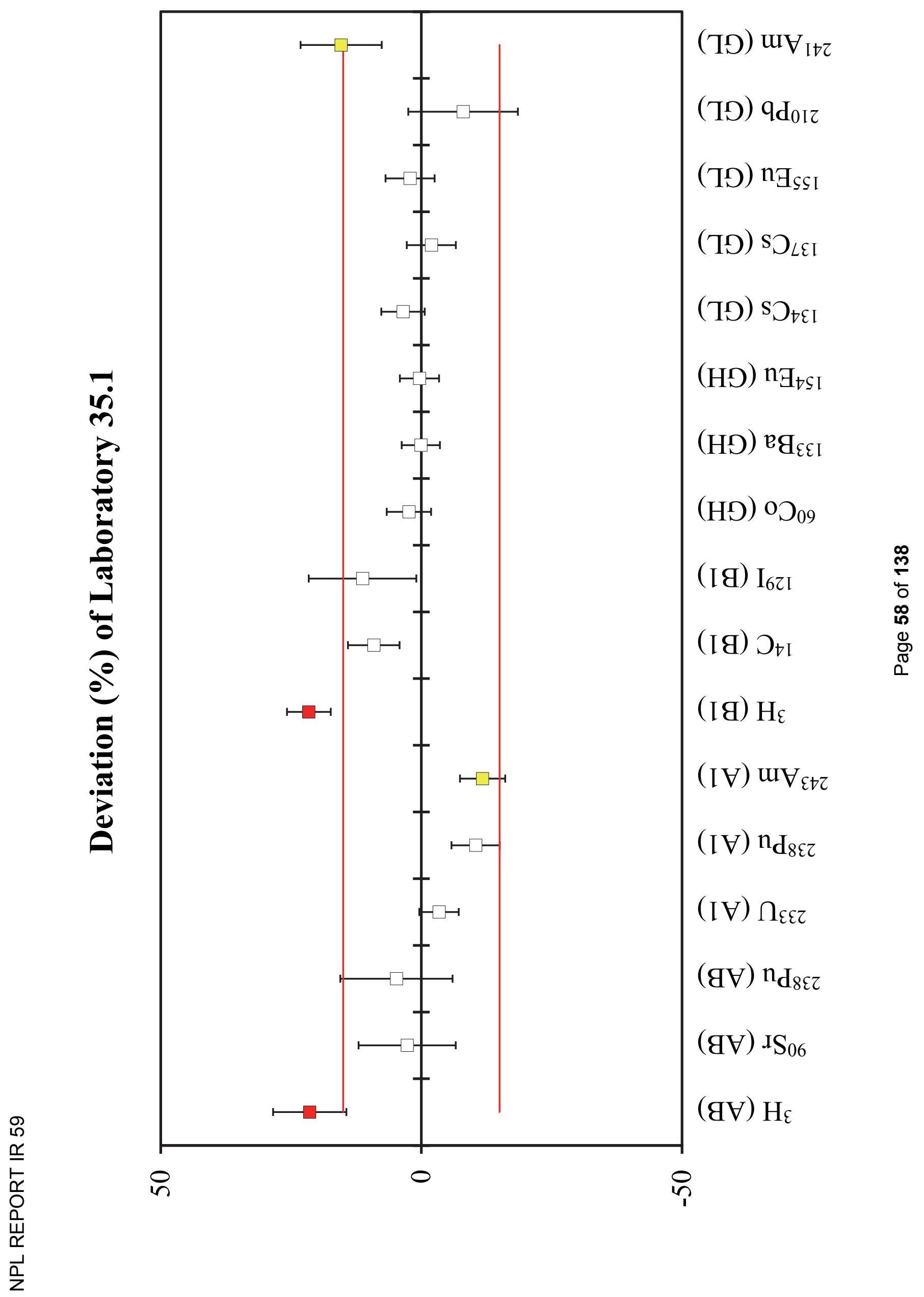




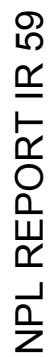

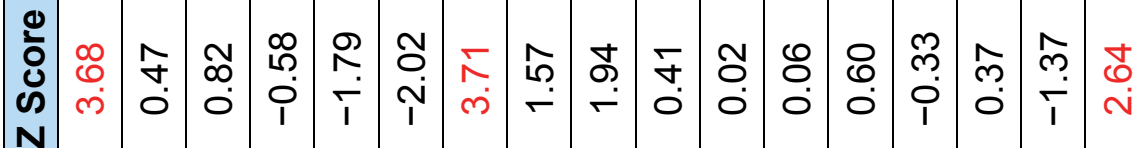

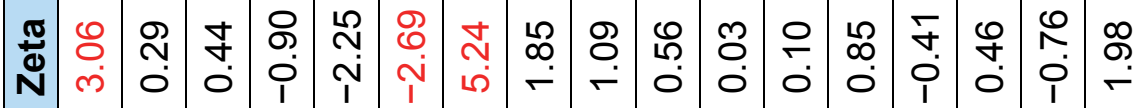

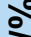

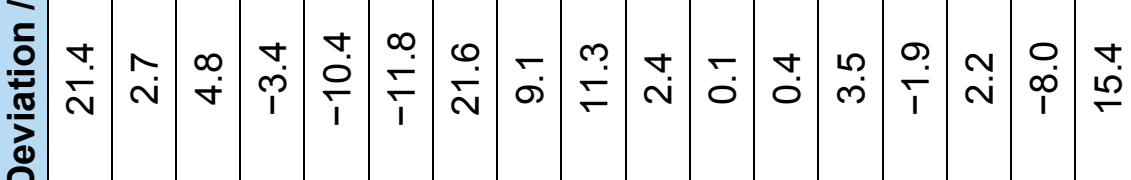

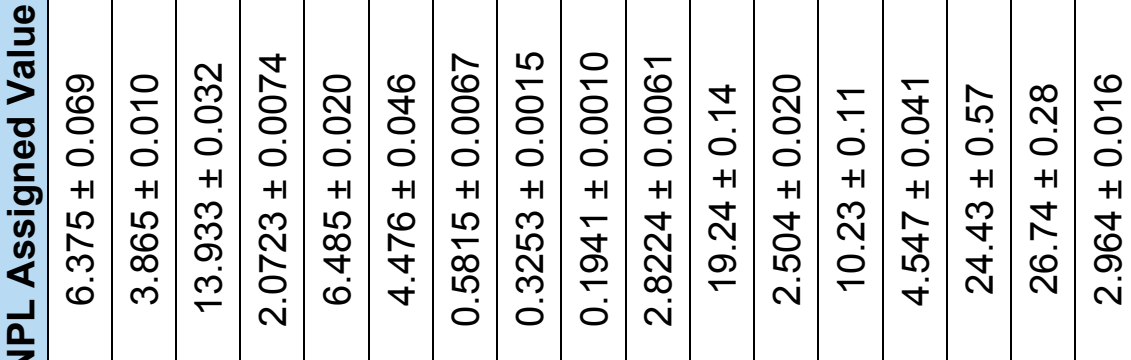

$\stackrel{9}{9}$
$\stackrel{4}{0}$
0
0
0
0
0
0

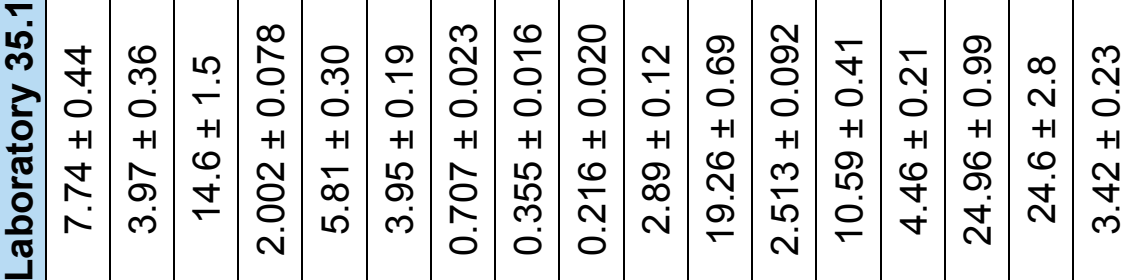

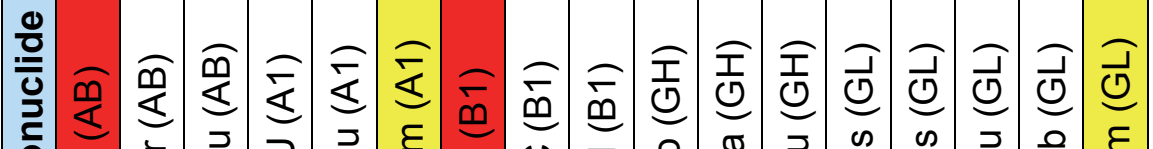

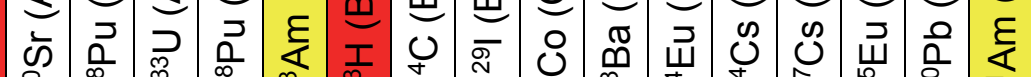
S 


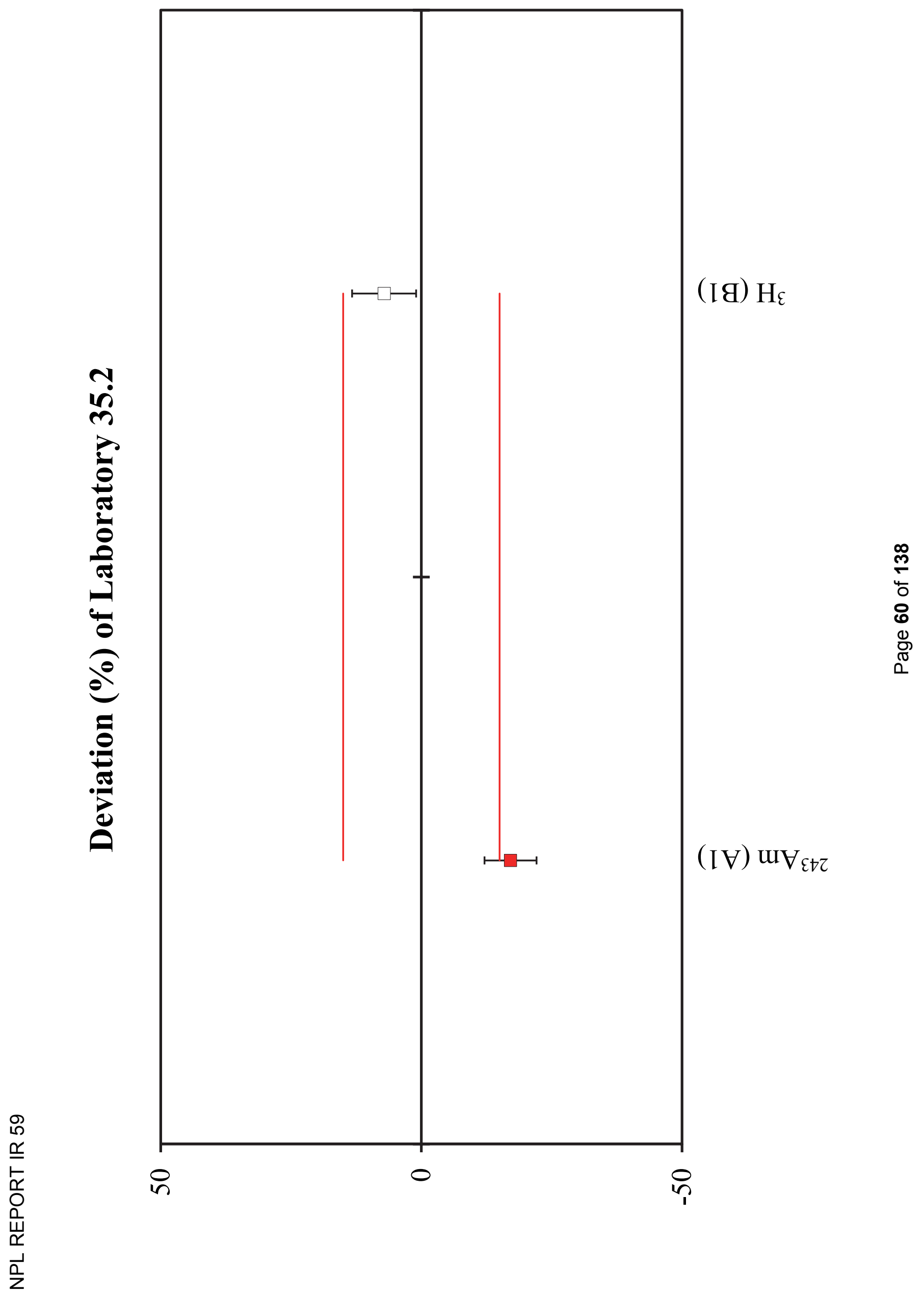




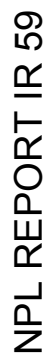

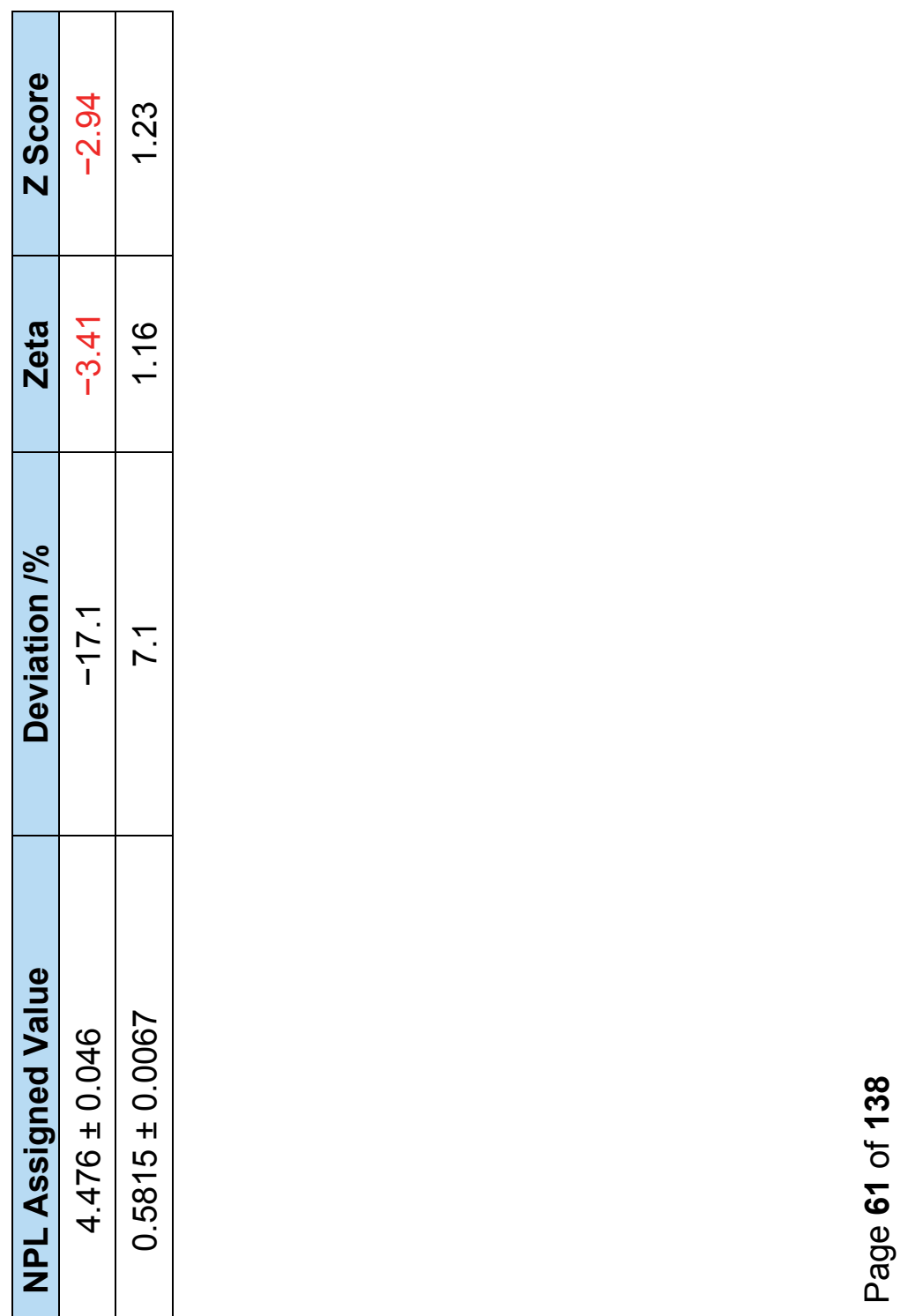

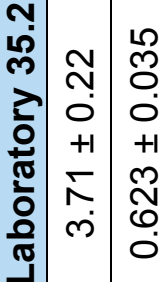

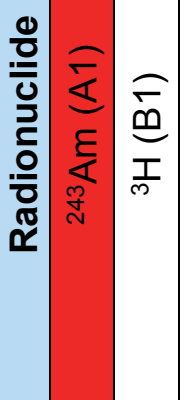




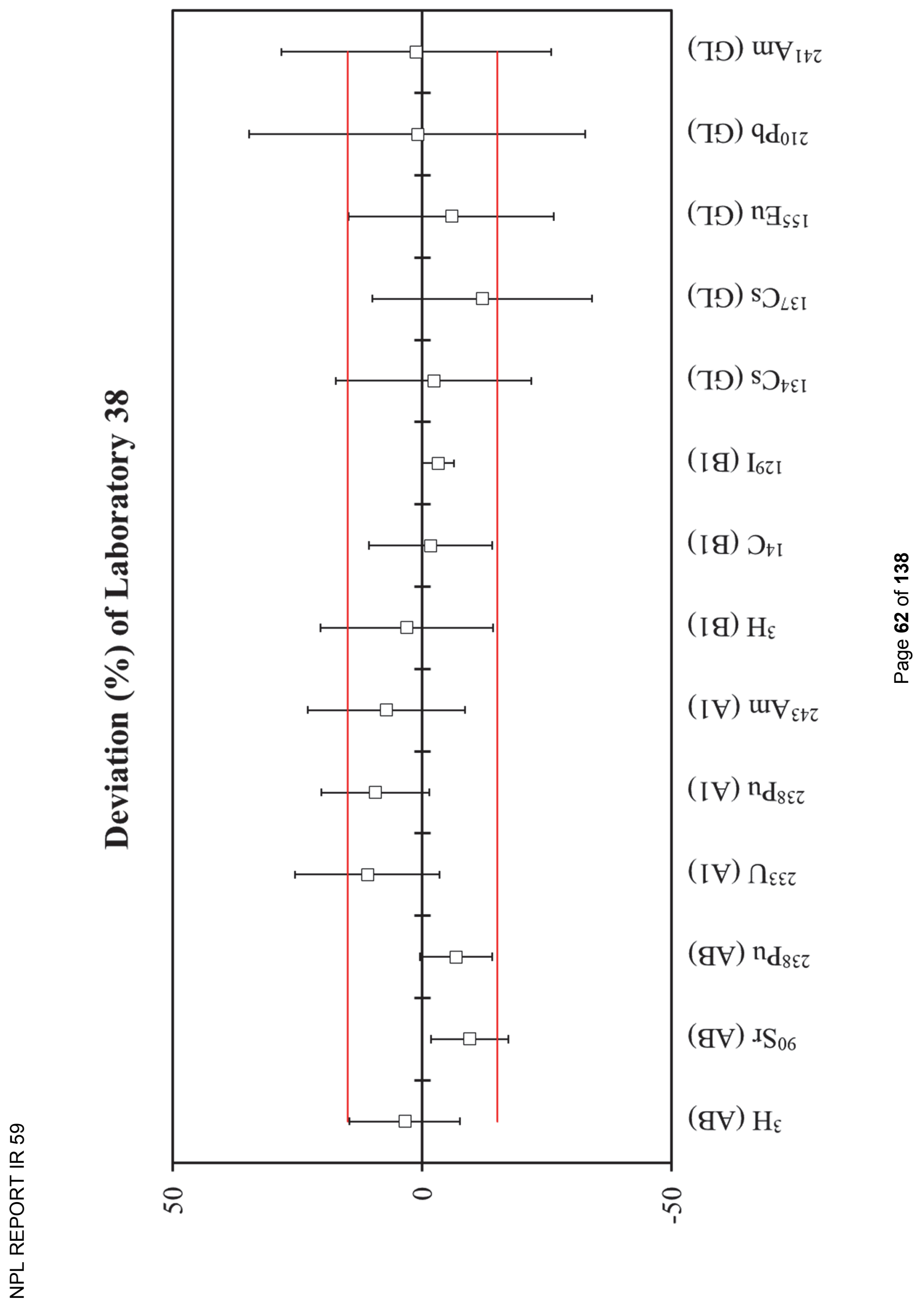




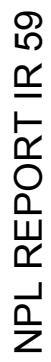

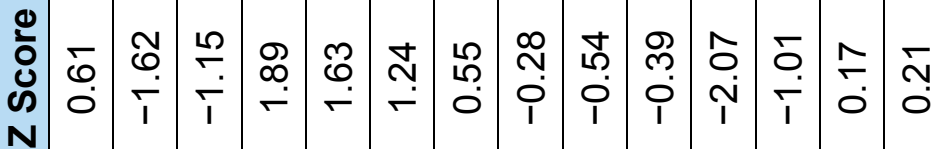

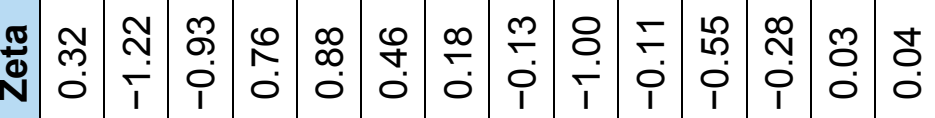

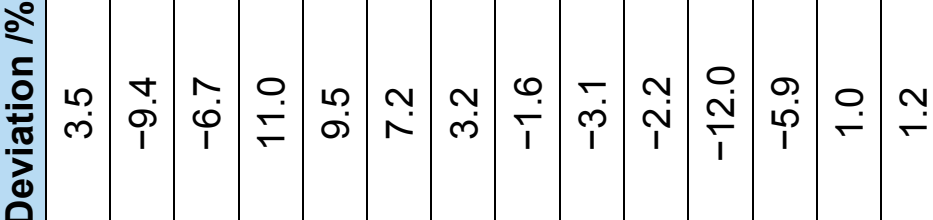

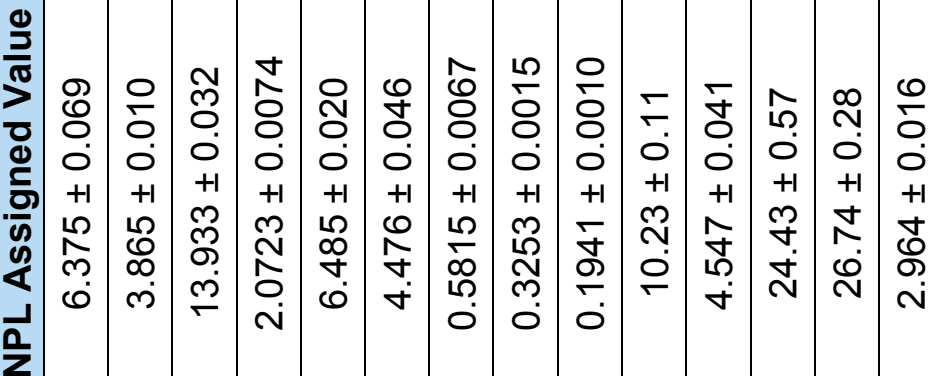

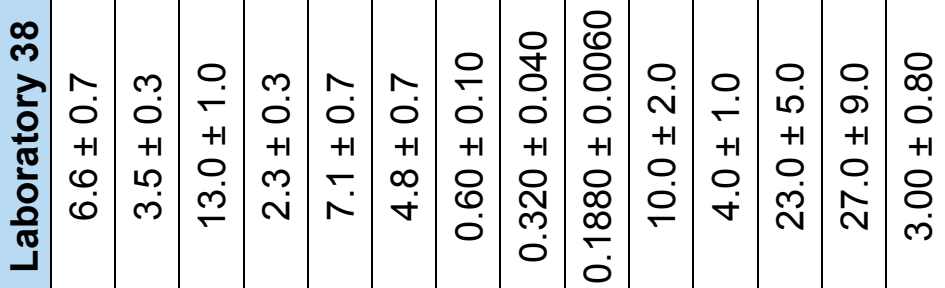

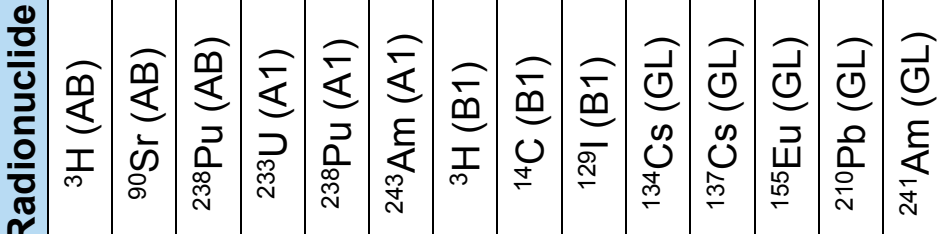




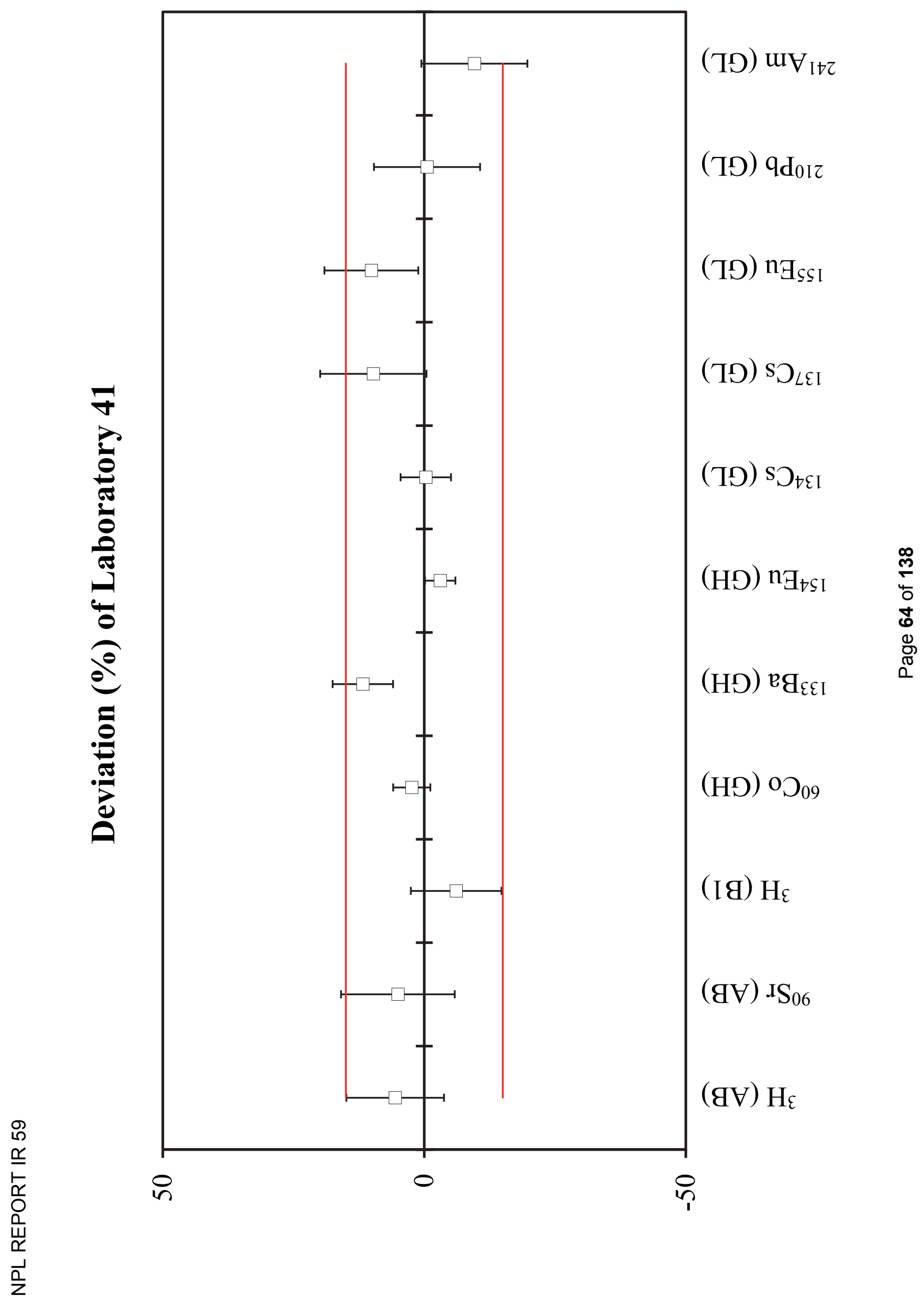




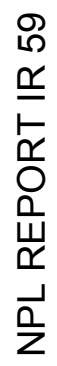

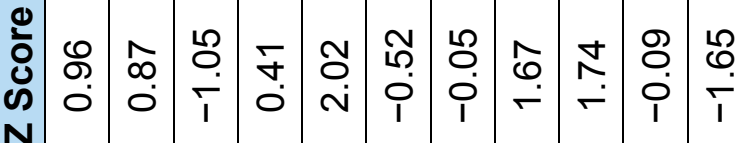

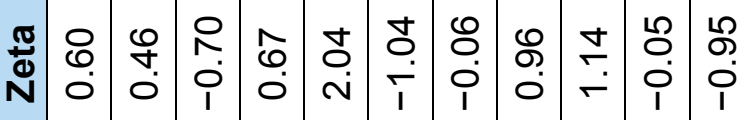

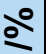

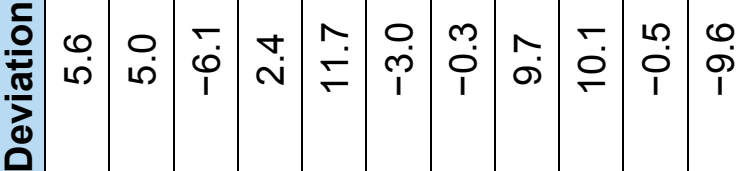

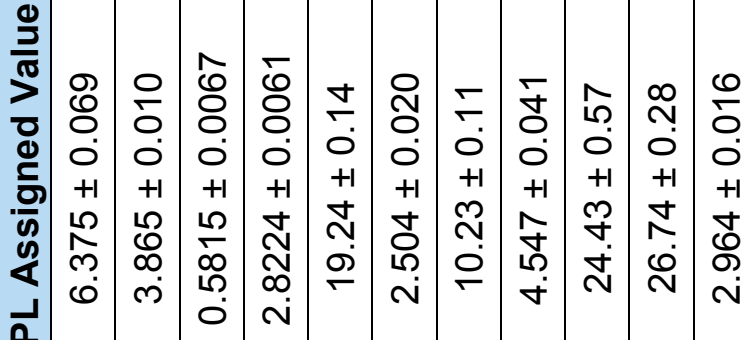

$\infty$
$\stackrel{2}{7}$
$\overleftarrow{0}$
10
0
0
0
0
0

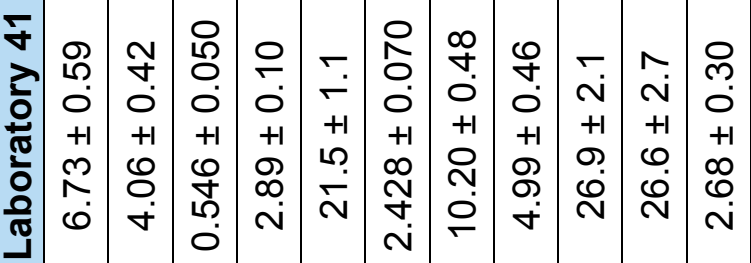

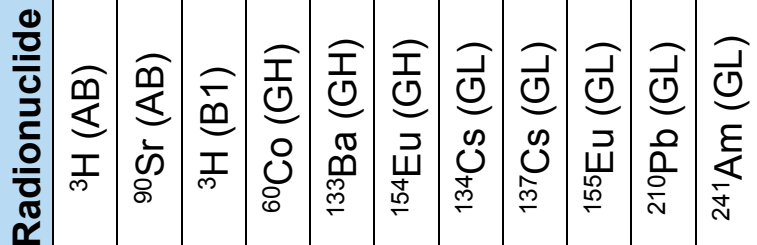




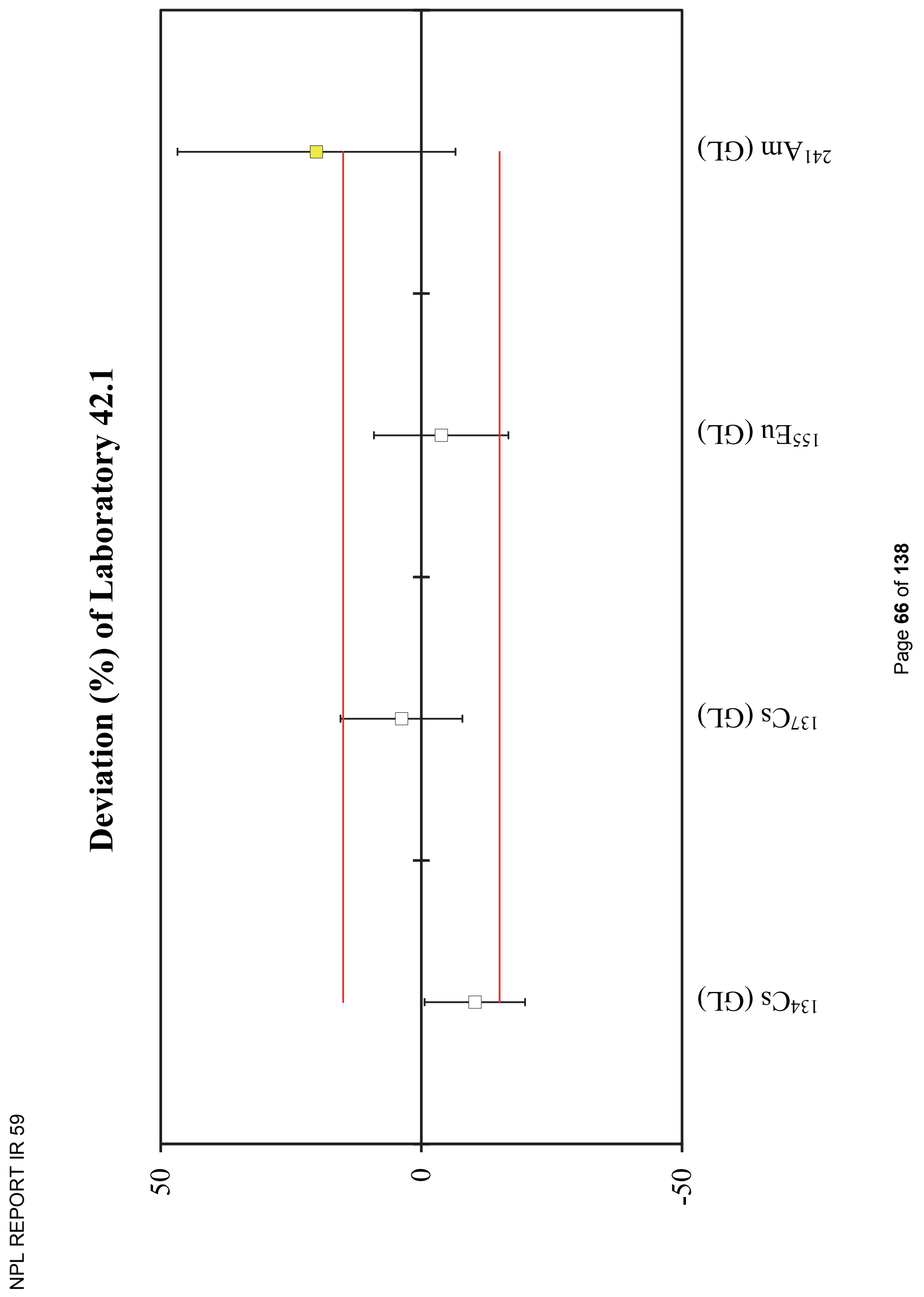




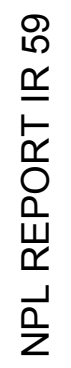

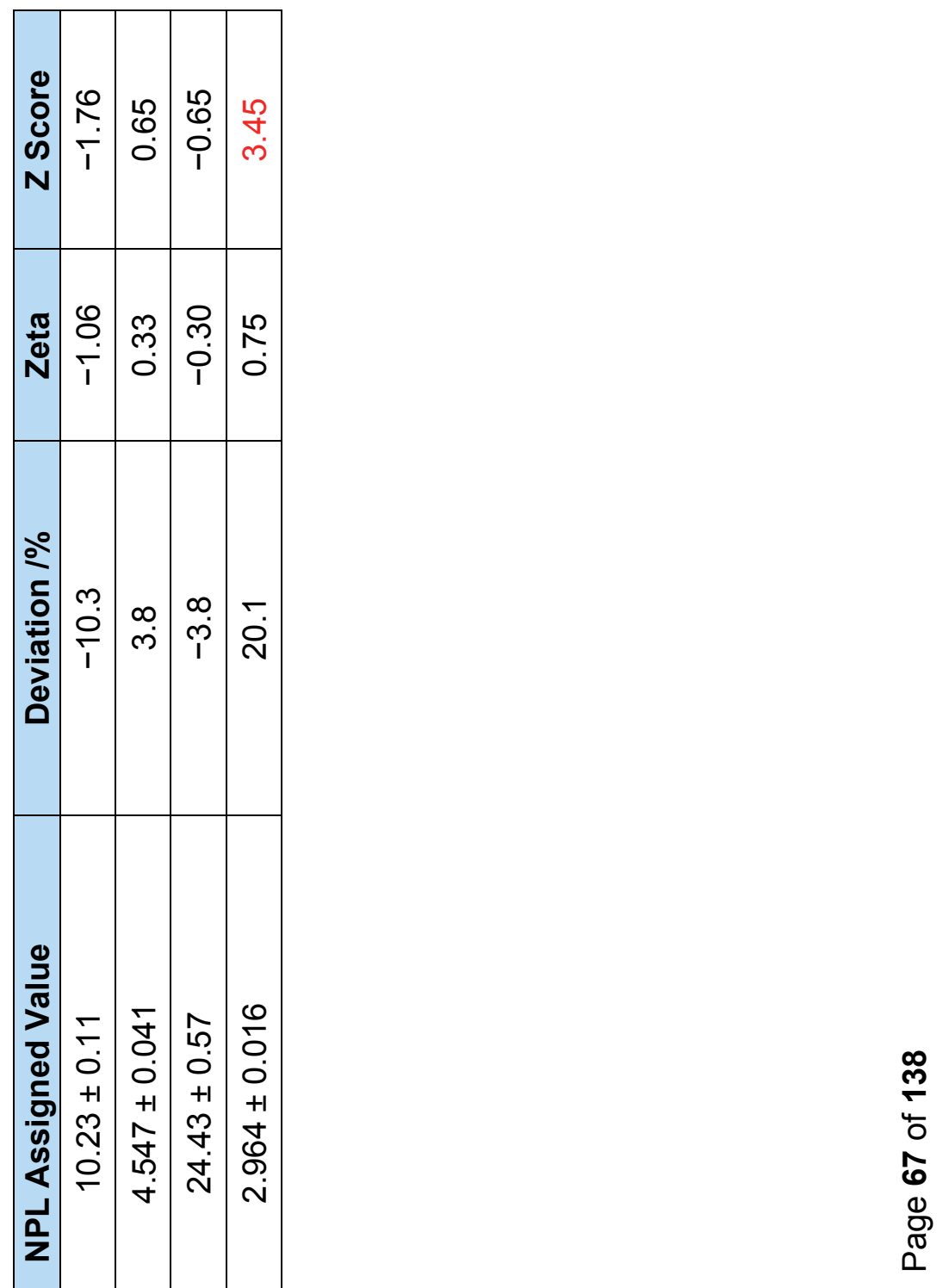

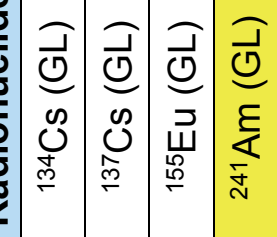




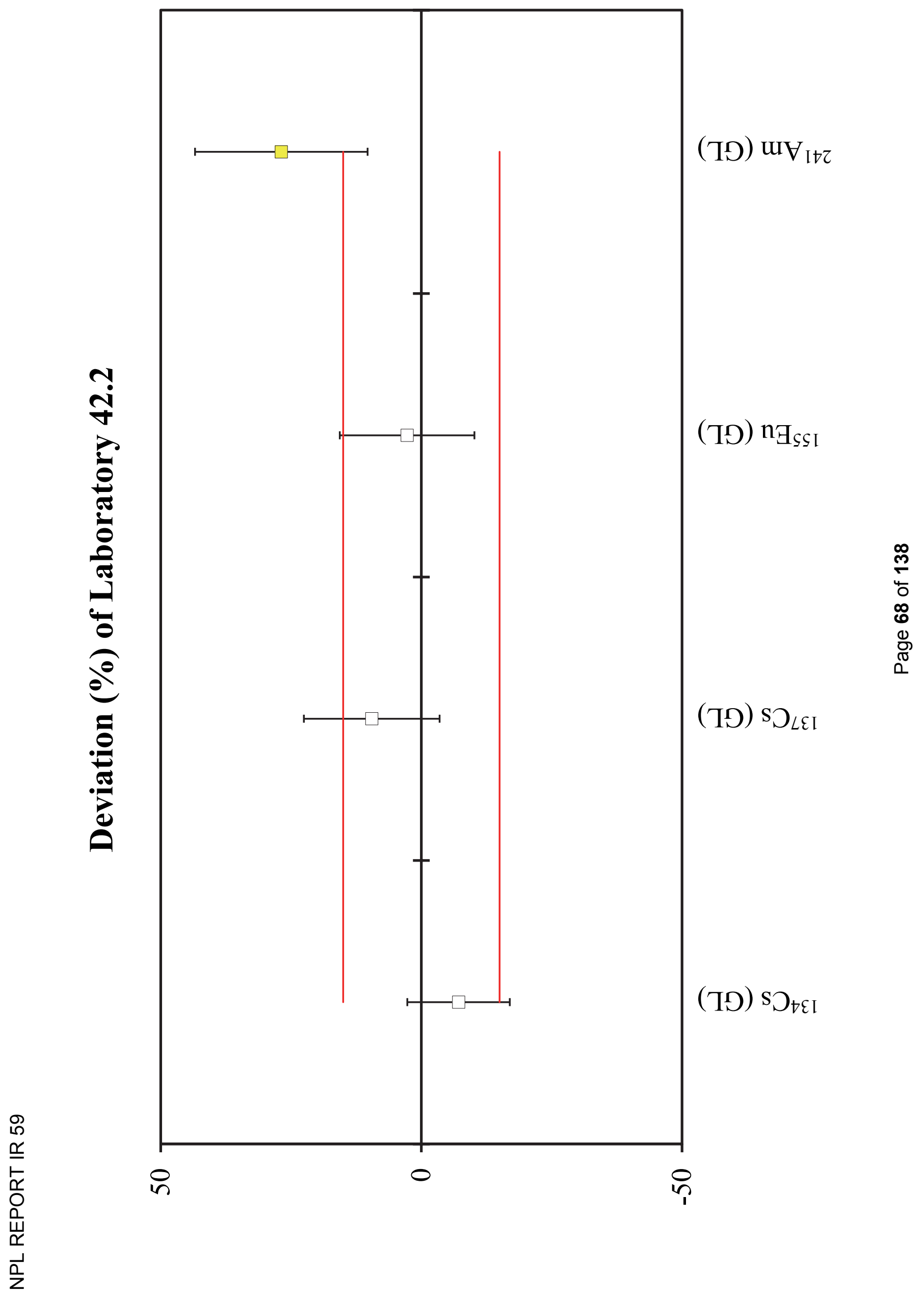




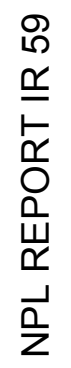

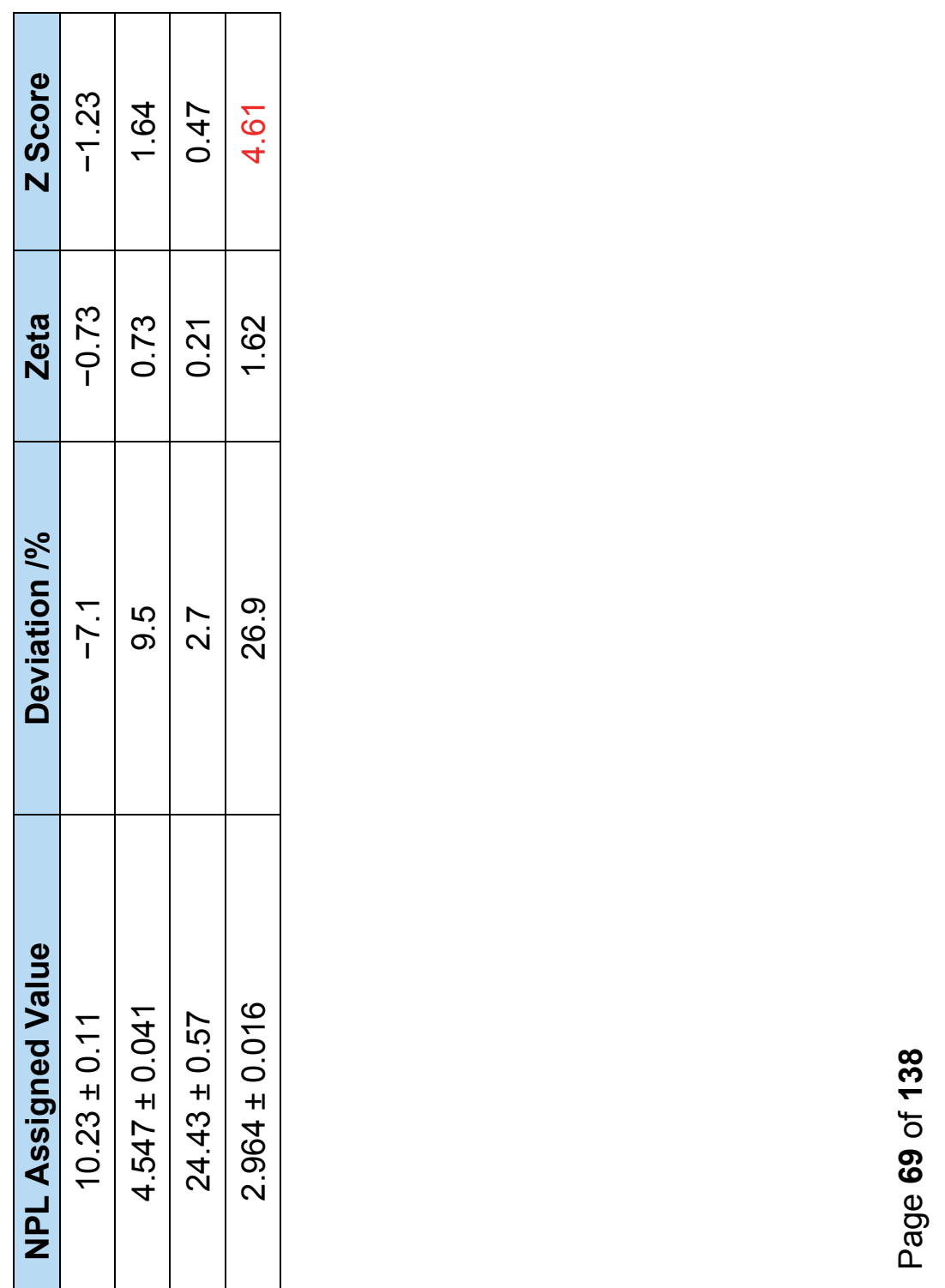




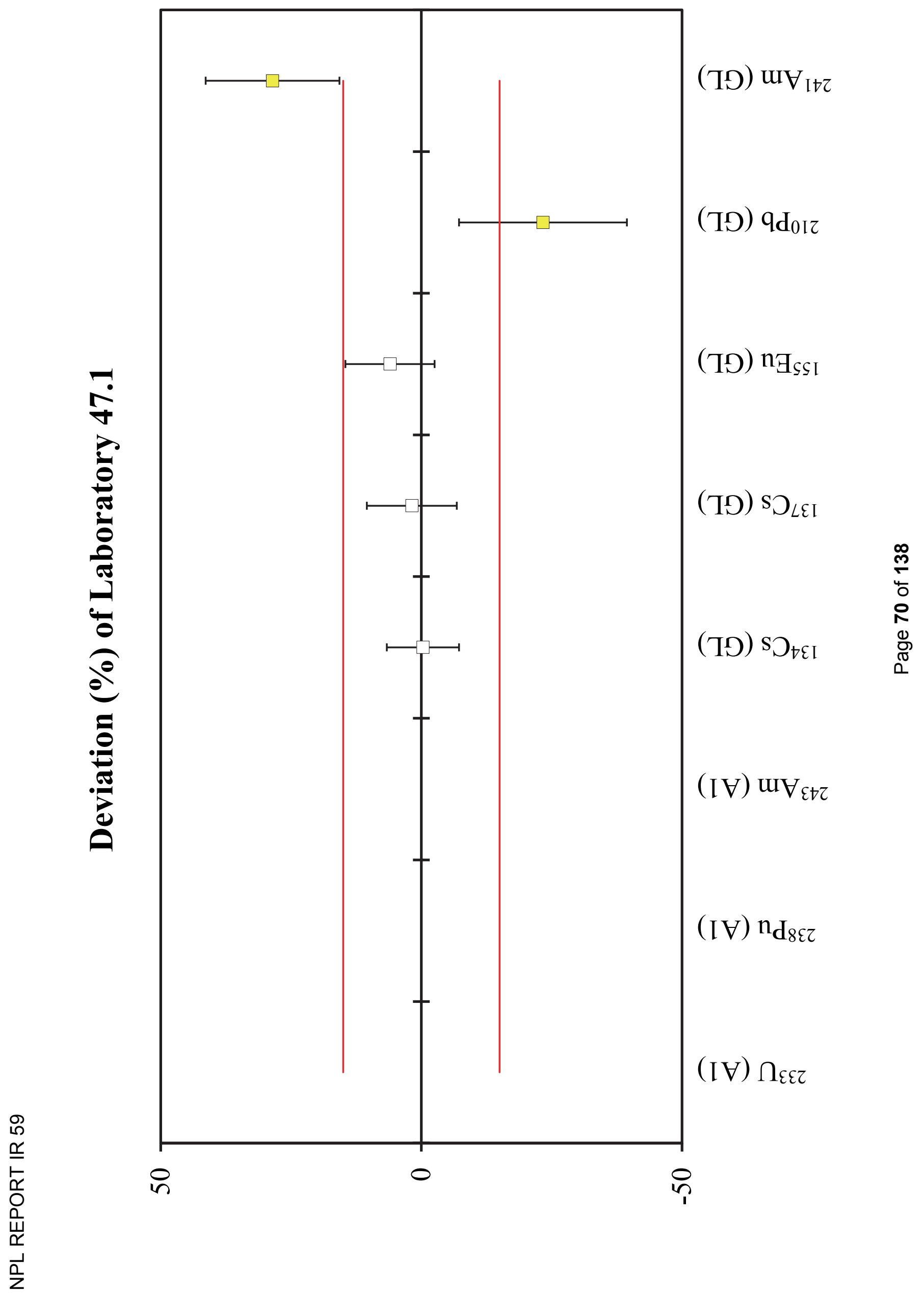




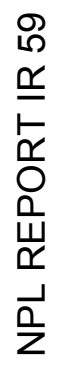

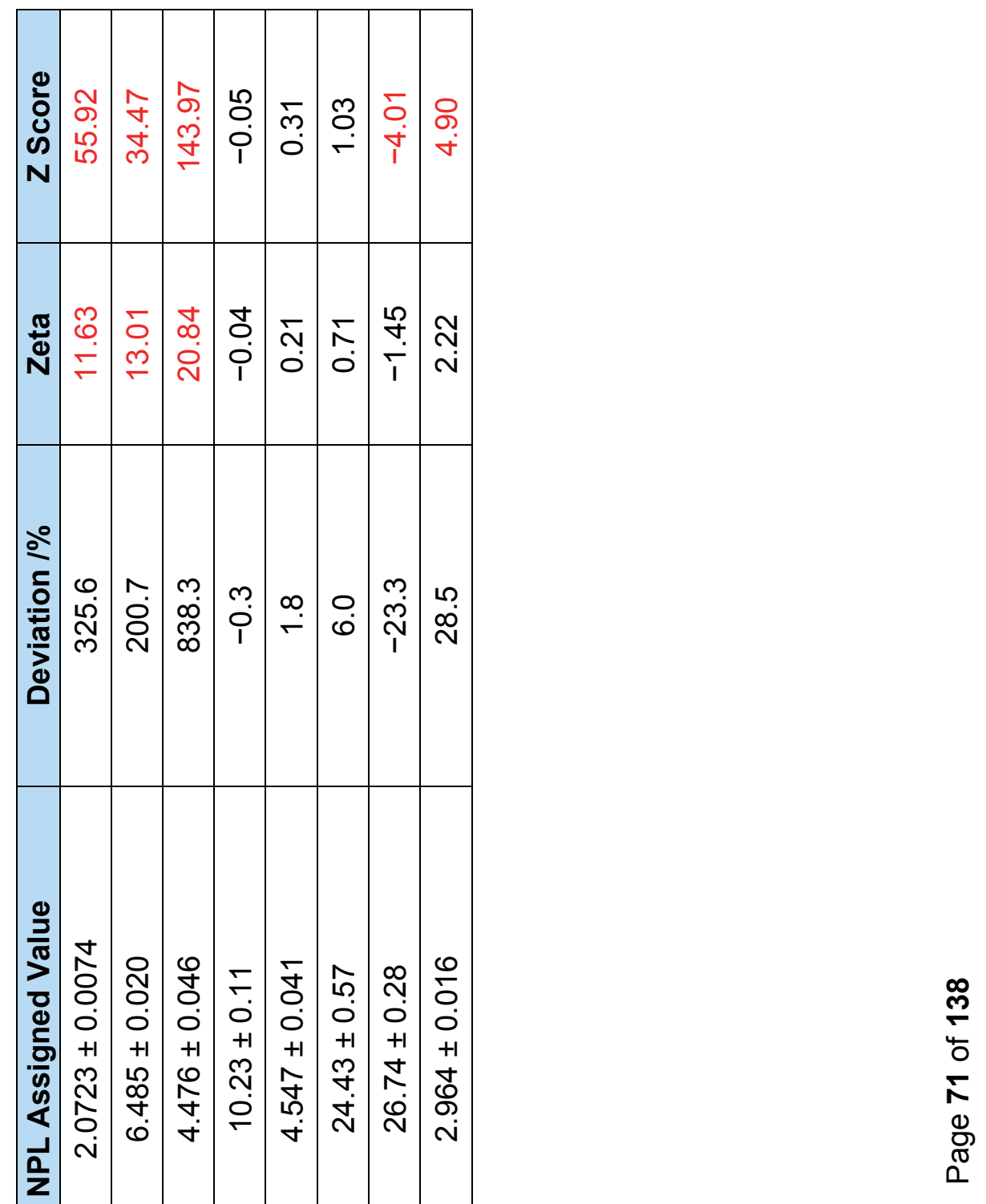

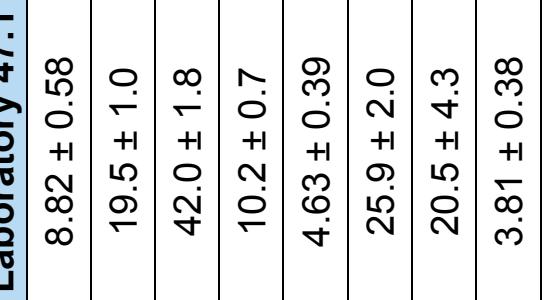

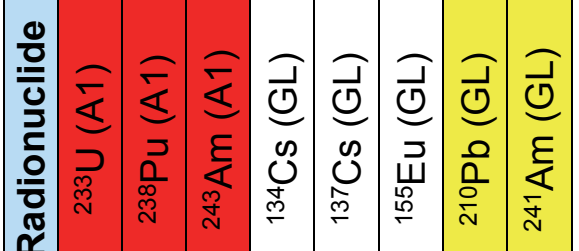




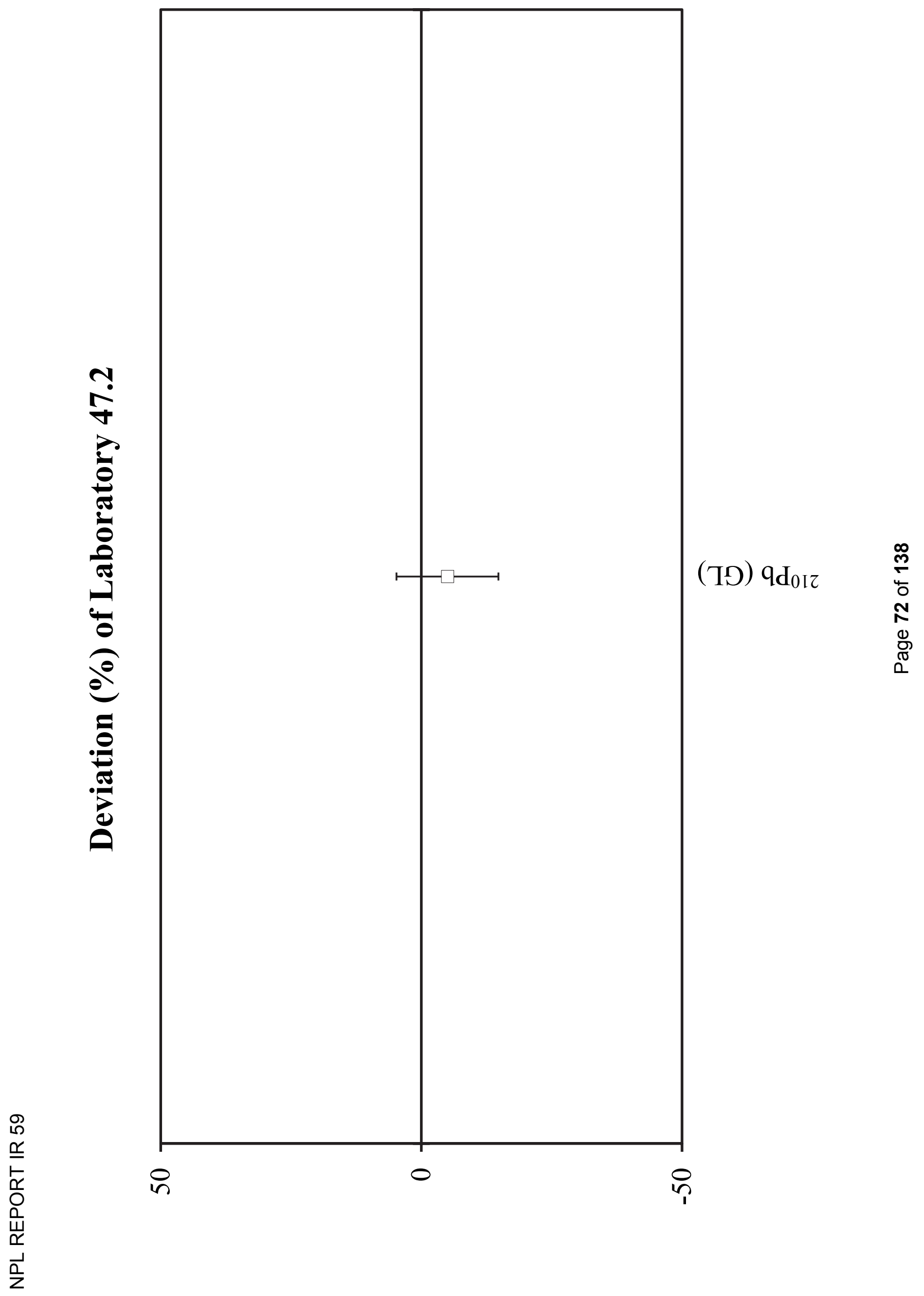




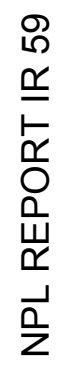
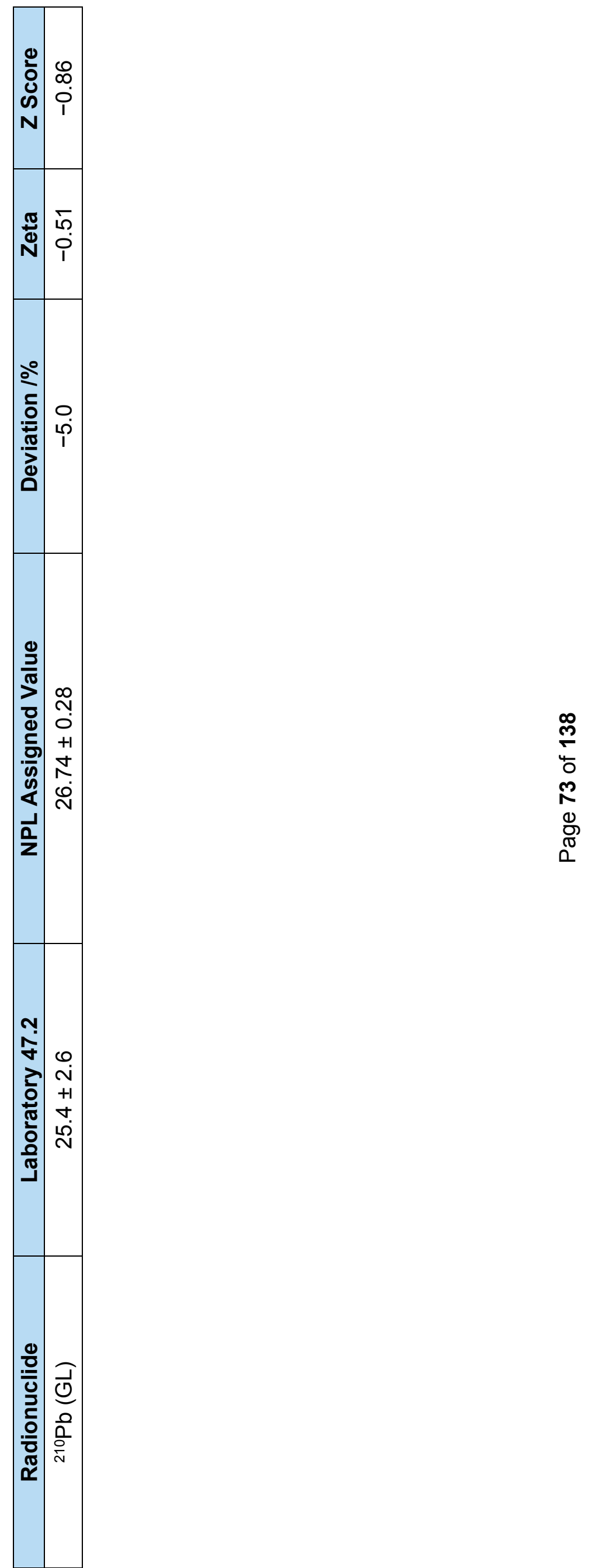


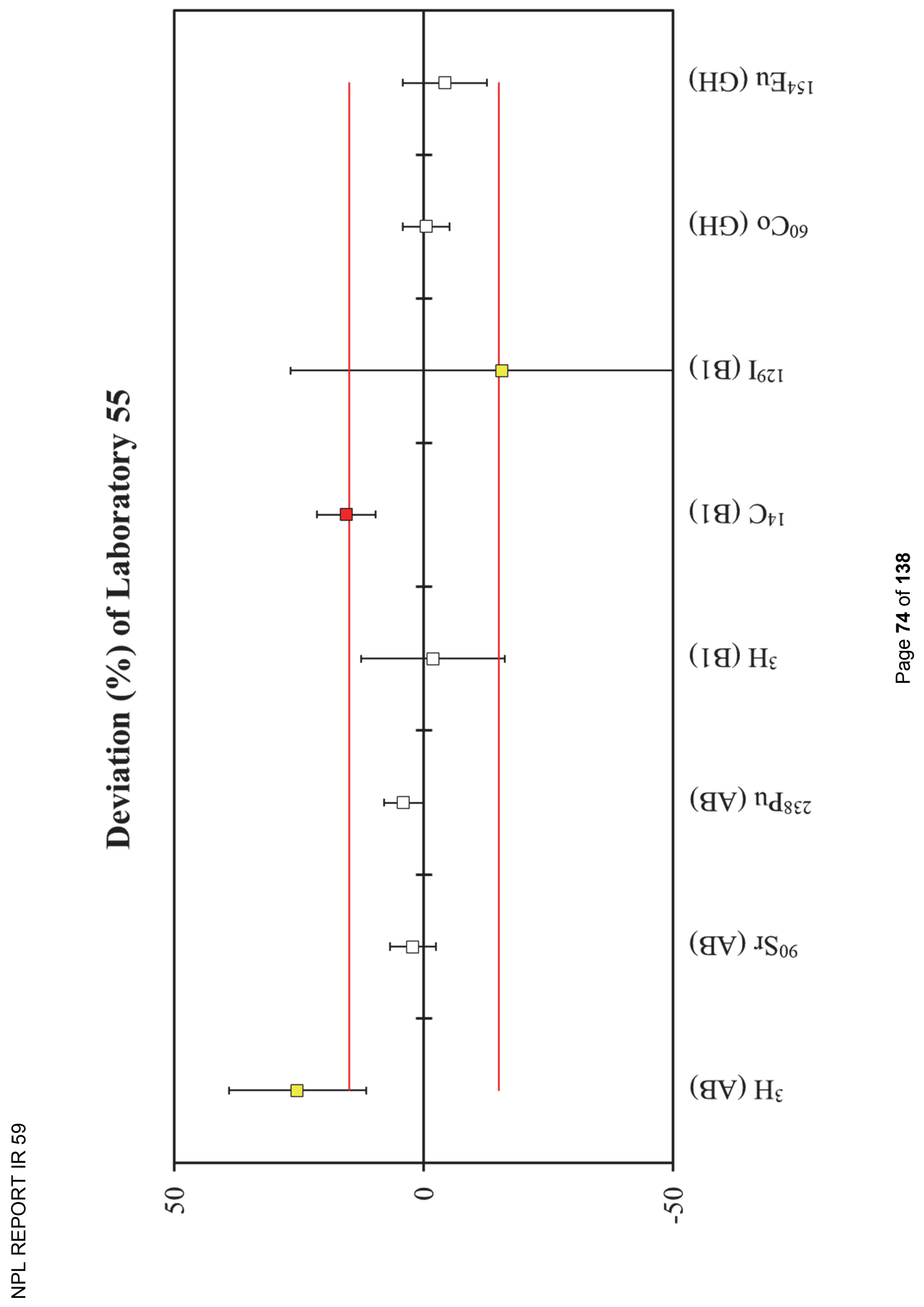




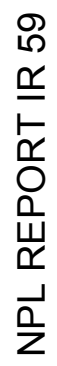

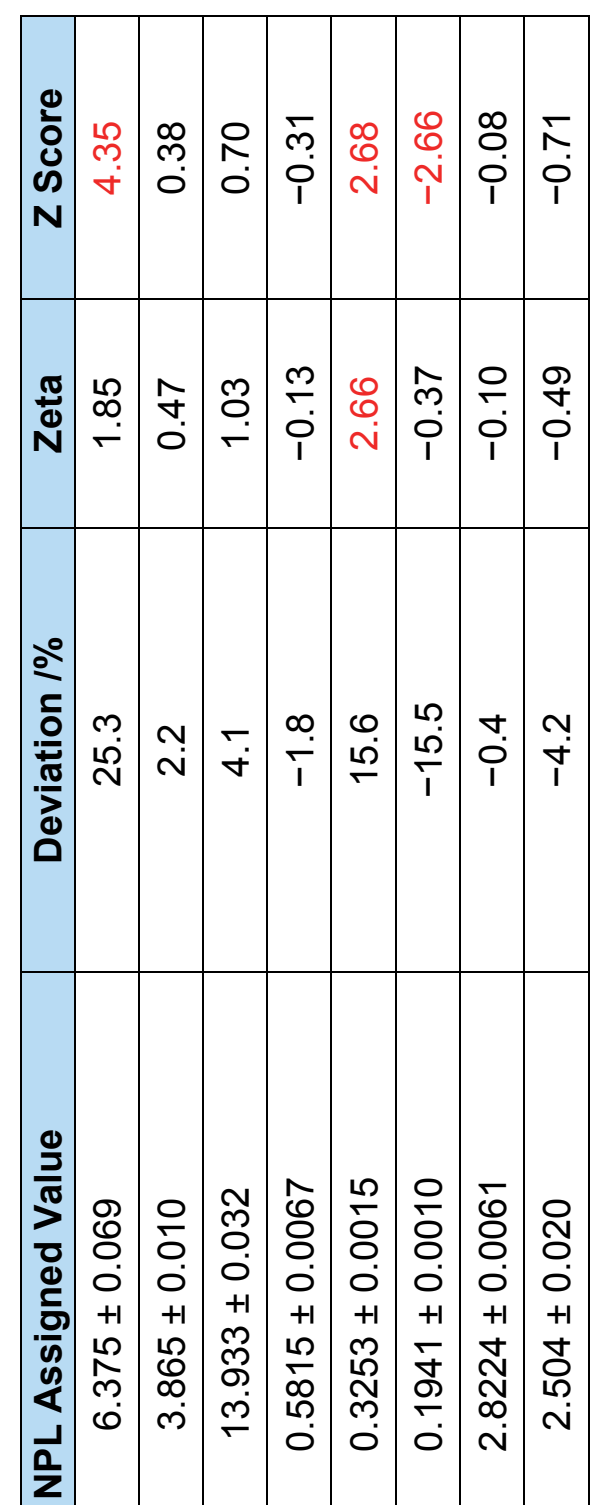

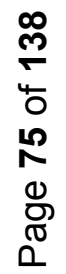

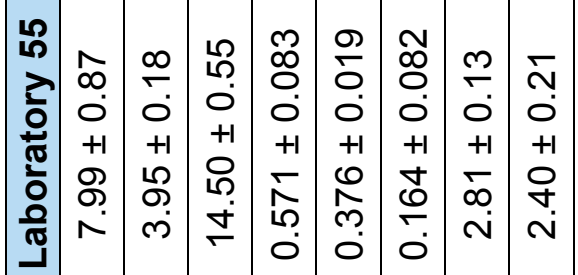

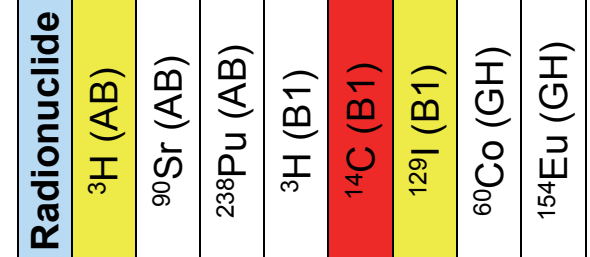




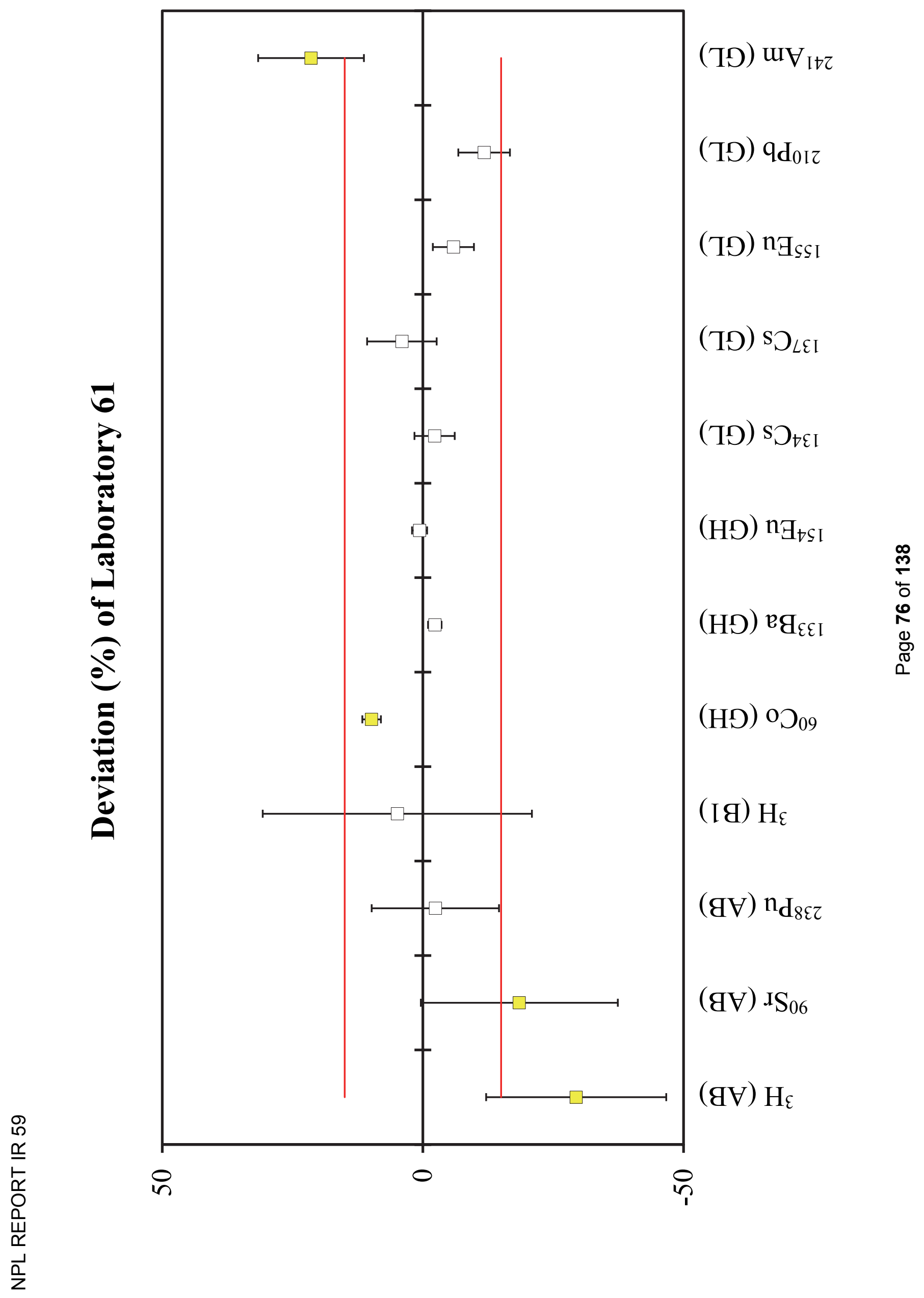




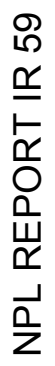

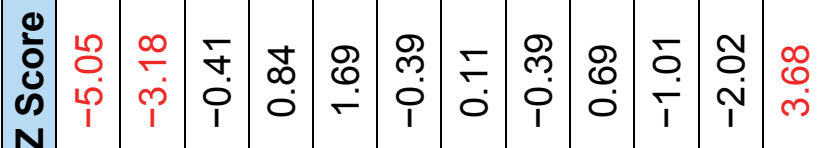

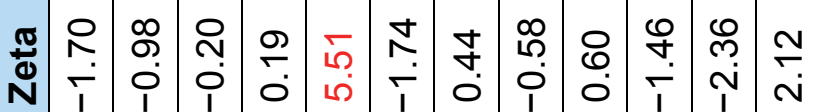

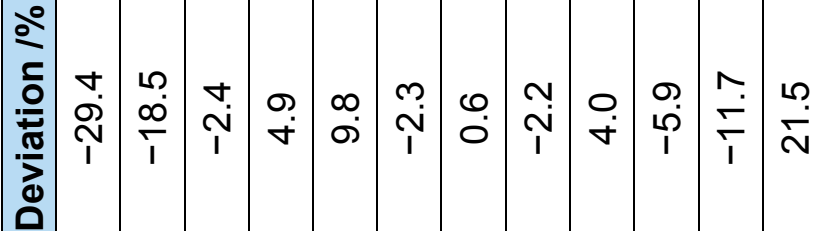

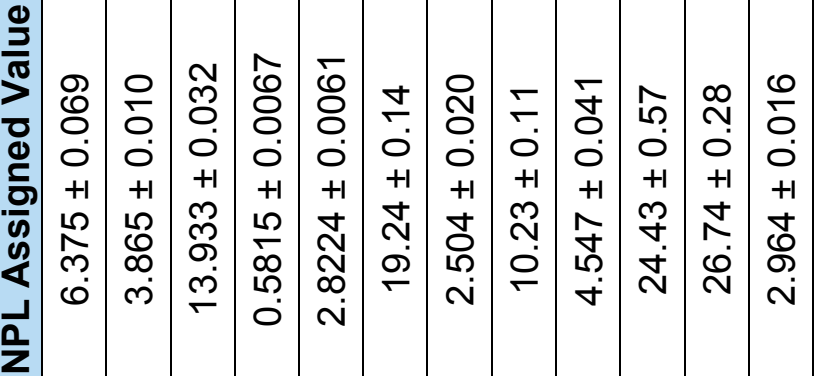

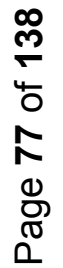

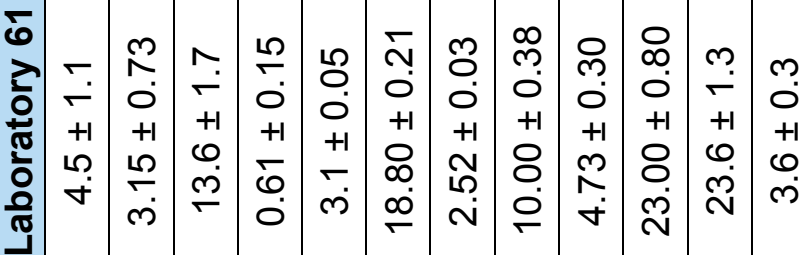

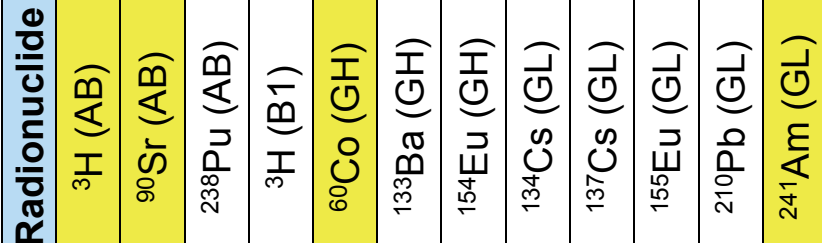




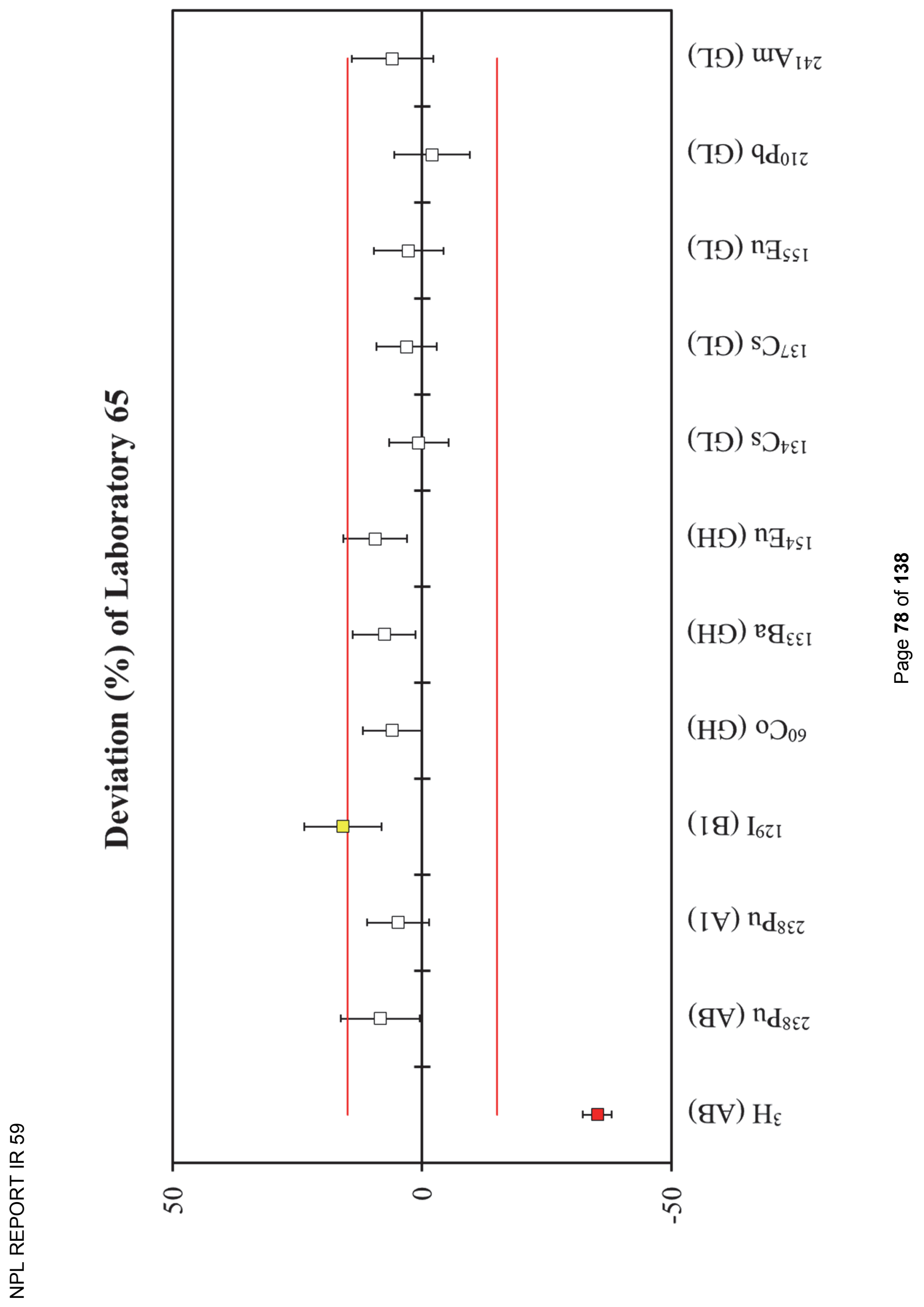




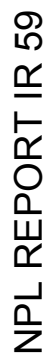

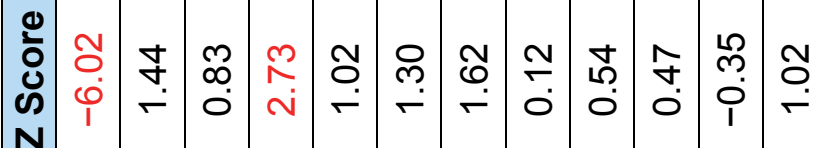

蛋

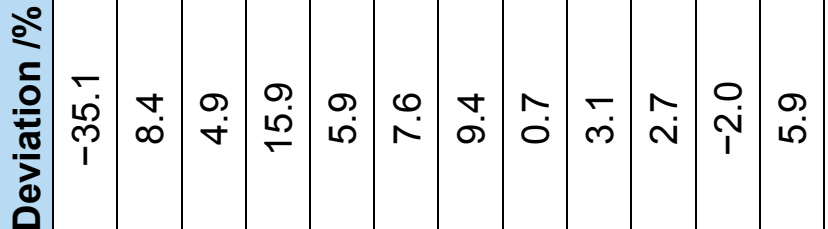

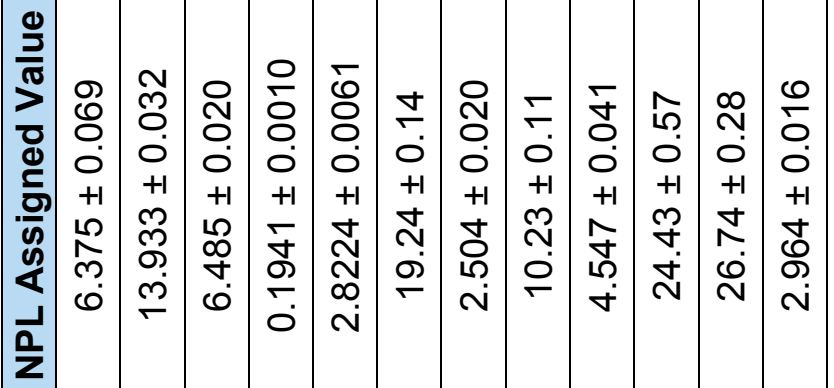

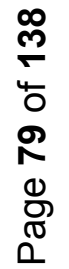

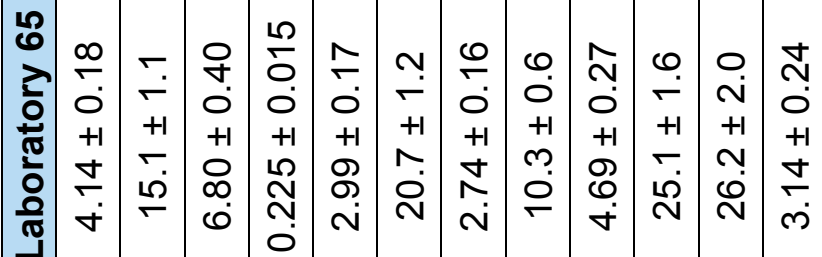

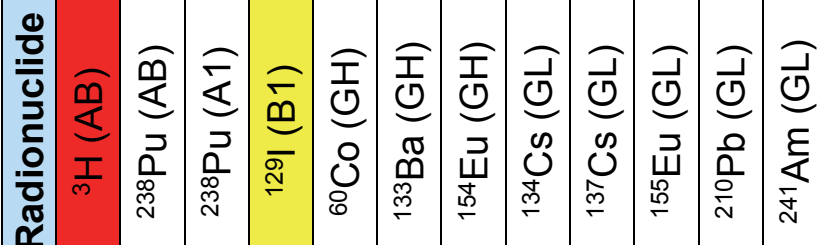




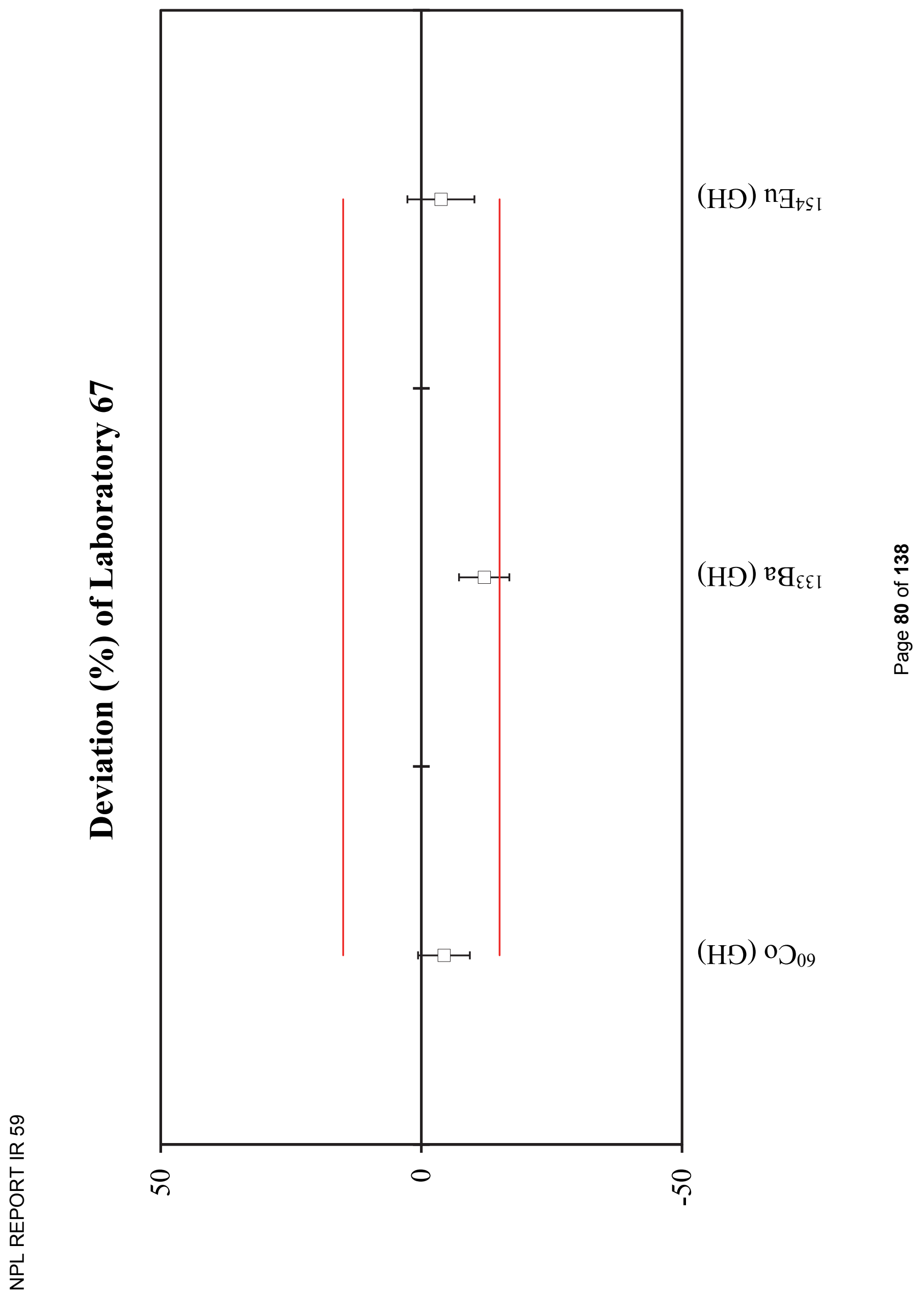




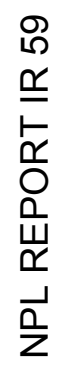

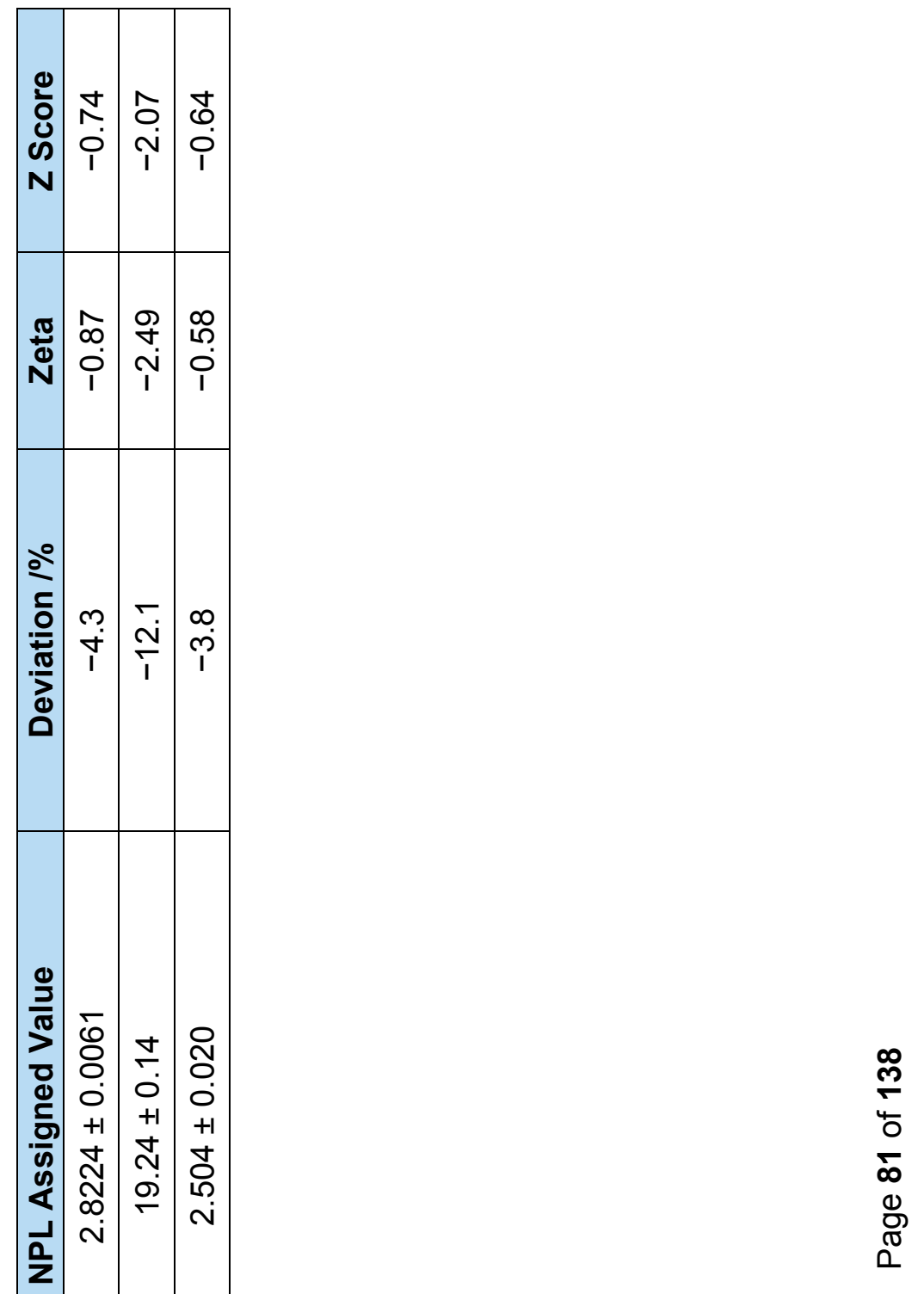

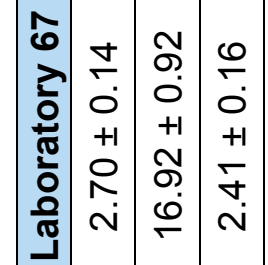

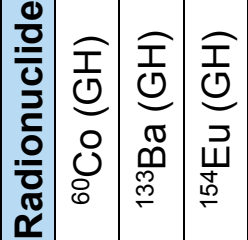




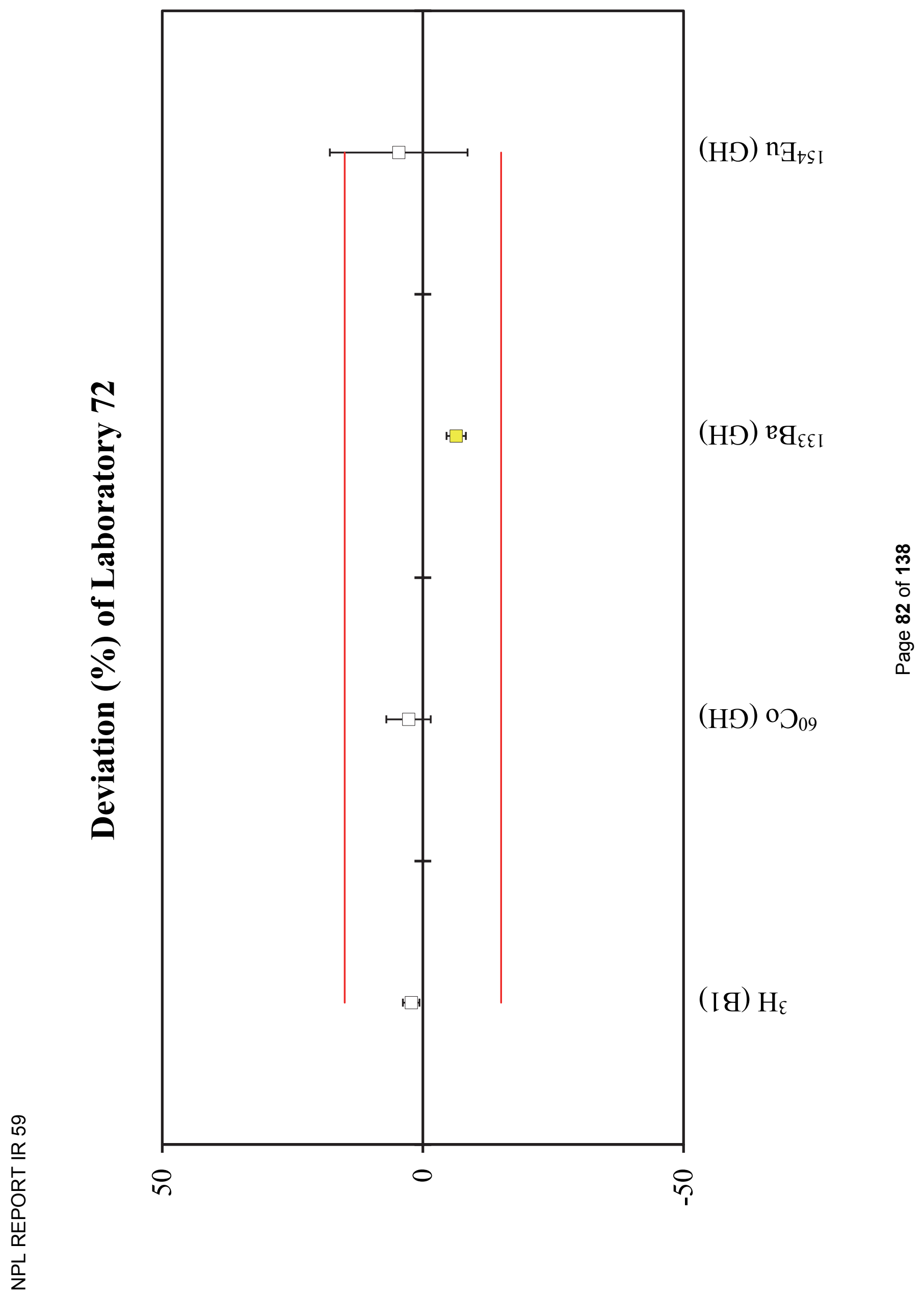




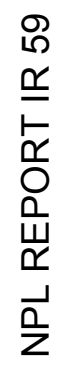

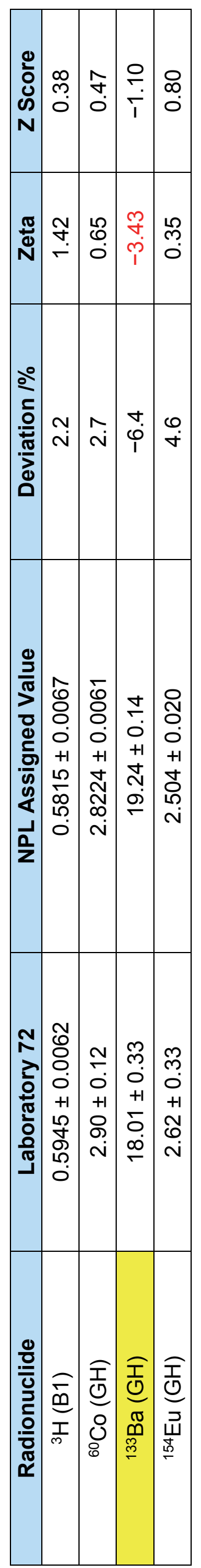

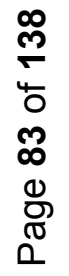




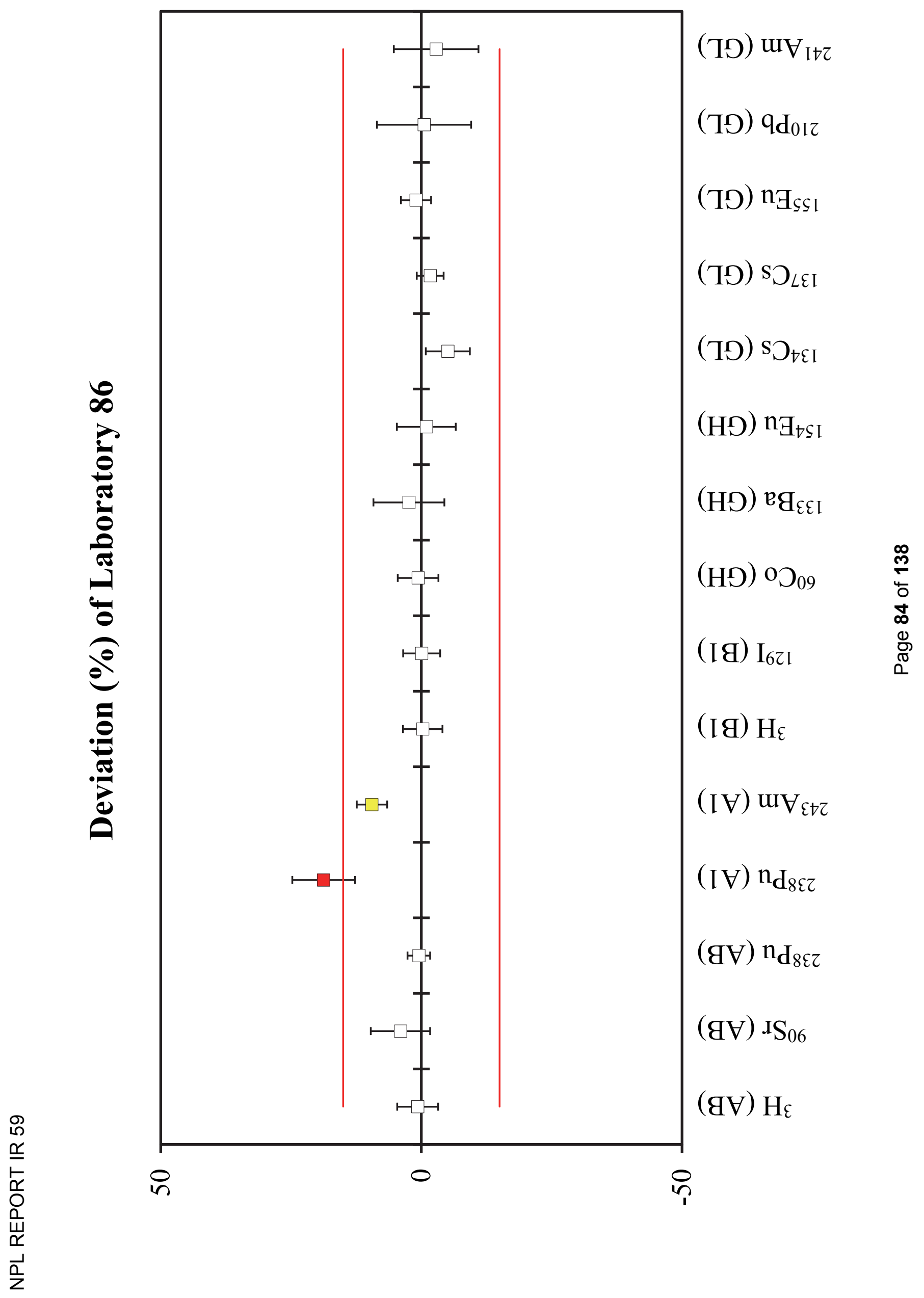




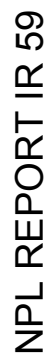

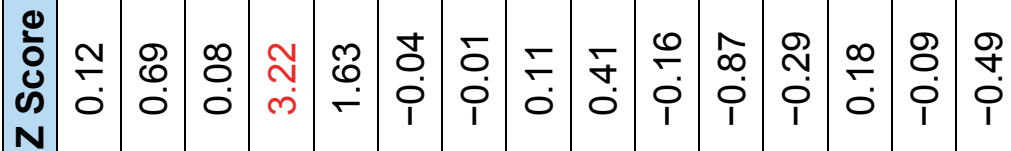

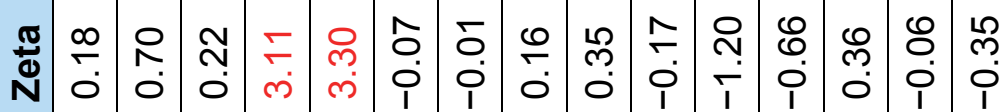

๑

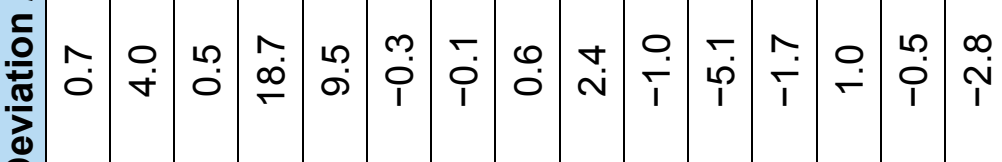

告

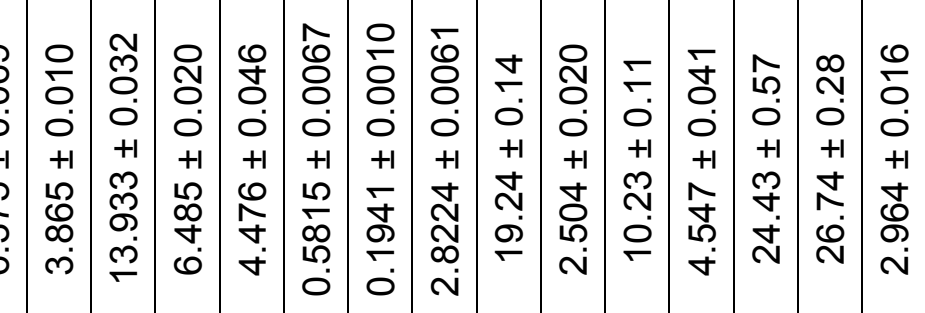

(2)

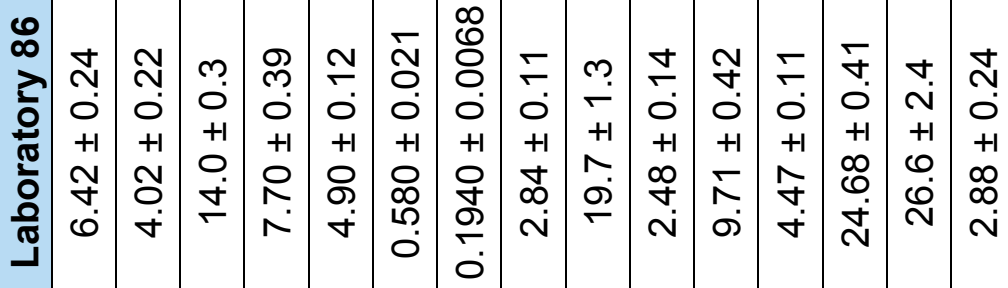

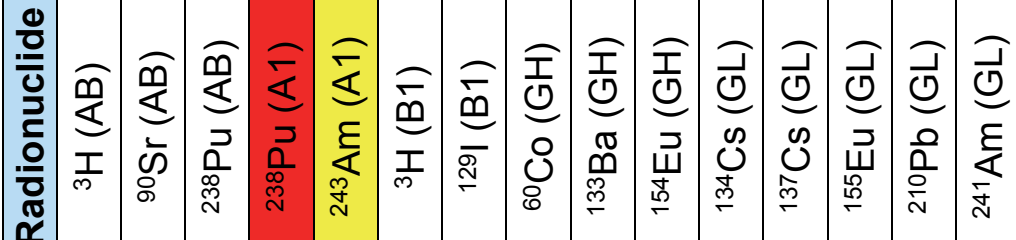

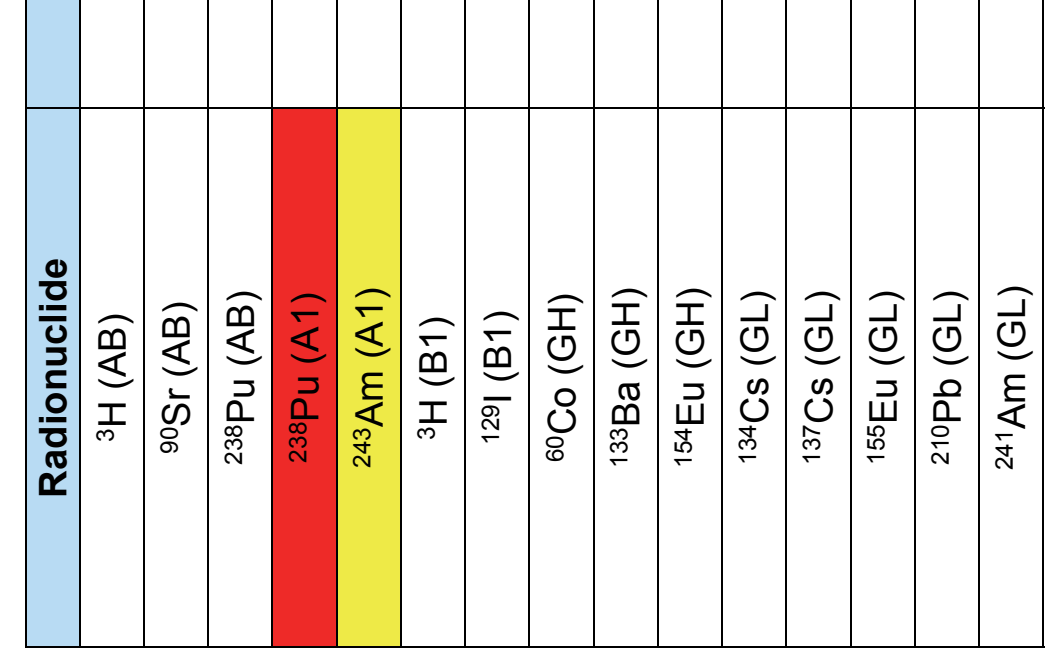




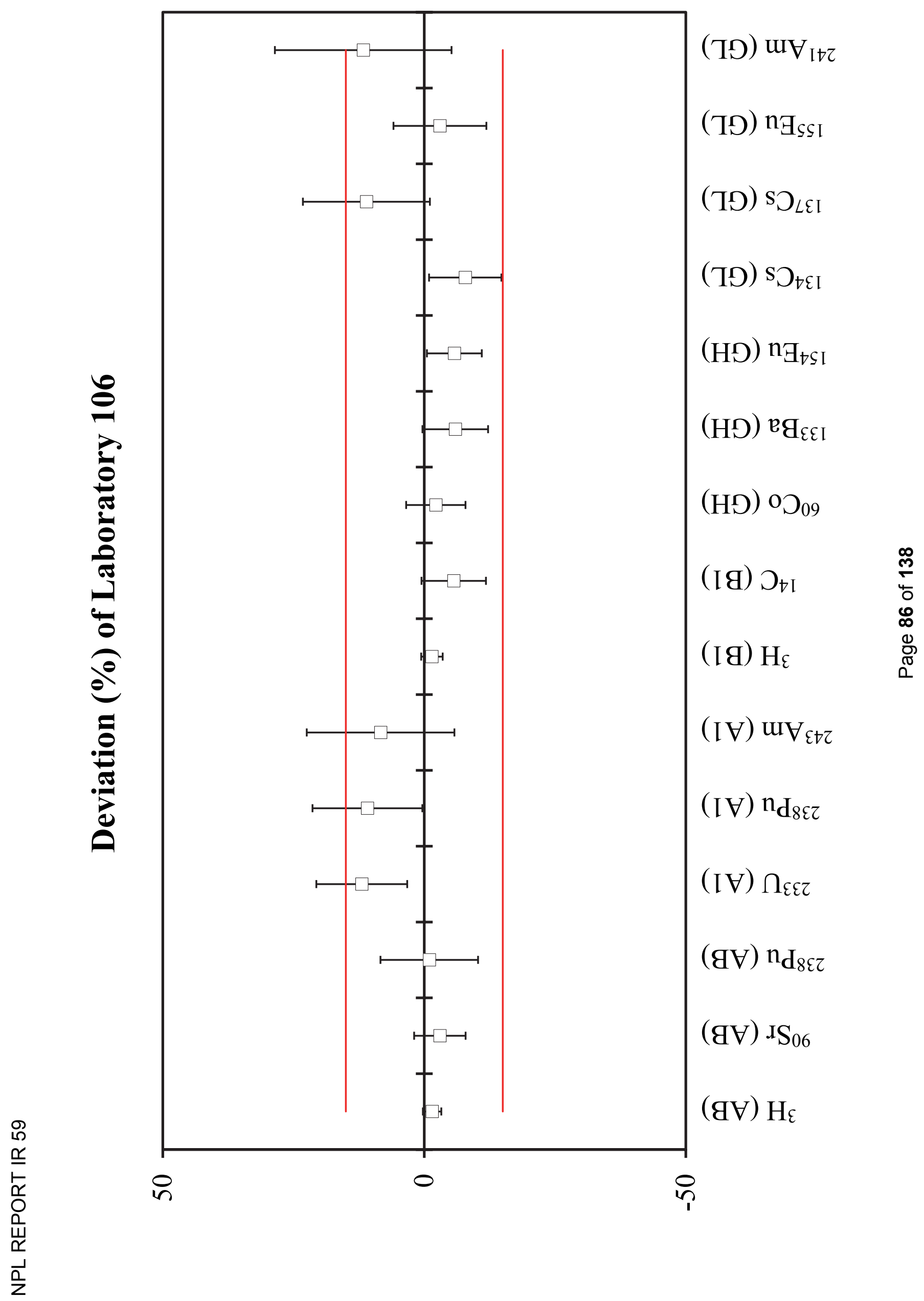




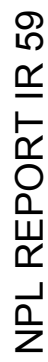

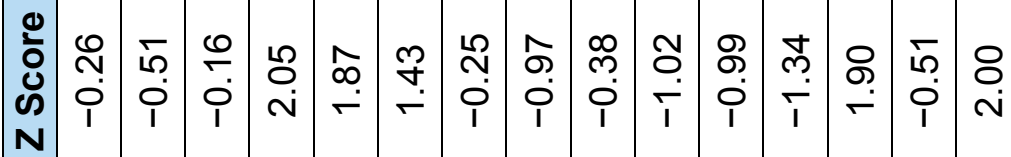

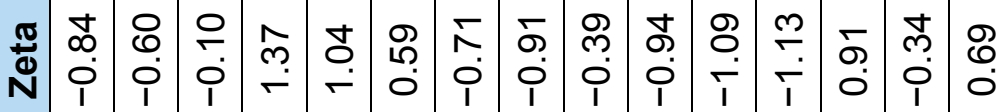

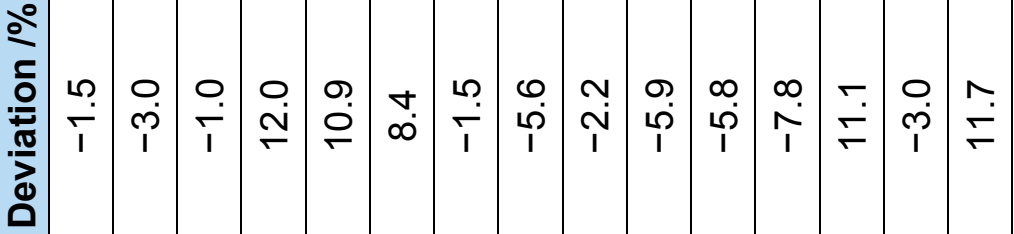

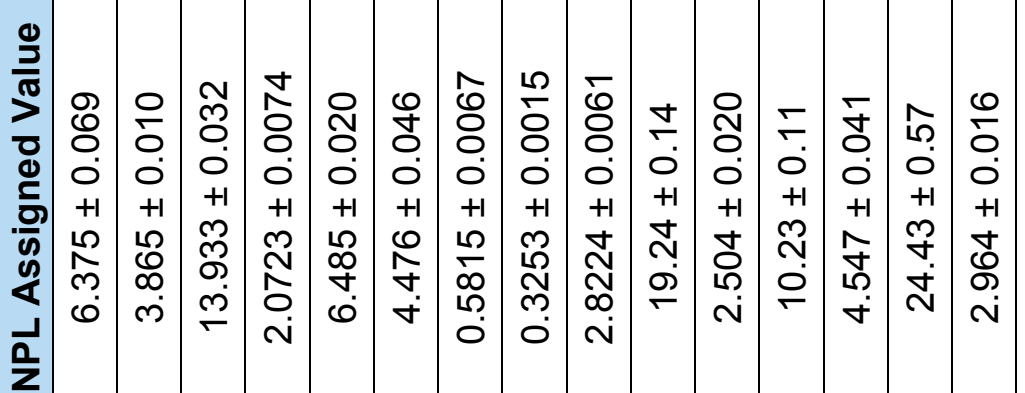

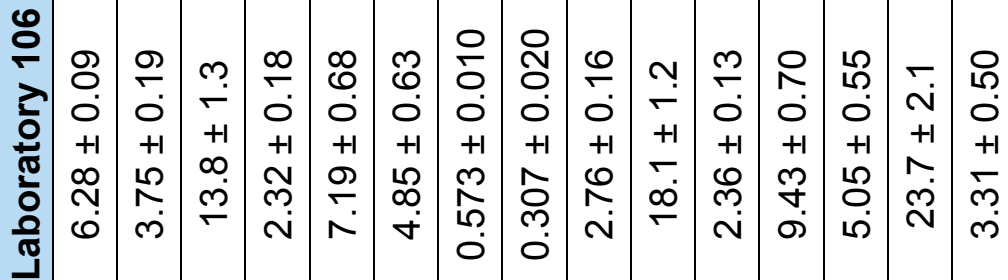

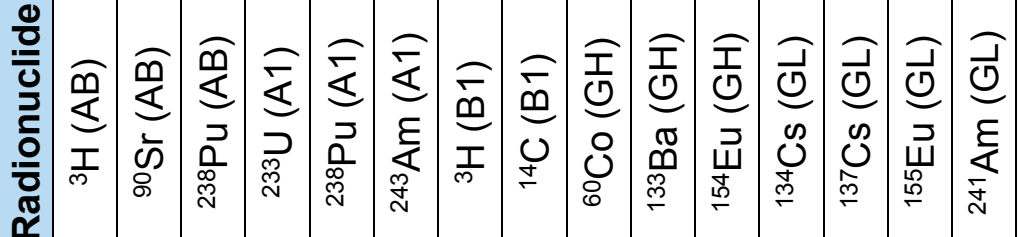




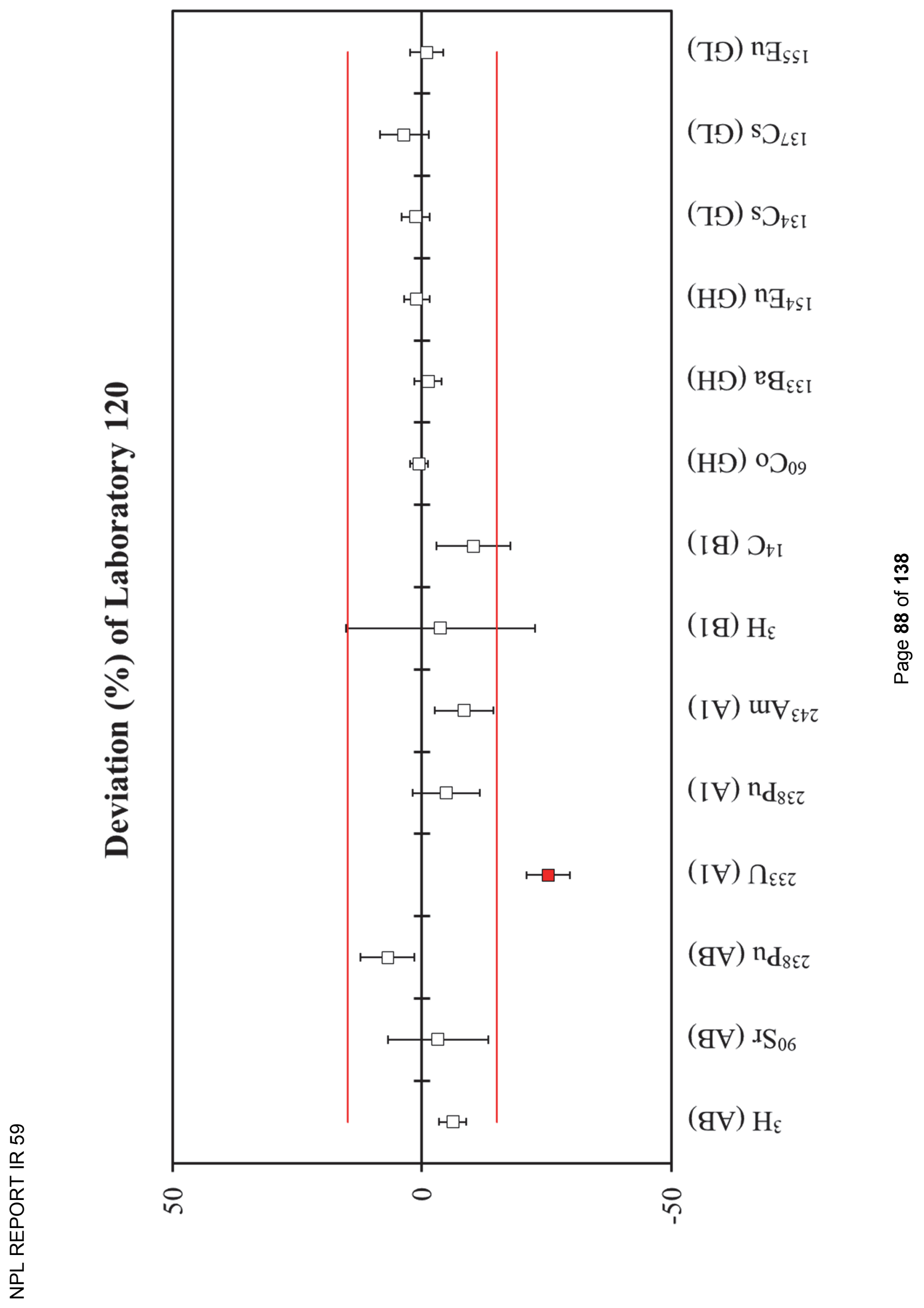




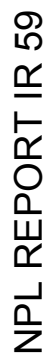

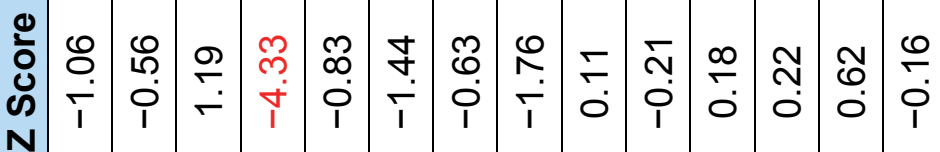

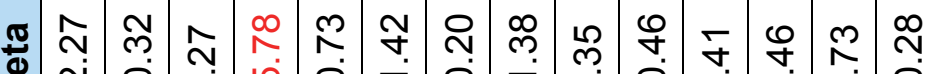
†

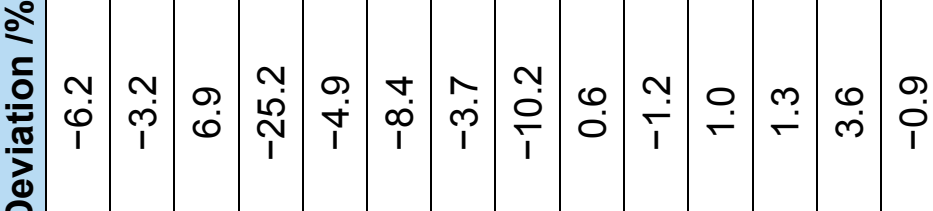

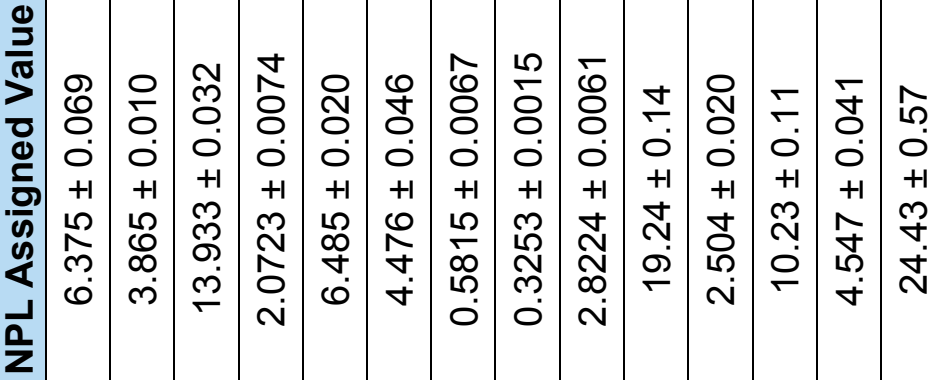

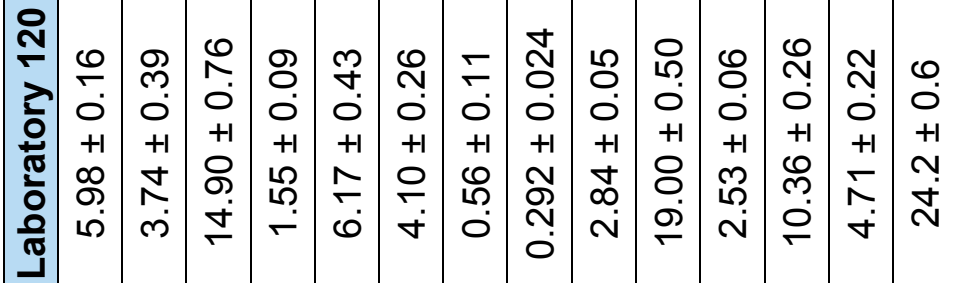

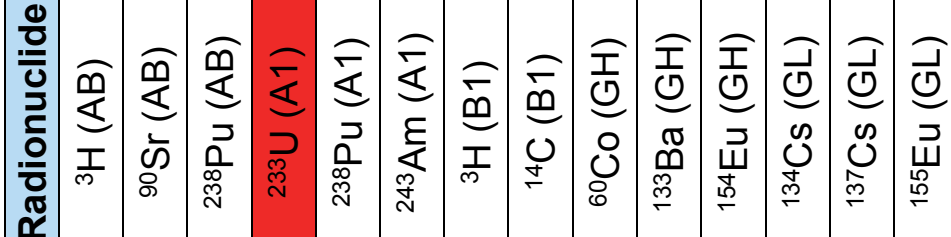




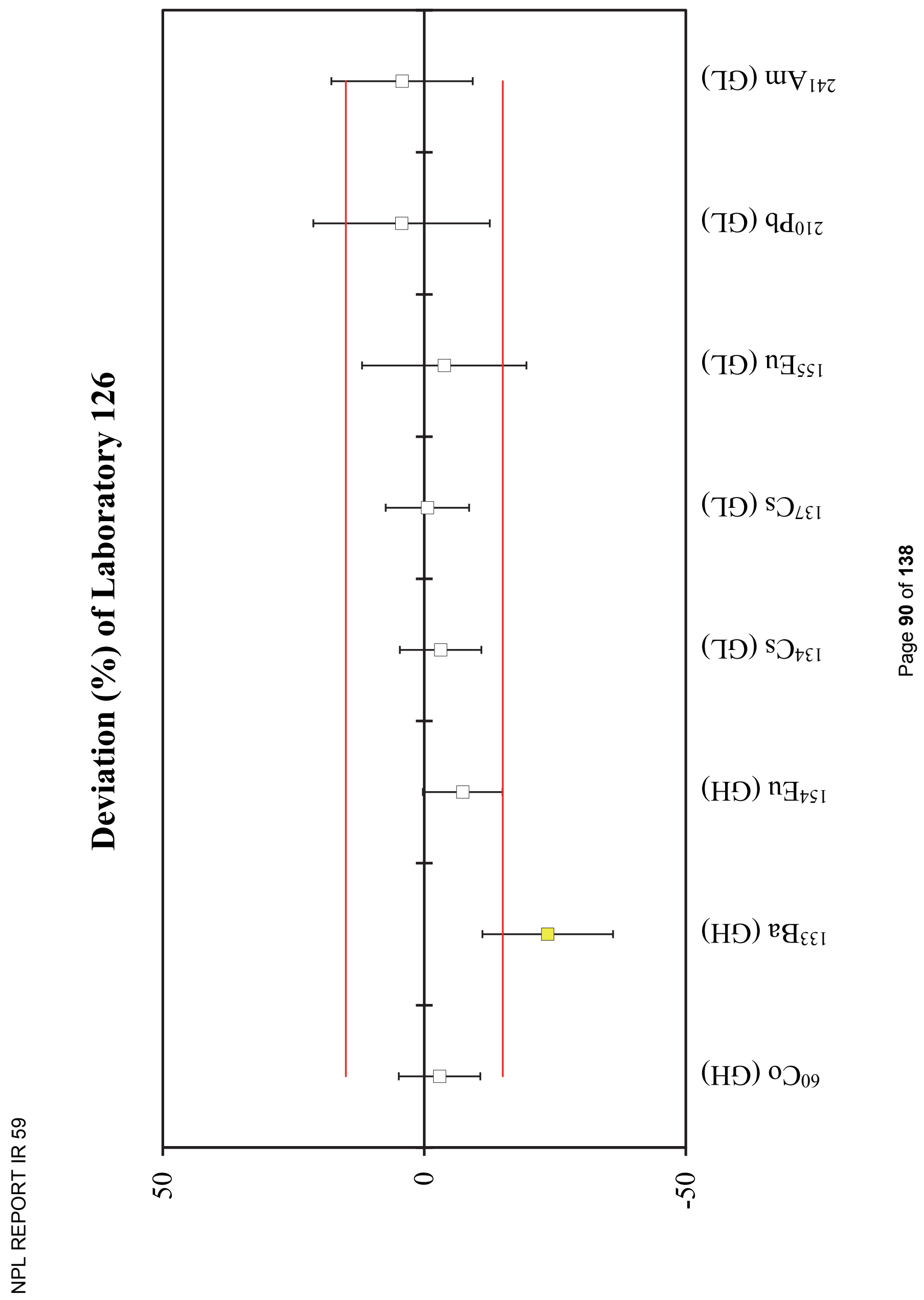




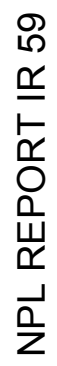

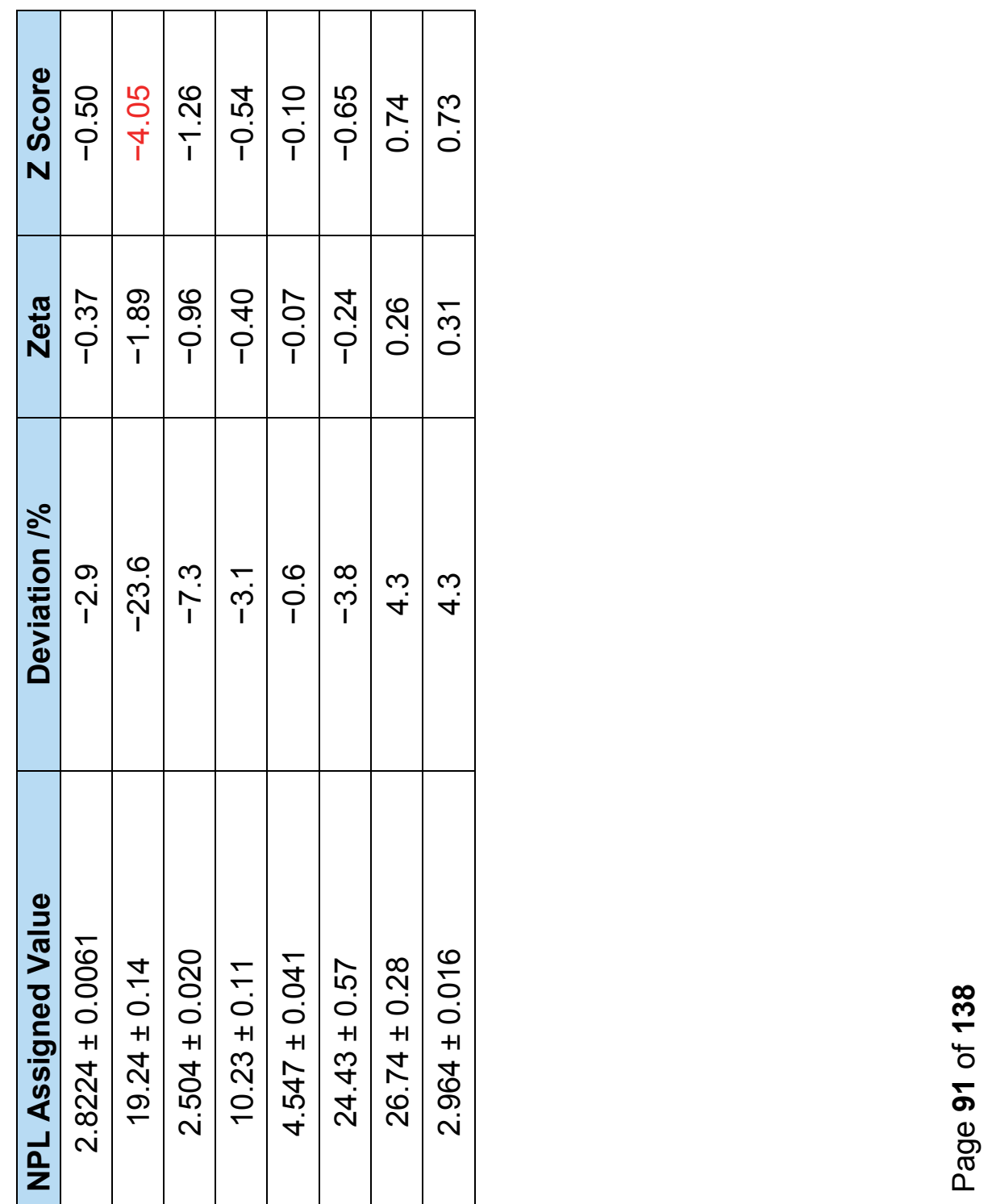

ำ

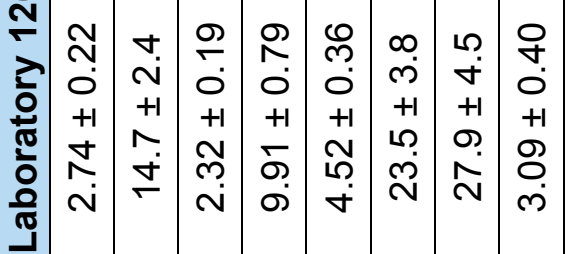

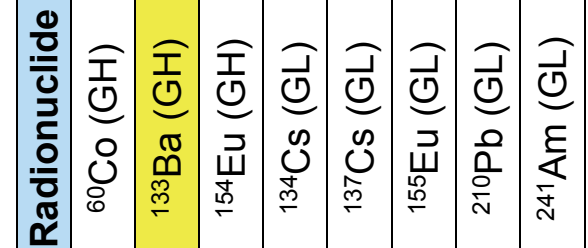




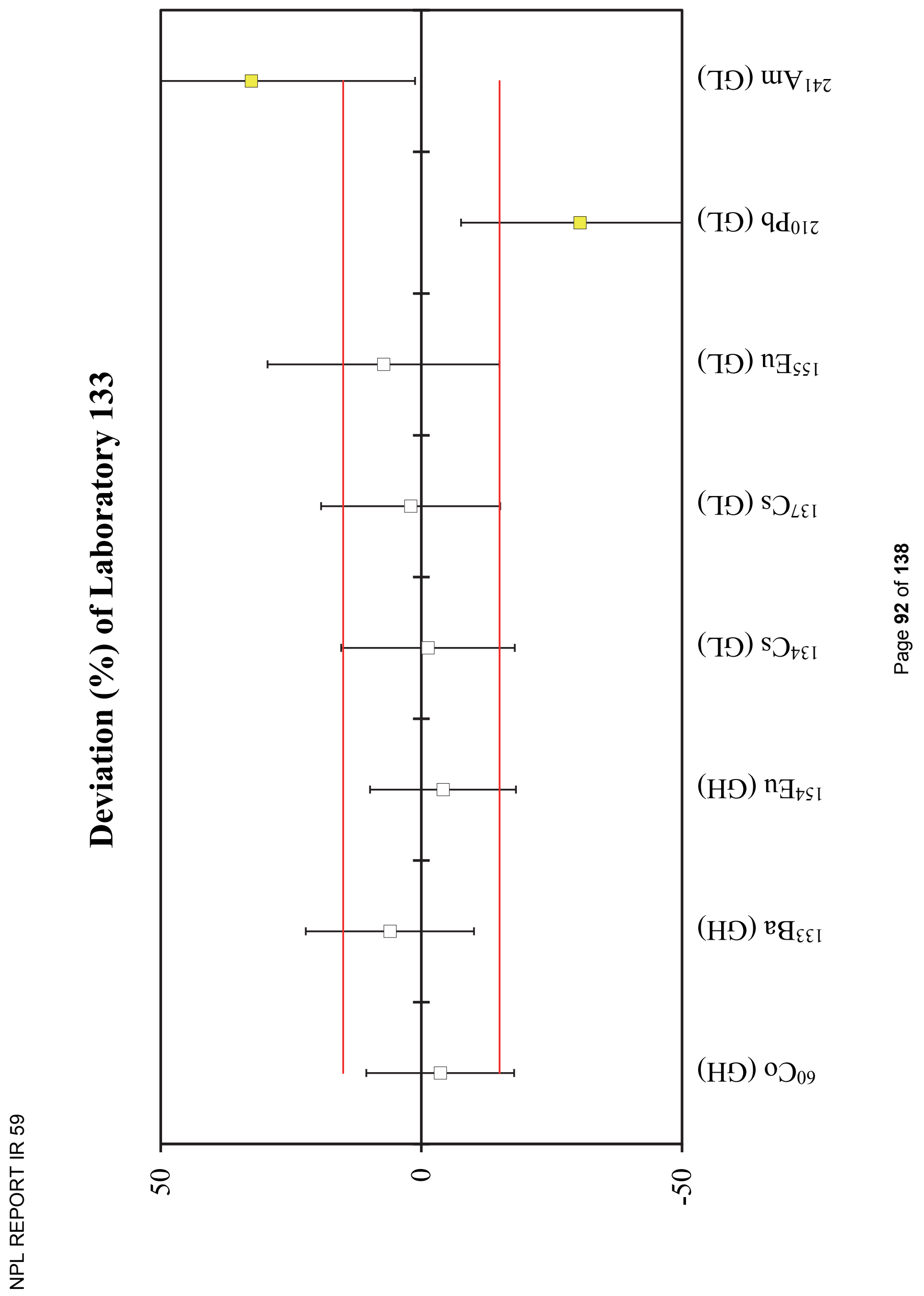




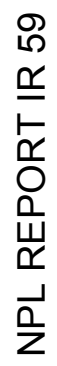

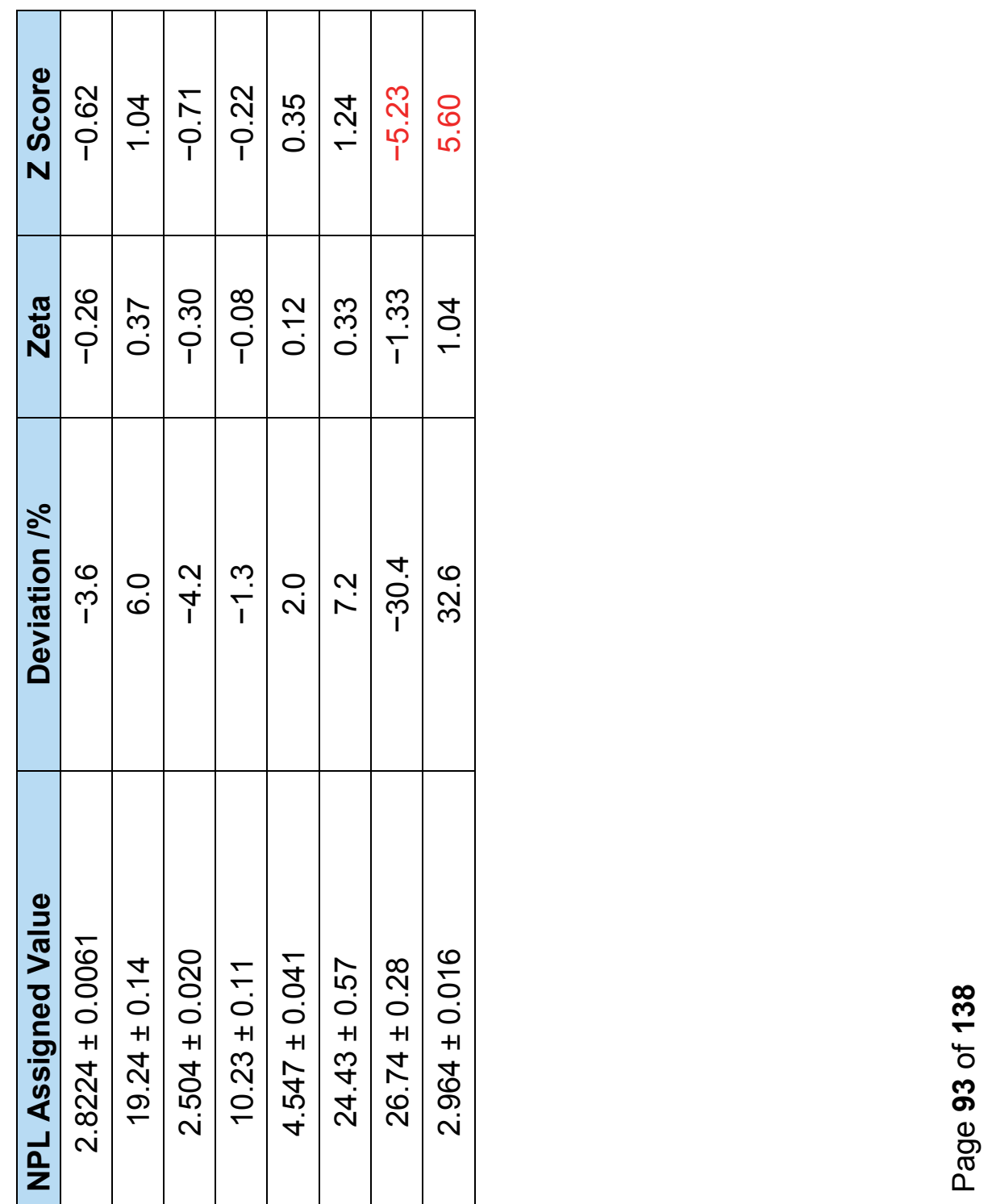

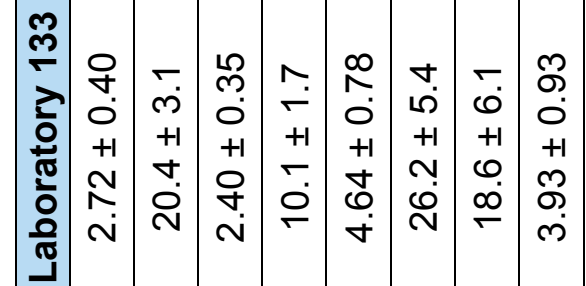

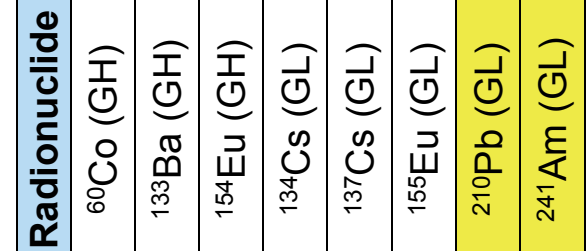




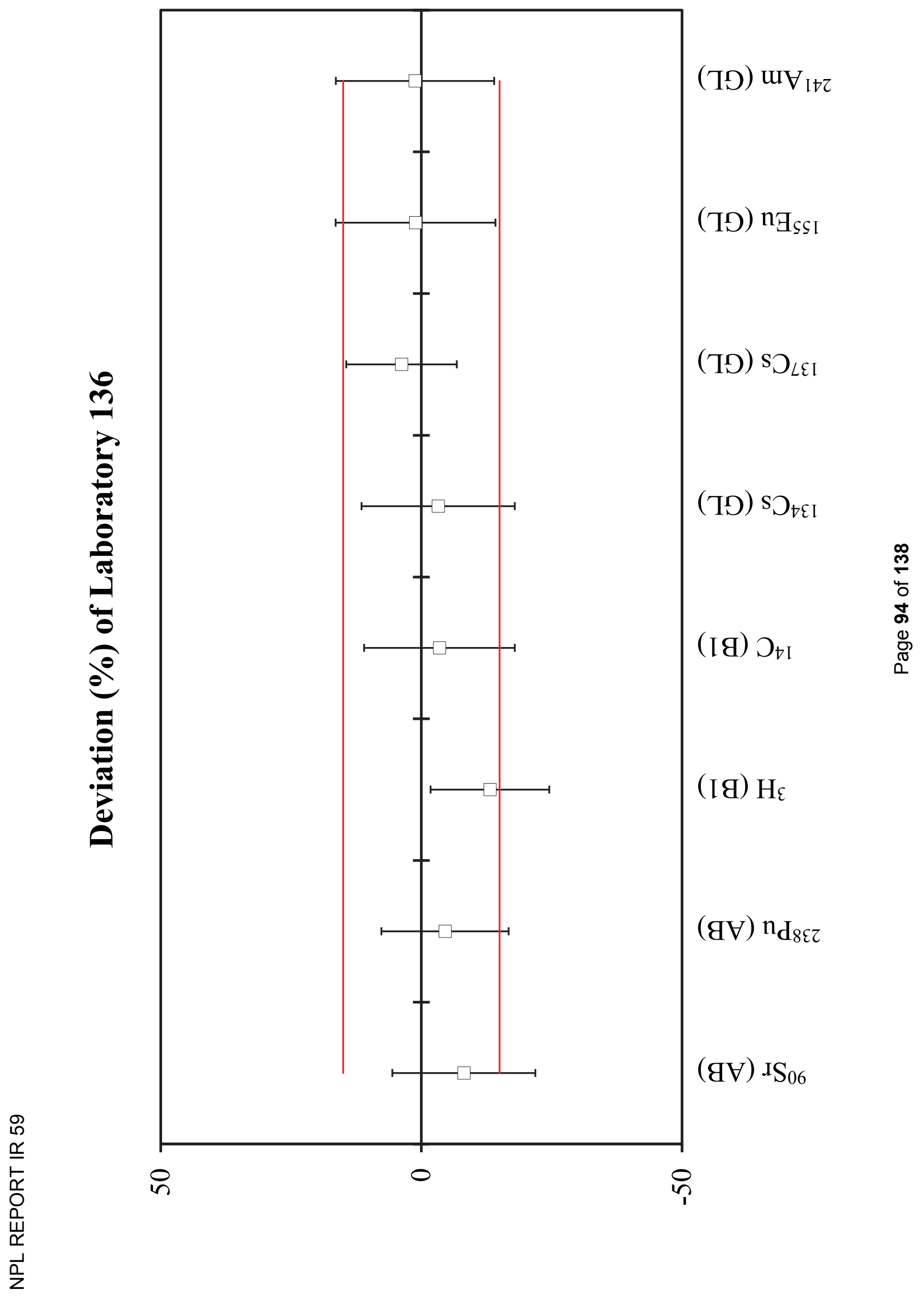




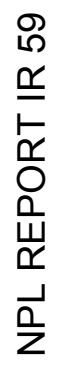

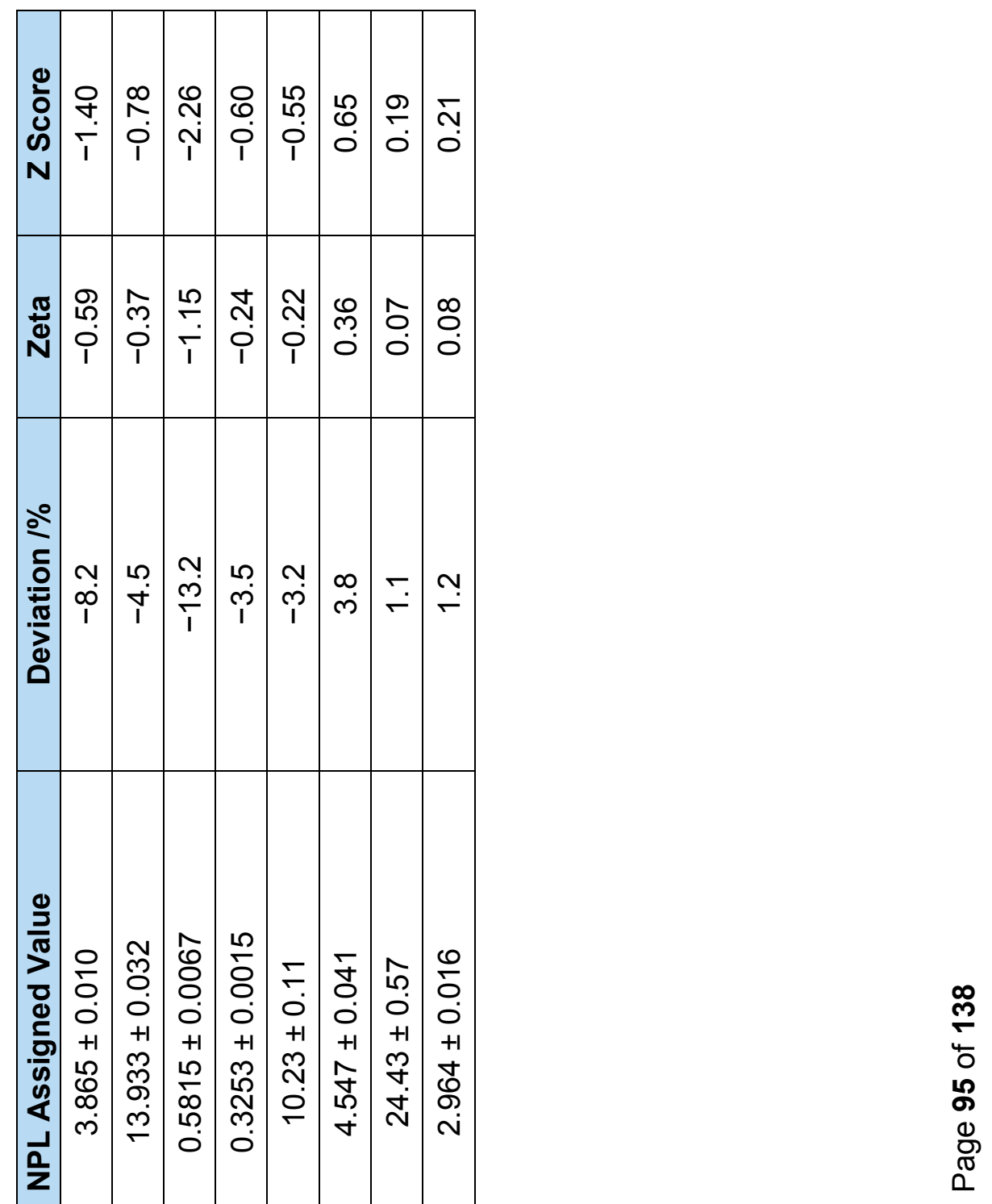

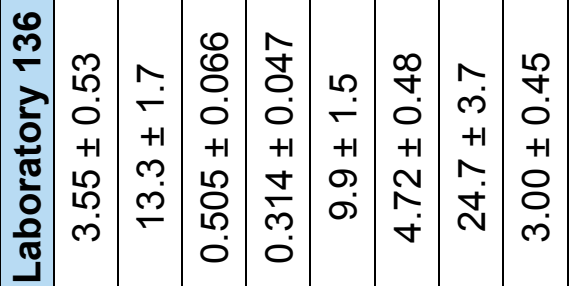

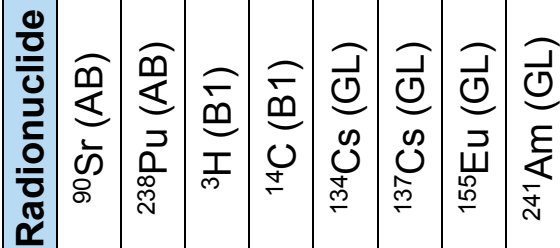




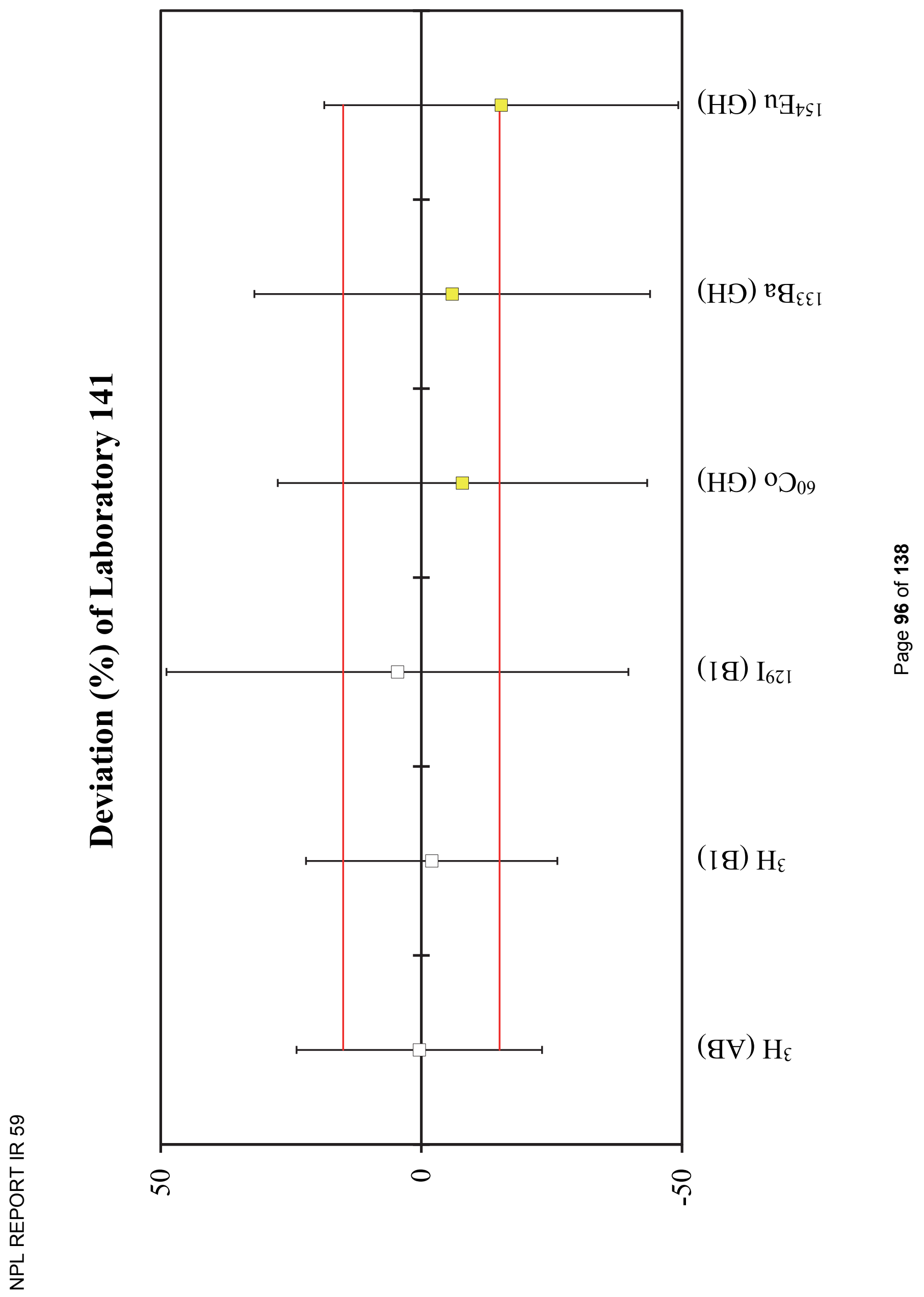




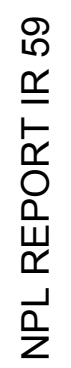

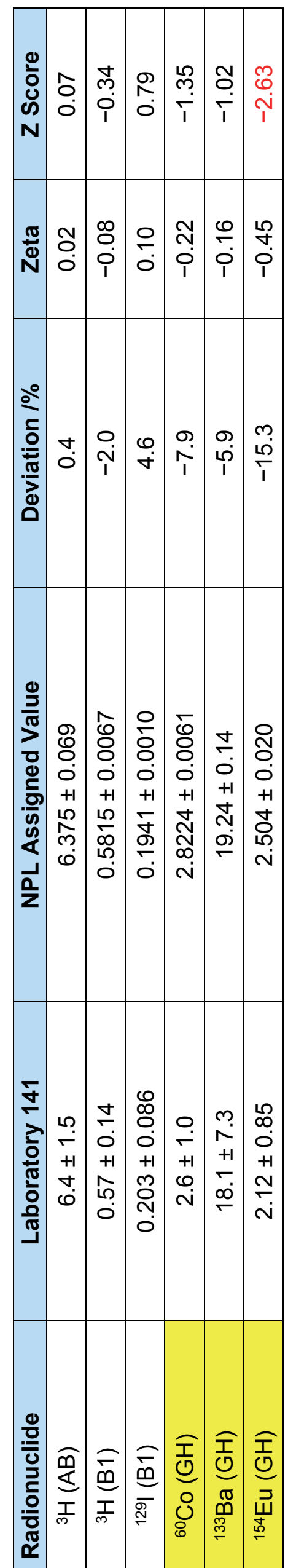

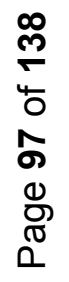




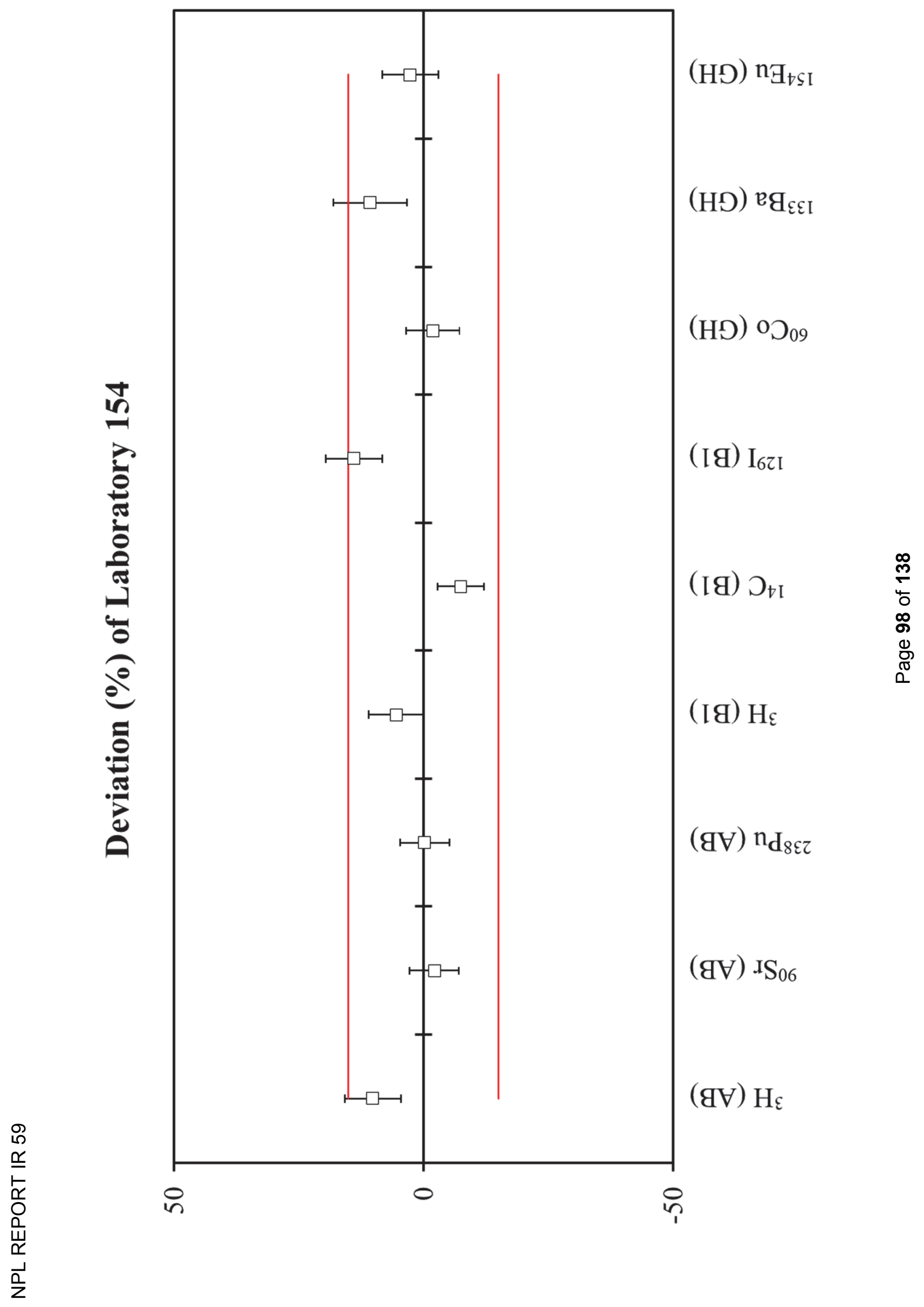




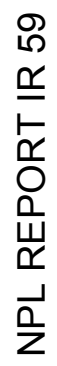

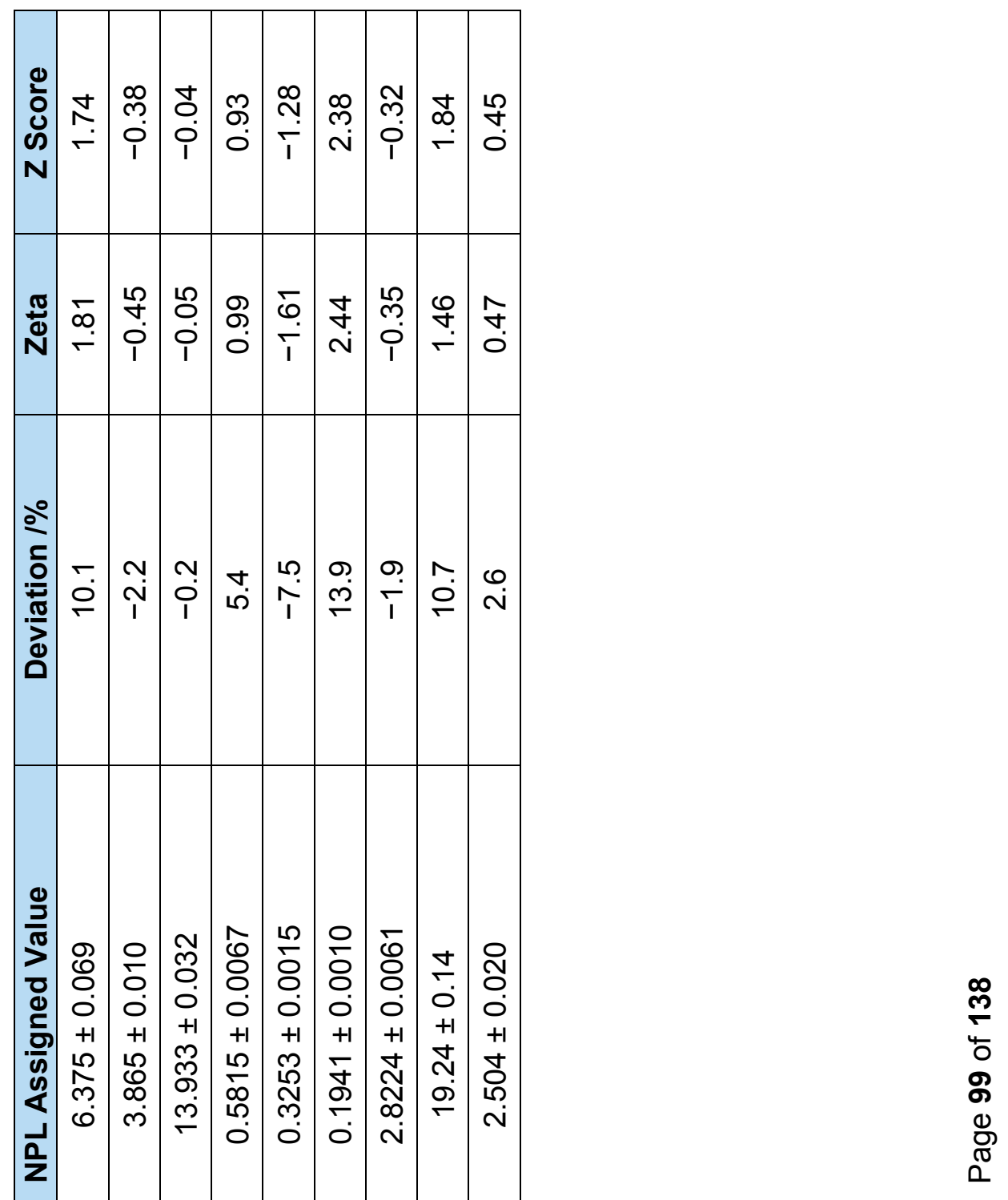

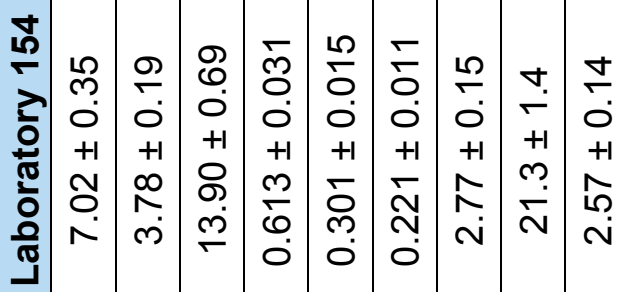

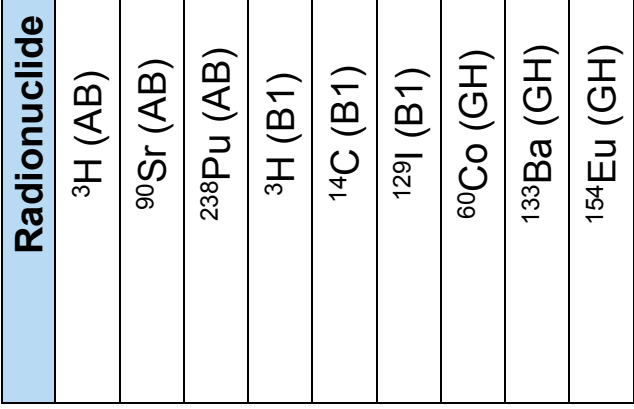




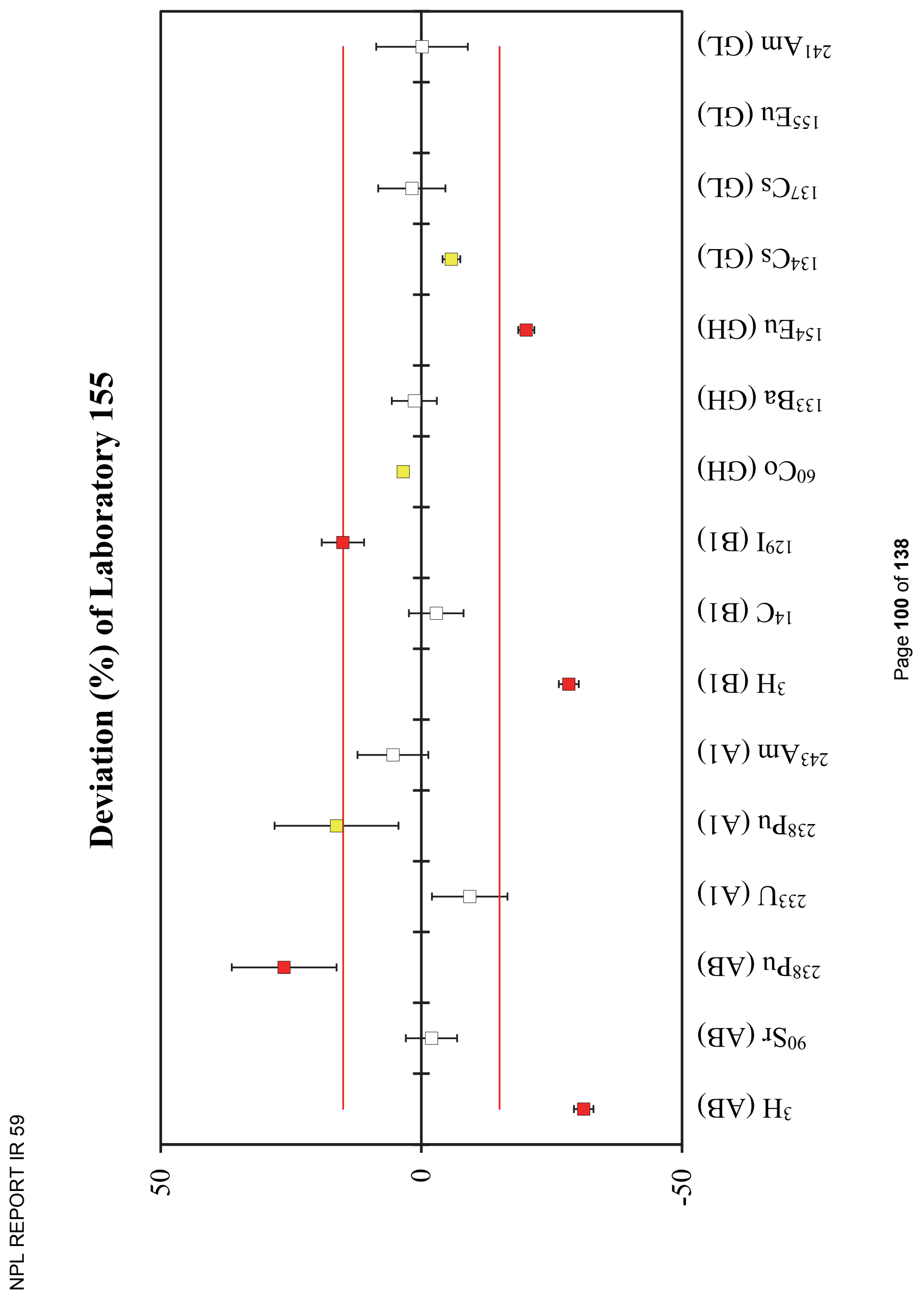




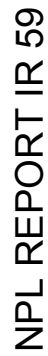

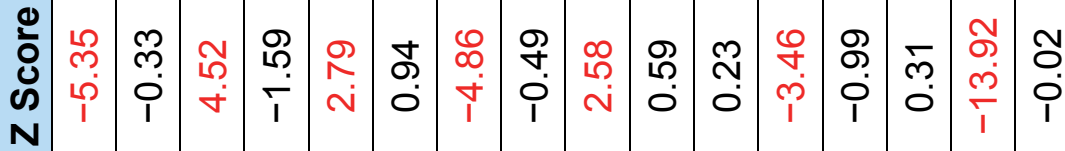

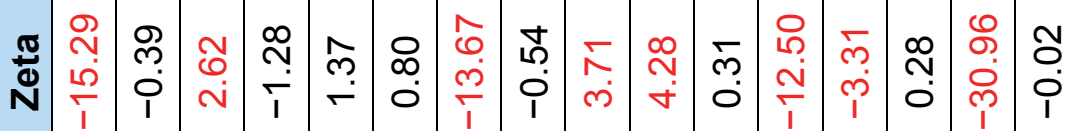

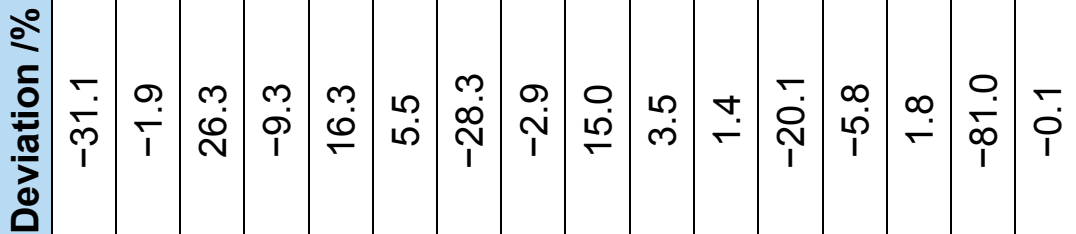

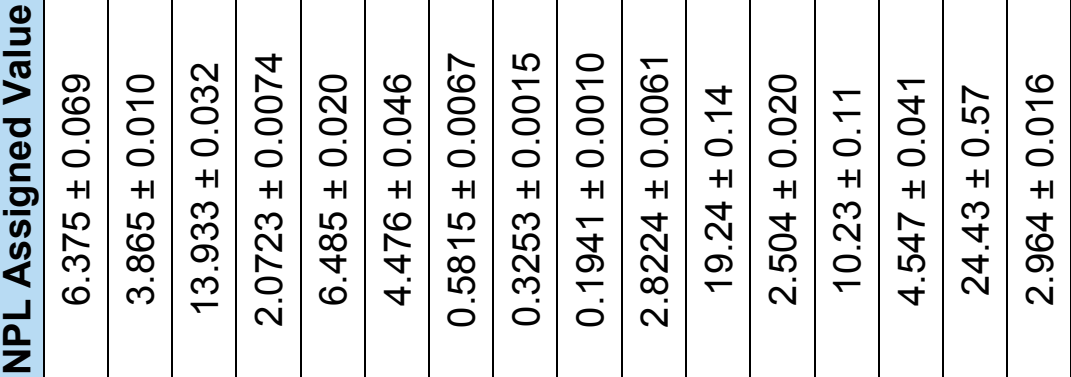

$\stackrel{\infty}{2}$
$\frac{4}{0}$
$\frac{0}{0}$
$\frac{0}{0}$
$\frac{0}{0}$

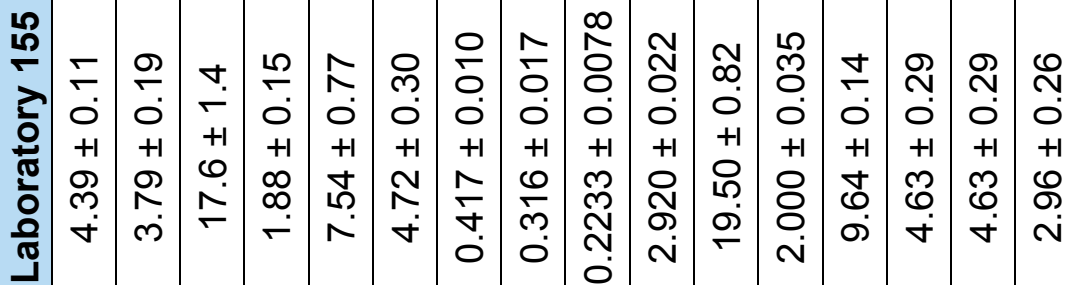

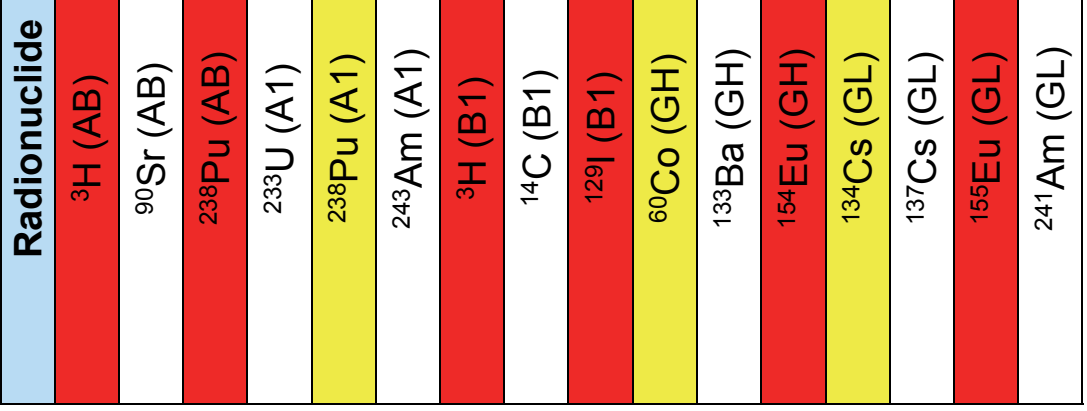




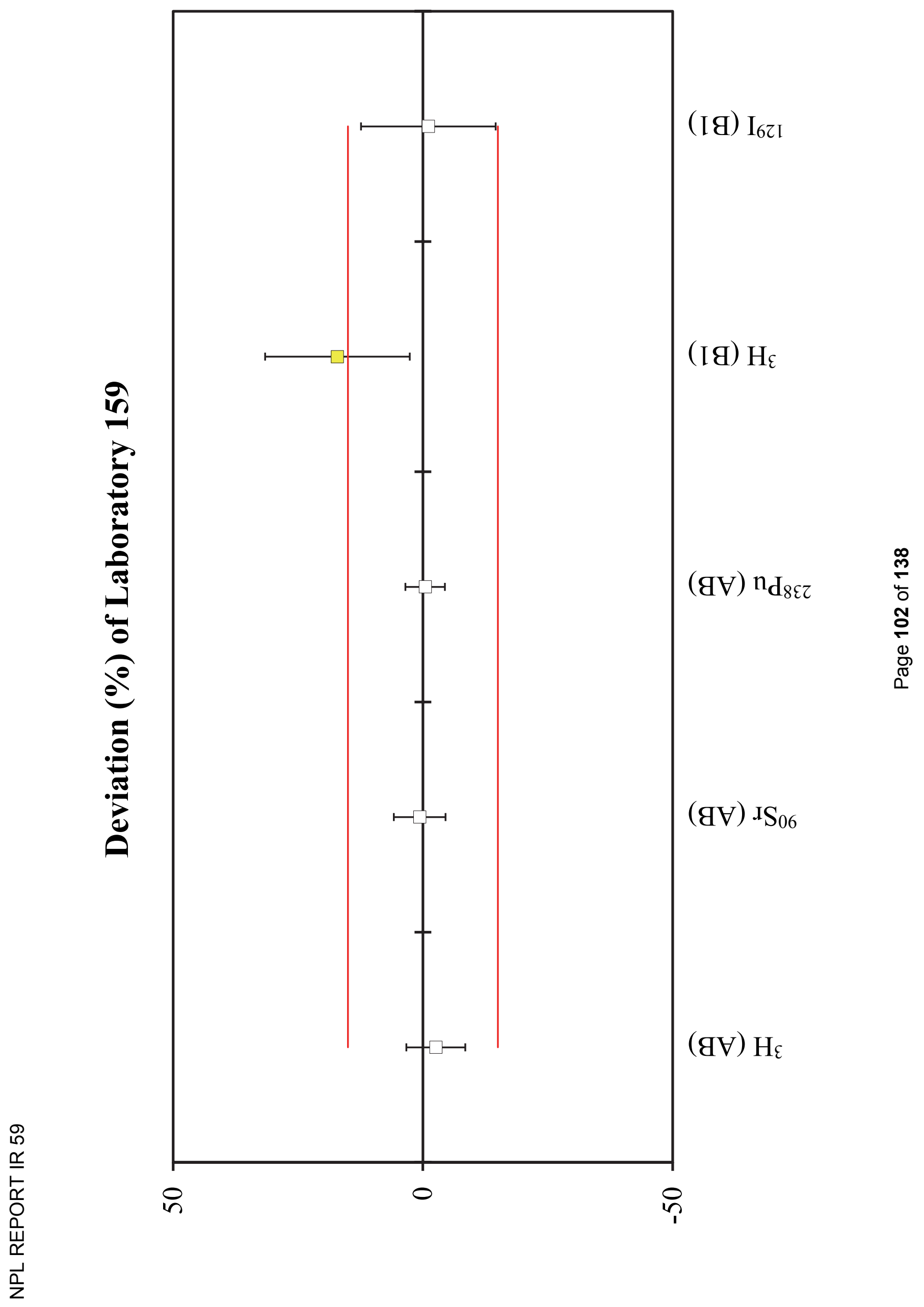




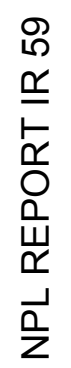

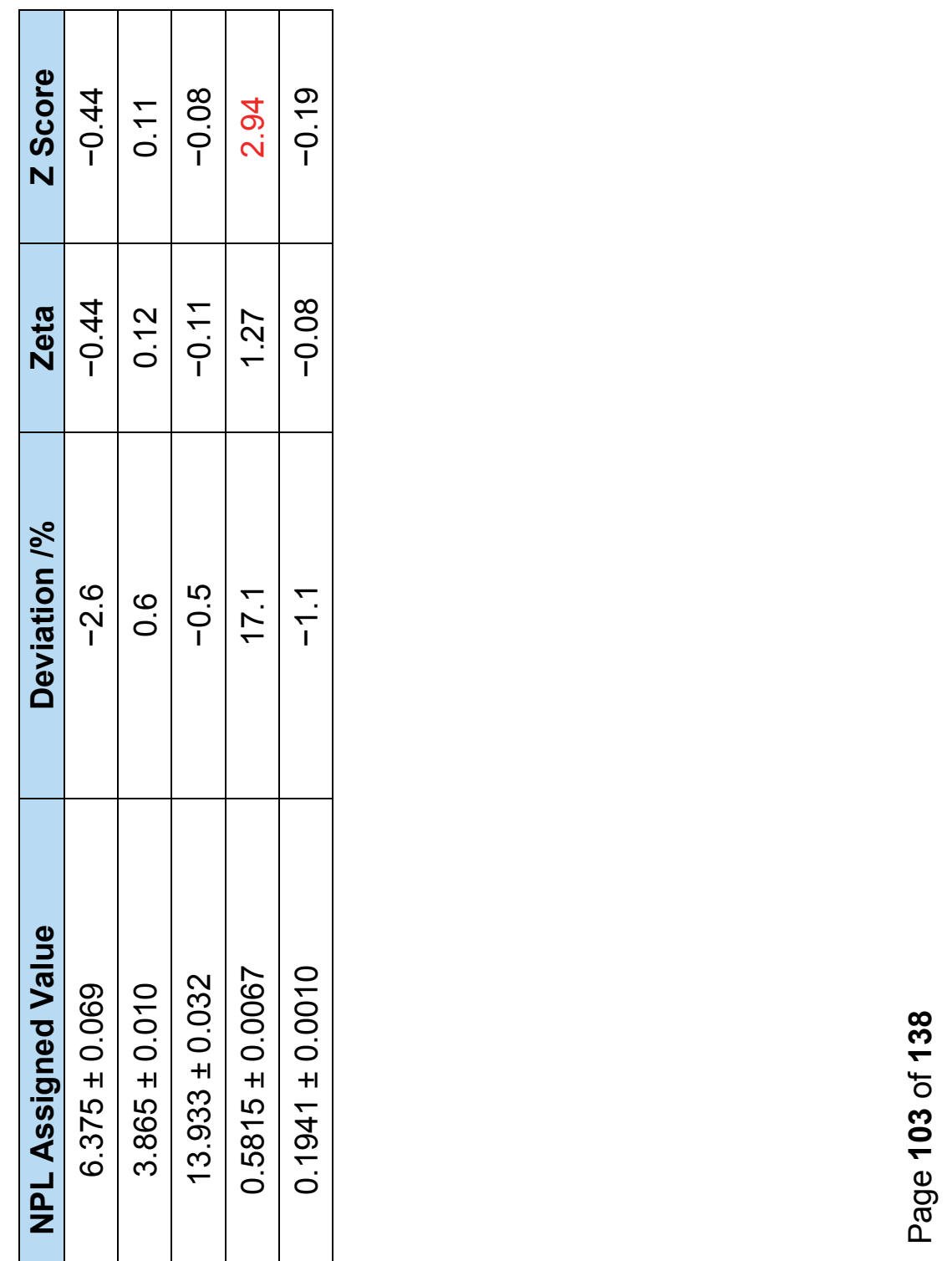

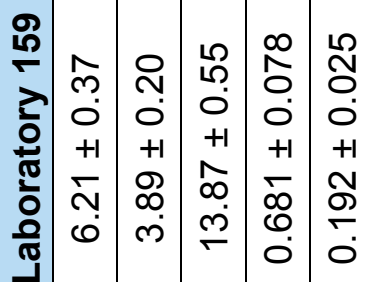

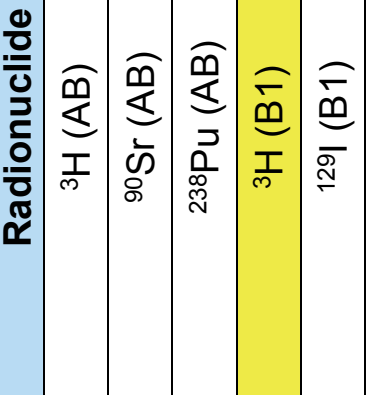




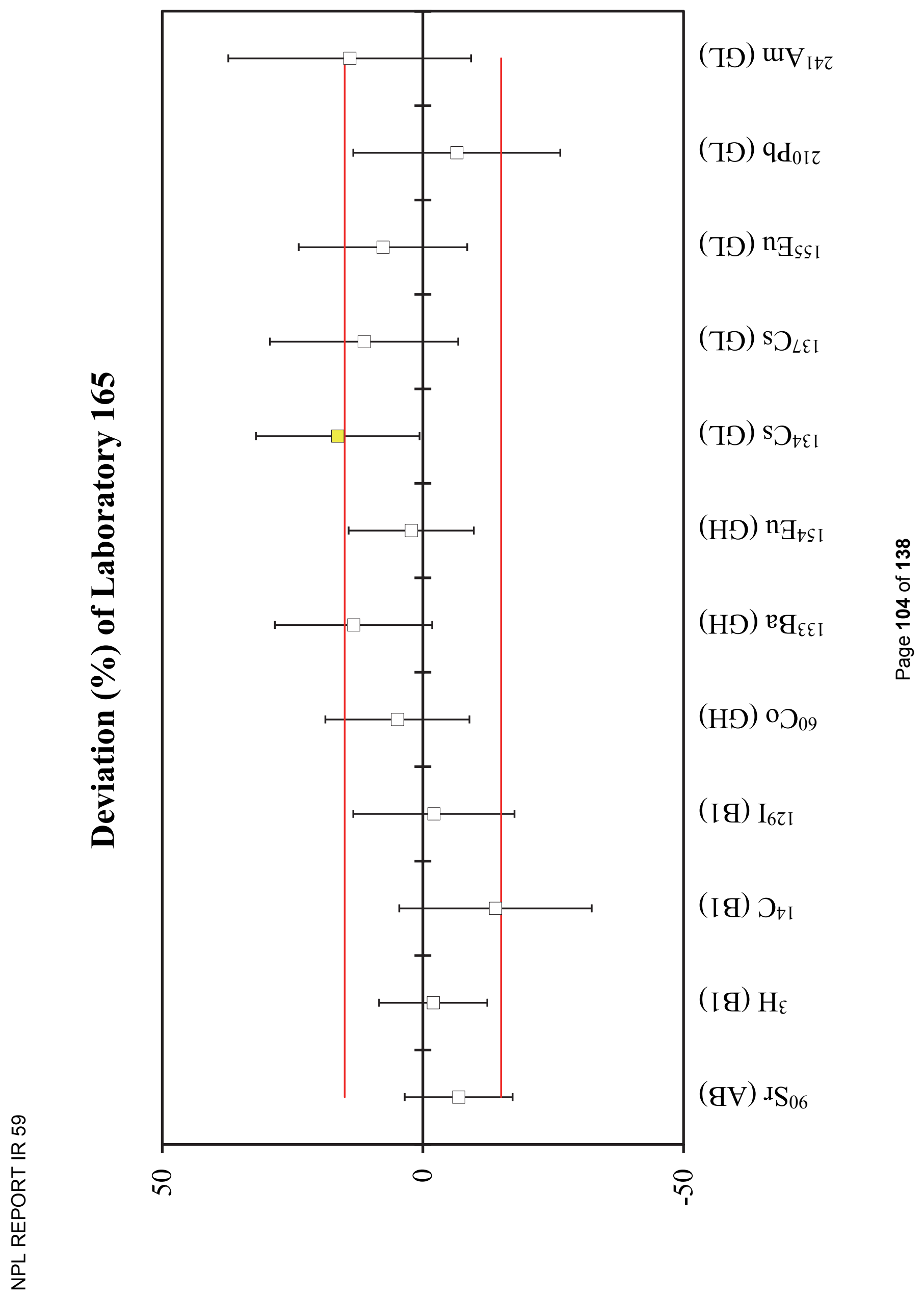




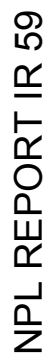

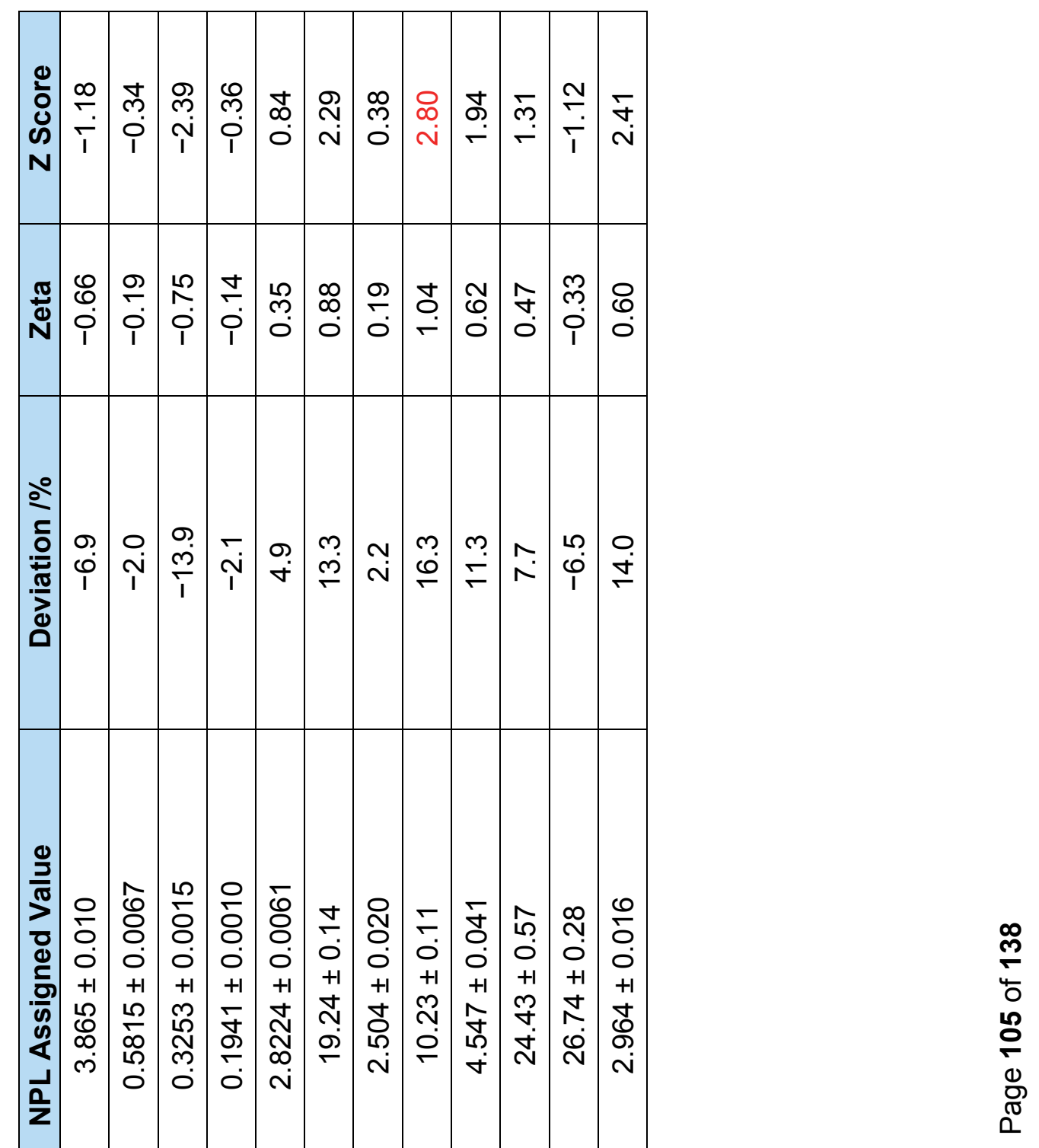

용

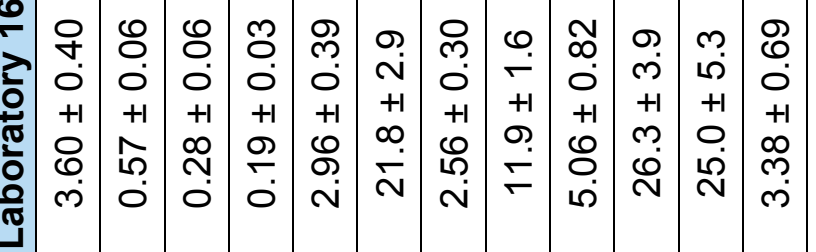




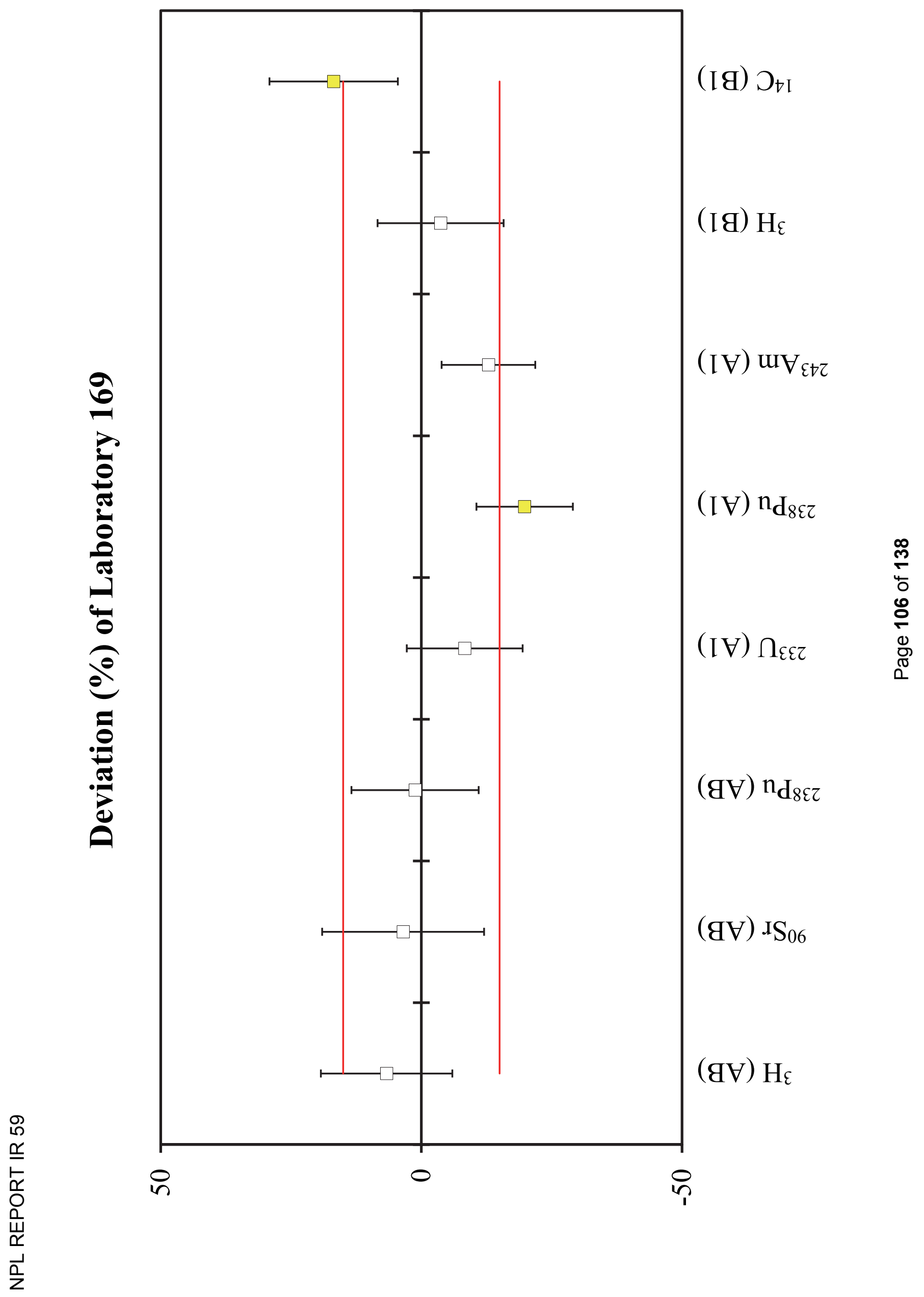




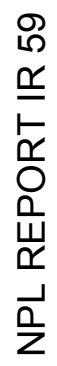

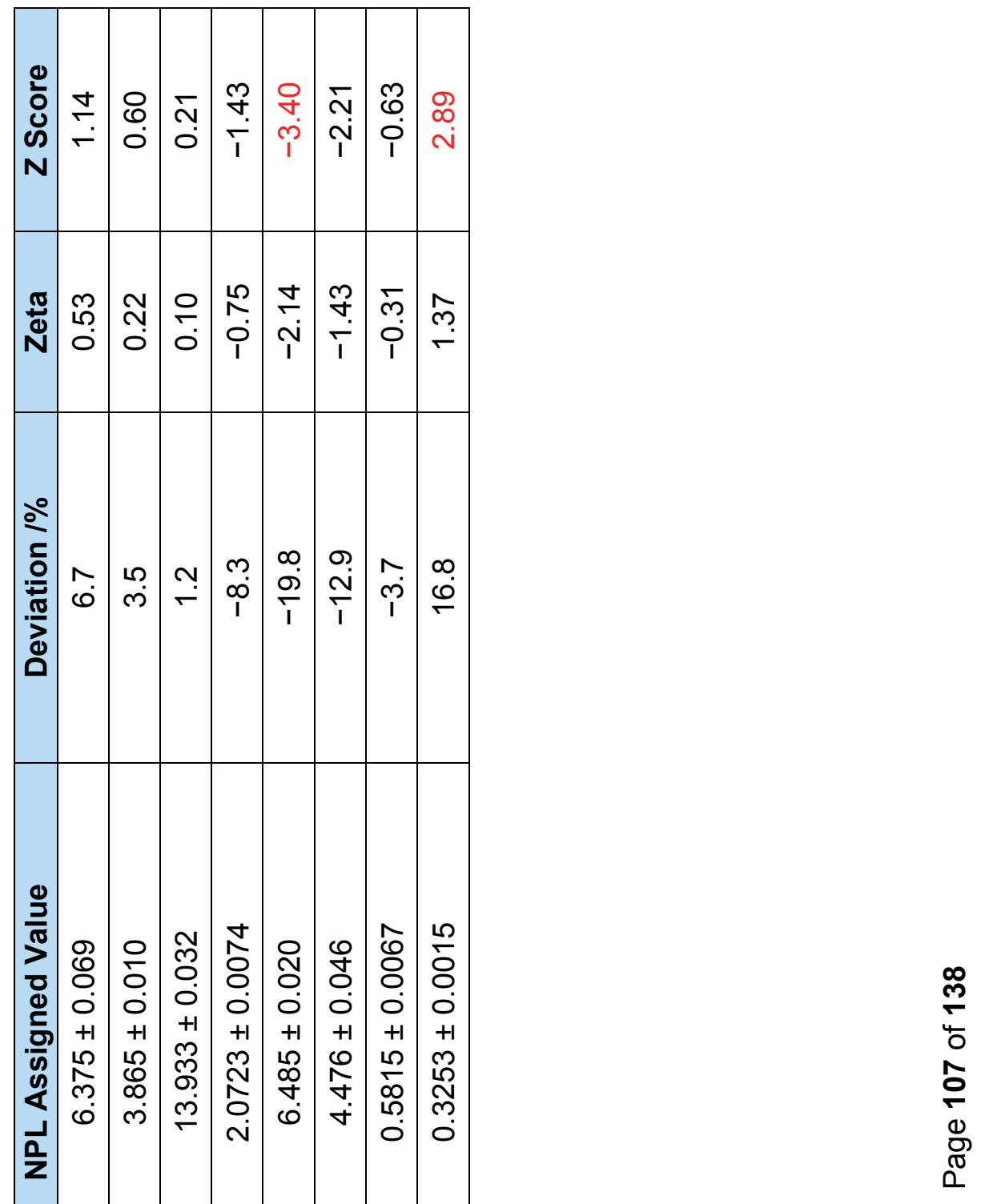

음

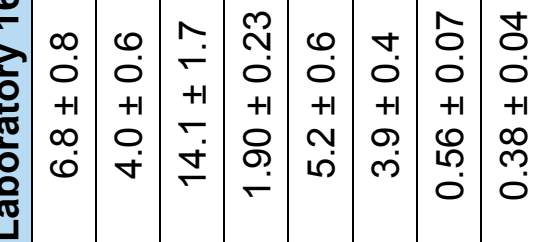

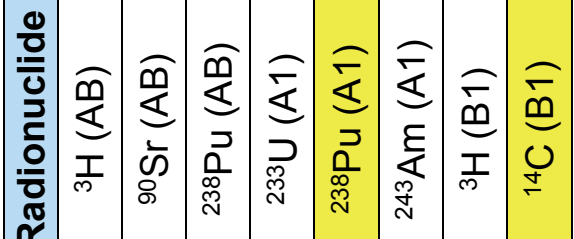




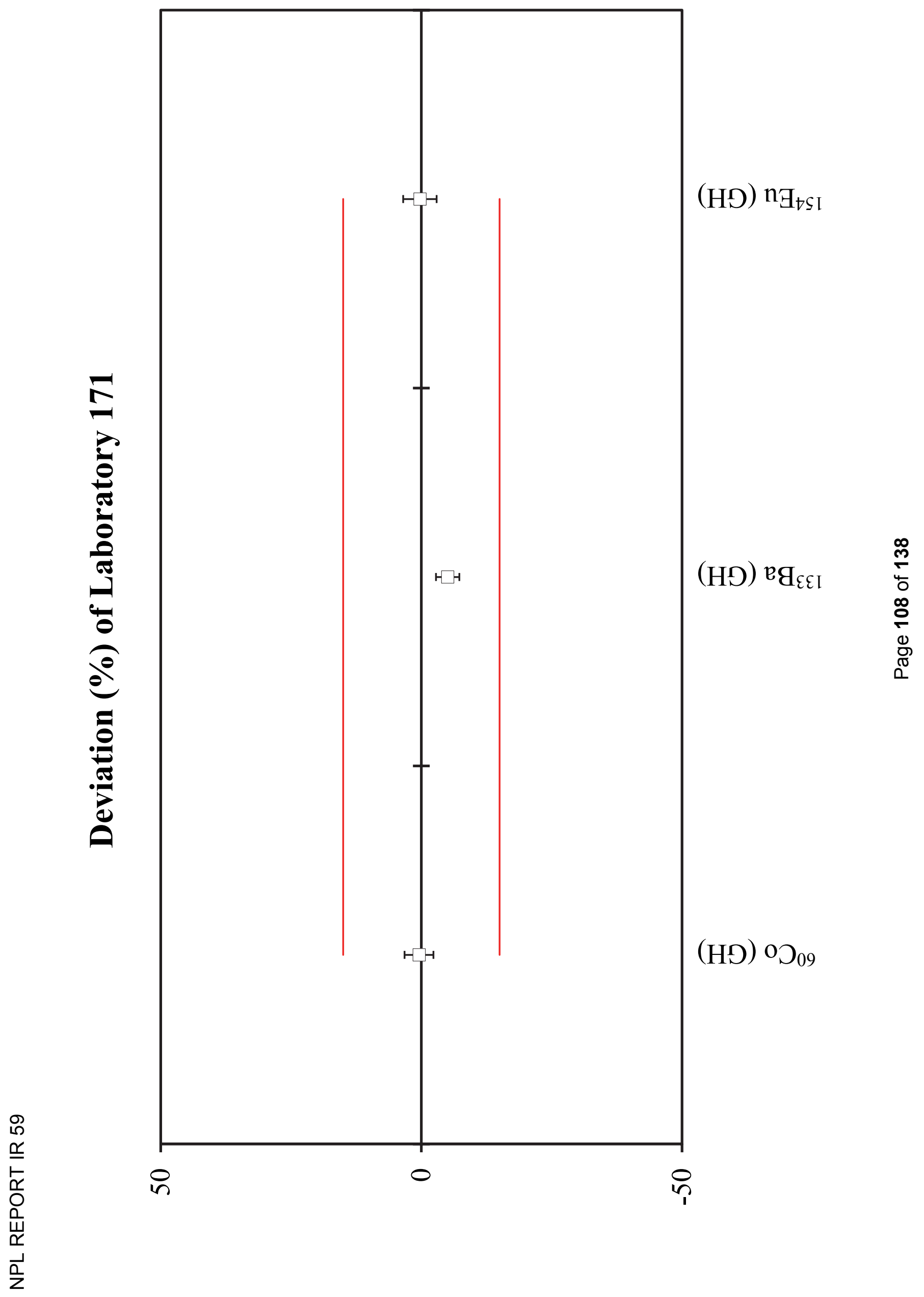




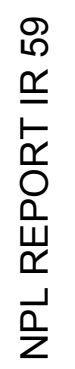

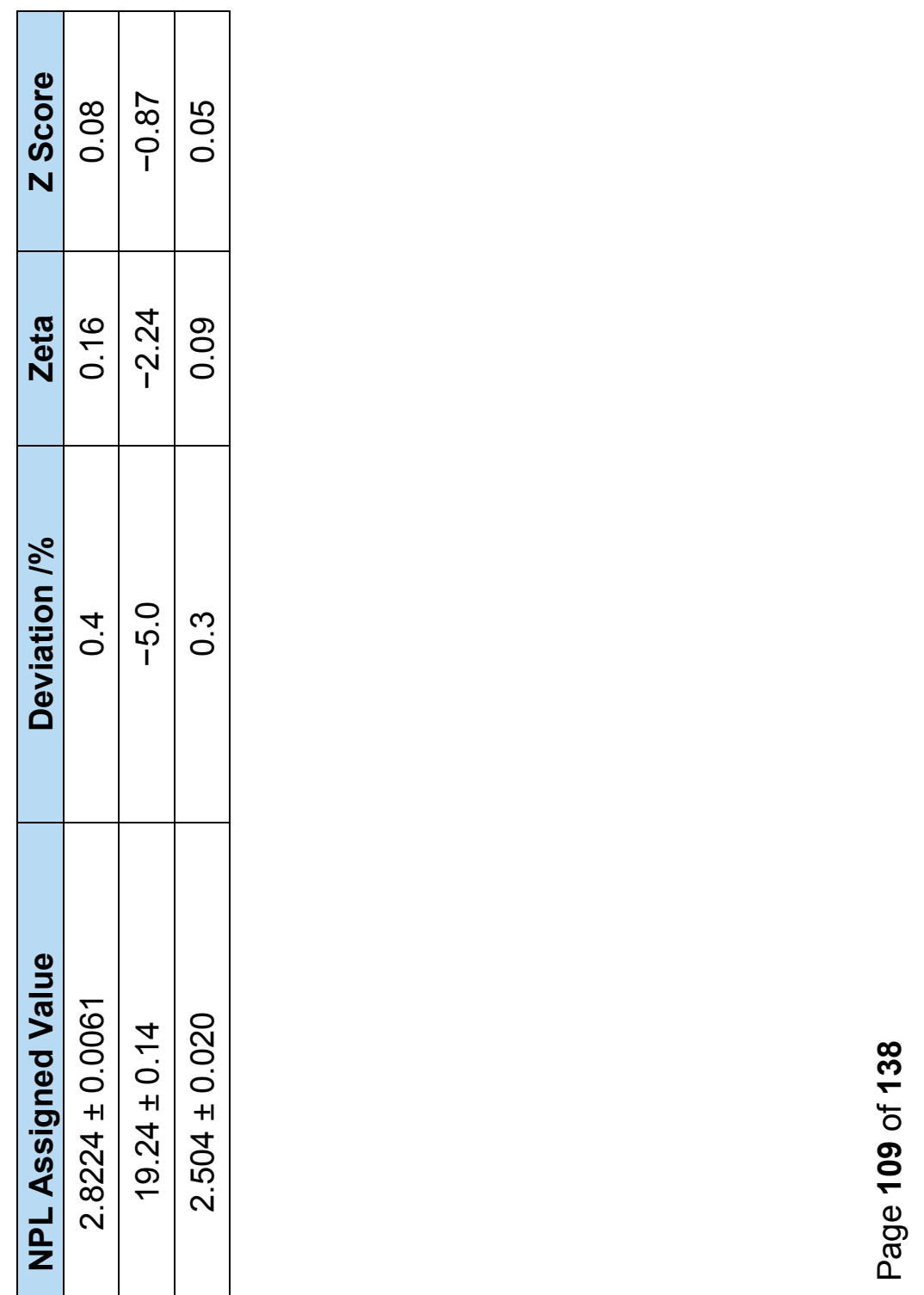

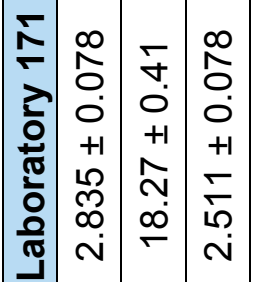

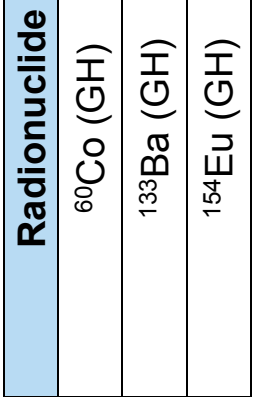




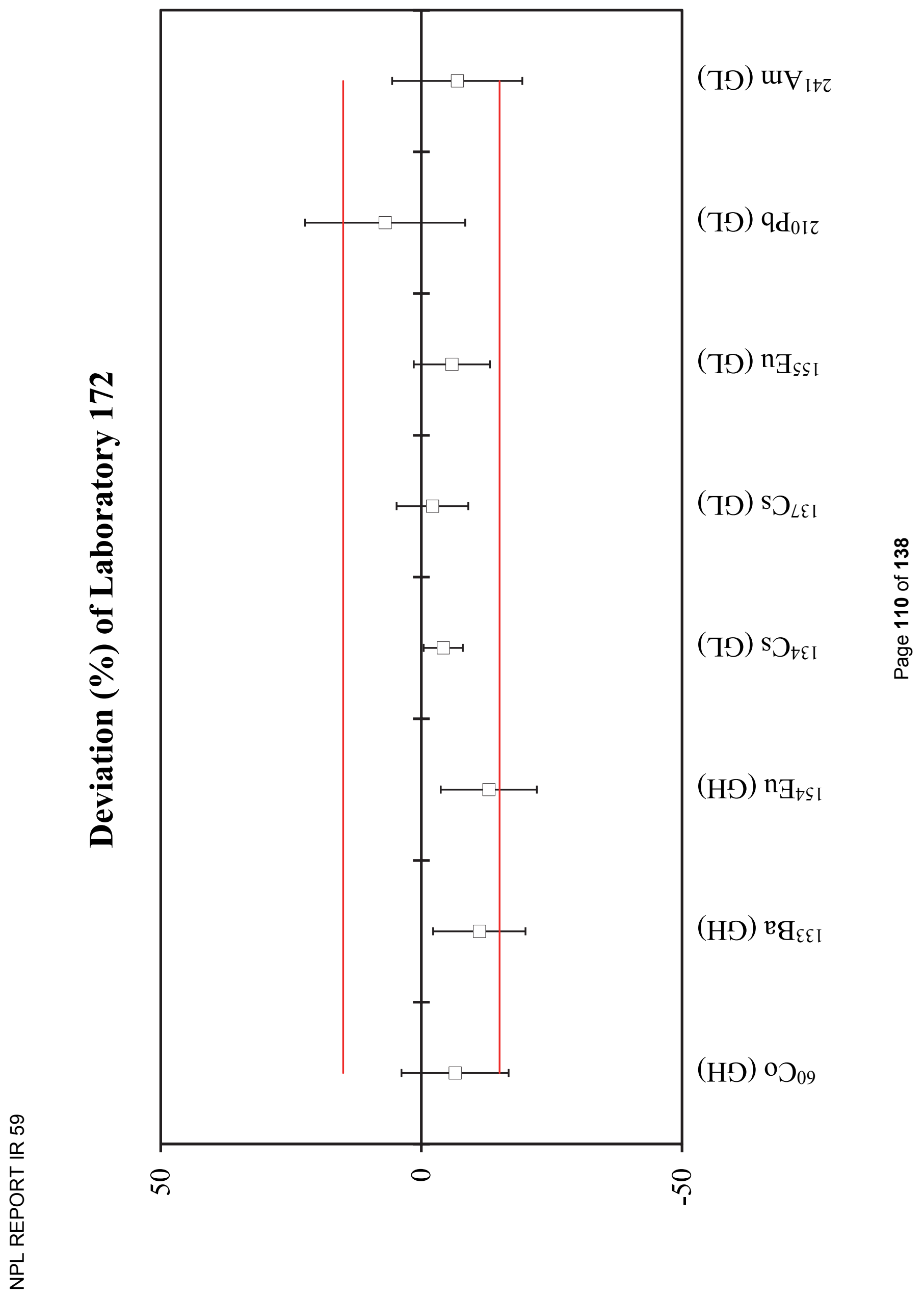




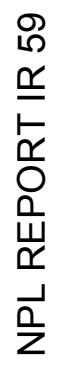

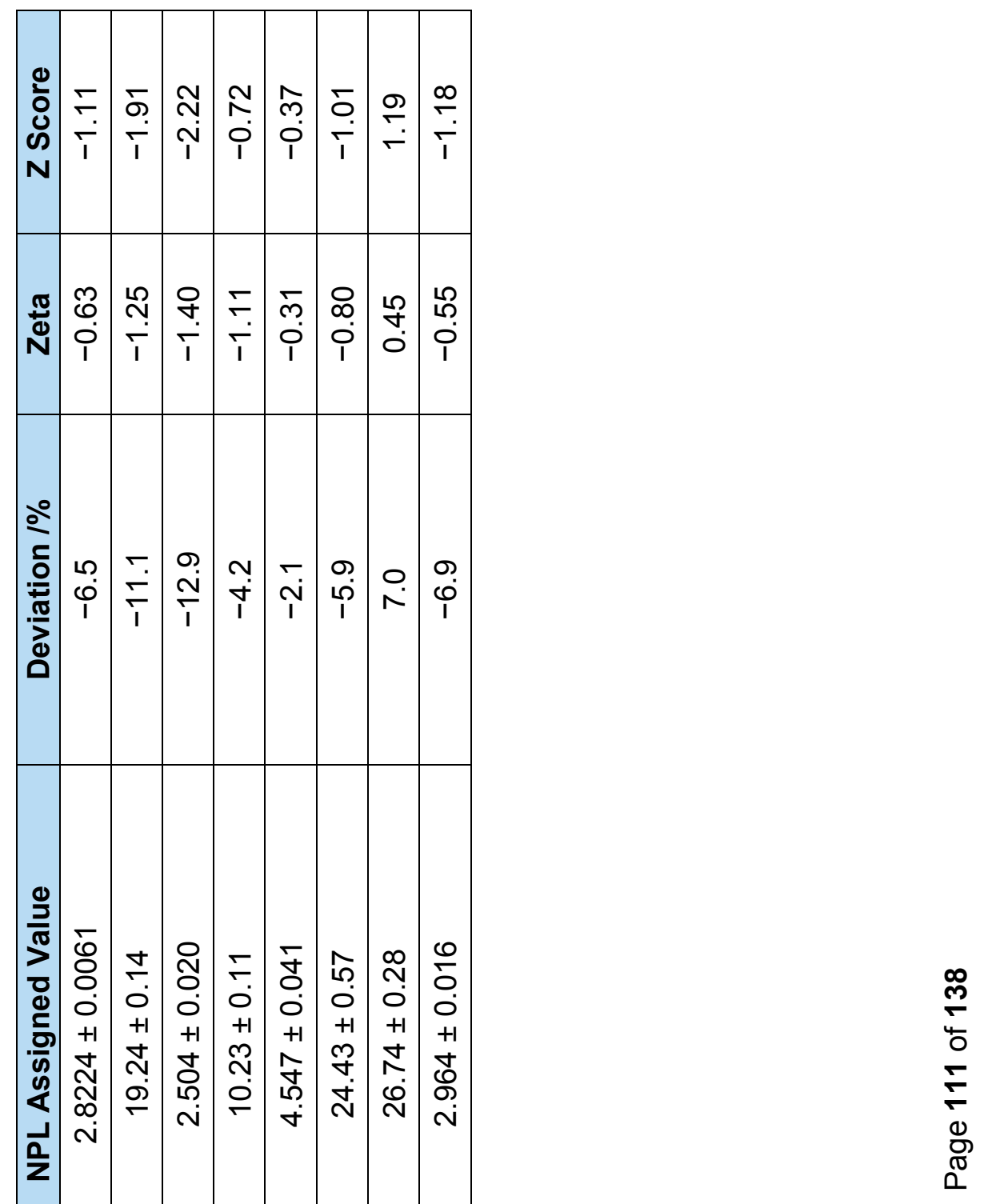

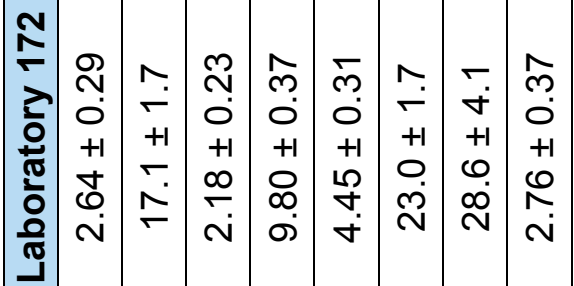

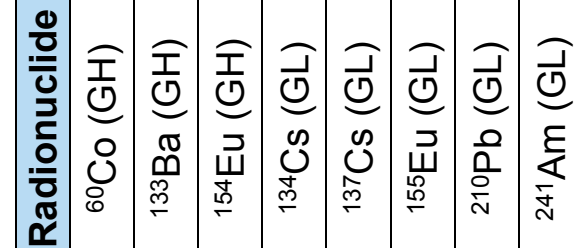




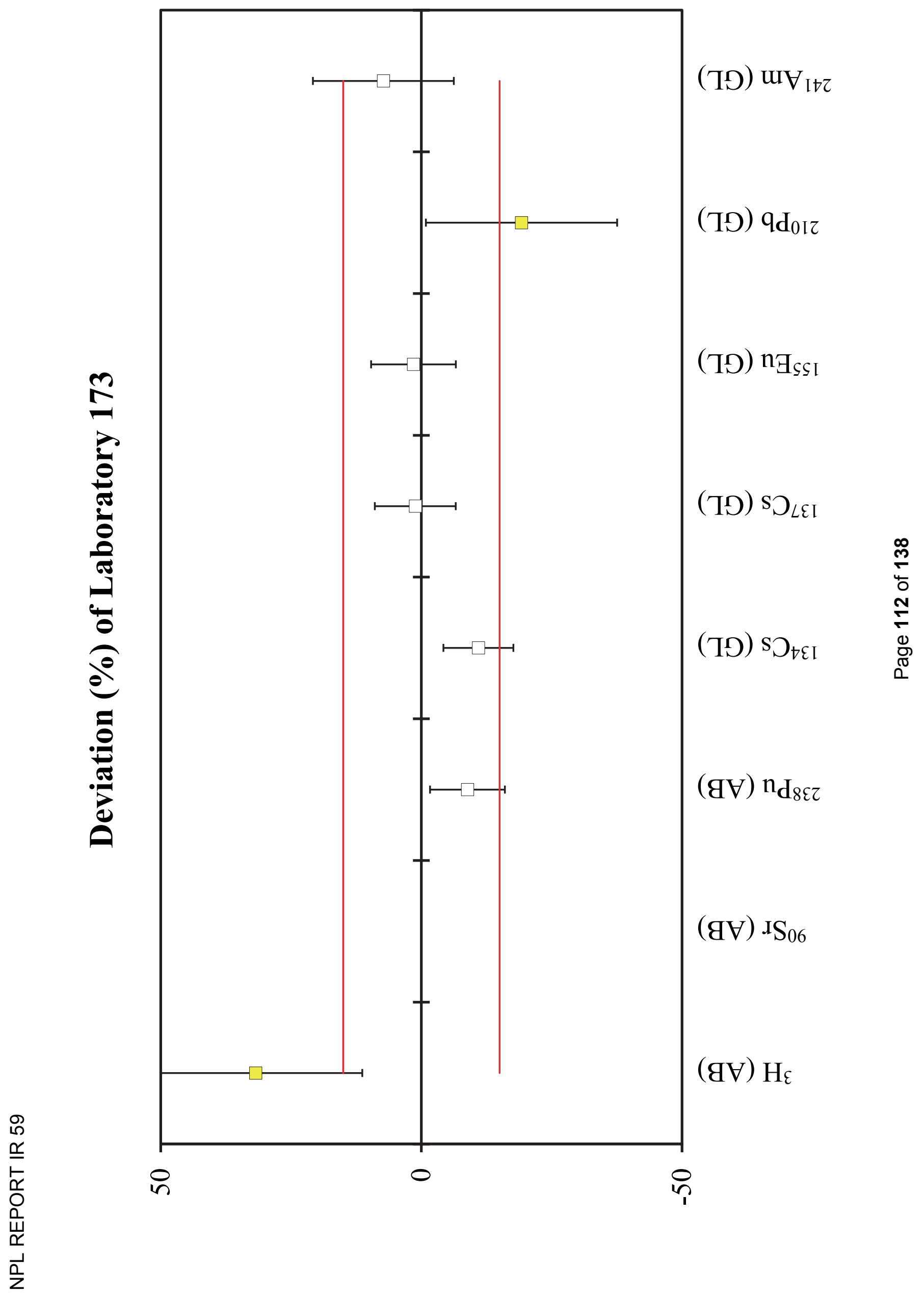




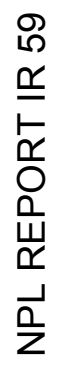

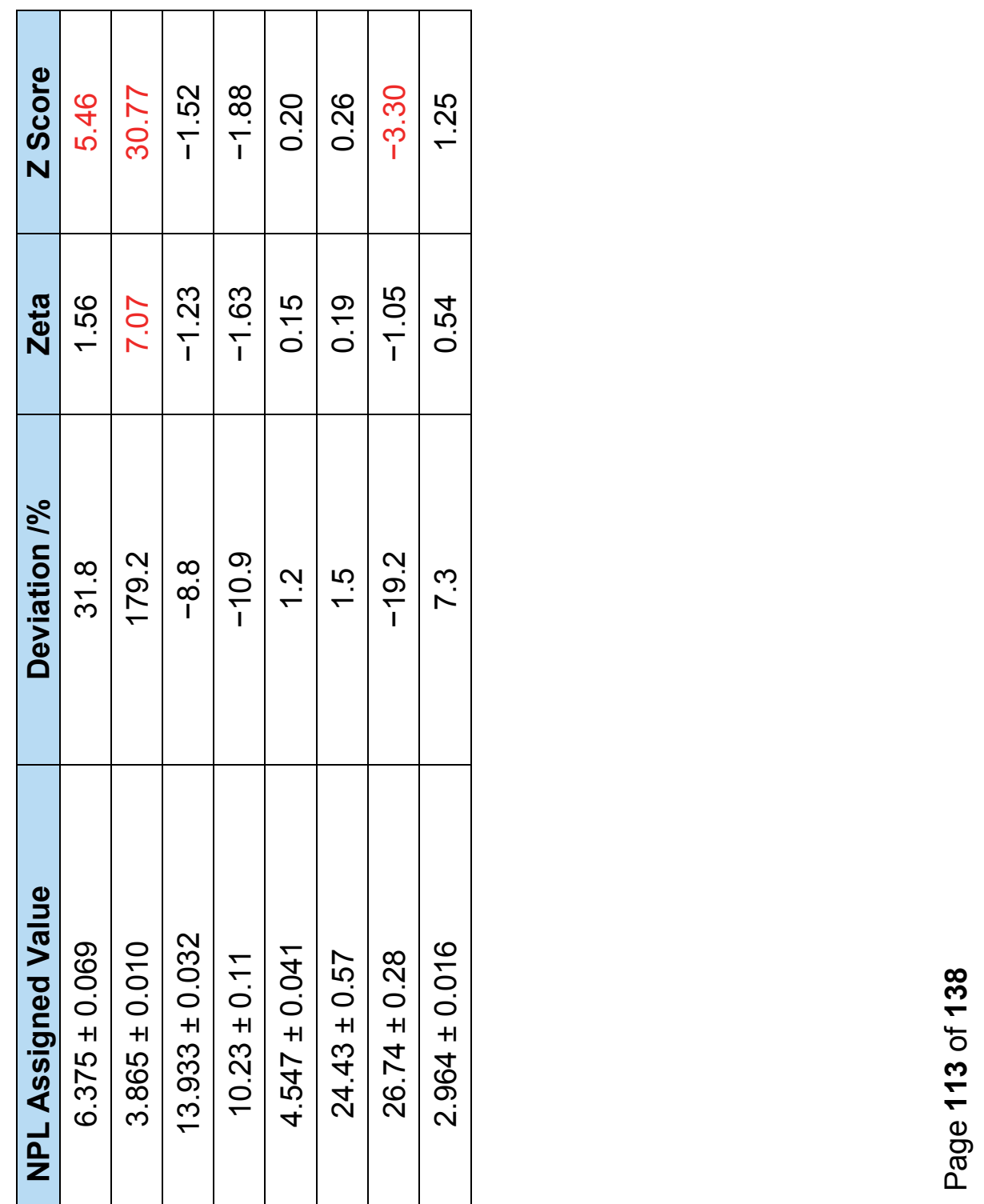

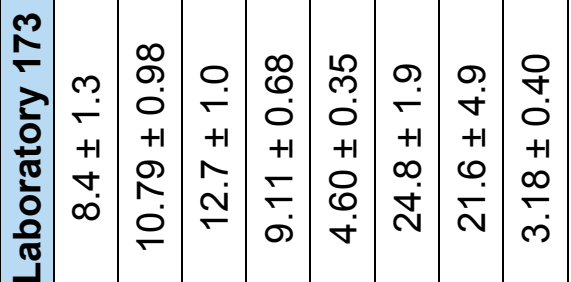

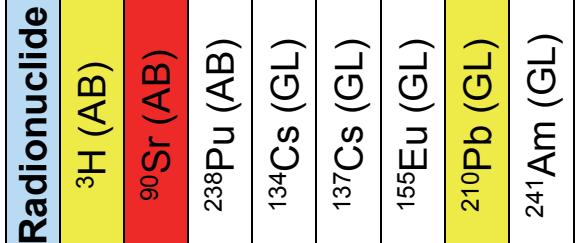




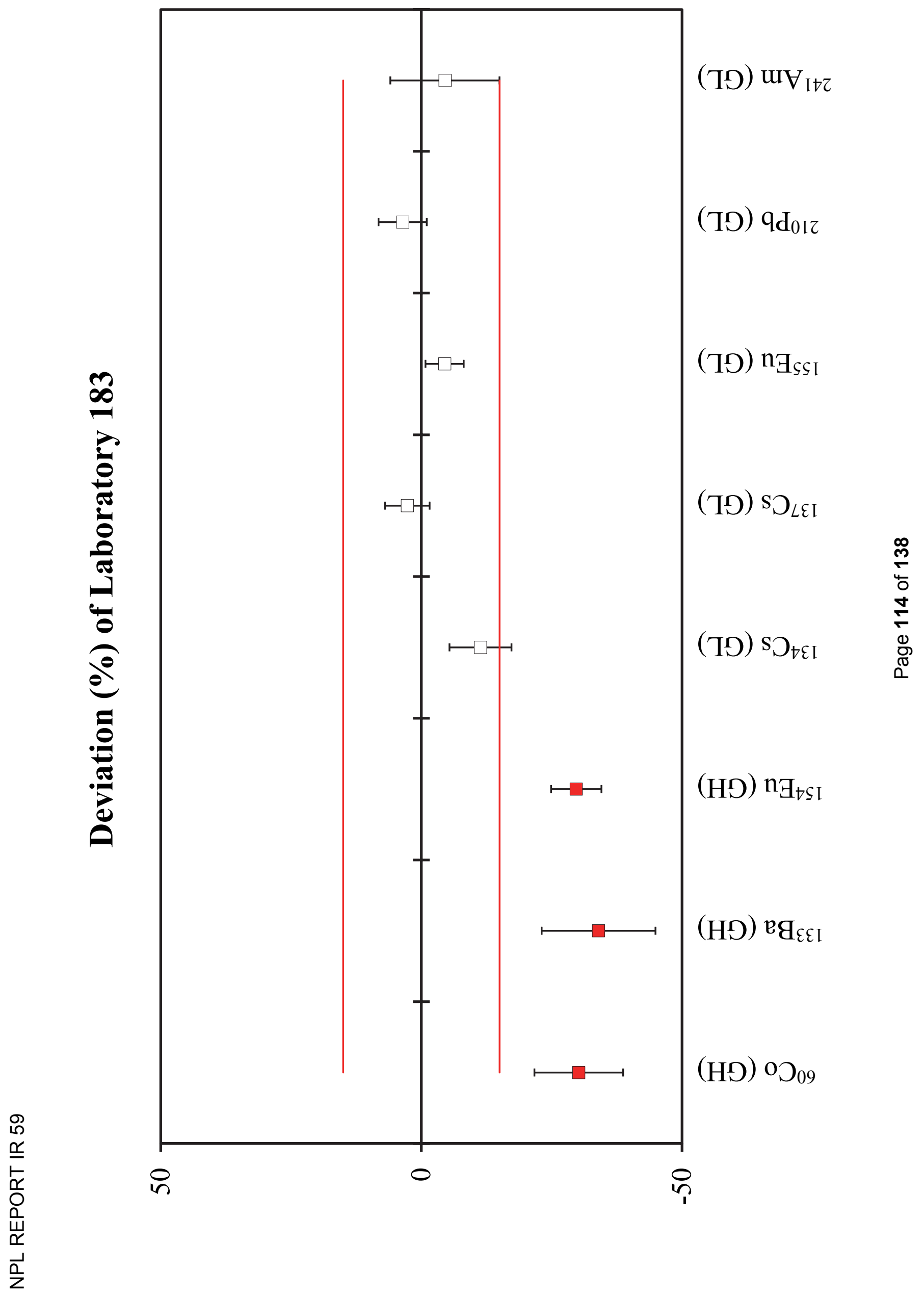




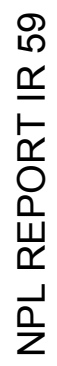

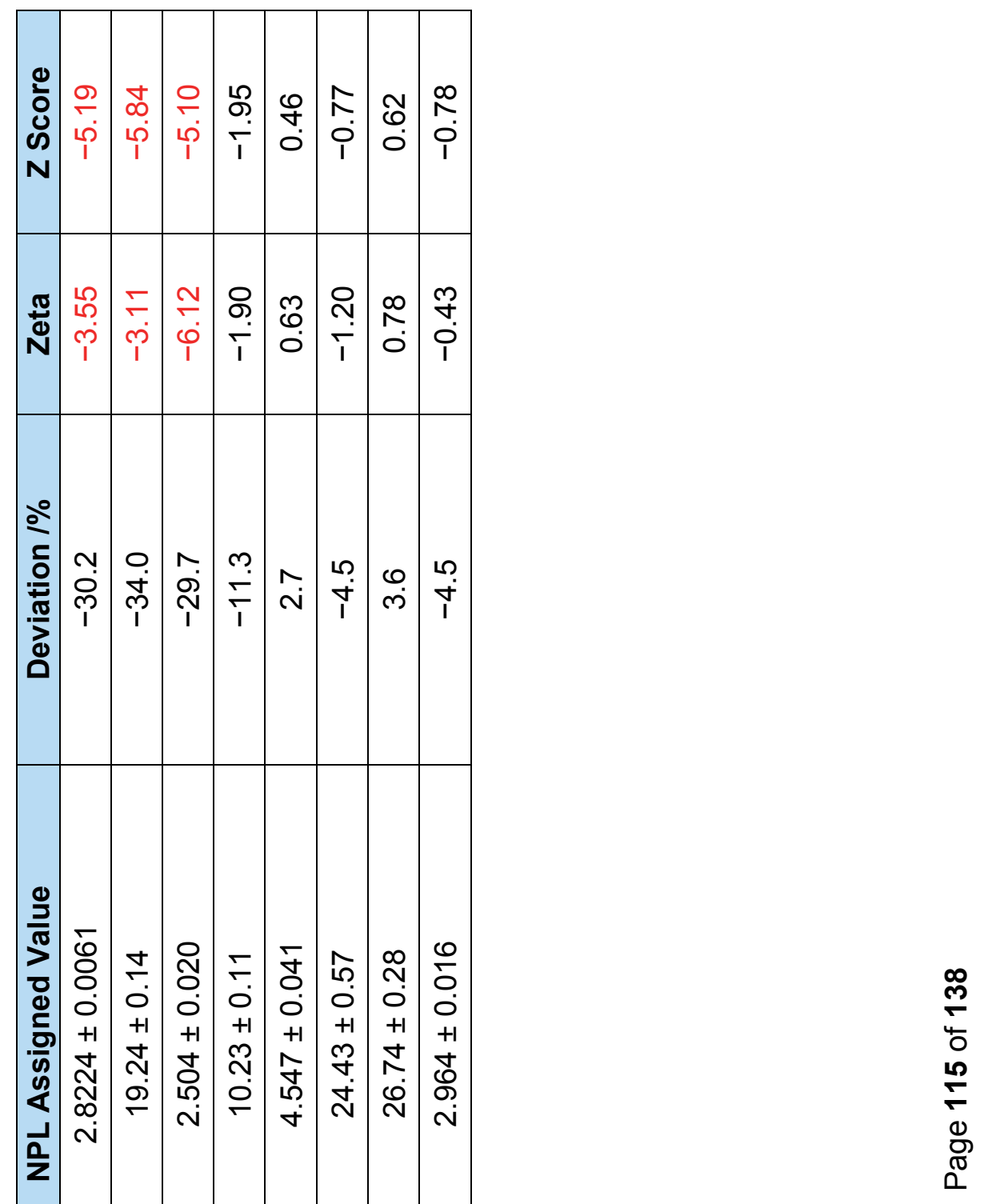

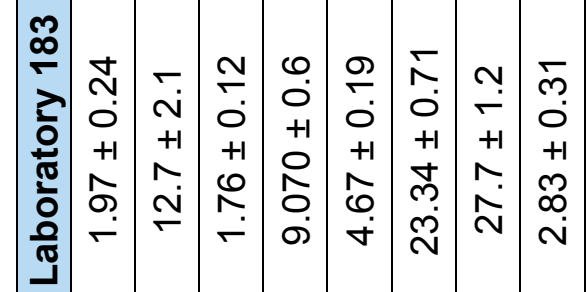

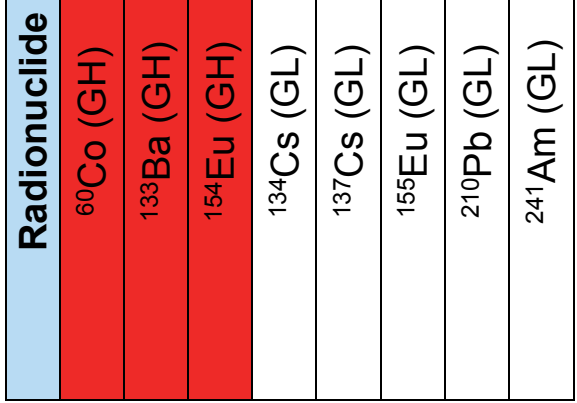




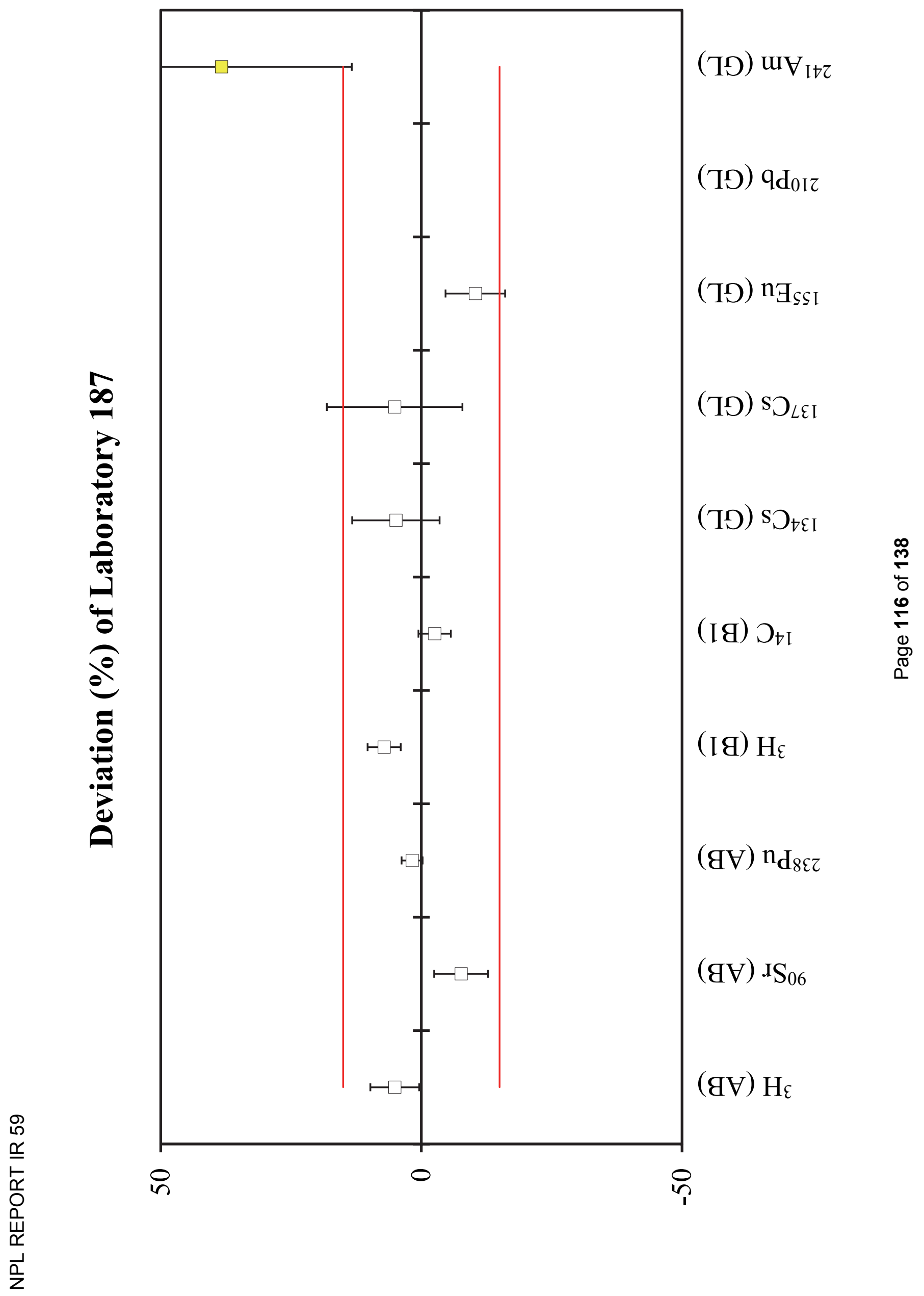




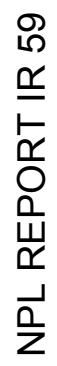

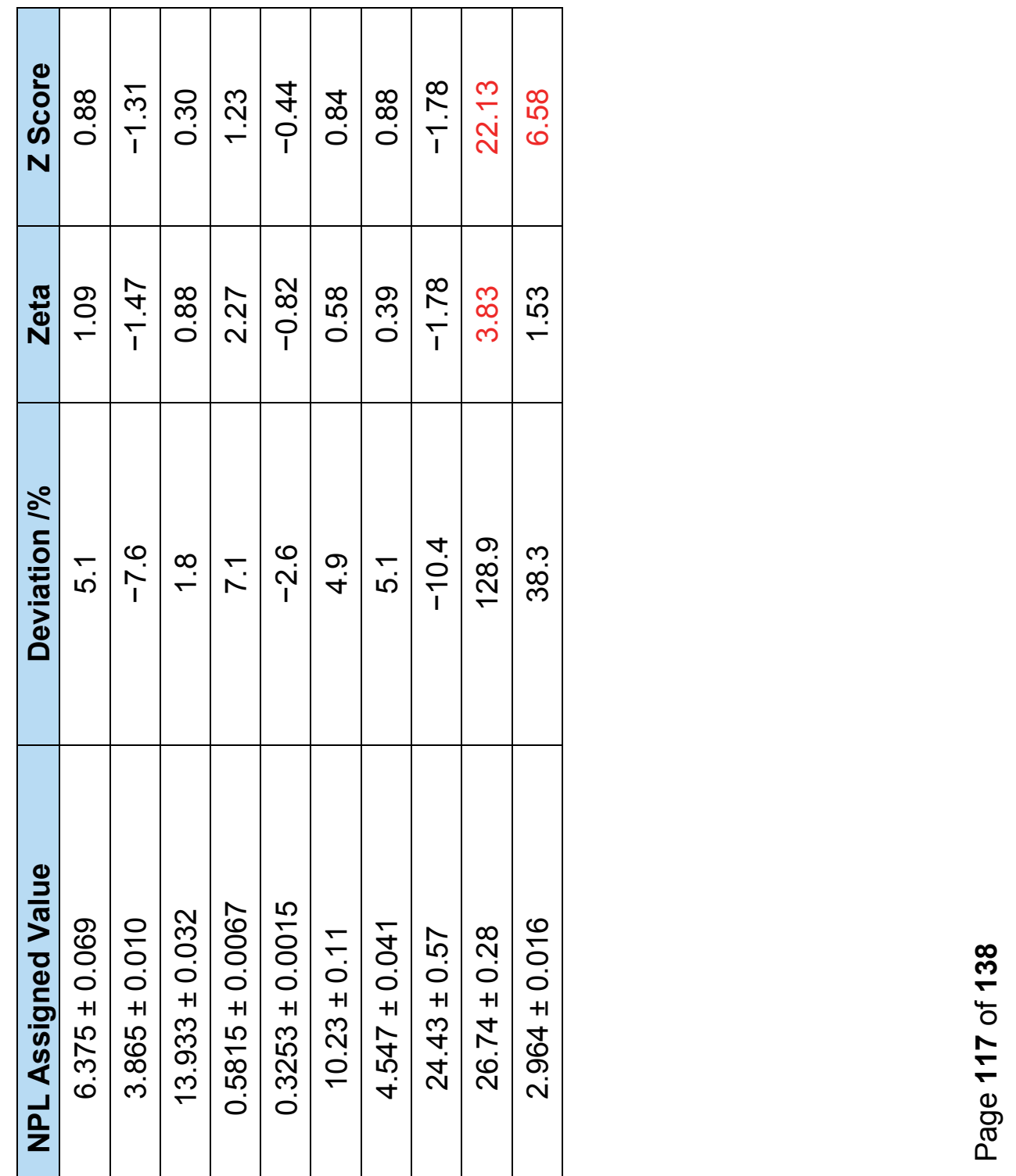

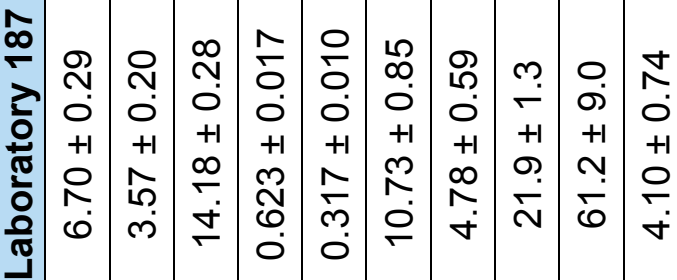




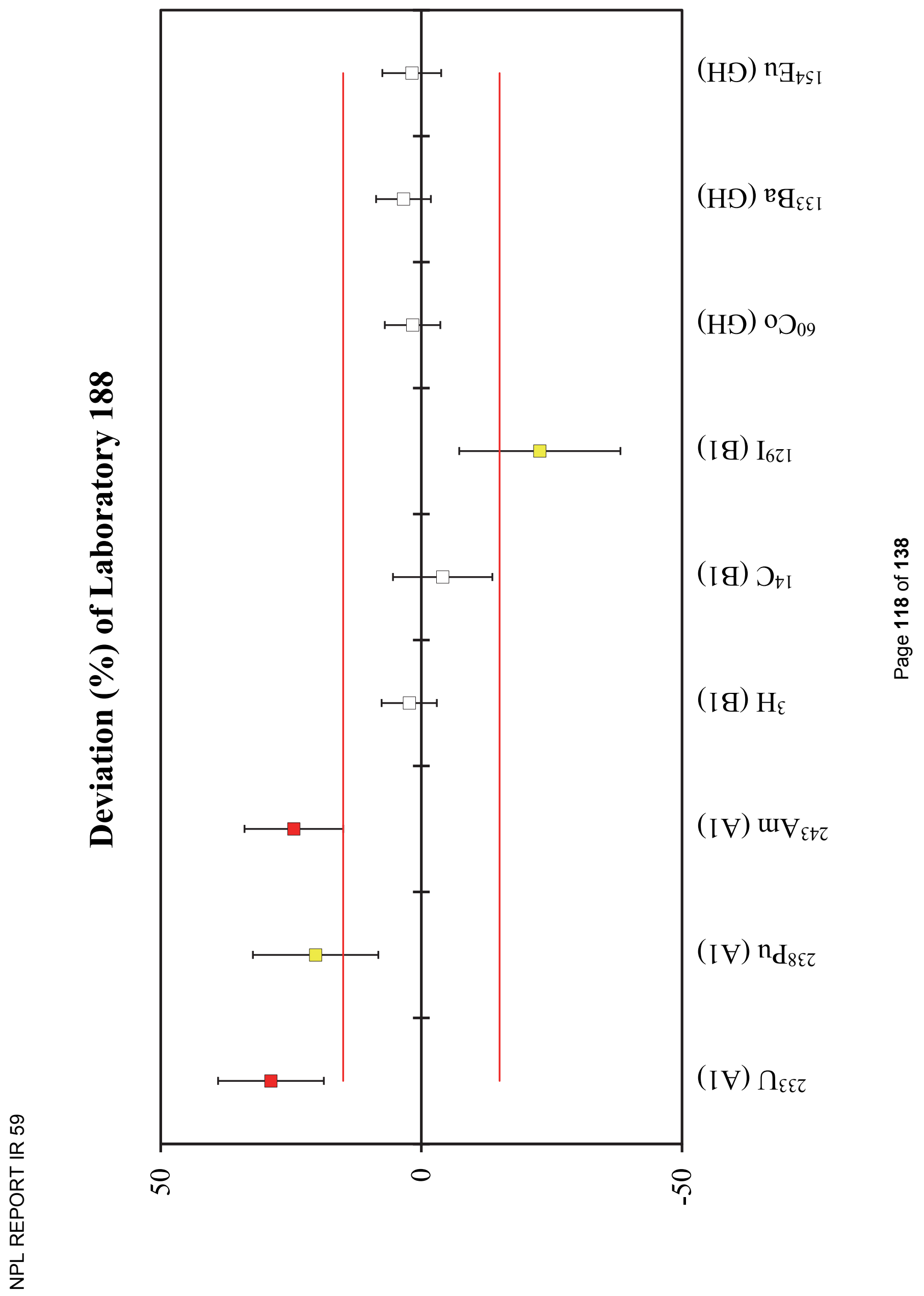




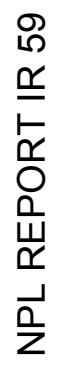

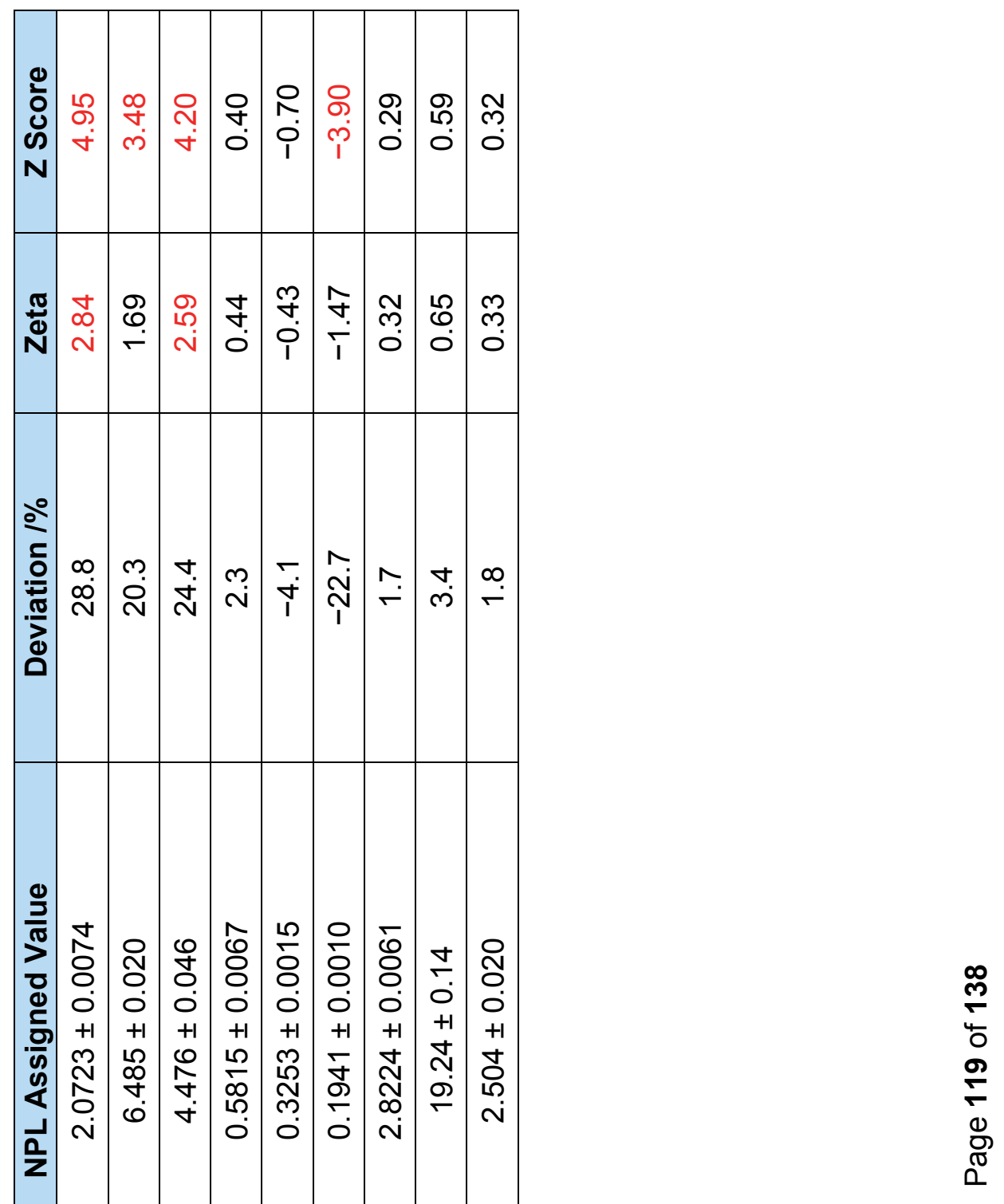

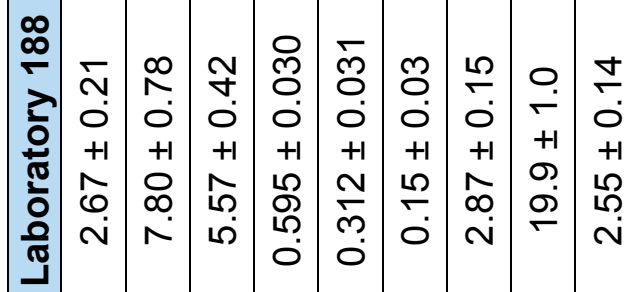

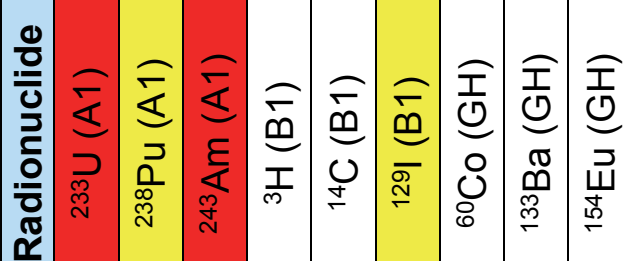




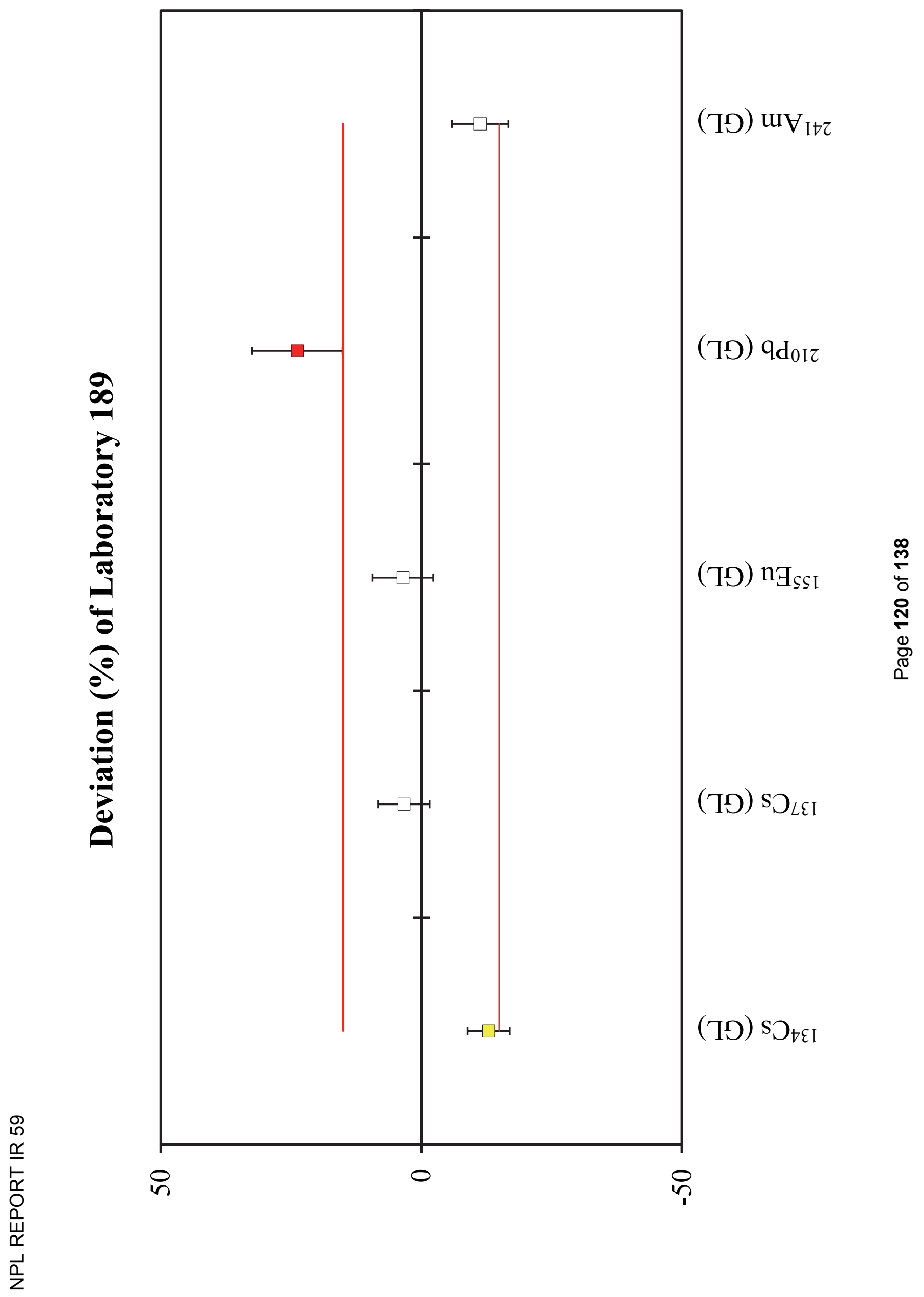




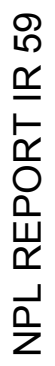

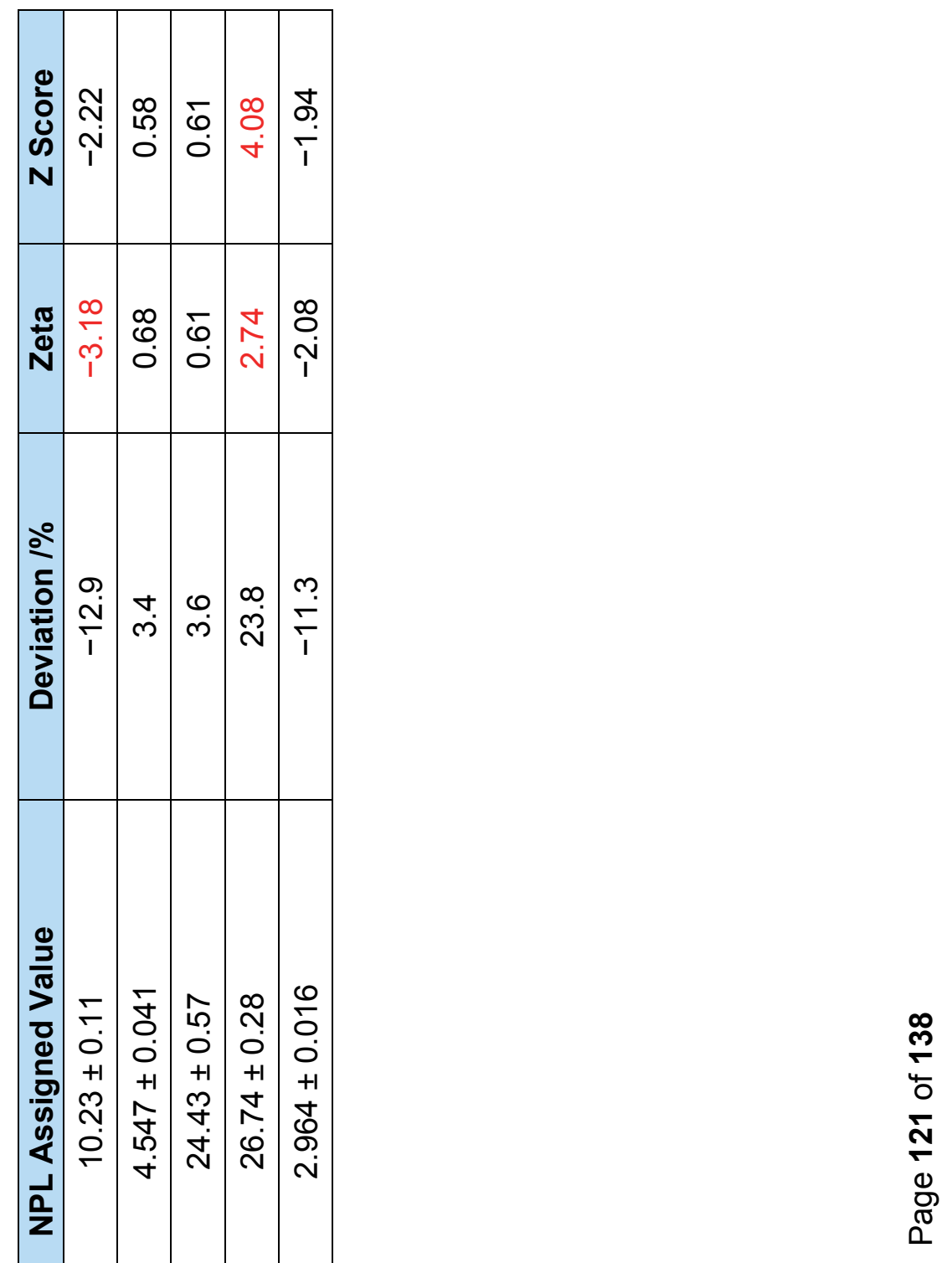

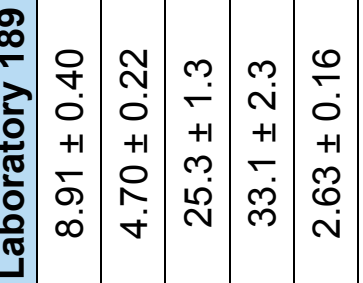

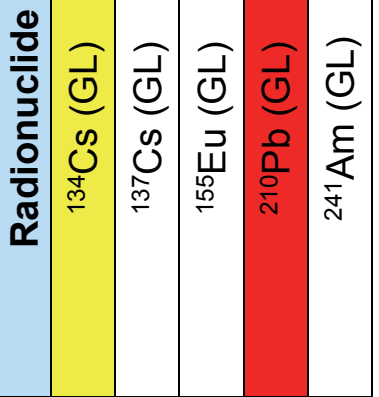




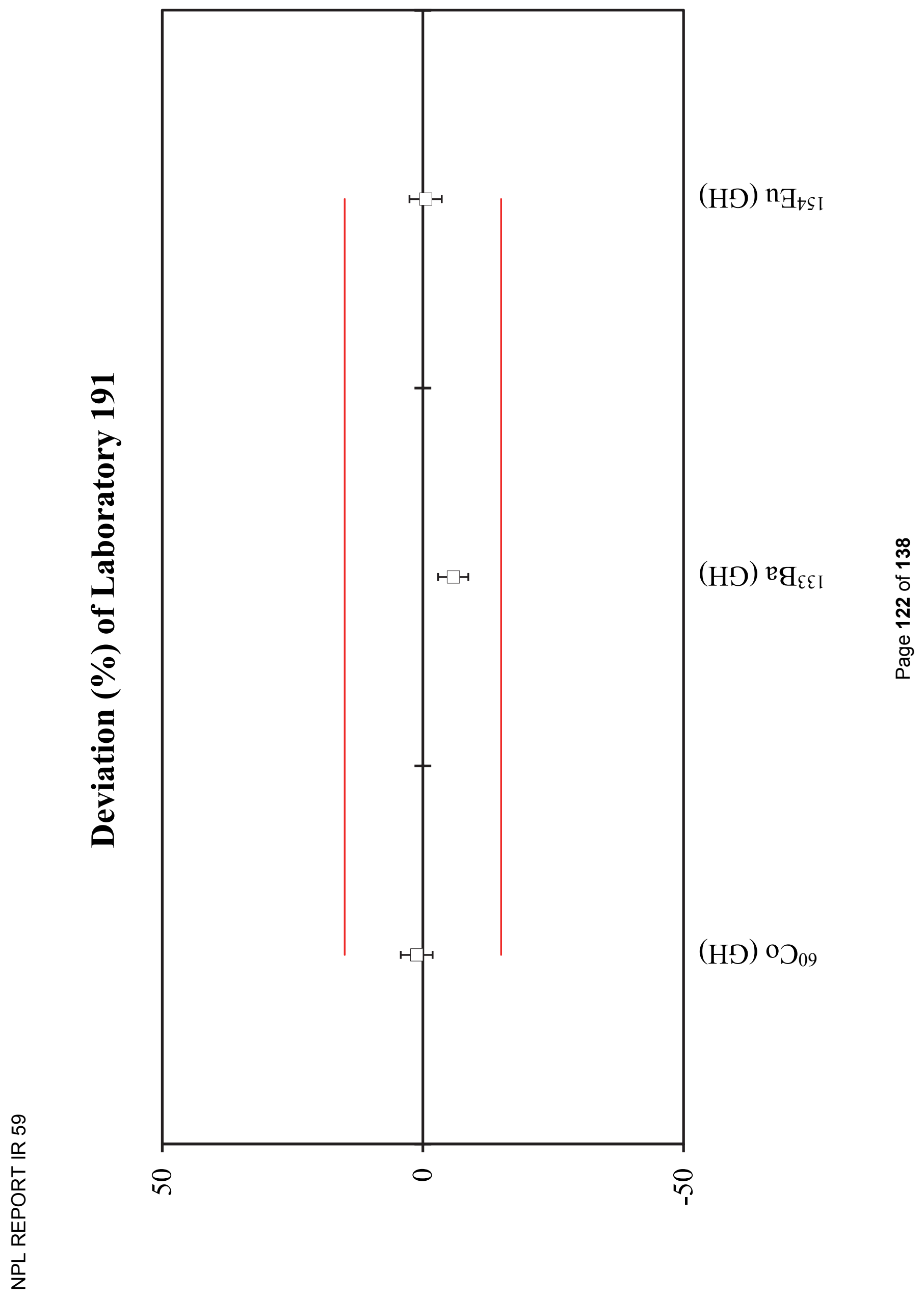




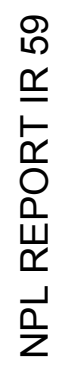

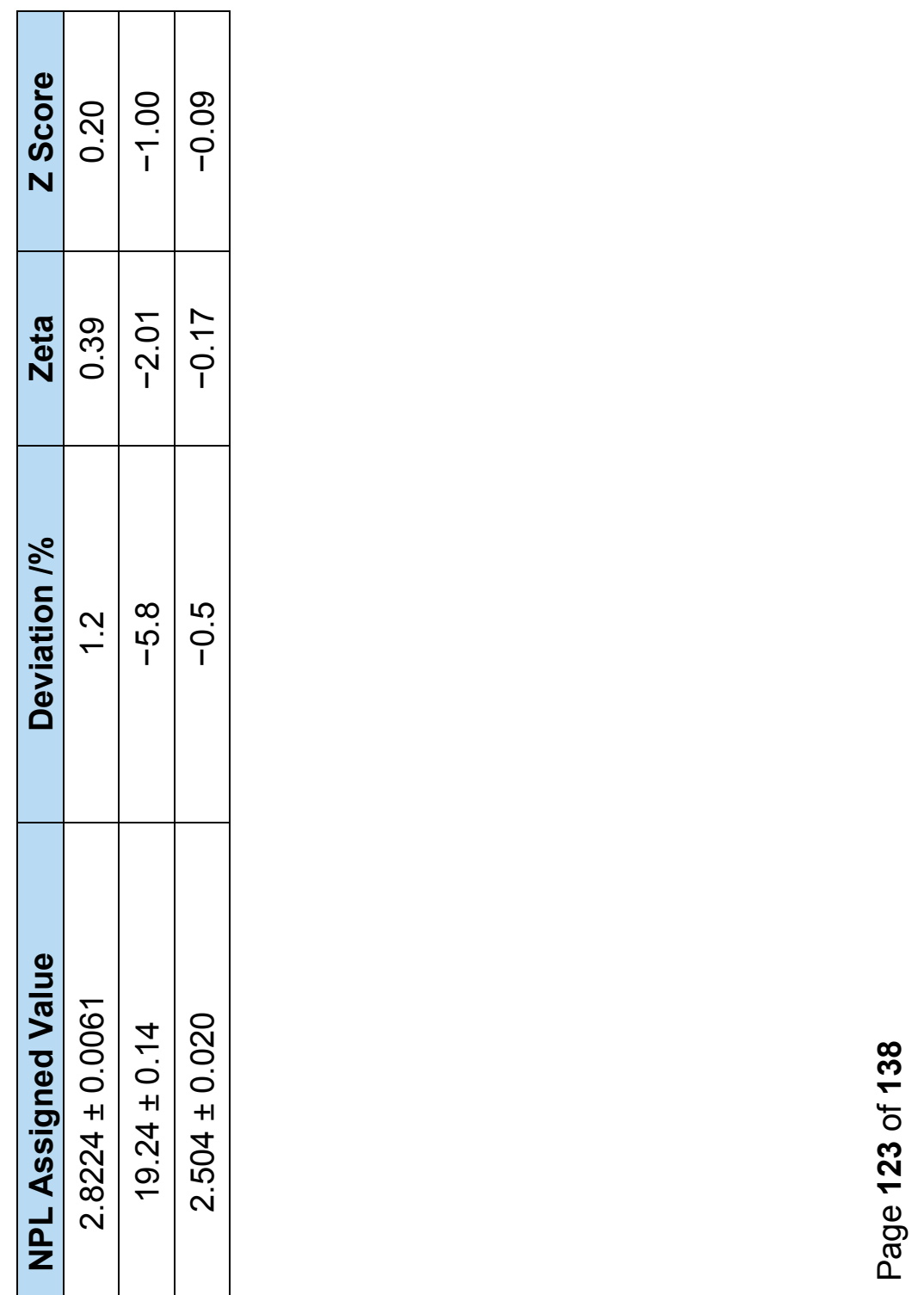

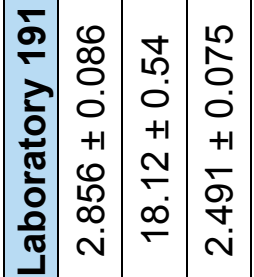

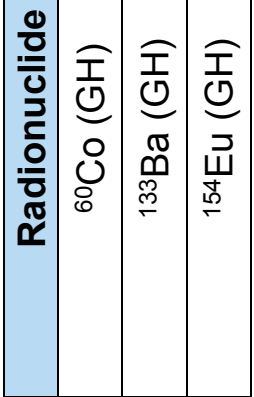




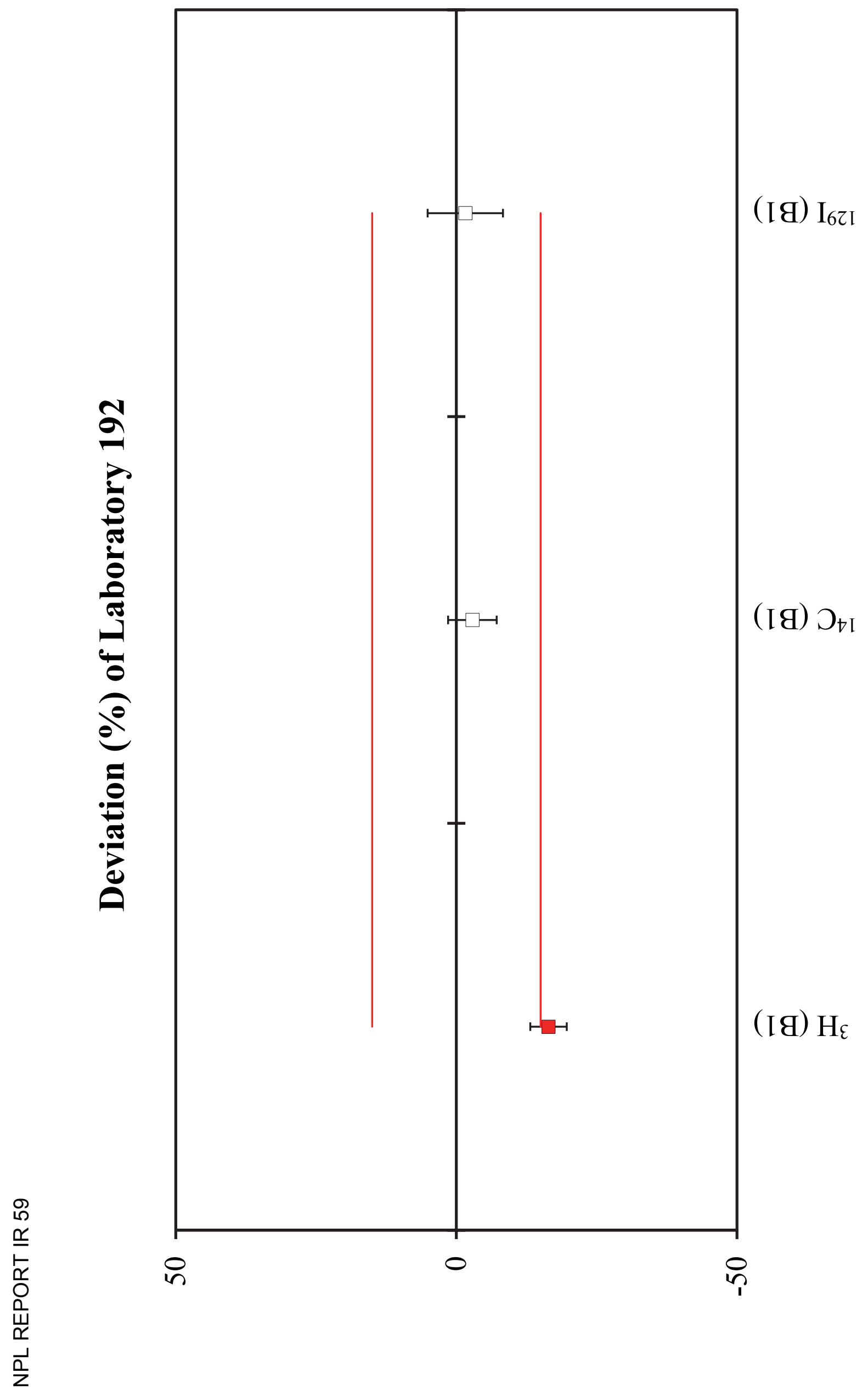

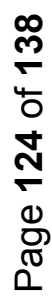




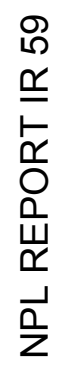

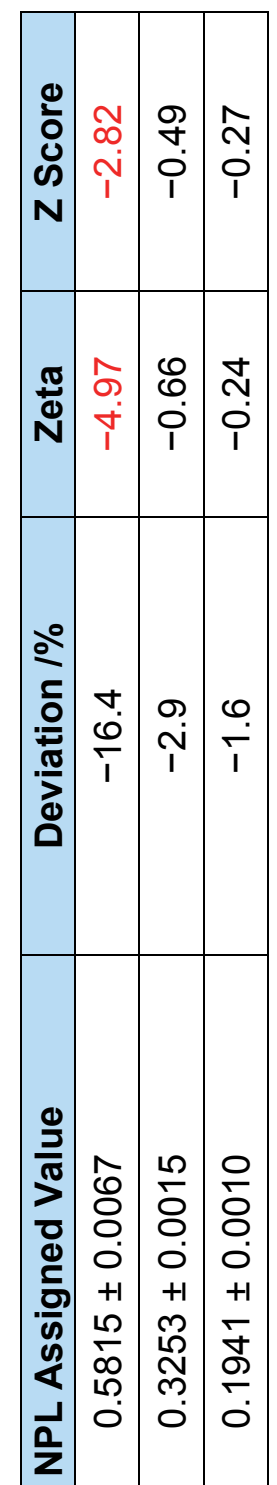

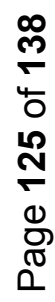

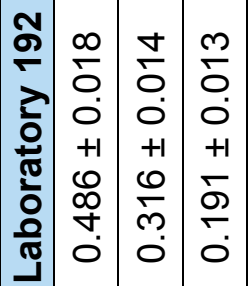

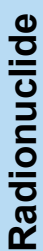

$\widehat{\widetilde{\Phi}} \widetilde{\tilde{g}}$

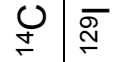




\section{DISCUSSION}

The following section discusses the reported results for the 2020 Environmental Proficiency Test Exercise. It should also be noted that in some cases participants did not report methods and/or techniques used. The information provided below, therefore, refers to a subset of participants (for each radionuclide) who did report such information. For NPL to provide performance-related feedback, it is encouraged that participants detail the methods and techniques used.

\subsection{Tritium in $\mathrm{AB}$}

The main difficulty in measuring the activity per unit mass of ${ }^{3} \mathrm{H}$ in the $A B$ sample type is the separation of the ${ }^{3} \mathrm{H}$ from the ${ }^{90} \mathrm{Sr}$ spectrum either by chemical separation or by setting regions of interest during measurement. Of the results submitted $73 \%$ were in agreement, $14 \%$ were questionable and $14 \%$ were discrepant. From the range of results provided there doesn't appear to be a systematic bias.

All of those who reported (17) the detection technique stated that they used LSC to measure ${ }^{3} \mathrm{H}$. Specific instruments reported as being used included a PerkinElmer

1220C QUANTULUS ${ }^{\mathrm{TM}}$ and a PerkinEImer Tri-Carb ${ }^{\circledR}$ 2910. The Tri-Carb ${ }^{\circledR}$ system has an internal source to evaluate the quenching correction. Quench is always present in aqueous samples and the degree of quench varies between samples. This means that for accurate results a quench correction should be carried out for each sample.

Distillation is commonly used to separate ${ }^{3} \mathrm{H}$ in aqueous samples from potential interferences. The majority of participants (9) that described a separation opted for distillation alone, with one participant describing pyrolysis followed by distillation. One participant used the distillation method described in ISO 9698:2019. Pyrolysis is used to turn a sample into a gaseous form. The gas is then passed through a catalytic reactor is oxidised to form tritiated water. The gas is trapped in tubes and a scintillation cocktail is added before measurement by LSC. Most of these devices have high recoveries but the yield should be monitored to quantify any losses. All participants that reported using a parallel standard agreed with the assigned value.

\subsection{Strontium-90 in $A B$}

The main difficulty in measuring the activity per unit mass of ${ }^{90} \mathrm{Sr}$ in $A B$ is the need for a radiochemical separation from the other radionuclides present in the sample, combined with the presence of ${ }^{89} \mathrm{Sr}$ which may also interfere. Several approaches can be adopted: decay and/or ingrowth counting, separation of ${ }^{90} \mathrm{Y}$ followed by Cerenkov and LSC counting and/or spectral deconvolution. Out of the reported results, $90 \%$ were in agreement, $5 \%$ were questionable and $5 \%$ were discrepant. These results are similar to the 2019 exercise where 26 results were submitted, of which $85 \%$ were in agreement, $8 \%$ were questionable and $8 \%$ were discrepant. From the range of results provided there doesn't appear to be a systematic bias.

Of the reporting laboratories 21 of the 18 provided techniques form. In most instances, the techniques form detailed the pre-treatment and separation techniques used before measurement. As with the 2019 exercise, the most common separation technique was extraction chromatography (7); of these laboratories, many stated using $\mathrm{Sr}$ resin from either TrisKem International or Eichrom Technologies.

One participant reported using the method described in ISO 11704:2018 for the analysis of the $A B$ sample type. This result has a deviation of $180 \%$ which may have been due to not applying a correction for the counting efficiency or an error in its application. It is important when measuring ${ }^{90} \mathrm{Sr}$ to either wait for the ${ }^{90} \mathrm{Y}$ to be in equilibrium or consider it during data analysis. 
Laboratories reported using detection techniques including Cherenkov counting (5), low-level alpha-beta counting (1), LSC (7) proportional counting (5). In some instances (3), like the method described above, the chemical yield was determined using ICP-MS measurement of stable $\mathrm{Sr}\left({ }^{88} \mathrm{Sr}\right)$ with other laboratories opting to add ${ }^{85} \mathrm{Sr}(3)$ as an internal tracer and measuring the recovery using gamma spectrometry. Parallel standards containing ${ }^{90} \mathrm{Sr}$ were also used (6).

The PPM of Cherenkov counting, LSC and proportional counting results were as follows:

Cherenkov Counting:

Liquid Scintillation Counting:

Proportional Counting:
$(3.73 \pm 0.10) \mathrm{Bq} \mathrm{g}^{-1}$

$(3.75 \pm 0.10) \mathrm{Bq} \mathrm{g}^{-1}$

(3.902 \pm 0.087$) \mathrm{Bq} \mathrm{g}^{-1}$

These results suggest there may be a significant difference between results obtained by proportional counting and the other detection techniques. As with the 2019 exercise, due to the size of the dataset and the varied methods leading up to the final analysis, it is difficult to confirm. The lower standard uncertainty on the PMM result for proportional counting confirms last year's finding that the uncertainties are not due to measurement precision but relate to a more consistent dataset. For last year's exercise, the standard uncertainty on the PMM for LSC was lower.

\subsection{Plutonium-238 in $A B$}

This year's exercise saw 18 results submitted for ${ }^{238} \mathrm{Pu}$ in $\mathrm{AB}$ sample type, down from 21 in the 2019 exercise. Of these results, $95 \%$ were in agreement and $5 \%$ were discrepant. The 2020 exercise saw a bias of $1.8 \%$ which may be compared to that of previous exercises: $-2.4 \%$ (2019) and 0.4\% (2017).

Radiochemical separation was performed using a variety of methods which included anion exchange, cation exchange and extraction chromatography. Many of the participants opted to directly separate the samples, but three opted for evaporation and precipitation before separation for additional sample clean up or quicker sample throughput. To trace the recovery isotopes of $\mathrm{Pu}$, including ${ }^{236} \mathrm{Pu}(2)$ and ${ }^{242} \mathrm{Pu}(9)$ were used.

All but one participant who reported their detection technique (14) measured ${ }^{238} \mathrm{Pu}$ using alpha spectrometry with one opting for LSC. The source preparation technique used for all the alpha spectrometry techniques was electrodeposition which provides good spectral resolution as compared to other techniques such as micro precipitation.

\subsection{Uranium-233 in A1}

This year's $\mathrm{A} 1 \mathrm{sample}$ contained a unique mix of isotopes, ${ }^{233} \mathrm{U},{ }^{238} \mathrm{Pu}$ and ${ }^{243} \mathrm{Am}$. Isotopic ratios of radioactive releases into the environment may be used to determine their origin. Uranium-233 may also be used in oceanography as an environmental tracer. Of the results submitted $64 \%$ were in agreement, $9 \%$ were questionable and $27 \%$ were discrepant. From the range of results provided there doesn't appear to be a systematic bias.

All participants (10) reported using alpha spectrometry to measure the ${ }^{233} \mathrm{U}$. The only reported tracer was ${ }^{232} \mathrm{U}$. Detection of ${ }^{23} \mathrm{U}$ was preceded by radiochemical separation followed by electrodeposition (5). A variety of separation techniques were used, including cation/anion exchange and extraction chromatography or a combination of the two.

One participant opted to boil the sample under reflux for approximately one hour to remove carbon dioxide. They then co-precipitated the radionuclides of interest with iron. This was followed by ion-exchange chromatography to separate the analytes which were individually electrodeposited onto stainless-steel discs. This result was in good agreement with the NPL value. 


\subsection{Plutonium-238 in A1}

This year's exercise saw 14 results for ${ }^{238} \mathrm{Pu}$ in $\mathrm{A} 1$. Of the results submitted $50 \%$ were in agreement, $21 \%$ were questionable and $29 \%$ were discrepant. Unlike the ${ }^{238} \mathrm{Pu}$ results for the $A B$ sample type, there was a large positive deviation away from the NPL value of $7.8 \%$.

Similar to the $A B$ sample type isotopes of $\mathrm{Pu}$, including ${ }^{236} \mathrm{Pu}(3)$ and ${ }^{242} \mathrm{Pu}(7)$, were used to trace the recovery following radiochemical separation. Radiochemical separation was performed using a variety of methods which included anion exchange, cation exchange and extraction chromatography. All participants who disclosed their detection technique use alpha spectrometry (11) to measure ${ }^{238} \mathrm{Pu}$. As with the $A B$ sample type, there was no use of mass spectrometry for the measurement of ${ }^{238} \mathrm{Pu}$. This is likely due to the interference of $238 \mathrm{U}$.

\subsection{Americium-243 in A1}

Americium-243 is an anthropogenic radionuclide that is rarely found in the environment and is often used as a yield tracer. Of the results submitted $50 \%$ were in agreement, $14 \%$ were questionable and $36 \%$ were discrepant.

Several laboratories (3) measured ${ }^{243} \mathrm{Am}$ directly by gamma spectrometry. It is assumed that the $74.7 \mathrm{keV}$ emission was used. One of these measurements was in agreement with the NPL assigned value whereas the others were discrepant; one above the assigned value and the other below.

Alpha spectrometry was the most popular measurement technique with ten laboratories opting to measure electrodeposited ${ }^{243} \mathrm{Am}$ post radiochemical separation. As with ${ }^{233} \mathrm{U}$ and ${ }^{238} \mathrm{Pu}$ both ion exchange and extraction chromatography was used. Laboratories that reported using a tracer (7) used ${ }^{241} \mathrm{Am}$ with one laboratory opting for ${ }^{244} \mathrm{Cm}$. The laboratory using ${ }^{244} \mathrm{Cm}$ as a tracer for an evaporation, ion exchange and electrodeposition-based method reported results in agreement with the assigned value.

The PPM of alpha spectrometry and gamma spectrometry results were as follows:
Alpha Spectrometry:
$(4.48 \pm 0.20) \mathrm{Bq} \mathrm{kg}^{-1}$
Gamma Spectrometry:
(4.57 \pm 0.50$) \mathrm{Bq} \mathrm{kg}^{-1}$

Laboratory 47.1 did not provide information on their applied techniques, but it is assumed their deviation is due to a reporting error as their reported value is one order of magnitude out.

\subsection{Tritium in $\mathrm{B} 1$}

This year's exercise saw 30 results reported, which is a similar number to the 2019 exercise which saw 32 . As with ${ }^{3} \mathrm{H}$ in the $\mathrm{AB}$ sample type, the main difficulty in measuring the activity per unit mass is the need to separate it from other beta-emitting radionuclides in the sample, i.e. ${ }^{14} \mathrm{C}$ and ${ }^{129}$. Of the results submitted $80 \%$ were in agreement, $10 \%$ were questionable and $10 \%$ were discrepant.

Of the participants who described their methodologies, 18 used distillation to isolate ${ }^{3} \mathrm{H}$ from interfering elements. The B1 sample matrix is an alkaline solution. Alkaline solutions often result in significant quenching and chemiluminescence. A few participants opted for a combustion-based technique where ${ }^{3} \mathrm{H}$ was trapped in nitric acid and ${ }^{14} \mathrm{C}$ onto Carbosorb. The only reported detection technique was LSC (26). Quantulus 1220c. Again, specific instruments used included a PerkinEImer 1220C QUANTULUS ${ }^{\mathrm{TM}}$ and a PerkinElmer TriCarb $^{\circledR} 2910$.

\subsection{Carbon-14 in B1}

The main difficulty in measuring the activity per unit mass of ${ }^{14} \mathrm{C}$ in $\mathrm{B} 1$ is the need for a radiochemical separation from ${ }^{3} \mathrm{H}$, and ${ }^{129}$. Of the results submitted $80 \%$ were in agreement, $10 \%$ were questionable and $10 \%$ were discrepant. There was also an overall 
improvement compared to the 2019 exercise where of the results $67 \%$ were in agreement, $19 \%$ were questionable and $14 \%$ were discrepant.

The difference between pyrolysis and combustion is that combustion is done is the presence of oxygen at atmospheric pressures. That being said, some pyrolysers have a dedicated oxygen line.

\section{9 lodine-129 in B1}

lodine-129 is a by-product of the nuclear industry. It is present in environmental samples at low activity concentrations but is considered important in radiological protection due to its long half-life. The main difficulty in measuring the activity per unit mass of ${ }^{129} \mathrm{I}$ in $\mathrm{B} 1$ is the need for a radiochemical separation from ${ }^{3} \mathrm{H}$, and ${ }^{14} \mathrm{C}$. Of the results submitted $65 \%$ were in agreement, $29 \%$ were questionable and $6 \%$ were discrepant.

Detection of ${ }^{129}$ in the $\mathrm{B} 1$ sample type was determined using a variety of techniques including, LSC (6), gamma spectrometry (5) and mass spectrometry (1) with one laboratory opting for a combined detection using LSC (TDCR) and gamma spectrometry).

The PPM of gamma spectrometry and LSC results were as follows:

Gamma Spectrometry:

Liquid Scintillation counting:
$(0.192 \pm 0.012) \mathrm{Bq} \mathrm{g}^{-1}$

$(0.1994 \pm 0.0076) \mathrm{Bq} \mathrm{g}^{-1}$

These results suggest there is no significant difference between results obtained using each detection technique. The lower precision on the PMM of the gamma spectrometry technique is considered to be indicative of the challenges faced when measuring ${ }^{129} \mathrm{I}$ due to its low energy gamma-ray emission at $39.6 \mathrm{keV}$.

One participant opted to detect ${ }^{129}$ I by ICP-MS; submitting a result in agreement with the assigned value. Stable I $\left({ }^{127} \mathrm{I}\right)$ is present in environmental samples and often causes measurement issues. Other challenges impacting the measurement of ${ }^{129}$ by ICP-MS include the potential isobaric interference from ${ }^{129} \mathrm{Xe}$ which is an impurity in the argon plasma gas, this may be corrected for using a baseline subtraction if no inline separation mechanism exists. The energy to ionise I is also high $(10 \mathrm{eV})$ which means that instrument sensitivity is relatively low compared to other elements. When measuring by mass spectrometry it is important to correct for ion suppression, either through the use of matrixmatched standards or use of an internal standard.

\subsection{Sample Types GH and GL}

A similar number of datasets were provided for the 2020 as for the 2019 exercise. As with previous exercises, the samples included radionuclides with a wide range of emission energies.

The GL sample for this year's exercise contained challengingly low-levels of ${ }^{241} \mathrm{Am}$ and ${ }^{210} \mathrm{~Pb}$. The amount of ${ }^{210} \mathrm{~Pb}$ in the $\mathrm{GL}$ was increased from the previous exercise to allow participants' without low background detection facilities to report.

The majority of questionable and discrepant results were for ${ }^{210} \mathrm{~Pb}$ and ${ }^{241} \mathrm{Am} ; 80 \%$ of the discrepant results were for ${ }^{210} \mathrm{~Pb}$ and $78 \%$ of the questionable results were for ${ }^{210} \mathrm{~Pb}$ and ${ }^{241} \mathrm{Am}$ combined. All but one of the reported measurements for the $\mathrm{GH}$ and $\mathrm{GL}$ sample types were carried out by high-resolution gamma spectrometry. The one alternative measurement technique used was for ${ }^{241} \mathrm{Am}$. This measurement for ${ }^{241} \mathrm{Am}$ was by alpha spectrometry using ${ }^{243} \mathrm{Am}$ as a yield tracer; the reported result was in agreement with the assigned value.

This low-level of performance may be attributed in part to the availability of suitable commercially available mixed calibration standards. Many mixed standards do not include ${ }^{210} \mathrm{~Pb}(46.5 \mathrm{keV})$, and only go as low as $60 \mathrm{keV}$. For laboratories routinely measuring isotopes with emissions below $60 \mathrm{keV}$, it is advised that the calibration points of the fullenergy detection efficiency calibration extend beyond the relevant emission energies. For emission energies beyond the highest calibration point for the full-energy detection 
efficiency, the effects are less significant due to the linearity in this region of the calibration curve. When measuring gamma-emitting radionuclides with low emission energies such a ${ }^{210} \mathrm{~Pb}$ and ${ }^{241} \mathrm{Am}(59.5 \mathrm{keV})$ it is also important to determine and apply self-attenuation correction factors relating to sample density. The lower the emission energy, the larger the self-attenuation correction factor. Lead-210 is also a difficult radionuclide to measure due to the presence of ${ }^{210} \mathrm{~Pb}$ in background measurements.

For the cases of ${ }^{60} \mathrm{Co},{ }^{133} \mathrm{Ba},{ }^{154} \mathrm{Eu},{ }^{134} \mathrm{Cs}$ and ${ }^{155} \mathrm{Eu}$ true coincidence summing of emissions needs to be considered where the source to detector geometry is close. This can be of a particular challenge for those radionuclides with low-energy gamma-ray emissions in coincidence with other gamma rays, e.g. ${ }^{133} \mathrm{Ba}$, where the total efficiency modelled at the lower energy can be difficult to determine accurately. In general, the results for all of these radionuclides showed agreement with the Assigned Value with one exception.

As observed in Table 8, there is a discrepancy between the Assigned Value and the PMM for the ${ }^{134} \mathrm{Cs}$ in the GL sample. There is a low bias of the PMM with a deviation of $-3.7 \%$ (zeta-score $=2.71$, critical value $=2.58$ ) from the Assigned Value. Of the 27 reported results, $22(81.5 \%)$ were less than the Assigned Value, though only two of these were questionable due to the uncertainties quoted and there were no discrepancies. It may be considered that whilst the low bias of the participant results observed could be due to a number of factors, e.g. low counting statistics, continuum subtraction, true summing corrections, that in general the individually reported results have acceptable uncertainty budgets accounting for these components. However, whilst the average standard uncertainty quoted by participants is $7.8 \%$ the standard uncertainty of the PMM is $0.89 \%$. The PMM technique considers all results to be independent and does not consider correlations between them, which is important where the same technique is being used. If these correlations are not accounted for then the standard uncertainty of the PMM could be underestimated where the dataset tends towards the weighted mean of all the results $\left(\alpha_{c s-134}=1.88\right)$. This may explain the resulting discrepancy of the PMM and the Assigned Value.10.11 Measurement uncertainties

This exercise includes results for multiple measurands using a variety of methodologies. The measurement uncertainties arising for each method will include different components.

Participants are encouraged to review their uncertainty budgets to ensure that they are comprehensive and provide a reasonable estimation of the overall uncertainty.

There are instances where results are marked as questionable due to failing the relative uncertainty test. In these instances, it is suggested that participants' review their uncertainty budgets. Following review, if the uncertainty budget is deemed to be comprehensive it may be that the $R_{\mathrm{L}}$ test failed due to differences in the applied method as compared with other participating laboratories.

An example of an uncertainty budget for a direct gamma spectrometry measurement of an environmental sample is given below in Table 9. 
Table 9 Sources of uncertainty for a measurement of an environmental sample by gamma spectrometry (IAEA-TECDOC-1401, 2004).

\begin{tabular}{|c|c|c|c|}
\hline Uncertainty Source & Symbol Used & $\begin{array}{c}\text { Typical Uncertainty } \\
\text { Range (\%) }\end{array}$ & $\begin{array}{c}\text { Typical Uncertainty } \\
\text { Value (\%) }\end{array}$ \\
\hline Counting & $N$ & $0.1-20$ & 5 \\
\hline Emission probability & $V$ & $0.1-11$ & $<2$ \\
\hline $\begin{array}{c}\text { Attenuation } \\
\text { correction }\end{array}$ & $K_{3}$ & $0.1-5$ & $<3$ \\
\hline $\begin{array}{c}\text { Coincidence } \\
\text { correction }\end{array}$ & $K_{5}$ & $1-15$ & $<0.2$ \\
\hline Half-life & $T_{1 / 2}$ & $0.01-1$ & 2 \\
\hline $\begin{array}{c}\text { Detector efficiency } \\
\text { Radiochemical } \\
\text { procedures }\end{array}$ & $\varepsilon$ & $1-5$ & 3 \\
\hline Sample weight & $M$ & $1-10$ & $<0.5$ \\
\hline
\end{tabular}




\section{REFERENCES}

van Es, E. et al., 2021. Environmental Radioactivity Proficiency Test Exercise 2019. NPL Report IR 56 Issue 2. Available at https://www.npl.co.uk/products-services/radioactivity/environmental-pte

Harms, A. and Gilligan, C., 2011. Environmental Radioactivity Proficiency Test Exercise 2010. NPL Report IR 26. Available at https://www.npl.co.uk/products-services/radioactivity/environmental-pte

Pommé, S., 2012. Determination of a reference value, associated standard uncertainty and degrees of equivalence. European Commission Scientific and Technical Research series. ISSN 1831-9424 (online), ISBN 978-92-79-25104-7 (pdf).

Pommé, S., 2015. Determination of a reference value and its uncertainty through a powermoderated mean. Metrologia, 52, S200

BS ISO 13528:2015. Statistical methods for use in proficiency testing by inter-laboratory comparisons.

ISO 9698:2019. Water quality — Tritium — Test method using liquid scintillation counting

ISO 10704:2019. Water quality — Gross alpha and gross beta activity — Test method using thin source deposit.

ISO 11704:2018. Water quality - Gross alpha and gross beta activity - Test method using liquid scintillation counting.

IAEA-TECDOC-1401. 2004. Quantifying uncertainty in nuclear analytical measurements. ISSN 1011-4289 (online), ISBN 92-0-108404-8 (pdf).

\section{ACKNOWLEDGEMENTS}

The authors wish to thank the participating organisations for the time and effort they have put into analysing the samples. They also thank colleagues Daniel Ainsworth and Steph Perry for handling despatch of samples, Anu Bhaisare for her help in preparing the samples, Arzu Arinc for her role as Quality Manager, and Jane Taylor for leading communications with participants. The authors also wish to thank Andy Pearce for his technical contribution and Ben Russell for assisting with the final review and approval of the report. 


\section{APPENDIX I GROSS MEASUREMENT RESULTS SUMMARY}

A decision was made to not use the PMM as the Assigned Value for the gross measurements due to the limited number of results submitted, the spread of those results and the variation in measurement techniques used. As stated in the 2019 report Gross alpha and beta measurements are used to screen for radioactivity in samples (ISO 10704:2019; ISO 11704:2018) and are not suitable for absolute determination of the activity per unit mass of all alpha and beta-emitting radionuclides. Gross alpha and beta measurements are employed to ensure reference levels of specific alpha- and beta-emitting particles have not been exceeded. Gross alpha and gross beta analysis are not expected to be as accurate nor as precise as specific radionuclide analysis after radiochemical separations.

The values provided in the following tables are the PMM of the submitted results and are not traceable to national standards of radioactivity. The PMM of the gross measurements is provided as an indicator and has not been used for performance assessment. It is for this reason results for gross measurements do not appear in the main body of the report.

A 1 Gross radionuclide measurements summary

\begin{tabular}{|c|c|}
\hline Measurement & PMM \\
\hline Gross beta (AB) & $7.23 \pm 0.46 \mathrm{~Bq} \mathrm{~g}^{-1}$ \\
\hline Gross alpha (A1) & $14.9 \pm 1.2 \mathrm{~Bq} \mathrm{~kg}^{-1}$ \\
\hline Gross beta (B1) & $0.58 \pm 0.45 \mathrm{~Bq} \mathrm{~g}^{-1}$ \\
\hline
\end{tabular}




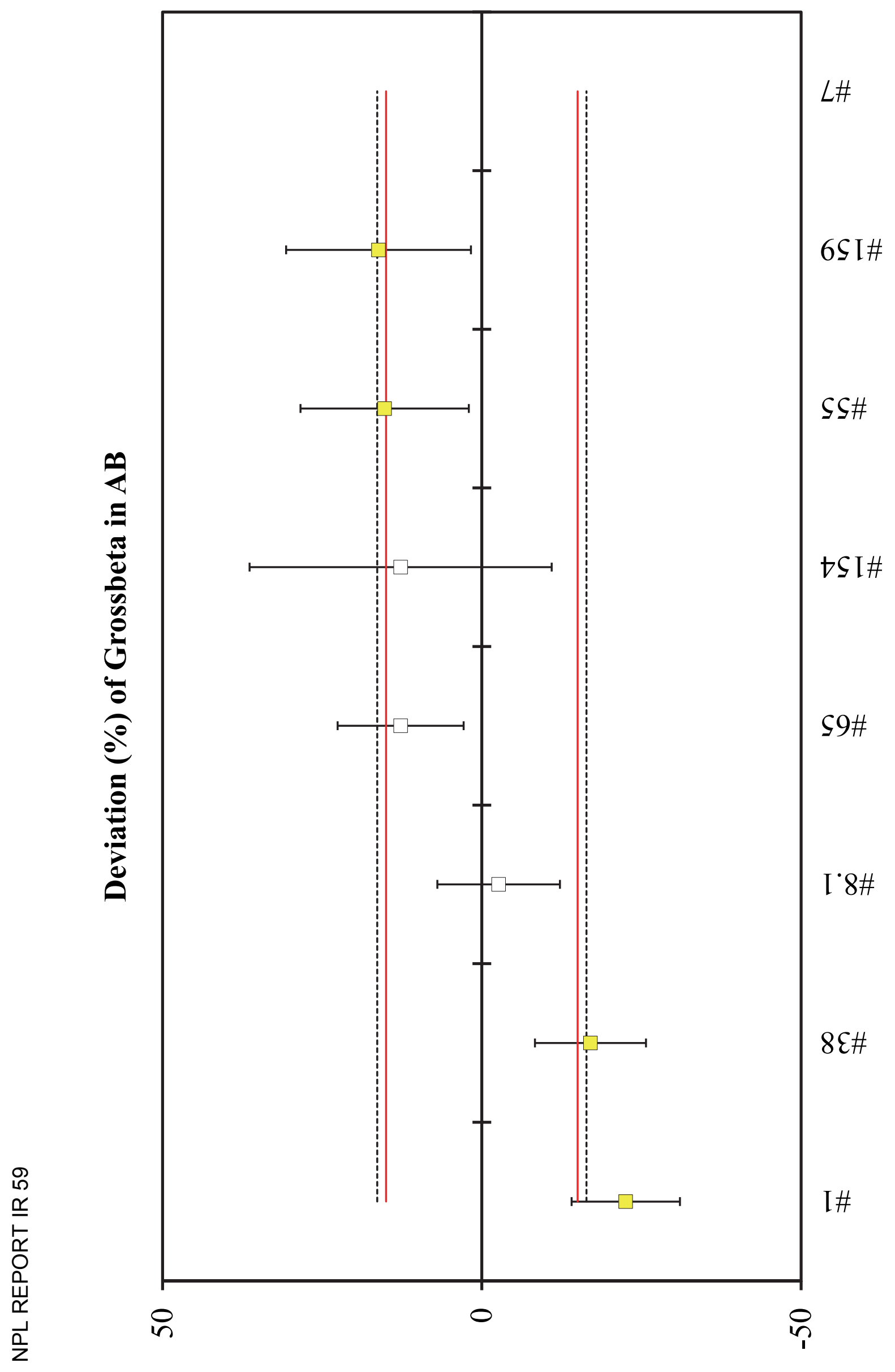




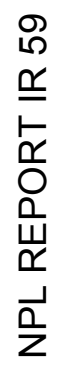

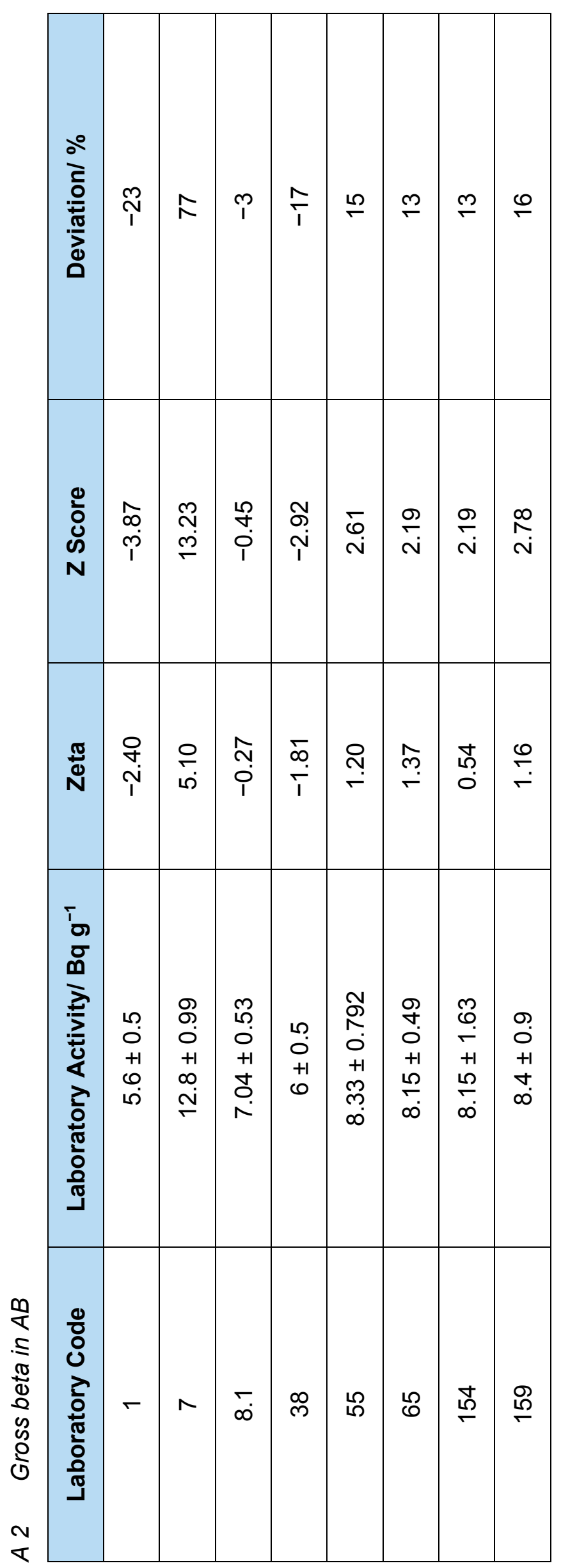

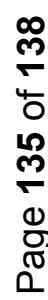




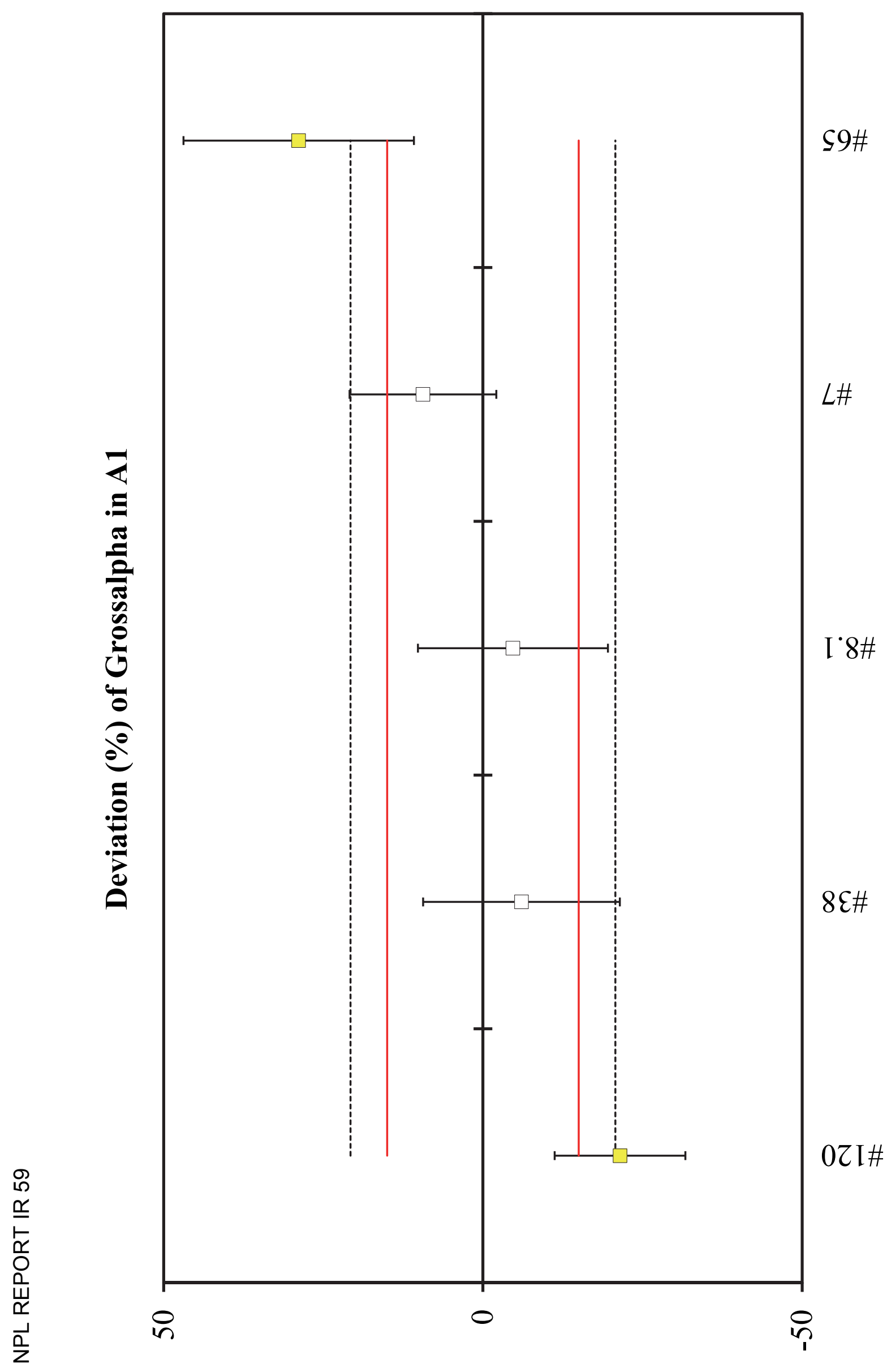

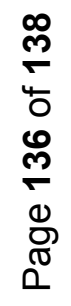




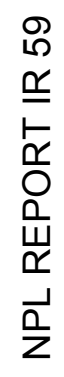

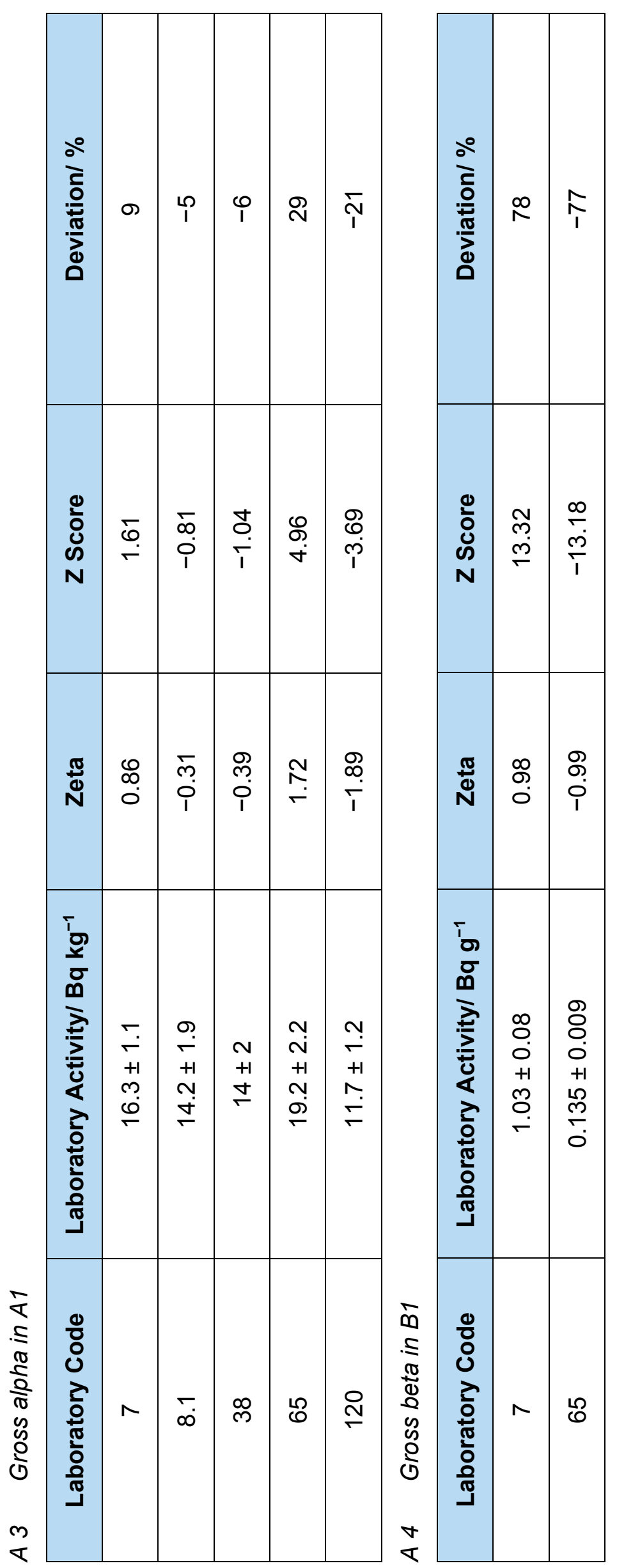

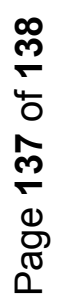




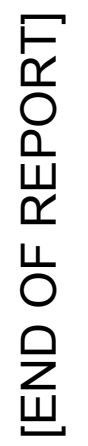

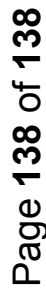

요

$\underline{\underline{ }}$

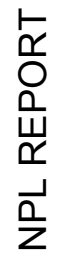

